\title{
SILICA-REINFORCED NATURAL RUBBER
}

\section{USE OF NATURAL RUBBER GRAFTED WITH CHEMICAL FUNCTIONALITIES AS COMPATIBILIZER}

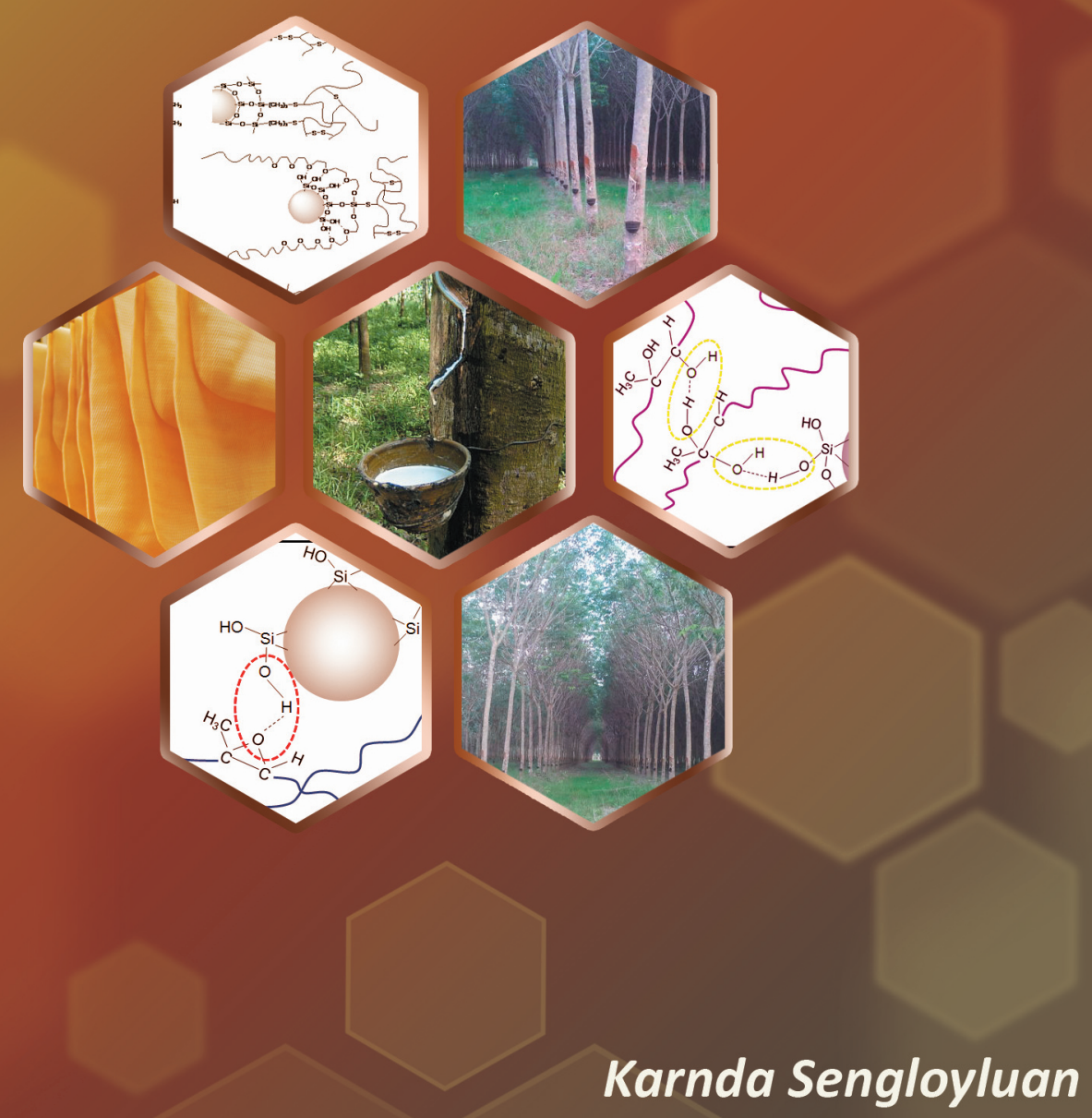




\section{SILICA-REINFORCED NATURAL RUBBER}

USE OF NATURAL RUBBER GRAFTED WITH CHEMICAL

FUNCTIONALITIES AS COMPATIBILIZER 
This research is a joint project between the University of Twente and Prince of Songkla University, sponsored by the Netherlands Natural Rubber Foundation.

\section{Graduation committee}

Chairman:

Prof. Dr. G.P.M.R. Dewulf

University of Twente, CTW

Secretary:

Prof. Dr. G.P.M.R. Dewulf

University of Twente, CTW

Promotor:

Prof. Dr. Ir. J.W.M. Noordermeer

University of Twente, CTW

Asst. Promotor:

Dr. K. Sahakaro

University of Twente,CTW and

Prince of Songkla University,

Science and Technology

Members:

Prof. Dr. Ir. D.J. Schipper

University of Twente, CTW

Dr. Ir. P.J. Dijkstra

University of Twente, TNW

Prof. Dr. U. Giese

University of Hanover, DIK

Hanover, Germany

Prof. Dr. J. Vourinen

Tempere University of

Technology, Finland

Referees:

Dr. Ir. L.A.E.M. Reuvekamp

Apollo Tyres Global R\&D,

Enschede

Dr. A.V. Chapman

Tun Abdul Razak Research

Centre, UK

Silica-reinforced natural rubber: use of natural rubber grafted with chemical functionalities as compatibilizer

By Karnda Sengloyluan

Ph.D. Thesis, University of Twente, Enschede, the Netherlands, and Prince of Songkla University, Pattani Campus, Thailand, 2015.

Copyright $\odot$ Karnda Sengloyluan, 2015.

All rights reserved.

Cover design by Karnda Sengloyluan

Printed at Wöhrmann Print Service, PO Box 92, 7200 AB Zutphen, the Netherlands.

ISBN: 978-90-365-3898-5

DOI: $10.3990 / 1.9789036538985$

USL: http://dx.doi.org/10.3990/1.9789036538985 


\title{
SILICA-REINFORCED NATURAL RUBBER
}

\author{
USE OF NATURAL RUBBER GRAFTED WITH CHEMICAL \\ FUNCTIONALITIES AS COMPATIBILIZER
}

\section{DISSERTATION}

\author{
to obtain \\ the degree of doctor at the University of Twente, \\ on the authority of the rector magnificus, \\ Prof. Dr. H. Brinksma,
}

on account of the decision of the graduation committee,

to be publicly defended

on Thursday, June $25^{\text {th }}, 2015$ at 14.45

by

Karnda Sengloyluan

born on November $29^{\text {th }}, 1985$

in Satun, Thailand 
This dissertation has been approved by:

Promotor

: Prof. Dr. Ir. J.W.M. Noordermeer

Assistant Promotor

: Dr. K. Sahakaro 
"Defeat is not the worst of failures. Not to have tired is the true failure."

----- "George E. Woodberry" -----

To my beloved family 

Chapter 1 Introduction 1

Chapter 2 Literature overview: reinforcement efficiency of silica-filled 7 rubber with different compatibilizing techniques

Chapter 3 Silica-reinforced tire tread compounds compatibilized by using epoxidized natural rubber

Chapter 4 Silica-reinforced natural rubber compounds

compatibilized by ENR in combination with TESPT and sulfur compensation

Chapter 5 Silica-reinforced natural rubber compounds compatibilized by ENR in combination with different silane coupling agent types

Chapter 6 Influence of types of silane coupling agents on the reinforcement of silica in natural rubber compounds

Chapter 7 Preparation and characterization of silane-grafted natural rubber

Chapter 8 Silane-grafted natural rubber as compatibilizer in silicareinforced natural rubber

Chapter 9 Compatibilization of silica-filled natural rubber by using silane-grafted-natural rubber with compensation of sulfur

Summary

Samenvatting

Symbols and abbreviations

Bibliography

Acknowledgements 



\section{Introduction}

\subsection{Development of silica technology for tires}

A major development of tires was first reported in 1846 when R.W. Thomson invented an elastomeric air tube or "pneumatic tire" to be fixed onto a wheel to reduce the power to haul a carriage and wheeling noise. The pneumatic tire was successfully used for tricycles and bicycles when this concept was reinvented by J.B. Dunlop in 1888. ${ }^{[1,2]}$ The early development of the pneumatic tire was meant only for bicycles, later on it was mainly used for automobiles or motorcars. For decades, the chemicals and processing technology involved in rubber compounds were continuously improved, and both World Wars were important factors to accelerate tire design and development including the introduction of synthetic rubbers and reinforcement materials. ${ }^{[3]}$

The discovery of the vulcanization reaction by Charles Goodyear in $1839^{[2]}$ and the development of rubber tires lead to a continuous consumption increase of rubber and reinforcement materials. The technology of reinforcing fillers was one of the important factors to accelerate the growth of the rubber industry. Fillers such as chalk and china clay, were originally used to reduce production costs, but they also improved the processing behavior of rubber compounds such as reduced die swell and smoothening of the extruded and calendered products. ${ }^{[4]}$ Carbon black was primarily used as a coloring pigment until the reinforcement effect of carbon black was discovered by S.C. Mote in $1904^{[2]}$ in line with the development of rubber tire technology.

Carbon black has a graphite structure with a small amount of functional groups, e.g. phenol, ketone, lactone, carboxyl, etc, on the surface. It can easily be incorporated into rubber and forms filler-rubber interactions. ${ }^{[5]}$ The use of carbon black in rubber compounds leads to an improvement of mechanical properties such as abrasion resistance and tensile and tear strengths. ${ }^{[6]}$ The properties of the filled rubber are mainly affected by filler dispersion and distribution, carbon black characteristics and filler-rubber interactions. ${ }^{[7]}$ Carbon black has a good compatibility with hydrocarbon rubbers, but is limited in use for only black applications. In 1951, the first commercial silica product was marketed under the trade name of Ultrasil VN3 in Europe. Silica was initially used in light- 
colored or transparent articles, e.g., shoe soles. For tire compounds, precipitated silica was first used in small amounts in combination with carbon black to improve adhesion in tire cord-rubber, and to improve cutting and chipping resistances. ${ }^{[8]}$ After a patent of Michelin on the so-called "Green Tire"[?] , silica-silane technology has been increasingly used for passenger car tire treads. Silica is an inorganic filler with hydroxyl, also called silanol groups on its surface that can form hydrogen bonds and thus strong filler-filler interactions to generate silica aggregates and agglomerates. It is therefore difficult to disperse and does not interact with non-polar rubber matrices like natural rubber (NR) and styrene-butadiene rubber (SBR), which are the types of rubbers used in tire compounds. To improve the properties of silica-filled compounds, silane coupling agents are generally added to improve silica-rubber interaction and to decrease the silica-silica interaction.

The use of silane coupling agents leads to reduction of compound viscosity, an improvement in cure characteristics and a significant increase of modulus, tensile strength, and abrasion resistance. The first silane coupling agent introduced by Union Carbide, was mercaptopropyl-trimethoxysilane (MPS) under the trade name of A-189. Afterwards, Bis-(triethoxysilylpropyl)tetrasulfide (TESPT) was introduced under the trade name Si-69 by Degussa. ${ }^{[10]}$ The use of the silica/TESPT combination in tire tread compounds improves wet traction and rolling resistance without negative affects on abrasion resistance. Silica technology has been further developed to improve the properties of silica-filled compounds, either in aspects of the silica characteristics or development of new silane coupling agents or compatibilizer systems.

\subsection{Background of the thesis}

Natural rubber (NR) is an important material to produce rubber tires, especially heavy duty truck tires. NR has superior mechanical properties over other synthetic rubbers, due to its high molecular weight, and regular structure of cis-1,4-polyisoprene. NR can crystallize when it is stretched by external force, so called 'strain-induced crystallization', which leads to high tensile strength and elongation at break. Despite the good strength of NR, reinforcing fillers such as carbon black and silica are commonly used to enhance the properties of NR further for high performance products such as tires. Due to the strong filler-filler interactions between silica aggregates caused by the large number of silanol groups on the silica surface, the use of silica in NR or other hydrocarbon rubbers requires a silane coupling agent or compatibilizer. The most commonly used silane coupling agent is TESPT, but some drawbacks of the use of this silane in silica-filled compounds have been reported. These are an increase of Mooney viscosity upon storage 
because of self-crosslinking between sulfur in the silane structure and rubber molecules, leading to less processability of the compound, ${ }^{[11]}$ and low scorch safety due to breaking of the sulfur-sulfur bonds in the TESPT molecules during mixing at high temperature. Bis(triethoxysilylpropyl)disulfide (TESPD) can be used as alternative to obtain a better scorch safety of silica-filled compounds while providing similar final properties when compared to those of TESPT treated compounds. ${ }^{[12]}$

Silica contains silanol groups on its surface which can interact or react with polar functional groups of some polar rubbers and enhance compatibilization. Acrylonitrile butadiene rubber $(\mathrm{NBR})^{[13]}$ and polychloroprene rubber ${ }^{[14]}$ have been used as compatibilizers in silica-filled compounds to improve silica-rubber interaction, silica dispersion and hence the vulcanizate properties. Chemically modified NRs with polar functional groups such as epoxidized natural rubber $(E N R)^{[15]}$, maleated natural rubber $(\mathrm{MNR})^{[16]}$ have also been used as compatibilizers in silica-filled compounds. The epoxide groups of ENR can interact with silanol groups on the silica surface, leading to a decrease of filler-filler interaction and an increase of the properties of the filled compounds.

\subsection{Aim of the project}

The research project covered in this thesis aimed to increase the compatibility between silica and natural rubber by using chemically functionalized rubber as a compatibilizer, so as to replace or minimize the use of silane coupling agent. The objectives for the research are listed below;

1. To prepare modified natural rubbers by using different types and levels of functional groups which can potentially interact or react with silanol groups on the silica surface.

2. To investigate the effect of modified natural rubber when added as compatibilizer in silica-filled natural rubber on cure characteristics, Mooney viscosity, bound rubber content, mechanical and dynamic properties.

3. To select a suitable type and level of functionality of the modified natural rubber to be used as compatibilizer in silica-filled natural rubber. The mixing parameters are to be optimized to obtain optimum properties of silica-filled natural rubber.

4. To characterize the interaction, level of compatibility and reinforcement of silica-filled natural rubber when the modified rubber is used as compatibilizer, compared to use of a conventional silane coupling agent. 


\subsection{Concept of the thesis}

This project investigated the use of chemically functionalized natural rubbers as compatibilizers in silica-reinforced natural rubber with emphasis on the improvement of silica-rubber interaction, mechanical properties and tire performance, i.e. wet skid and rolling resistance, in comparison with the use of a conventional silane coupling agent. The thesis is composed of the following chapters;

Chapter 1 Introduction: This first chapter shortly reveals the development of tires and filler technology with emphasis on tire compounds. It is then followed by a description of the background and aims of this research.

Chapter 2 Literature overview: This part focuses on the use of reinforcing fillers mainly carbon black and silica in rubber compounds. The reinforcing efficiency of silica, factors influencing silica reinforcement and development of silica technology for tire compounds are reviewed. The use of silane coupling agents, some polar materials and chemically modified rubbers to improve silica-rubber interactions and the properties of silica-filled compounds are discussed. This chapter ends by providing motivation and scope of the project.

Chapter 3 Silica-reinforced tire tread compounds compatibilized by using epoxidized natural rubber: This chapter first describes the preparation and characterization of epoxidized natural rubber (ENR) with various mole\% of epoxide. ENRs with different mole\% of epoxide groups are used as compatibilizers to optimize the properties of silica-filled natural rubber compounds compared to compounds with Bis(triethoxysilylpropyl)tetrasulfide (TESPT) and without compatibilizer.

Chapter 4 Silica-reinforced natural rubber compounds compatibilized by ENR in combination with TESPT and sulfur compensation: While a silica-filled natural rubber compound with only ENR as compatibilizer shows overall lower properties than with TESPT silane coupling agent, this section studies the use of an optimized ENR type and content (i.e. 7.5 phr of ENR-51) in combination with TESPT to further enhance the properties of the compounds. The TESPT contents are varied and the effect of extra sulfur to compensate for the sulfur contents in TESPT molecules on the properties of silicareinforced natural rubber is investigated.

Chapter 5 Silica-reinforced natural rubber compounds compatibilized by ENR in combination with different silane coupling agent types: The reinforcing 
efficiency of silica-filled NR compounds compatibilized with three different silane types, i.e. TESPT, 3-mercaptopropyl-di(tridecan-1-oxy-13-penta(ethyleneoxide))ethoxysilane (VP Si363), and 3-octanoyl-thio-propyltriethoxysilane (NXT), is discussed. Then, these different silane types together with ENR-51 at $7.5 \mathrm{phr}$ are used as compatibilizers in silica-filled NR compounds. The properties of such compounds are studied in comparison with the reference compound containing the optimized conventional TESPT silane coupling agent.

Chapter 6 Influence of types of silane coupling agents on the reinforcement of silica in natural rubber compounds: The effect of different types of silane coupling agents on the properties of silica-filled NR compounds is studied by using four types of silane; TESPT, NXT, VP Si-363 and vinyltriethoxysilane (VTES) that are used based on molar and ethoxy functional group equivalents. The properties of the silica-filled NR compounds and vulcanizates are comparatively investigated in order to find a grafting silane candidate to be used later for preparation of silane-grafted NR.

Chapter 7 Preparation and characterization of silane-grafted-natural rubber:

This section focuses on the preparation and characterization of silane-grafted-natural rubber. Silanes with mercapto function: VP Si-363, and with blocked mercapto function: NXT are used to graft onto the natural rubber molecules in the melt mixing state in an internal mixer. The preparation conditions are optimized and the products are characterized by Fourier-transform infrared spectroscopy (FT-IR), proton nuclear magnetic resonance spectroscopy $\left({ }^{1} \mathrm{H}-\mathrm{NMR}\right)$ and elemental analysis.

Chapter 8 Silane-grafted-natural rubber as compatibilizer in silicareinforced natural rubber: The use of NXT- and VP Si-363-grafted natural rubbers as compatibilizers in the silica-reinforced natural rubber compounds are studied in comparison with the straight use of silane. The properties of the silica-reinforced natural rubber are studied in terms of filler-filler and filler-rubber interactions, mechanical and dynamic mechanical properties by taking the compound with optimum amount of TESPT as a reference.

Chapter 9 Compatibilization of silica-filled natural rubber by using silanegrafted-rubber with compensation of sulfur: Due to the difference in total sulfur content in the compounds, this chapter focuses on the further improvement of the use of silanegrafted-natural rubber as compatibilizer by using extra sulfur to compensate for sulfur atoms released from the TESPT molecules. Overall properties are assessed and discussed in comparison with the use of the conventional TESPT silane coupling agent. 
Summary: This chapter summarizes all the findings and knowledge derived from the experimental studies.

\subsection{References}

1. C.M. Blow, C. Hepburn, "Rubber Technology and Manufacture", Butterworths, London, second edition, 1982.

2. J.E. Mark, B. Erman, F.R. Eirich, "Science and Technology of Rubber", Academic Press, San Diego, second edition, 1994.

3. T. French, "Tire Technology", IOP Publishing, 1988.

4. J.A. Brydson, "Rubber Materials and Their Compounds", Elsevier Applied Science Publishers, England, 1988.

5. S. Woff, Rubber Chem. Technol., 69, 325 (1996).

6. Z. Rigbi, Adv. Polym. Sci., 36, 21 (1980).

7. L. Kasasek, M. Sumita, J. Mater. Sci., 31, 281 (1996).

8. B. Schwaiger, A. Blume, Rubber World, 222, 32 (2000).

9. R. Rauline, EP Patent 0501227A1, to Michelin \& Cie, February 9, 1992.

10. F.W. Barlow, "Rubber Compounding: Principles, Materials, and Techniques", Marcel Dekker, New York, 1988.

11. C.J. Lin, W.L. Hergenrother, A.S. Hilson, Rubber Chem. Technol., 75, 215 (2002).

12. J.W. ten Brinke, P.J. van Swaaij, L.A.E.M. Reuvekamp, J.W.M. Noordermeer, Rubber Chem. Technol., 75, 12 (2003).

13. S.-S. Choi, J. Appl. Polym. Sci., 79, 1127 (2001).

14. A. Das, S.C. Debnath, D. De, D.K. Basu, J. Appl. Polym. Sci., 93, 196 (2004).

15. K.M. George, J.K. Varkey, K.T. Thomas, N.M. Mathew, J. Appl. Polym. Sci., 85, 292 (2002).

16. K. Sahakaro, S. Beraheng, J. Appl. Polym. Sci., 109, 3839 (2008). 


\section{Literature Overview: Reinforcement Efficiency of Silica-Filled Rubber with Different Compatibilizing Techniques}

This Chapter gives an overview of reinforcing fillers which have been used in the rubber industry with particular attention to fillers used in tire compounds, e.g. carbon black and silica. Filler characteristics, such as specific surface area, filler structure and surface activity, that influence the properties of filled rubber are discussed. Filler reinforcement of rubbers in general, and dynamic properties of filled rubber in relation to tire performance are reviewed. Silica-reinforced rubber compounding and its associated difficulties coming from silica-rubber incompatibility are addressed. This leads to the development of silicasilane technology which involves a silanization reaction between alkoxy groups of a silane and silanol groups on the silica surface. Due to the greatly increased interest in silica usage especially for low rolling resistance tire treads, several alternative approaches have been adopted in order to improve silica dispersion and filler-rubber interaction. Some of those approaches are reviewed in this section. Among them the use of polar polymers, such as polychloroprene rubber, acrylonitrile-butadiene rubber, epoxidized rubbers, as compatibilizers for silica-reinforced rubber compounds. Particular attention is given to chemically modified natural rubber which can potentially be used for silica-reinforced natural rubber compounds, as investigated in this thesis. The chapter ends by providing the motivation for this thesis. 


\subsection{Introduction}

During the past decades the rubber industry has increasing concerns about its dependence on raw materials which are derived from petroleum. Synthetic rubbers, carbon black, processing and extender oils, and most of the additives for rubber are derived from petrochemical products which are affected by the increasing costs and decreasing supply of crude oil. This situation has stimulated interests in products which are less dependent on petroleum and require less energy to produce. Fillers for rubber compounds that are made from virtually inexhaustible natural sources such as clay, limestone, and talc require less energy to produce than the synthetic fillers like carbon black, silicates and precipitated silica, but these are classified as non- to low-reinforcing and have high specific gravities compared to carbon black and silica. Commercial applications of elastomers often require the use of active particulate fillers to obtain a certain level of reinforcement and product performance. In the rubber industry, beside carbon black, silica is an important white reinforcing filler often used to impart specific properties to the rubber compounds. It is well known that the filled-rubber compounds are multiphase composites, in which several factors play a role on the reinforcement efficiency.

In 1951, the first commercial silica product was marketed under the trade-name of Ultrasil VN3 in Europe. Silica was initially used in light-colored or transparent articles, e.g., shoe soles. Precipitated silica was also used in small amounts in tire treads for commercial vehicles to improve tear propagation resistance. ${ }^{[1]}$ In 1990 s, the European tire manufacturer Michelin introduced passenger car tires with treads formulated by incorporating silica as reinforcing filler instead of conventional carbon black. The tires with silica-filled tire tread compounds were claimed as "Green Tires" due to their lower rolling resistance and heat build-up, when compared with conventional tires with treads filled with carbon black. ${ }^{[2]}$ However, the surface functional environment of silica particles is different from that of carbon black due to the existence of the hydrophilic silanol groups on the surface. Thus, hydrocarbon rubbers like natural rubber (NR) and styrene butadiene rubber (SBR) are not compatible with silica, and their compounds without compatibilizer or coupling agent usually have inferior mechanical and physical properties as a result of poor interfacial adhesion.

\subsection{Reinforcing fillers}

Several types of fillers are in use in rubber compounds for different purposes, such as for reduction of production costs or to improve some properties like processing or 
vulcanizate properties, or both. The fillers can be classified by their particle sizes as shown in Figure 2.1. ${ }^{[3]}$

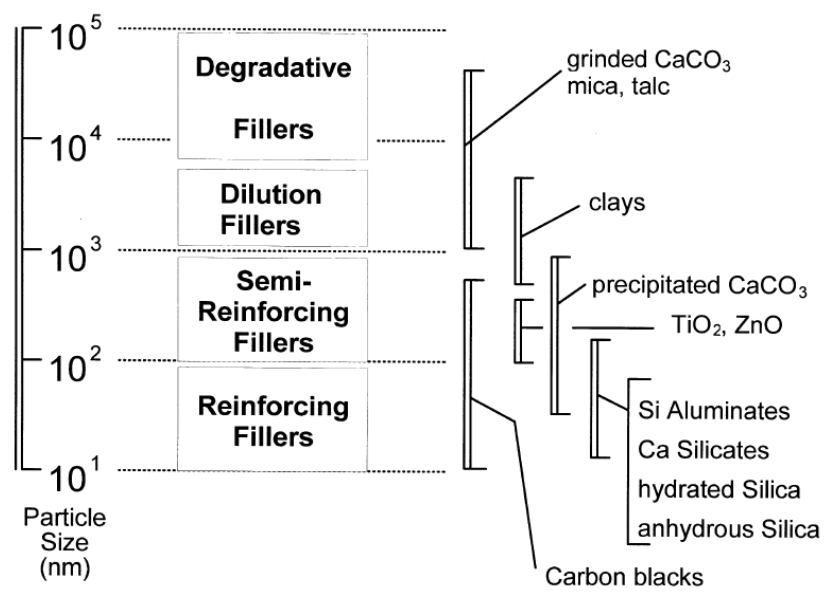

Figure 2.1 Classification of fillers on basis of particle sizes. ${ }^{[3]}$

\subsubsection{Filler characteristics}

Reinforcement basically relates to composites built from two or more components which have different mechanical characteristics. The strength of one of these components is imparted to the composite combined with the set of favorable properties of another component. Reinforcement of rubber by fillers relates to the improvement of modulus and failure properties, such as tensile strength, tear and abrasion resistances of the vulcanizates. The energy at rupture which can be obtained from a stress-strain curve may be regarded as the best single criterion for reinforcement. ${ }^{[4,5]}$

The reinforcement of rubber with fillers is dependent on their specific characters. The important factors that affect the reinforcing efficiency are ${ }^{[5,6]}$

1. Specific surface area and particle size - The primary particle size of a filler is related to its specific surface area and can be determined directly by using electron microscopy. Alternatively, it can be characterized by different adsorption methods. The nitrogen adsorption method according to ASTM D6556, the so-called BET (BrunauerEmmett-Teller) method measures the total surface area including micro-porosity of the particles. In this case, the nitrogen surface area (NSA) and the statistical thickness surface area (STSA) can be determined. Another method is based on the cetyltrimethyl ammonium bromide (CTAB) adsorption which analyzes only the external surface area of 
filler particles or aggregates which can be related to the contact area between filler and rubber molecules, excluding the micro-pores in which they cannot penetrate. The CTABadsorption on silica surface is measured according to ASTM D6845. It should be noted that the CTAB-method for the measurement of carbon black surface area according to ASTM D3765 has been withdrawn due to its poor testing precision and labor intensiveness. ${ }^{[7]}$ For the specific surface area of carbon black which is accessible for rubber chains, the STSA technique is applied. ${ }^{[7]}$ Decrease of filler particle size leads to an increase of surface area which normally has a positive effect on rubber reinforcement.

2. Filler structure - Generally, fillers do not appear in individual primary particles form, but preferably in cluster forms of aggregates or agglomerates, depending on how strong the filler-filler interactions are. Carbon black can form filler structure through Van der Waals forces which are a weaker interaction than hydrogen bonding between silica particles. The empty space between aggregates (or agglomerates) of a filler can be determined by the volume of dibutylphthalate (DBP) absorption according to ASTM D6845-03, which number is used to indicate filler structure. The "structure" of a filler relates to the aggregate structure size and density. Due to toxicity of DBP, the oil adsorption number of carbon black was implemented according to ASTM D2414-02a. Paraffinic oil is preferentially used offering the advantage of being non-hazardous. Filler structure has an influence on reinforcing efficiency and the addition of high-structure carbon black into rubber leads also to an increase of rubber vulcanizate properties.

3. Surface activity - The carbon black surface consists of a small amount of different chemical functional groups such as carboxyl, quinone, lactone, phenol and hydroxyl groups, causing a difference in capacity and absorption energy. The limited number of polar functional groups does not essentially contribute to the reinforcement of carbon black in non-polar rubbers. The surface activity of carbon black mainly refers to the strength of interactions between the carbon black surface and rubber via either physical or chemical adsorptions or mechanical interlocking which have an important effect on modulus, hysteresis, abrasion resistance and other mechanical/physical properties. ${ }^{[8]}$ The surface activity of carbon black can be influenced by heat treatment at a high temperature of 1600 to $3000^{\circ} \mathrm{C}$, causing a rearrangement of nano-crystallites to be more ordered in building primary particles of carbon black. ${ }^{[3]}$ In case of inorganic silica with a large number of hydroxyl groups on its surface, causing strong filler-filler interactions, it is essential to use a silane coupling agent to create a bridge between silica and rubber. 


\subsubsection{Carbon black}

Carbon black is composed of aggregated particles of elemental carbon which are partly graphitic in structure. The carbon atoms in the particles are oriented in layered planes which, by parallel alignment and overlapping, give the particles their semi-graphitic nature. The outer layers are more graphitic than those in the center. The particle size ranges from 10 to $400 \mathrm{~nm}$ in diameter, wherein the smaller ones are less graphitic. Carbon blacks are produced by converting either liquid or gaseous hydrocarbon to carbon and hydrogen by combustion or thermal decomposition. ${ }^{[9]}$ The most important characteristic of carbon black is its external specific surface area. High area is associated with a high level of reinforcement, but at the expense of high cost, high hysteresis and more difficult processing. The second most important property is its "structure", which refers to the bulkiness of the carbon black aggregates. In general, high structure or high bulkiness carbon black is associated with a large number of carbon black primary particles per aggregate. ${ }^{[10]}$ It is well-known that filler-rubber interactions depend upon the compatibility between filler and rubber matrix. Carbon black is a filler that can easily be incorporated into rubber, generates bound rubber and is compatible with hydrocarbon rubbers including styrene butadiene rubber (SBR), natural rubber (NR), polybutadiene rubber (BR) and isobutylene isoprene rubber (IIR). The carbon black surface contains only a small number of functional groups as shown in Figure 2.2, making it relatively non-polar and easy to bound to rubber. The adsorption of rubber on carbon black forming bound rubber readily occurs during mixing and also after mixing. ${ }^{[8]}$

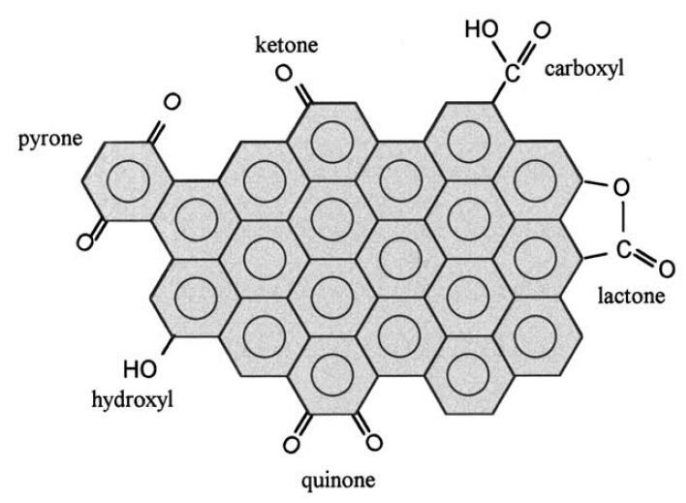

Figure 2.2 Functional groups on carbon black surfaces. ${ }^{[3]}$ 
The functional groups on carbon black surfaces can react with ethoxy groups of Bis-(triethoxysilylpropyl)tetrasulfide (TESPT) and become an activated carbon black surface. Those activated carbon black surface can then form covalent bonds with rubber and results in a reduction of compound hysteresis. ${ }^{[11]}$

\subsubsection{Silica}

In 1939, the first reinforcing siliceous filler was introduced. ${ }^{[9]}$ A calcium silicate was prepared by wet precipitation from a sodium silicate solution with calcium chloride. In further development of the process, the calcium was leached out by hydrochloric acid to yield a reinforcing silica pigment. About ten years later, a direct precipitated silica from sodium silicate solution was developed to a commercial process and this became the major process of today. In 1950, different types of anhydrous silica which were made by reacting silicium-tetrachloride or "silicon chloroform" with water vapor in a hydrogenoxygen flame were produced. These pyrogenic silicas formed at high temperature (about $1400^{\circ} \mathrm{C}$ ) have a lower concentration of hydroxyl groups on the surface than the precipitated silica. The precipitated silica contains about $85-90 \%$ silica and has ignition losses of $10-14 \%$, whereas the pyrogenic silica contains $99.8 \%$ silica. $^{[4]}$

Amorphous silica consists of silicium and oxygen tetrahedrally bonded into an imperfect three dimensional structure. Silica contains a large number of silanol groups on its surface, and these polar silanol groups lead to a hydrophilic surface. Thus, silica is not compatible with hydrophobic rubbers. Silica particles form strong filler-filler interactions with other particles to generate aggregates and agglomerates. The silanol concentration on a silica surface depends on the number of silicium atoms per area the surface and the number of hydroxyl groups present on each silicium atom. The surface silanol-group content of Zeosil 1165MP, a typical easy-dispersion silica type used for silica-filled passenger tires, was reported to be $4.90 \mathrm{OH} \cdot \mathrm{nm}^{-2[12]}$ Three types of surface hydroxyl groups; isolated, vicinal (on adjacent silicium atoms) and geminal (two hydroxyl groups on the same silicium atom) ${ }^{[11,13]}$ have been identified, as shown in Figure 2.3.

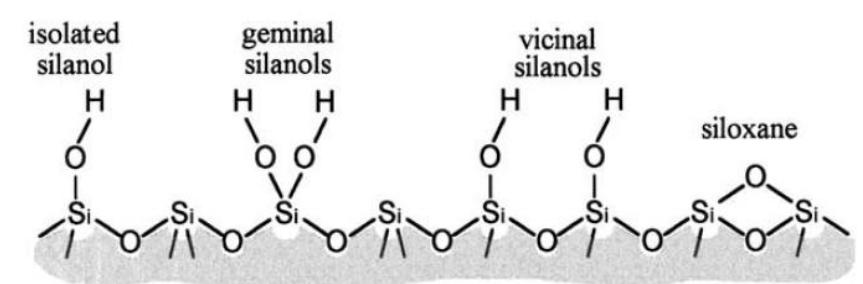

Figure 2.3 Typical silanol groups on a silica surface. ${ }^{[8]}$ 
The surface free energy of fillers, $\gamma_{s}$, can be split in two components which are the dispersive component, $\gamma_{s}^{d}$, and the specific component, $\gamma_{s}^{s p}$, as follows; $;^{[14,15]}$

$$
\gamma=\gamma_{s}^{d}+\gamma_{s}^{s p}
$$

The dispersive component indicates the tendency of adhesion to organic molecules such as polymers, and the specific component indicates the tendency of interaction with itself and polar components. When compared to carbon black, silica has a low dispersive component but a high specific component of free energy. This results in a poor interaction between silica and hydrocarbon rubbers but a high degree of silica agglomeration.

The surface energy of silica has been characterized by techniques, such as the inverse gas chromatography (IGC) technique. ${ }^{[14,15]}$ In the IGC-experiment the filler is used as the stationary phase, and the solute probe is injected. Based on the adsorption of several solutes on the silica surface and filler-probe interactions, the surface energy of silica and its estimated interactions with rubbers are reported. The specific interactions of the probes, analogs of elastomers, with silica decrease in the order: nitriles $>$ aromatic hydrocarbon $>$ olefins $>n$-alkanes $>$ isoalkanes. Thus, the interactions between elastomers with silicas, which indicates their compatibility, can be ranked in order as; ${ }^{[14]}$

$$
\mathrm{NBR}>\mathrm{SBR}>\mathrm{NR} \geq \mathrm{BR}>\mathrm{HV}-\mathrm{BR}>\mathrm{EPM}>\mathrm{IIR}
$$

where NBR is acrylonitrile butadiene rubber, SBR is styrene butadiene rubber, NR is natural rubber, BR is polybutadiene rubber, HV-BR is high-vinyl polybutadiene rubber, EPM is ethylene propylene rubber and IIR is isobutylene isoprene rubber or commonly known as butyl-rubber.

\subsubsection{Carbon-silica dual phase fillers}

Lately, filler technology for tire tread compounds has been focused on new filler systems and silane coupling agents or functionalized fillers to improve tire rolling resistance, abrasion resistance and wet traction. A new filler system, so called carbonsilica dual phase filler (CSDPF) has been presented in the market under the trade name of $\mathrm{ECOBLACK}^{\mathrm{TM}}$ by Cabot Corporation. From the results of electron spectroscopy for chemical analysis (ESCA) and infrared spectroscopy (IR), CSDPF is in the form of individual composite aggregates of carbon and silica phases. The silica and carbon phases which differ from simple mixes of carbon black and silica aggregates, are finely dispersed within primary particles to make up the dual phase aggregates. Silica particle 
sizes in the range of 0.4 to $2 \mathrm{~nm}$ were reported after treating the dual phase filler with hydrofluoric acid. ${ }^{[16,17]}$ It has been reported that CSDPF-filled rubber exhibits a lower Payne effect when compared to carbon black and silica/silane, which is related to filler networking in the matrix ${ }^{[18]}$ and a higher dispersive component of the surface energies of the CSDPF leading to a higher polymer-filler interaction in low polar or non-polar rubbers. ${ }^{[19]}$ The decrease of elastic modulus $\left(G^{\prime}\right)$ with strain amplitude, or Payne effect ${ }^{[20]}$ of NR compounds filled with CSDPF in comparison with other fillers is shown in Figure 2.4. This filler type has higher active carbon and hydrogen groups, and is more acidic than pure carbon black leading to significantly more reactive functional groups available to react with a silane coupling agent. ${ }^{[21]}$ CSDPF-filled rubber tire tread compounds with TESPT silane coupling agent are claimed to show superior performance over conventional fillers, i.e. carbon black and silica, due to higher rubber-filler interaction and less filler-filler interaction. Wang et al. ${ }^{[22]}$ demonstrated that the use of CSDPF with TESPT shows better abrasion resistance and more than $40 \%$ reduction in tan $\delta$ at $70^{\circ} \mathrm{C}$ which correlates with rolling resistance of tire treads made thereof when compared to carbon black-filled compounds. In addition, the CSDPF-filled compounds show superior abrasion resistance and tear strength over silica-filled compounds, and better wet skid resistance of tire treads than conventional fillers.

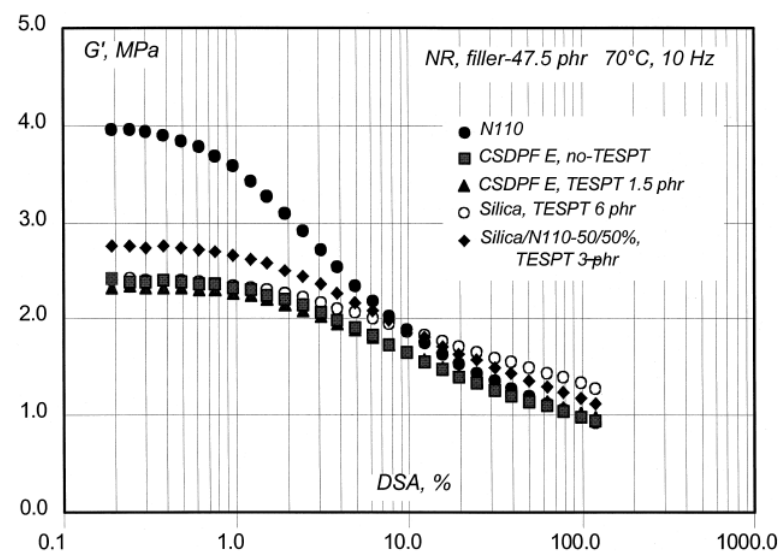

Figure 2.4 Elastic modulus as a function of strain amplitude of truck tire tread compounds with various fillers. ${ }^{[22]}$ 


\subsection{Filler reinforcement of rubbers}

\subsubsection{Hydrodynamic effect}

The viscosity of liquids or modulus of elastomeric matrices is increased when rigid particles are added. At low filler loadings, the modulus of filled rubber increases linearly with the volume fraction of filler $(\phi)$. This can be explained by the first equation proposed by Einstein. ${ }^{[23]}$

$$
E=E_{0}(1+2.5 \phi)
$$

Where $E_{0}$ is the Young's modulus of unfilled rubber, and $E$ is the Young's modulus of filled rubber.

At high filler loading, the linear relation with $\phi$ does no longer hold. The GuthGold equation is then used to explain the modulus of filled rubber by adding a second order term $\phi^{2}$ which accounts for the interactions between particles in a denser state. The equation reads as follows;

$$
E=E_{0}\left(1+2.5 \phi+14.1 \phi^{2}\right)
$$

Smallwood demonstrated the equivalence of the concentration dependence of viscosity and modulus for filled rubber. Therefore, this equation is also often called the Einstein-Guth-Smallwood model. ${ }^{[24]}$

\subsubsection{Payne effect}

The addition of filler to rubber compounds has a strong impact on the static and dynamic behavior of such rubbers. Figure 2.6 shows the typical elastic modulus of filled rubber-samples versus dynamic strain. Besides the strain-independent contributions of the hydrodynamic effect, filler-to-rubber interaction and crosslinks or the network in the rubber matrix, the elastic modulus shows a strong strain-dependence at low strains. This stress softening at low deformation is known as the Payne effect. ${ }^{\text {[20] }}$

Therefore, the following effects contribute to the storage modulus; ${ }^{[6]}$

a) Rubber network: the network depends on the nature of the rubber and crosslink density in the rubber matrix, involving either physical or chemical linkages or both. 
b) Hydrodynamic effect: this effect results from the fact that filler is a rigid phase, which cannot be deformed. It can be described by the Guth, Gold and Smallwood equation. ${ }^{[24]}$

c) Filler-rubber interaction: or 'in-rubber structure' depends on a combination of the structure of the filler in the in-rubber state and the extent of filler-rubber interactions which can be attributed to physical forces as well as to chemical linkages or a combination of both. In the case of a silica-silane system, this effect arises from chemical linkages between rubber and silica by silane bridging.

d) Filler-filler interaction: the stress softening at low strain is attributed to a breakdown of filler-filler bonds. ${ }^{[25]}$ This behavior is called the Payne effect, which plays an important role for understanding the reinforcement mechanism in filled rubber. The strong filler-filler interaction in silica-filled rubber is mainly caused by hydrogen bonds between silica particles. The filler network can be easily destroyed at low strain or low deformation, leading to a decrease of elastic modulus of the filled rubber.

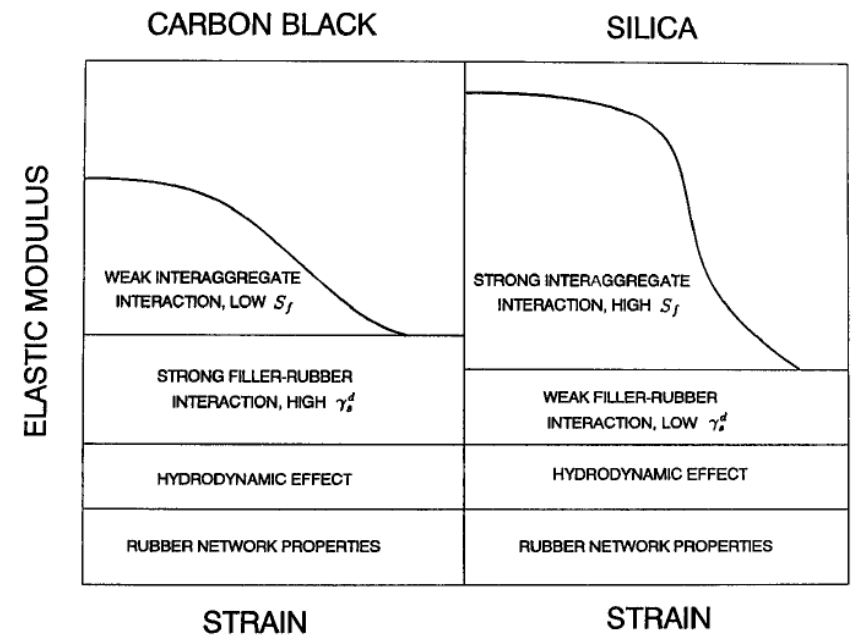

Figure 2.5 Payne effect of carbon black and silica-reinforced rubber. ${ }^{[24]}$

It is clearly seen in Figure 2.5 that silica-filled rubber has a weaker filler-rubber interaction but much stronger filler-filler interaction when compared with carbon black-filled compounds for reason of their different surface free energies. 


\subsubsection{Dynamic properties in relation to tire performance}

The viscoelastic behavior of elastomers can be monitored by applying a sinusoidal stress of frequency $\omega$, as shown in Figure 2.6. When such sinusoidal shear stress is applied to the viscoelastic material, the strain will show a sinusoidal response but out of phase.

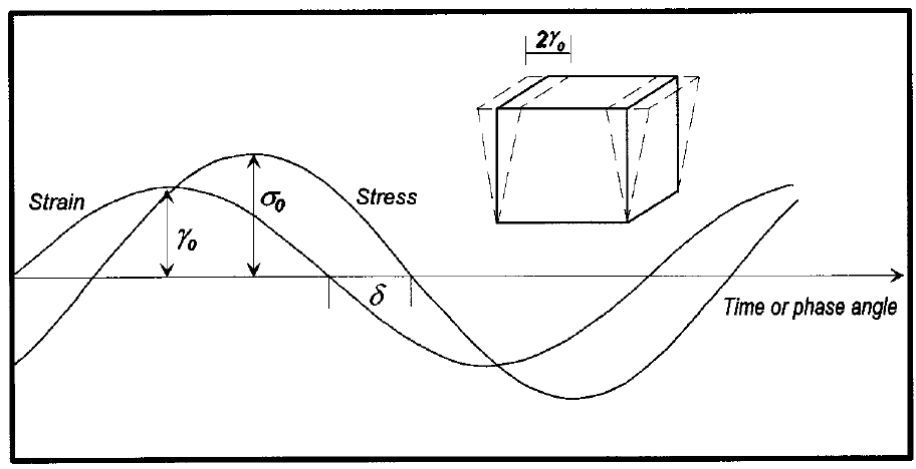

Figure 2.6 Sinusoidal response of viscoelastic materials. ${ }^{[26]}$

The correlation between stress, $\sigma$, and strain, $\gamma$, can be written as;

$$
\gamma=\gamma_{0} \sin \omega t
$$

and

$$
\sigma=\sigma_{0} \sin (\omega t+\delta)
$$

where $t$ is time, $\delta$ is the phase angle between stress and strain, $\gamma_{0}$ and $\sigma_{0}$ are the maximum amplitudes of strain and stress, respectively. Then, the stress can be decomposed into two components, i.e. one in-phase and one out-of-phase with strain;

$$
\sigma=\sigma_{0} \sin \omega t \cos \delta+\sigma_{0} \cos \omega t \sin \delta
$$

The dynamic stress-strain behavior of the elastic material is then expressed in the storage modulus, $G^{\prime}$, in phase with strain, and the loss modulus, $G^{\prime \prime}$, out of phase;

$$
\sigma=\gamma_{0} G^{\prime} \sin \omega t+\gamma_{0} G^{\prime \prime} \cos \omega t
$$

with

$$
G^{\prime}=\left(\sigma_{0} / \gamma_{0}\right) \cos \delta
$$


and

$$
G^{\prime \prime}=\left(\sigma_{0} / \gamma_{0}\right) \sin \delta
$$

Thus

$$
\tan \delta=G^{\prime \prime} / G^{\prime}
$$

Alternatively, in a cyclic strain test the shear modulus can be expressed in the complex modulus $G *$ as;

$$
G^{*}=\left(\sigma_{0} / \gamma_{0}\right)=G^{\prime}+i G^{\prime \prime}
$$

The addition of fillers into polymers causes a change of the dynamic mechanical properties of the materials. The energy loss of rubbery materials during dynamic strain correlates with heat generation and fatigue life, which have an influence on tire rolling resistance, wet traction and abrasion resistance. The rolling resistance is related to the response of rubber at a frequency of around $10 \mathrm{~Hz}$ and a temperature range of 50 to $80^{\circ} \mathrm{C}$. In case of wet grip or wet traction, it is the stress which is generated by the skidresistance between the surface of the tire tread and the road surface. The frequency of this movement depends on the roughness of the road surface. For good wet grip, the energy loss should be high around $10^{4}$ to $10^{7} \mathrm{~Hz}$ in the same temperature range of 50 to $80^{\circ} \mathrm{C}$. By virtue of the time-temperature superposition principle the viscoelastic behavior at $10^{4}-10^{7} \mathrm{~Hz}$ and $50-80^{\circ} \mathrm{C}$, is equivalent to approximately $10 \mathrm{~Hz}$ at $-20-+20^{\circ} \mathrm{C}$. The loss tangent as a function of temperature at a frequency of $1-10 \mathrm{~Hz}$ can therefore be used to imply the viscoelastic properties of tire compounds as shown in Figure 2.7. ${ }^{[18,26]}$

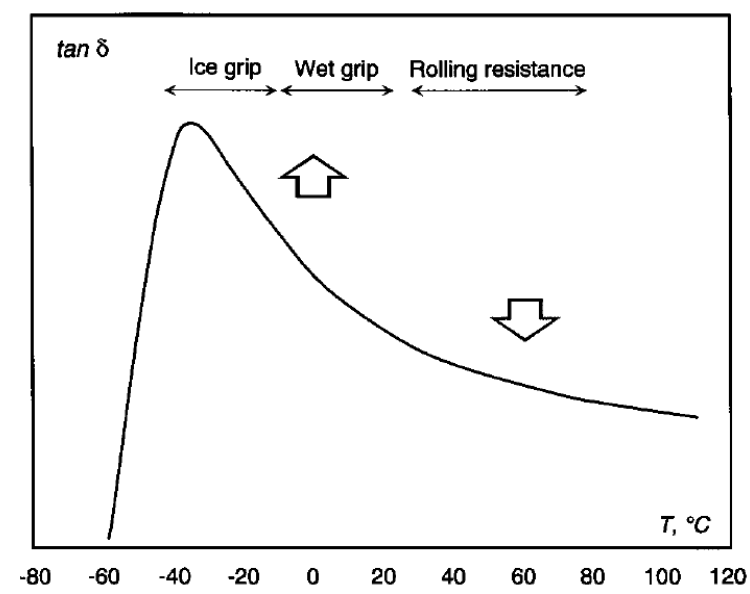

Figure 2.7 Tan $\delta$ versus temperature at $1-10 \mathrm{~Hz}$ related to different dynamic properties of tire performances. ${ }^{[26]}$ 
From the viscoelastic properties, the ideal tire material should have a low tan $\delta$ at $50-80^{\circ} \mathrm{C}$ in order to reduce rolling resistance and save driving energy, while the tan $\delta$ value should be high at low temperature to provide good wet skid resistance, wet grip or wet traction and ice grip.

The addition of fillers into rubber compounds has a strong effect on the static and dynamic properties of the rubbers. The combined effects of the different polymer network, filler-filler interactions, the hydrodynamic effect and in-rubber structure or filler-polymer interaction show a unique influence on the dynamic properties. The complex modulus of filled rubber at low and high strain deformation can be related to two different types of interactions; ${ }^{[6]}$

\section{a) Low strain deformation ( $<<5 \%)$}

The complex modulus at low strain indicates the filler-filler network. High filler loading and increasing specific surface area, meaning decreasing primary particle size of filler, lead to a significant increase of filler-filler interaction. This effect can be explained by the decrease of the inter-aggregates distance of the filler while its surface area is increased, leading to filler-filler network formation. The filler network has a major effect on tire rolling resistance, and an increase of filler surface activity or affinity to the rubber polymer leads to a decrease of filler networking because of better filler-rubber interaction.

\section{b) High strain deformation (> 30\%)}

The complex modulus at high strain indicates the in-rubber structure or fillerpolymer interaction. In case of carbon black-filled rubber, the increase of filler structure and surface activity enhances the in-rubber structure. The incorporation of high structure filler increases occluded rubber in the void spaces within the filler aggregates that leads to a high effective filler volume in the matrix. Meanwhile, the surface activity contributes to the physical and chemical interactions between filler and rubber.

\subsection{Silica-reinforced rubber}

\subsubsection{Silane compatibilized silica-filled compounds}

The addition of silica into rubber compounds offers at least two advantages that are reduction of heat build-up and improvement in tear strength, cut, chip and chunking resistance, when compared to the use of carbon black. Silica itself gives a lower degree of reinforcement when compared to carbon black of the same primary particle size due to the different nature of the surface chemistry of the fillers. In general, silica can reinforce better 
in more polar rubbers when compared to non-polar rubbers due to the higher silica-torubber interactions. The poor reinforcement efficiency of silica-filled non-polar rubbers can be improved by using silane coupling agents. The silica-silane coupling agent reinforcement mechanism involves two key reactions: (1) the silanization reaction in which the silane coupling agent reacts with the silica; and (2) the formation of crosslinks between the silane modified silica and rubber. ${ }^{[27,28]}$

\subsubsection{1) Silanization reaction}

The hydrophilic surface of silica is incompatible with a hydrophobic rubber. Silane coupling agents are most widely used to be added into silica-filled compounds to improve compatibility and increase the interactions between silica and rubber. The process involves adsorption of the silane coupling agent onto the silica surface and subsequently a reaction between hydroxyl groups on the silica surface with methoxy- or ethoxy-groups of the silane coupling agent. The chemical reaction between the silica and alkoxy-silyl groups of the silane coupling agent is the so-called "silanization".

The silanization reaction takes place in two steps. The primary step is the reaction of silanol-groups on the silica surface with alkoxy-groups of a silane molecule. There are two possible reaction mechanism involved in this primary step: 1) a direct reaction of silanol groups with the alkoxy group, and 2) hydrolysis of the alkoxy groups followed by a condensation reaction with the silanol groups. The secondary step is a condensation reaction between adjacent molecules of silane coupling agent on the filler surface. Both steps of the silanization reaction are shown in Figure 2.9. ${ }^{\text {[27] }}$

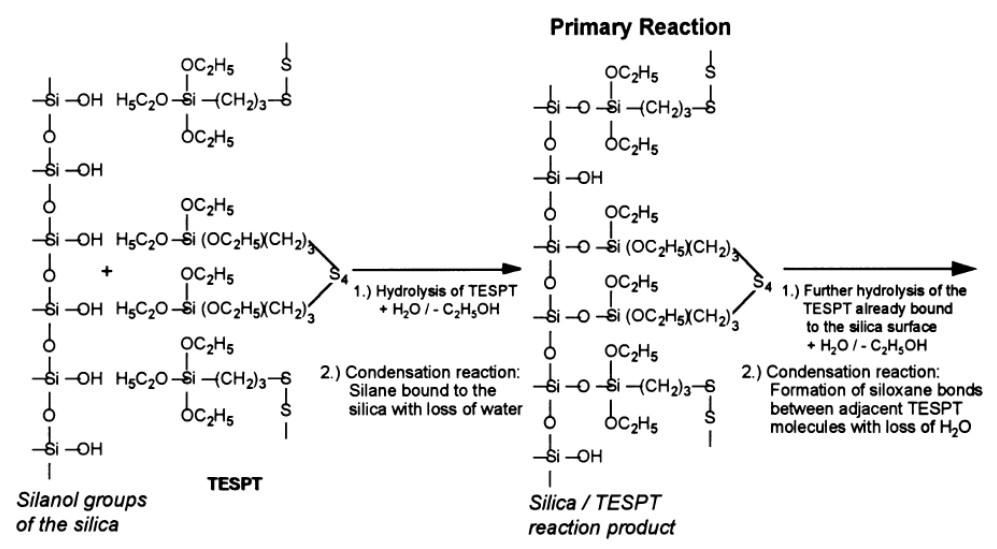

Secondary Reaction

Figure 2.9 Proposed reaction mechanism of the silanization reaction. ${ }^{[27]}$ 
A silane coupling agent that contains sulfur atoms in its structure such as TESPT, can act as a sulfur donor to cause premature crosslinking in the rubber compound during mixing and later-on during the vulcanization reaction. ${ }^{[28]}$ The proposed reaction of TESPT and the silanol groups on the silica surface is shown in Figure 2.9. The silanization reaction of a silica-filled natural rubber compound is optimal at a mixing discharge temperature between $135-150^{\circ} \mathrm{C}{ }^{[29]}$ This reaction can be accelerated by increasing the mixing temperature ${ }^{[28,29]}$, presence of moisture on the silica surface ${ }^{[27]}$ and use of a basic secondary accelerator like diphenylguanidine (DPG). ${ }^{[30]}$

\subsubsection{2) Kinetics of the silanization reaction}

The silanization reaction comprises a primary and a secondary reaction, as shown in Figure 2.9. The reaction between ethoxy-groups on TESPT molecules and silanol groups on the silica surface leads to the release of ethanol molecules. ${ }^{[27]}$

Primary reaction - The evolution of ethanol at 30 to $60^{\circ} \mathrm{C}$ is considered to correspond to the primary reaction by assuming that 1 mole of TESPT can react with 2 moles of ethoxy groups. The primary reaction is then described by the following equation;

$$
-\frac{d[T E S P T]}{d t}=k_{a}[T E S P T]=\frac{1}{2} \frac{d[E t O H]}{d t}
$$

and

$$
\ln k_{a}=\ln A-\frac{E_{A}}{R T}
$$

where [TESPT] and $[\mathrm{EtOH}]$ are the TESPT and ethanol concentrations in $\mathrm{mol} / \mathrm{kg}$ compound, respectively, $t$ is time in minutes, $k_{a}$ is the reaction rate constant, $A$ is the Arrhenius factor, $E_{A}$ is the activation energy in $\mathrm{kJ} / \mathrm{mol}, R$ is the gas constant and $T$ is temperature in $\mathrm{K}$. From a plot of $\mathrm{In} \mathrm{k}_{\mathrm{a}}$ versus $1 / R T$, the activation energy of the primary reaction is derived to be equal to $47 \mathrm{~kJ} / \mathrm{mol}$.

Secondary reaction - There are three possible reaction paths in this step. However, the overall reaction rate constants are assumed to have similar values. Based on the study at 120 to $160^{\circ} \mathrm{C}$, the overall reactions can be described as follows.

$$
\frac{d[E t O H]}{d t}=2 k_{a}[T E S P T]+k_{b}[Z 1]+k_{b}[Z 2]+k_{b}[Z 3]
$$


and

$$
\ln k_{b}=\ln A-\frac{E_{A^{\prime}}}{R T}
$$

where [Z1], [Z2] and [Z3] are intermediate products concentrations in $\mathrm{mol} / \mathrm{kg}, E_{A^{\prime}}$, is the activation energy of the secondary reaction in $\mathrm{kJ} / \mathrm{mol}$.

From the plot of $\ln k_{b}$ against $1 / R T$, the activation energy of the secondary reaction was derived to be $28 \mathrm{~kJ} / \mathrm{mol}^{\text {[27] }}$

\subsubsection{Silane coupling agents}

Silane coupling agents are commonly used in the rubber industry to enhance the degree of reinforcement of silica. In the 1970s, silane coupling agents that contain sulfur atoms were introduced to improve the bonding between silica and rubber during the mixing and curing stages. TESPT was used in the "Green Tire" due to its bifunctional character. Ethoxy-groups of the silane react with silanol groups on the silica surface and the tetrasulfide group bonds with the rubber matrix during the curing stage.

Jesionowski and Krysztafkiewicz ${ }^{[31]}$ investigated the influence of silane coupling agents on the surface properties of precipitated silica. Three types of silane coupling agents; 3-mercaptopropyl trimethoxysilane, 3-aminopropyltriethoxysilane and vinyltris(2methoxyethoxy) silane, were studied. The results showed that increasing amounts of mercaptosilane used for the silica modification gradually decreased the tendency of silica to form agglomerates, but above five parts by weight of this silane, an excess of silane promoted adhesion between silica particles/aggregates and re-agglomeration again occurred. The use of aminosilane showed different results, as the tendency of silica agglomeration was increased due to hydrogen bonds between neighboring modified silica particles. Meanwhile, the use of vinylsilane to modify the silica surface could break down the agglomerate structure because vinyl groups are electrostatically inert and do not interact with other particles.

However, two disadvantages of mercaptosilane coupling agents were observed. These included an unpleasant odor during mixing especially at elevated temperatures, and reduction of the scorch time. Thus, this silane is not suitable to be used for products with long processing procedures. ${ }^{[8]}$ Bis(triethoxysilylpropyl)tetrasulfide (TESPT), the bifunctional silane, shows better scorch safety than 3-mercaptopropyl-trimethoxy silane. For a vulcanization temperature at $120^{\circ} \mathrm{C}$ and below, the increase in scorch time with an increase of TESPT content may be attributed to the longer chain of TESPT that consists of triethoxy groups on both ends of the structure. These bulky groups cause steric 
hindrance for the reaction. However, as the vulcanization temperature increases, the scorch time decreases with increasing TESPT concentration due to the fact that the steric hindrance of TESPT becomes less significant, ${ }^{[32]}$ and S-S bonds break down giving active sulfur for the crosslinking reaction. TESPT has a bifunctional structure with ethoxy-groups for reacting with hydroxyl groups on the silica surface during mixing, and tetrasulfide for crosslinking with rubber molecules during vulcanization. Therefore, TESPT also acts as a sulfur donor in which the sulfur-sulfur bonds in the tetrasulfide structure can easily be broken down under high shear and mixing temperature. The reactive sulfur then reacts with rubber molecules, leading to a decrease of scorch time and increase of compound viscosity. The lower sulfur rank in a sulfur-containing silane such as Bis(triethoxysilylpropyl)disulfide (TESPD) provides better scorch safety compared to TESPT, but shows lower tensile modulus of the vulcanizates when compared to the ones with TESPT. ${ }^{[33]}$

In the case of silica-filled Nitrile-butadiene rubber (NBR), the effect of curing systems and silane coupling agent types at a fixed content of 2 phr were studied. It was found that the addition of 3-thiocyanatopropyl triethoxysilane in a silica-filled compound could reduce the filler-filler interaction to a greater extent than TESPT, due to its less bulky structure and lower viscosity leading to improved compound processability and enhanced mechanical properties of the rubber vulcanizates. For conventional vulcanization (CV) and semi-efficient vulcanization (semi-EV), 3-thiocyanatopropyl triethoxysilane gave a higher degree of silica reinforcement than TESPT, attributed to the effect of better filler dispersion and stronger filler-rubber interaction. However, for efficient vulcanization (EV), TESPT yielded a greater degree of silica reinforcement than 3-thiocyanatopropyl triethoxysilane due to the sulfur contribution effect when a large amount of accelerators was used. ${ }^{[34]}$ The optimum loading levels of TESPT and 3-thiocyanatopropyl triethoxysilane in the EV system were at 3.0 and $1.5 \mathrm{phr}$, respectively. The silica-filled compounds with 3thiocyanatopropyl triethoxysilane showed better compound processability than the silicafilled compounds with TESPT, but TEPST exhibited greater cure characteristics due to its ability to contribute sulfur during the vulcanization process. TESPT-containing vulcanizates showed better dynamic properties than 3-thiocyanatopropyl triethoxysilane, but poorer aging resistance. ${ }^{[35]}$

Due to some drawbacks of the 3-mercaptosilane and TESPT coupling agents as mentioned above, a new silane type was invented. A mercaptosilane blocked with an isocyanate-functional group in solid form was introduced to be used as a silane coupling agent for tires. The use of this isocyanate-blocked mercaptosilane in a silica-filled compound could avoid sulfur smell during mixing, but no improvement in processability 
was observed. Moreover, there was some toxicity released from the isocyanate during rubber processing. ${ }^{[36]}$

In recent years, mercaptosilane-derivatives with blocking groups such as a carboxylate ester or carboxylic acid functional groups are proposed. The use of these blocked mercaptosilanes in silica-filled rubber compounds needs a deblocking agent to obtain the optimum properties of the compound. ${ }^{[37,38]}$ The deblocking agent is a material that is capable to unblock the mercaptosilane to enable the mercapto-group to react with rubber molecules. The suitability of the deblocking agent depends on the blocking group which was used to block the reactivity of the mercaptosilane. The quantities of deblocking agent used are in the range of 0.1 to $5.0 \mathrm{phr}$, preferably in the range of 0.5 to $3.0 \mathrm{phr}$. Otherwise, the deblocking agent should be a nucleophilic molecule containing a hydrogen atom which can easily transfer to the blocking group in the blocked mercaptosilane, such as primary, secondary and tertiary amines, Lewis acids, or thiols. ${ }^{[39,40]}$ Other examples of deblocking agents are accelerators that are commonly used in sulfur cure systems such as thiuram, thiazole and sulphenamide. ${ }^{[41]}$ The addition of blocked mercaptosilane improves the properties of silica-filled rubber compounds, i.e. increased reinforcing efficiency, lowered compound viscosity and enhanced processability. The new silanes are designed to be used for several rubber products, e.g. tire treads, transmission belts, conveyor belts, roller coating, damping elements, etc. ${ }^{[42]}$

3-Octanoylthio-1-propyltriethoxysilane or NXT-silane is an octanoyl-group blocked mercaptosilane which provides a lower reactivity of the silane during processing. Silica-filled rubber compounds with NXT-silane should be prepared at high mixing temperature above $130^{\circ} \mathrm{C}$ to promote the reactions between the silane and silica. This blocked silane shows good scorch safety during mixing, even if the silica-NXT-filled compound is mixed for $10 \mathrm{~min}$ at $150^{\circ} \mathrm{C}$ to ensure a complete reaction between silica and silane. ${ }^{[43]}$ The hardness, modulus, tensile and tear strength are increased when the NXTsilane is present in the silica-filled compound. Furthermore, the wet traction and rolling resistance are improved by incorporating of the NXT-silane due to an increase of crosslink density in the compound. ${ }^{[4]}$ The scorch time of a silica-filled compound with NXT is longer than that of one with TESPT. The activation energies of the vulcanization reaction decrease with increasing NXT and TESPT concentrations in the silica-filled compounds, but the values of the compounds with NXT are lower than those of the ones with TESPT. This is due to steric hindrance of TESPT bulky end groups that retard the curing process. ${ }^{[45]}$ The silica particles modified with NXT-silane have a lower capacity to form aggregates and agglomerates. However, the only one sulfur atom in the NXT molecule is not able to form as strong an interaction and bonding to rubber molecules like the case of 
the tetrasulfide in TESPT, leading to lower mechanical properties of the silica/NXT-filled compound. ${ }^{[46]}$

\subsubsection{Flocculation of silica}

The re-agglomeration of filler aggregates or the reformation of a filler network, so called filler-flocculation, has an influence on the final properties of the filled-compounds. When the polarity of rubber is increased, polar fillers exhibit a decrease of flocculation tendency, whereas non-polar fillers tend to provide more flocculation. For example, it has been reported that EPDM filled with methylated fumed silica showed a lower flocculation tendency when compared to EPDM filled with unmodified silica. ${ }^{[47]}$ The flocculation process of the silica-silica network in the silica-filled compounds strongly increased during heat treatment. This phenomenon could also be observed after silanization but to a lesser degree due to the shielding of the silica surface. ${ }^{[26]}$

The degree of silica distribution or dispersion, $x$, can be expressed by the ratio of the Payne effect at time, $t$ and at infinite time; ${ }^{[30]}$

$$
x=\frac{s^{\prime}(t)-s^{\prime}(1)}{s^{\prime}(\infty)-s^{\prime}(1)}
$$

where $s^{\prime}(1), s^{\prime}(t)$ and $s^{\prime}(\infty)$ are the in-phase part of the dynamic torques measured in a Rubber Process Analyzer (RPA 2000, Alpha Technologies) after preheating time for 1 minute and after heating times for $t$ and 12 minutes, respectively. Herein, heating time at 12 minutes is taken as infinite time for practical purposes. Thus, the silica flocculation can be described by a simple kinetic parameter through the reaction rate constant, $k_{a}$ and the activation energy, $E_{a}$, as follows;

$$
\begin{aligned}
& k_{a}=\frac{\ln \left(1-x_{1}\right)-\ln \left(1-x_{2}\right)}{t_{2}-t_{1}}\left[\min ^{-1}\right] \\
& \ln k_{a} \approx-\frac{E_{a}}{R T}[\mathrm{~kJ} / \mathrm{mol}]
\end{aligned}
$$

where $t$ is the heating time, $x_{1}$ and $x_{2}$ are the degrees of silica dispersion at times $t_{1}$ and $t_{2}$, respectively, $T$ is the absolute temperature and $\mathrm{R}$ is the gas constant. The 
activation energy of flocculation can be obtained from the slope of the straight line of a plot of $k_{a}$ on a logarithmic scale against the inverse temperature.
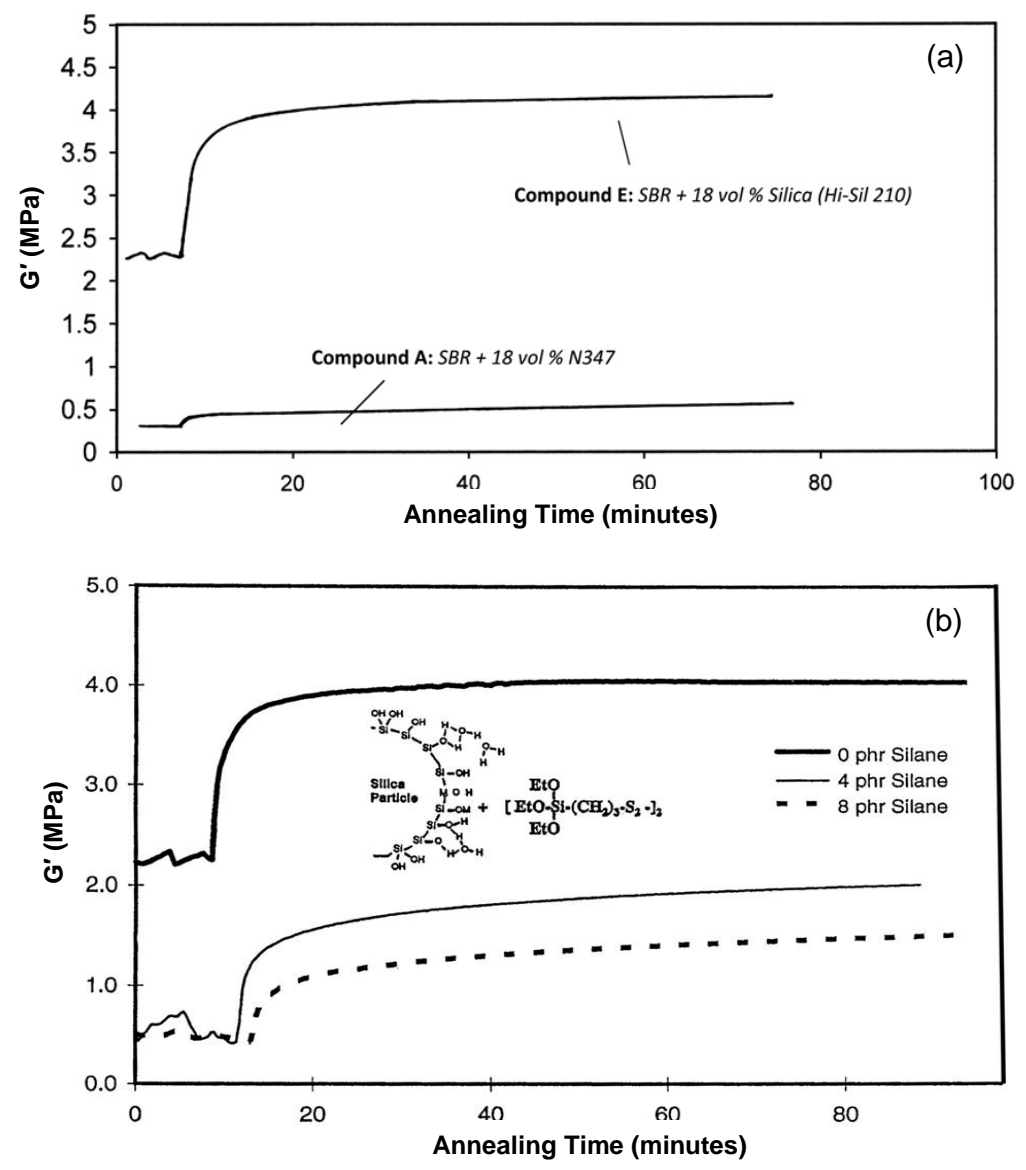

Figure 2.8 Shear storage modulus $\left(G^{\prime}\right)$ at $1 \%$ cyclic strain of filled-compounds: (a) comparison of silica and carbon black-filled compounds, and (b) silica-filled compounds with various silane loadings. ${ }^{[48]}$

Böhm et al. ${ }^{[48]}$ studied the flocculation process in carbon black and silica-filled compounds after annealing at $125-170^{\circ} \mathrm{C}$. As shown in Figure 2.8, the storage modulus $\left(G^{\prime}\right)$ of the compounds increased due to filler flocculation, and silica shows a much stronger filler flocculation than carbon black due to strong hydrogen bonding with other particles/aggregates. However, silica flocculation can be decreased by using a silane coupling agent, as observed in Figure 2.8(b). Lin et al. ${ }^{[49]}$ reported that the degree of silica flocculation depends on both type and concentration of the silane used in the compounds. 
Alkyl mono-(triethoxy silane) such as n-octyltriethoxysilane (OTES) and 3-mercaptopropyl triethoxysilane (MPS) could reduce silica flocculation during thermal annealing more effectively, when compared to bis-(trialkoxysilanes) such as bis-(triethoxysilylpropyl) tetrasulfide (TESPT), bis-(triethoxysilylpropyl)disulfide TESPD and bis(triethoxysilyl)octane (TESO). On the other side, silica dispersing agents such as sorbitan monooleate (SMO) and $\mathrm{N}, \mathrm{N}$-dimethyl-1-octadecylamine (DMOD) were not effective to prevent flocculation in the silica-filled compounds.

\subsubsection{Other approaches towards better dispersion of silica in a rubber matrix}

\subsubsection{1) In situ silica}

Recently, in situ silica synthesized by a sol-gel method has been widely studied as an alternative method to improve silica dispersion in a rubber matrix, in addition to a more common dry mixing or compounding method. This process consists of hydrolysis and condensation reactions of an alkoxysilane such as tetraethoxysilane (TEOS). The in situ silica generated is expected to be well-dispersed within the polymer matrix. The structure and morphology of the filler depend on the reaction conditions and nature of the catalyst. The in situ silica synthesis can be carried out in two different ways for the swollen state of a rubber matrix; either 1) the previously cross-linked polymer is swollen in TEOS and then hydrolyzed in situ, or 2) silica particles are precipitated in the polymer matrix before the cross-linking process. ${ }^{[50]}$ In addition to the swollen state, the in situ technique can be carried out in solution ${ }^{[51]}$ and in the latex states. ${ }^{[52,53]}$

Various factors affect the silica content and silica particle sizes obtained from the sol-gel method, such as the silica precursor and catalyst. Tetraethoxysilane (TEOS) has been used as silica precursor in the in situ silica reaction. The proportion of TEOS and water for the hydrolysis reaction was found to have an influence on the silica content obtained in the system. Increasing the TEOS/water molar ratio resulted in increasing of the silica content in the matrix. ${ }^{[54]}$ Other types of alkoxysilanes have been also applied to produce in situ silica such as vinylethoxysilane (VTOS), ethyltriethoxysilane (ETOS), and $i$-butyltriethoxysilane (BTOS). The results have shown that TEOS and VTOS are the most promising silica precursors. It was demonstrated that in situ generated silica can enhance the tensile modulus and tear resistance of rubber vulcanizates. ${ }^{[55]}$

Ikeda et al. ${ }^{[56]}$ investigated the effect of hydrochloric acid and n-butylamine as catalysts for the in situ silica process in a SBR matrix. The results show that the silica formed by the reaction with n-butylamine was homogeneously dispersed in the SBR matrix and the diameter of the particles was ca. $25-30 \mathrm{~nm}$. The primary alkylamines with 
long hydrocarbon segments were estimated to form reverse micelles like surfactant in the TEOS-swollen NR matrix ${ }^{[57]}$ as shown in Figure 2.10. Other primary alkylamines with different hydrocarbon segments, i.e., n-butylamine, n-hexylamine, n-octylamine, dipropylamine, and triethylamine were also investigated as catalysts for preparation of the in situ silica. The $n$-hexylamine catalyst showed a higher in situ silica content and good silica dispersion than the other catalysts. In addition, the amount of in situ silica increased with increasing $n$-hexylamine content. This is due to the fact that $n$-hexylamine contains a long chain alkyl group and its solubility in water is higher than that of $n$-octylamine. Thus, it can easily penetrate into the NR matrix and account for a more homogeneous reaction system. ${ }^{[58]}$ For the latex state reaction, an ammonia solution was used as catalyst. ${ }^{[50]}$
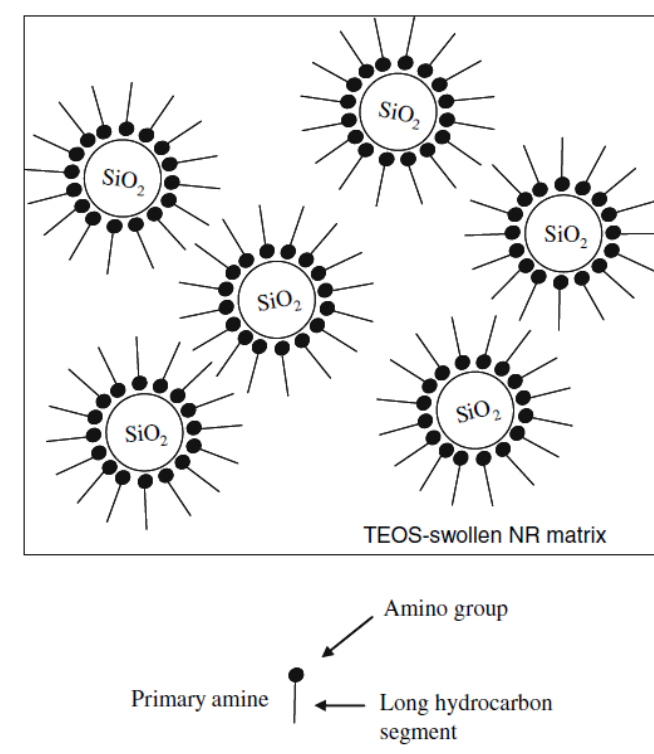

Figure 2.10 Speculated formation of in situ silica in a TEOS-swollen NR-matrix by primary amine with a long hydrocarbon segment. ${ }^{[58]}$

\subsubsection{2) Self-assembly process}

A novel self-assembly nanocomposite was developed to prepare a bulk poly(vinyl alcohol)/silica nanocomposite. ${ }^{[59]}$ It was found that the chemical and physical properties of such nanocomposite were significantly increased, due to the uniform distribution of silica particles and strong interaction between silica and polymer matrix. This novel process has also been applied to prepare NR/silica nanocomposites by combining the latex compounding and self-assembly technique. First, negatively charged silica nanoparticles act as templates to adsorb positively charged poly(diallyldimethylammonium chloride) 
(PDDA) molecules through electrostatic adsorption. Negatively charged natural rubber latex (NRL) particles are then assembled onto the surface of the silica/PDDA nanoparticles. Finally, the silica nanoparticles are uniformly distributed in the NR matrix, as shown in Figure 2.11. The key procedure of this process is the encapsulation of the silica nanoparicles with PDDA molecules and NRL matrix. The aim of the self-assembly process is to suppress the silica self-agglomeration reaction caused by strong silica-silica interaction. ${ }^{[60]}$ When the nanosilica content is less than $4.0 \mathrm{wt} \%$, the silica nanoparticles are assembled within NRL matrix as core-shell structure and the average size of the nanosilica clusters range between $65-80 \mathrm{~nm}$. It has been reported that the mechanical properties of the nanocomposites were markedly improved with increasing nanosilica loadings from 2.5 to $4.0 \mathrm{wt} \%$. Thermal and thermo-oxidative decomposition temperatures of the nanocomposites were also increased upon increasing silica loadings. ${ }^{[61]}$

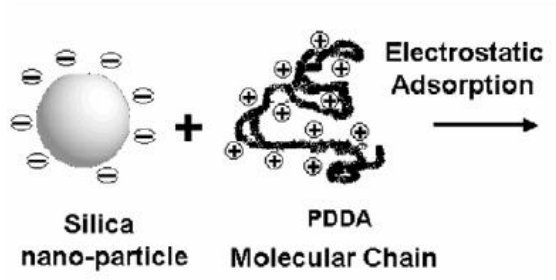

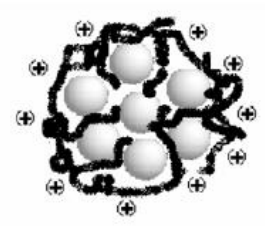

Electrostatic Adsorption

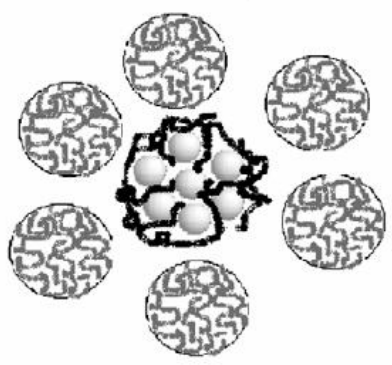
Nanocomposite

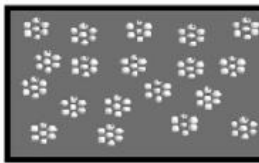

$\mathrm{NR} / \mathrm{SiO}_{2}$

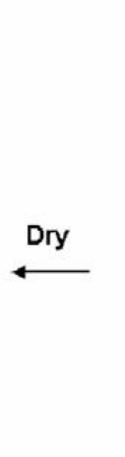

Figure 2.11 Scheme of the self-assembly process. ${ }^{[61]}$

\subsection{Compatibilizers for silica-reinforced rubbers}

Silica shows a high polarity and hydrophilic surface due to the silanol groups on its surface. Thus, it is less compatible with non-polar rubbers such as natural rubber (NR), styrene-butadiene rubber (SBR) and butadiene rubber (BR), and more compatible with polar rubbers such as chloroprene rubber (CR) and acrylonitrile butadiene rubber (NBR). 
The polar functional groups on the silica surface form hydrogen bonds with polar components in the rubber compound such as N-tert-butyl-2-benzothiazole sulfenamide (TBBS) and N-cyclohexyl benzothiazole sulfenamide (CBS) which are generally used as vulcanization accelerators for rubber compounds. These accelerators have alkaline functional groups in their structures, so they can be easily adsorbed on the acidic surface of silica. Even though silane coupling agents have been widely used to improve silica dispersion and to prevent adsorption of polar chemicals on the silica surface, the influence of polar rubber as an alternative to silane for the silica-filled compounds has also been studied.

Epoxidized natural rubber (ENR) shows a higher polarity than NR due to the epoxide groups in its structure. The chemical and physical properties of ENR change according to the epoxide group contents. The increase of epoxide contents decreases double bonds in the NR molecule leading to an increase of heat resistance and swelling resistance in non-polar solvents. The viscosity is also increased when the degree of epoxidation is increased. ${ }^{[62]}$ From the effect of polar functional groups, ENR interacts with hydroxyl groups on the silica surfaces. It has been reported that the mechanical properties of silica-filled ENR without silane coupling agent were higher than those of silica-filled NR without silane coupling agent. This was due to the improved interaction between the ENR and silica surface via hydrogen bonds. ${ }^{[63]}$ Reinforcing efficiency of silica-filled ENR was significantly improved by the good combination of ENR with the range of epoxide groups of $20-30 \mathrm{~mol} \%$ and TESPT in the range of $2-4 \mathrm{wt} \%$ relative to silica content. ${ }^{[64]}$ The silicafilled ENR in a presence of $N$-3-( $N$-vinyl-benzyl-amino)ethyl-y-amino-propyltrimethoxysilane-monochloride as a silane coupling agent led to a reaction between the $\mathrm{NH}_{2}{ }^{+}$- groups of the silane and epoxide groups of ENR resulting in a covalent $\mathrm{C}-\mathrm{N}$ bond between the silane and ENR, as shown in Figure 2.12. The extent of interaction between silica and ENR increased with increasing the volume fraction of filler and silane loading. ${ }^{[65]}$ Furthermore, the addition of a multifunctional additive (MFA) which is a diamine salt of a fatty acid $\left(\left[\mathrm{RNH}_{2}{ }^{+}\left(\mathrm{CH}_{2}\right)_{3} \mathrm{NH}_{3}{ }^{+}\right]\left[\mathrm{R}^{\prime} \mathrm{COO}_{2}\right]_{2}\right)$ could improve the mechanical properties of the silica-filled ENR. Scorch and cure times were decreased with increasing loading level of MFA. ${ }^{[66]}$ 
(a)

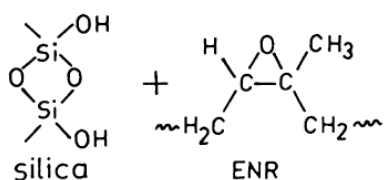

silica

ENR

Extrusion at $160^{\circ} \mathrm{C}$

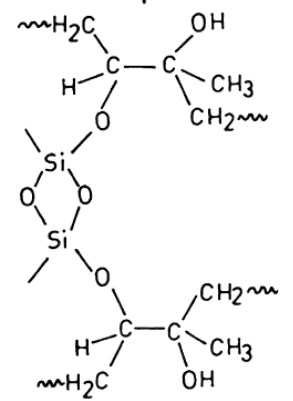

(b)

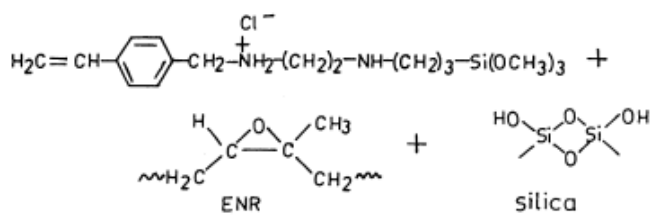

Extrusion at $160^{\circ} \mathrm{C}$

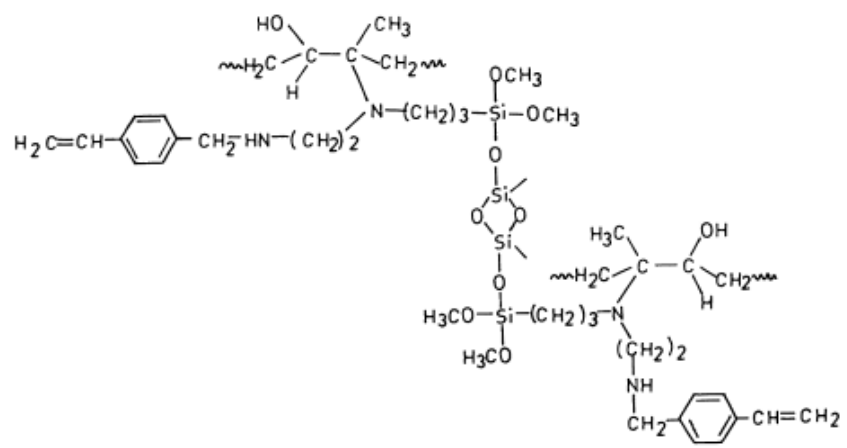

Figure 2.12 Proposed mechanism of the reaction between ENR and silica without silane (a) and in presence of $N$-3-( $N$-vinyl-benzyl-amino)ethyl-y-amino-propyl-trimethoxysilanemonochloride (b). ${ }^{[66]}$

George et al. ${ }^{[67]}$ investigated the addition of ENR as a compatibilizer in silica-filled NBR vulcanizates. Incorporation of ENR in silica-filled NBR showed improved properties of the vulcanizates. It was indicated that NBR and ENR can form self-crosslinked 
structures and the epoxy groups of ENR could react with hydroxyl groups on the silica surface, as shown in Figure 2.13, leading to an increase of bound rubber and mechanical properties. The properties of silica-filled NBR with ENR as a compatibilizer were higher than those of silica-filled NBR without ENR, and similar to those of silica-filled NBR with TESPT. The overall property enhancement indicated that ENR could be used as a reinforcement modifier in silica-filled NBR.

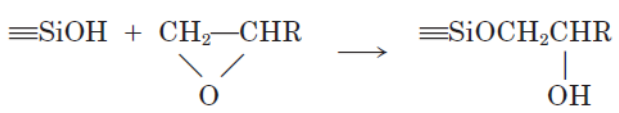

Figure 2.13 Proposed mechanism of reaction between ENR and silica. ${ }^{[67]}$

The effect of ENRs with 25 mol\% (ENR-25) and 50 mol\% epoxidation (ENR-50) as compatibilizers in NR/organoclay nanocomposites was studied. ${ }^{[68]}$ The results showed that dispersion of organoclay in the matrix, tensile strength and tear strength of ENR-50 containing nanocomposites were superior to those of ENR-25 compatibilized and uncompatibilized nanocomposites, respectively. Due to the ENR polar character, a higher interaction with organoclay was observed, leading to the improvement of dispersion of the organoclay in the rubber matrix. ${ }^{[69]}$ ENR/organoclay nanocomposites were used to improve the properties of carbon black filled-NR/high styrene rubber blends. ${ }^{[70]}$ The blends containing the nanocomposites at $10 \mathrm{phr}$ showed high crosslink density, maximum cure torque and cure rate index. The overall mechanical and thermal properties were improved by adding the ENR/organoclay in the blends. The interaction between ENR and clay that was formed during high-temperature $\left(180^{\circ} \mathrm{C}\right)$ molding could be observed by the infrared spectroscopic technique. ${ }^{[71]} \mathrm{A}$ decrease of peak intensities at 1568, 1348 and $3696 \mathrm{~cm}^{-1}$, which were assigned to $\mathrm{O}-\mathrm{H}$ bending of $\mathrm{H}-\mathrm{O}-\mathrm{H}$ and $\mathrm{Si}-\mathrm{OH}$, and $\mathrm{O}-\mathrm{H}$ stretching of $\mathrm{Si}-\mathrm{OH}$, respectively, as well as a decrease of the peak intensities at 870 and $840 \mathrm{~cm}^{-1}$ assigned to characteristic peaks of the epoxy group, indicated strong interaction between ENR and clay. The possible reaction is shown in Figure 2.14. 


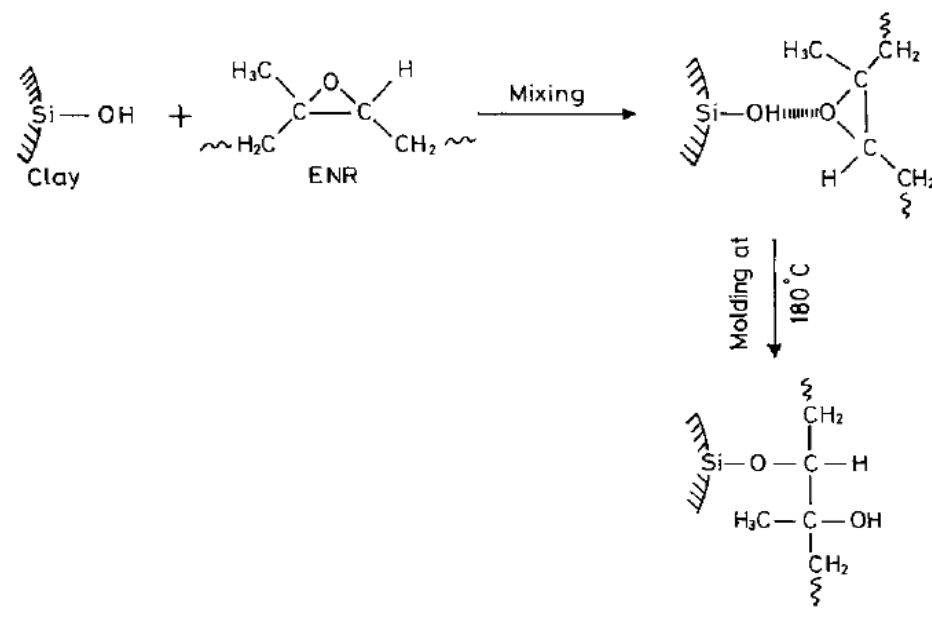

Figure 2.14 Proposed mechanism of bonding between ENR and clay. ${ }^{[71]}$

Maleic anhydride is another polar substance which can potentially react with hydroxyl groups on the silica surface so that silica agglomerates can be deminished or agglomerate size is reduced. Bikiaris and coworkers $^{[72]}$ studied the effect of poly(propylene-g-maleic anhydride) copolymer (PP-g-MA) as a compatibilizer in isotactic polypropylene (iPP)/ silica nanocomposites. PP-g-MA containing 0.6 wt\% of maleic anhydride content was added to the nanocomposites at various concentrations, i.e. 1, 2.5 and $5 \mathrm{wt} \%$ relative to the silica content. The tensile strength, elongation at break and modulus were increased with increasing nanosilica content as well as the copolymer content due to the enhancement of interfacial adhesion between PP and silica.

Maleated natural rubber (MNR) is a modified natural rubber prepared by grafting of maleic anhydride onto natural rubber molecules so that the MNR shows higher polarity than NR and better compatibility with silica. The interaction between MNR and silica is shown in Figure 2.15. The silica-silica interaction of silica-filled MNR was decreased as the succinic anhydride groups of the MNR could interact with the hydroxyl groups on the silica surface. The silica-filled MNR compound which was obtained from modified NR with $6 w t \%$ of maleic anhydride relative to the NR showed the lowest silica-silica interaction and optimum mechanical properties. The addition of a silane coupling agent in the compound did further increase silica-rubber interaction and decrease the silica-silica bonding, leading to improved mechanical and dynamic properties. ${ }^{[73]} \mathrm{A}$ study based on sludge paper which has hydroxyl groups on its surface similar to that of silica surfaces also found that the addition of MNR at $5 \mathrm{phr}$ as a compatibilizer into a sludge paper-filled NR composite improved the mechanical properties and enhanced adhesion between 
rubber and sludge. ${ }^{[74]}$ In addition, fatigue life of the composites was improved, while filler agglomerates were decreased. ${ }^{[75]}$

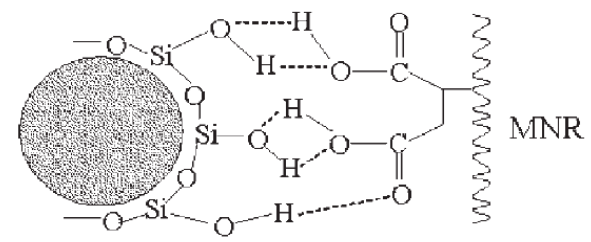

(a)

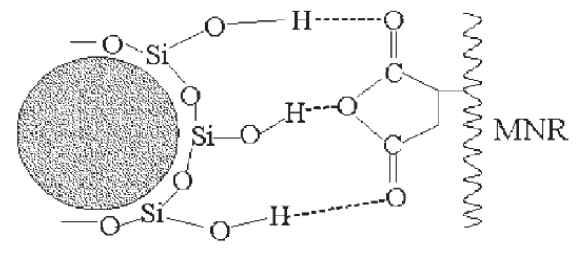

(b)

Figure 2.15 Postulated interaction between the maleic anhydride groups of MNR and the hydroxyl groups on the silica surface. ${ }^{[73]}$

Acrylonitrile butadiene rubber (NBR) is a polar rubber containing basic nitrile groups (-CN) which can form hydrogen bonds with silanol groups on the silica surfaces. Therefore, bound rubber between silica and NBR can be easily generated through hydrogen bonding in the silica-filled NBR. ${ }^{[76]}$

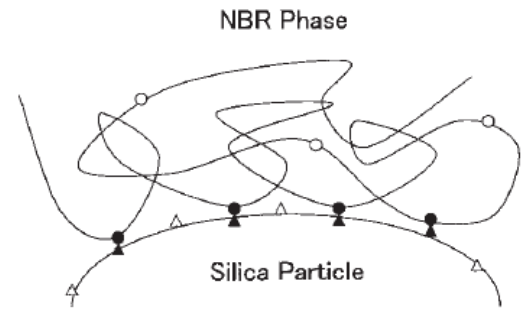

Low Nitrile group Content

Figure 2.16 Propose of bound rubber between NBR and silica surface. ${ }^{[76]}$

NBR has been claimed to act as a compatibilizer in silica-filled non-polar rubbers. The addition of NBR into a silica-filled styrene-butadiene rubber (SBR) compound improved the silica dispersion and physical properties of the vulcanizates whereas the scorch and cure times of the compounds decreased. ${ }^{[77]}$ The properties of the vulcanizates increased with increasing the acrylonitrile content of NBR. The optimum NBR content was at $5 \mathrm{phr}$ where the compound showed low viscosity and the vulcanizates had higher elongation at break, tensile strength and modulus. The addition of the silane coupling agent, Bis-(3-triethoxysilyl-propyl)tetrasulfide (TESPT) together with NBR into the silica- 
filled SBR compound further improved compatability between the silica and rubber phases. ${ }^{[78]}$ The effect of NBR on the properties of silica-filled NR was studied in the presence of the silane coupling agent, 3-octanoylthio-1-propyltriethoxysilane (NXT). The mechanical properties of the vulcanizates increased with increasing NBR and silane coupling agent contents. However, the addition of NBR more than 5 phr led to a continuous decrease of the mechanical properties of the vulcanizates. ${ }^{[79]}$

Polychloroprene rubber $(\mathrm{CR})$ is widely used to produce rubber products with requirements for oxidation, oil and heat resistance. $\mathrm{CR}$ is a homopolymer consisting of chloroprene units in which chlorine atoms induce its high polarity. ${ }^{[80]} \mathrm{CR}$ could be selfcrosslinked within a silica compound through reaction between allylic chlorine atoms and silanol groups. ${ }^{[81]}$ Similar to the silica-filled non-polar rubbers, properties of silica-filled CR can be improved by the addition of a silane coupling agent. The use of Bis-(3triethoxysilylpropyl)tetrasulfide (TESPT) showed better property improvement of filled-CR, compared to 3-thiocyanatopropyl triethoxy silane (Si-264). ${ }^{\left[{ }^{[2]}\right.}$ Metal oxide (ZnO) vulcanized silica-filled CR in presence of ethylene thiourea (ETU) showed an increase of crosslink density with increasing ETU loading levels, due to the nitrogen atom in ETU which could interact with silanol groups on the silica surface and form hydrogen bonds. The results also showed an increase of crosslink density with increasing silica loading levels because of a chemical reaction between allylic chlorine atoms and silanol groups. ${ }^{[83]}$
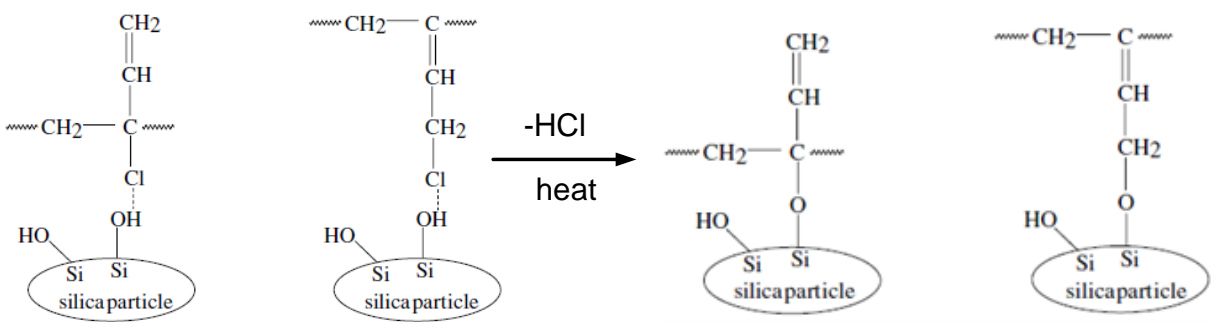

Figure 2.17 Probable reaction between the silanol groups and allylic chlorine atoms of $\mathrm{CR}^{[83]}$

The addition of CR into a silica-filled NR compound improved silica dispersion and increased bound rubber as well as physical properties such as tensile strength, elongation at break, modulus, abrasion and crack resistance. ${ }^{[84]}$ Increase of the CR content increased the properties of the filled-rubber, which could also be described by the fact that the electronegative chlorine atoms of CR might interact with hydroxyl groups on the silica surface to form hydrogen bonds, as shown in Figure $2.18,{ }^{[85]}$ instead of a full chemical reaction. ${ }^{[83]}$ 


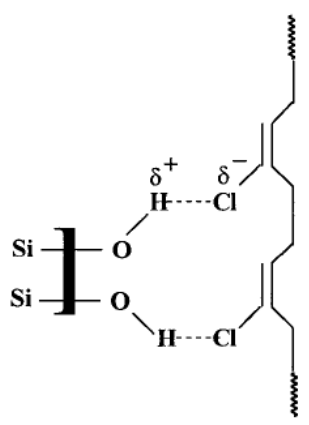

Figure 2.18 Interaction between chloroprene rubber and silica to form hydrogen bonds. ${ }^{[85]}$

Bertora et al. ${ }^{[86]}$ investigated the use of 3-mercaptopropyltrimethoxysilane (MPS)grafted polybutadiene oligomeric chains as compatibilizer in silica-filled SBR. The determination of the thermodynamic surface properties of the MPS-grafted polybutadiene modified silica showed a decrease of hydrophilic character of the silica. However, the silica-filled compound with the MPS-grafted polybutadiene gave lower mechanical properties than the silica-filled compound with TESPT.

\subsection{Silane modified polymers}

Silane-grafted polymers have been developed to improve the properties of some materials, for example, thermoplastic polyolefins, i.e. polyethylene (PE) and polypropylene (PP) and elastomers, e.g. ethylene-propylene rubber (EPM) and ethylene-propylene-diene terpolymer (EPDM). The silane-grafted polymers can be crosslinked in presence of moisture. Blends of polyolefins and silane-grafted polymers show advantages including improved extrudability of cable or tube jacket products, and high melting viscosity that allow accurate adjustment to specific needs. Also, strength, flexibility and electrical properties can be controlled through the level and proportion of the grafted polyolefins. ${ }^{[87]}$ The silane-grafted polyolefins are normally prepared in the melt in a twin-screw extruder $^{[88,89]}$ or internal mixer. ${ }^{[90]}$ The grafting efficiency of a vinyl-silane onto polymer molecules can be increased by using dicumyl peroxide (DCP) as initiator. ${ }^{[88]}$

Vinyloxyaminosilane grafted ethylene-propylene-diene terpolymer (EPDM- $g$ VOS) had been synthesized in toluene using DCP as initiator. ${ }^{[91]}$ The grafting efficiency increased with increasing EPDM content and reaction temperature when the grafting reaction was studied at $150-180^{\circ} \mathrm{C}$. The optimum VOS and DCP concentrations were 2 and 0.2 mole\%, respectively. By using the conditions at which the optimum grafting efficiency of VOS onto EPDM was obtained in the solution technique, the EPDM- $g$-VOS was also prepared in the melt at $160^{\circ} \mathrm{C}$ and rotor speed of $30 \mathrm{rpm}$ for $6 \mathrm{~min}$ in an internal 
mixer. The EPDM was mixed with 0.2 wt \% of DCP initiator dissolved in 2 wt $\%$ of VOS and 0.1 wt $\%$ of dibutyltindilaurate catalyst. The VOS-grafted EPDM showed low mechanical properties but its dielectric characteristics were improved with increasing VOS concentrations. The EPDM-g-VOS was later used to blend with linear low density polyethylene (LLDPE). Both phases were compatible at all blend compositions. The values of storage modulus $\left(E^{\prime}\right)$, loss modulus $\left(E^{\prime \prime}\right)$ and mechanical properties were increased with increase of LLDPE in the blends. ${ }^{\text {[92] }}$

Grafting of vinyltriethoxysilane (VTES) onto ethylene-propylene-diene terpolymer (EPDM) by using DCP as initiator was investigated by Alagar et al.. ${ }^{[93]}$ The EPDM- $g$-VTES was prepared by melt mixing in a twin-screw extruder. The effects of various parameters, i.e. EPDM and VTES contents, reaction time, reaction temperature and initiator concentration, on the grafting efficiency of VTES onto EPDM were investigated. The grafting efficiency linearly increased with increasing EPDM contents due to the fact that more active sites were present. The highest grafting efficiency of $18.7 \%$ was observed when the reaction was carried out using VTES at $5 \mathrm{wt} \%$. The grafting efficiency decreased with further increase of VTES concentration, due to the formation of more active centers of VTES which tended to result in homopolymerization rather than the grafting reaction. Optimized reaction temperature and reaction time were at $180^{\circ} \mathrm{C}$ and $5 \mathrm{~min}$, respectively. The grafting reaction of EPDM with VTES is shown in Figure 2.19.

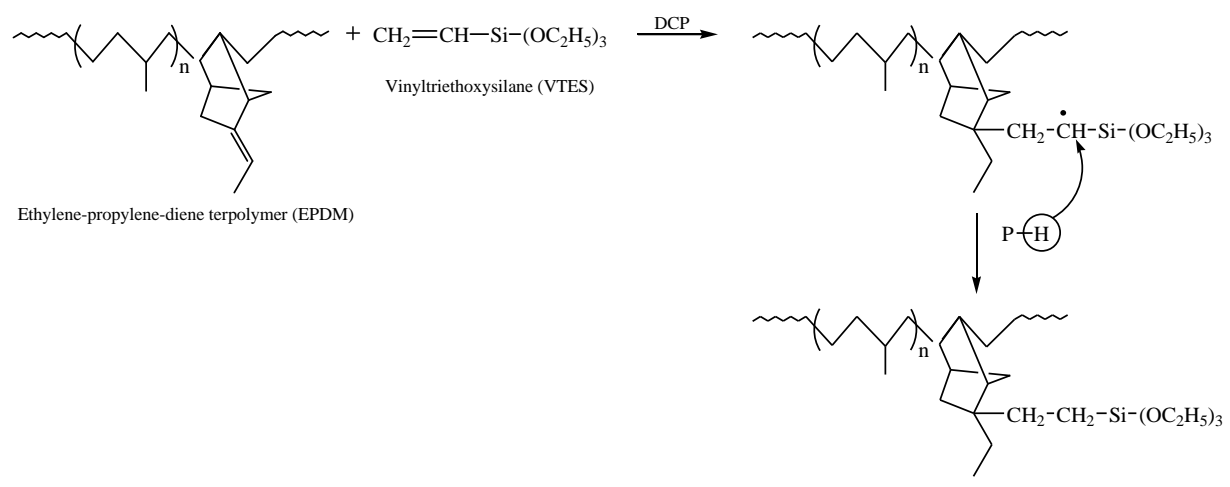

Figure 2.19 Schematic representation of the VTES-grafted EPDM. ${ }^{[93]}$

The EPDM- $g$-VTES was later blended with LLDPE. The mechanical properties such as tensile strength, modulus and hardness of EPDM- $g$-VTES/LLDPE blends were higher than those of EPDM/LLDPE blends due to the presence of efficient interaction between the blend components and the increase of crosslinking in EPDM- $g$-VTES. 
Changjie et al. ${ }^{[94]}$ recently studied the graft copolymerization of VTES onto SBR molecules in latex by using benzoic peroxide as initiator. The increase of VTES concentration reduced the grafting efficiency. The VTES-grafted SBR (SBR-g-VTES) vulcanizates showed an increase of tensile strength, thermal decomposition temperature and glass transition temperature, but a decrease of cure rate and elongation at break with increasing concentration of VTES.

3-Mercaptopropyltrimethoxysilane and 3-mercaptopropyltriethoxysilane were grafted onto a liquid low molar mass polybutadiene (PB) in solution state in order to obtain a product which is crosslinkable with moisture. The grafting reaction was carried out at $75^{\circ} \mathrm{C}$ for $3 \mathrm{~h}$ under nitrogen atmosphere and azobisisobutylonitrile (AIBN) was used as initiator. ${ }^{[95,96]}$ It was found that the triethoxysilane groups were attached onto the low molar mass polybutadiene in the range of 1.05 to 7.9 functions per chain. The reaction between 3-mercaptopropyltriethoxysilane (MPTS) and double bonds of polybutadiene is shown in Figure 2.20. The reaction occurred faster with double bonds in 1,2-polybutadiene than in 1,4-polybutadiene and the kinetics are very dependent on the chemical structure of the thiol.

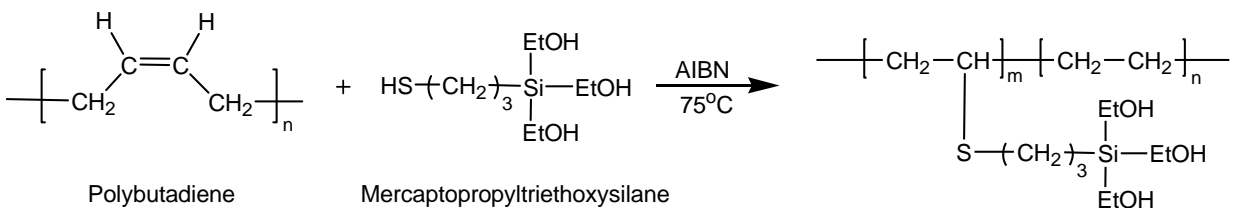

Figure 2.20 Mechanism of 3-mercaptopropyltriethoxysilane modification of polybutadiene. ${ }^{[96]}$

\subsection{Motivation of this thesis}

Silica has conventionally been used to produce light-colored products and mechanical goods, but is increasingly used in tire tread compounds to reduce rolling resistance and thus automotive fuel consumption. However, the large number of hydroxylgroups on the silica surface leads to strong filler-filler interactions and poor interaction with non-polar rubbers. Silane coupling agents have been practically used in silica-filled compounds to improve silica dispersion and interactions between silica and rubber. The mixing conditions must be optimized to achieve a good level of silanization and thus properties. The mixing of a silica-silane filled-compound is more complicated than that of carbon black-filled ones. A whole or partial replacement of silane coupling agent with chemically functionalized NR as a compatibilizer should reduce the complications arising 
from the silanization reaction. This will lead to use of more silica compounds for tires and consequently more efficient use of energy. A new market for modified NR can also be promoted. Silica is a natural oil-independent filler and NR is a renewable resource. The increasing use of silica and NR will drive the rubber industry towards greener technology.

In this thesis, modified natural rubbers are used as compatibilizers in silica-filled natural rubber compounds to achieve optimal properties of such compounds in comparison with the use of TESPT silane coupling agent. Natural rubber is first modified to achieve polar functional groups attached onto the natural rubber molecules, such as epoxide functional groups and silane coupling agent moieties. The silanes under investigation are vinyltriethoxysilane, 3-mercaptopropyltriethoxysilane and 3-octanoylthio1-propyltriethoxysilane (NXT). The modified natural rubbers are subsequently used as compatibilizers in silica-reinforced NR compounds, and their optimum loadings are investigated. The modified rubbers are also used in combination with silane coupling agents to enhance the properties of silica-filled natural rubber, by taking the compound with optimum TESPT silane coupling agent as reference. All of the silica-filled natural rubber compounds are characterized for their Mooney viscosities, cure behavior, filler-filler interaction or Payne effect, filler-rubber interaction by means of bound rubber measurements, mechanical properties, dynamic mechanical properties and silica dispersion.

\subsection{References}

1. B. Schwaiger, A. Blume, Rubber World, 4, 32 (2000).

2. R. Rauline, EP Patent 0 501227A1, to Michelin \& Cie, February 9, 1992.

3. J.L. Leblanc, Prog. Polym. Sci., 27, 627 (2002).

4. M. Morton, "Rubber Technology", Robert E. Krieger Publishing, Florida, second edition, 1981.

5. C.M. Blow, C. Hepburn, "Rubber Technology and Manufacture", Butterworths, London, second edition, 1982.

6. J. Fröhlich, W. Niedermeier, H.-D. Luginsland, Composites: Part A, 36, 449 (2005).

7. R.W. Magee, Rubber Chem. Technol., 68, 590 (1995).

8. S. Wolff, Rubber Chem. Technol., 69, 325 (1996).

9. G. Kraus, "Science and Technology of Rubber", Academic Press, London, 1978.

10. A.I. Medalia, R.R. Juengel, J.M. Collins, Developments in Rubber Technology-1, Applied Science, London, 1979. 
11. W.W. Barbin, M.B. Rodgers, "Science and Technology of Rubber", Academic Press, San Diego, second edition, 1994.

12. L.T. Zhuravlev, Colloids Surf. A: Physicochem. Eng. Aspects, 173, 1 (2000).

13. M.P. Wagner, Rubber Chem. Technol., 49, 703 (1976).

14. M.J. Wang, S. Wolff, J.-B. Donnet, Rubber Chem. Technol., 64, 559 (1991).

15. M.J Wang, S. Wolff, Rubber Chem. Technol., 65, 715 (1992).

16. L.J. Murphy, M.-J. Wang, K. Mahmud, Rubber Chem. Technol., 71, 998 (1998).

17. L.J. Murphy, M.-J. Wang, K. Mahmud, Rubber Chem. Technol., 73, 25 (2000).

18. J.-H. Ma, S.-H. Zhao, L.Q. Zhang, Y.-P. Wu, Rubber Chem. Technol., 86, 664 (2013).

19. M.-J. Wang, H. Tu, L.J. Murphy, K. Mahmud, Rubber Chem. Technol., 73, 666 (2000).

20. A.R. Payne, Rubber Chem. Technol., 44, 440 (1971).

21. L.J. Murphy, E. Khmelnitskaia, M.J. Wang, K. Mahmud, Rubber Chem. Technol., 71, 1015 (1998).

22. M.-J. Wang, P. Zhang, K. Mahmud, Rubber Chem. Technol., 74, 124 (2001).

23. A. Einstein, Ann. der Physik, 19, 289 (1906).

24. J.-B. Donnet, Rubber Chem. Technol., 71, 323 (1998).

25. H.-D., Luginsland, J. Fröhlich, A. Wehmeier, Rubber Chem. Technol., 75, 563 (2002).

26. M.-J. Wang, Rubber Chem. Technol., 71, 521 (1998).

27. U. Görl, A. Hunsche, A. Mueller, H.G. Koban, Rubber Chem. Technol., 70, 608 (1997).

28. J.W.T. ten Brinke, S.C. Debnath, L.A.E.M. Reuvekamp, J.W.M. Noordermeer, Comp. Sci. Technol., 63, 1165 (2003).

29. W. Kaewsakul, K. Sahakaro, W.K. Dierkes, J.W.M. Noordermeer, Rubber Chem. Technol., 85, 277 (2012).

30. S.Mihara, R.N. Datta, J.W.M. Noordermeer, Rubber Chem. Technol., 82, 524 (2009).

31. T. Jesionowski, A. Krysztafkiewicz, Appl. Surf. Sci., 172, 18 (2001).

32. B.T. Poh, C.C. Ng, Eur. Polym. J., 34, 975 (1997).

33. K.-J. Kim, J. Vanderkooi, Kautsch. Gummi Kunstst., 55, 518 (2002).

34. P. Sae-oui, U. Thepsuwan, K. Hatthapanit, Polym. Test., 23, 397 (2004).

35. P. Sae-oui, C. Sirisinha, K. Hatthapanit, U. Thepsuwan, Polym. Test., 24, 439 (2005).

36. S. Ahmad, R.J. Schaefer, US Patent 4519430, to The B.F. Goodrich Company, May 28, 1985. 
37. R.W. Cruse, R.J. Pickwell, K.J. Weller, E.R. Pohl, US Patent 6528673 B2, to Crompton Corporation, March 4, 2003.

38. R.W. Cruse, R.J. Pickwell, K.J. Weller, E.R. Pohl, US Patent 6608125 B2, to Crompton Corporation, August 19, 2003.

39. M.P. Cohen, US Patent 2005/0009955 A1, to The GoodYear Tire \& Rubber Company, January 13, 2005.

40. R.W. Cruse, R.J. Pickwell, K.J. Weller, E.R. Pohl, US Patent 7122590 B2, to General Electric Company, October 17, 2006.

41. C.J. Lin, W.L. Hergenrother, US Patent 7256231 B2, to Bridgestone Corporation, August 14, 2007.

42. H.-D. Luginsland, R. Krafczyk, F. Forster, US Patent 2003/0130388 A1, to Degussa AG, July 10, 2003.

43. H. Yan, K. Sun, Y. Zhang, Y. Zhang, J. Appl. Polym. Sci., 94, 2295 (2004).

44. H. Yan, G. Tian, K. Sun, Y. Zhang, Y. Zhang, J. Appl. Sci. Part B: Polym. Phys., 43, 573 (2005).

45. H. Yan, K. Sun, Y. Zhang, Y. Zhang Y. Fan, J. Appl. Polym. Sci., 94, 1511 (2004).

46. J.L. Valentín, I. Mora-Barrontes, A. Rodríguez, L. Ibarra, L. Gonzalez, J. Appl. Polym. Sci., 103, 1806 (2007).

47. K. Stockelhuber, A. Das, R. Jurk, G. Heinrich, Polymer, 50, 1954 (2010).

48. G.A. Böhm, W. Tomaszewski, W. Cole, T. Hogan, Polymer, 51, 2057 (2010).

49. C.J. Lin, W.L. Hergenrother, E. Alexanian, G.G.A. Böhm, Rubber Chem. Technol., 75, 865 (2002).

50. L. Bokobza, J.-P. Chauvin, Polymer, 46, 4144 (2005).

51. B. Chaichua, P. Prasassarakich, S. Poompradub, J. Sol-Gel Sci. Technol., 52, 219 (2009).

52. K. Yoshikai, T. Ohsaki, M. Furukawa, J. Appl. Polym. Sci., 85, 2053 (2002).

53. V. Tangpasuthadol, A. Intasiri, D. Nuntivanich, N. Niyompanich, S. Kiatkamjornwong, J. Appl. Polym. Sci., 109, 424 (2008).

54. S. Poompradub, B. Chaichua, C. Kanchanaamporn, T. Boosalee, P. Prasassarakich, Kautsch. Gummi Kunstst., 4, 152 (2008).

55. J. Siramanont, V. Tangpasuthadol, A. Intasiri, N. Na-Ranong, S. Kiatkamjornwong, Polym. Eng. Sci., 49, 1099 (2009).

56. Y. Ikeda, A. Tanaka, A. Kohjiya, J. Mater. Chem., 7, 1497 (1997).

57. F. Debuigne, L. Jeunieau, M. Wiame, J.B. Nagy, Langmuir, 16, 7605 (2000).

58. Y. Ikeda, S. Poompradub, Y. Morita, S. Kohjiya, J. Sol-Gel Sci. Technol., 45, 299 (2008). 
59. Z. Peng, L.X. Kong, S.-D. Li, J. Appl. Polym. Sci., 96, 1436 (2005).

60. Z. Peng, L.X. Kong, S.-D. Li, J. Nanosci. Nanotechnol., 6, 541 (2006).

61. Z. Peng, L.X. Kong, S.-D. Li, Y. Chen, M.F. Huang, Comp. Sci. Technol., 67, 3130 (2007).

62. I.R. Gelling, M. Porter, "Natural Rubber Science and Technology", Oxford University Press, Oxford, 1988.

63. F. Cataldo, Macromol. Mater. Eng., 287, 348 (2002).

64. W. Kaewsakul, K. Sahakaro, W.K. Dierkes, J.W.M. Noordermeer, Adv. Mater. Res., 844, 243 (2014).

65. A.K. Manna, A.K. Bhattacharyya, P.P. De, D.K. Tripathy, S.K. De, D.G. Peiffer, Polymer, 39, 7113 (1998).

66. H. Ismail, H.H. Chia, Eur. Polym. J., 34, 1857 (1998).

67. K.M. George, J.K. Varkey, K.T. Thomas, N.M. Mathew, J. Appl. Polym. Sci., 85, 292 (2002).

68. P.L. Teh, Z.A.M. Ishak, A.S. Hashim, J. Karger-Kocsis, U.S. Ishiaku, Eur. Polym. J., 40, 2513 (2004).

69. M. Arroyo, M.A. Lopez-Manchado, J.L. Valentin, J. Carretero, Comp. Sci. Technol., 67, 1330 (2007).

70. K. Pale, R. Rajasekar, D.J. Kang, Z.X. Zhang, J.K. Kim, C.K. Das, Mater. Design., 30, 4035 (2009).

71. A.K. Manna, P.P. De, D.K. Tripathy, S.K. De, D.G. Peiffer, J. Appl. Polym. Sci., 74, 389 (1999).

72. D.N. Bikiaris, A. Vssiliou, E. Pavlidou, G.P. Karayannidis, Eur. Polym. J., 41, 1965 (2005).

73. K. Sahakaro, S. Beraheng, J. Appl. Polym. Sci., 109, 3839 (2008).

74. H. Ismail, A. Rusli, A.A. Rashid, Polym. Test., 24, 856 (2005).

75. H. Ismail, A. Rusli, A.R. Azura, J. Polym. Environ., 25, 67 (2007).

76. N. Suzuki, M.Ito, S. Ono, J. Appl. Polym. Sci., 95, 74 (2005).

77. S.-S. Choi, J. Appl. Polym. Sci., 79, 1127 (2001).

78. S.-S. Choi, J. Appl. Polym. Sci., 85, 385 (2002).

79. H. Yan, K. Sun, Y. Zhang, Y. Zhang, Polym. Test., 25, 32 (2005).

80. J.A. Brydson, "Rubber Chemistry”, Applied Science Publishers, London, 1978.

81. G. Wang, M. Li, X. Chen, J. Appl. Polym. Sci., 72, 577 (1999).

82. P. Sae-oui, C. Sirisinha, U. Thepsuwan, K. Hatthapanit, Eur. Polym. J., 42, 479 (2006). 
83. P. Sae-oui, C. Sirisinha, U. Thepsuwan, K. Hatthapanit, Eur. Polym. J., 43, 1853 (2007).

84. S.-S. Choi, J. Appl. Polym. Sci., 83, 2609 (2002).

85. A. Das, S.C. Debnath, D. De, D.K. Basu, J. Appl. Polym. Sci., 93, 196 (2004).

86. A. Bertora, M. Castellano, E. Marsano, M. Alessi, L. Conzatti, P. Stagnaro, Macromol. Mater. Eng., 296, 455 (2011).

87. H.-M. Schmidtchen, H.U. Voigt, US Patent 4618654, to Kabel-und Metallwerke Gutehoffnungshuette, October 21, 1986.

88. Y.-T. Shieh, C.-M. Liu, J. Appl. Polym. Sci., 74, 3404 (1999).

89. J.H. Shim, J.H. Joo, S.H.Jung, J.-S. Yoon, J. Polym. Sci. Part B: Polym. Phys., 45, 607 (2007).

90. J.S.Parent, R. Parodi, W. Wu, Polym. Eng. Sci., 46, 1754 (2006).

91. M.S.C. Kumar, M. Alagar, Eur. Polym. J., 38, 2023 (2002).

92. M.S.C. Kumar, M. Alagar, A.A. Pradu, Eur. Polym. J., 39, 805 (2003).

93. M. Alagar, S.M.A. Majeed, S. Nagendiran, Polym. Adv. Technol., 16, 582 (2005).

94. Y. Changjie, Z. Qiuyu, Y. Dezhong, G. Junwei, G. Guangbi, L. Tao, J. Appl. Polym. Sci., 119, 2808 (2011).

95. G. Colucci, A.D. Gianni, R. Bongiovanni, A. Priola, F. Torello, S. Bracco, P. Sozzani, Macromol. Symp., 296, 550 (2010).

96. F. Schapman, J.P. Couvercelle, C. Brunel, Polymer, 39, 4955 (1998). 
Chapter 2 


\section{Silica-Reinforced Tire Tread Compounds Compatibilized by Using Epoxidized Natural Rubber}

Epoxidized natural rubber (ENR) is a modified form of NR bearing polar epoxy groups. This chapter investigates a silica-reinforced NR tire tread compounds with ENR as a compatibilizer. The ENRs consisting of 10,38 and 51 mol\% epoxide are used in a range of 2.5 to 15.0 parts per hundred parts of rubber (phr). The addition of ENRs, especially ENR-38 and ENR-51, decreases the Mooney viscosity, Payne effect, flocculation rate constant and filler networking factor, which implies an improvement of silica dispersion in the compounds. Chemically bound rubber contents and interaction parameters of the compounds also increase with higher epoxide-contents of the ENRs, indicating more interactions and/or reaction between the epoxide-groups of the ENR and silanol groups on the silica surface. The different level of compatibility in the filled-NR vulcanizates that influences silica dispersion is also evidenced by SEM analysis. Tensile strength of the vulcanizates is improved with increasing mole\% epoxide, and the optimum value is observed at 7.5 phr of ENR-51. The presence of ENR as compatibilizer increases tan $\delta$ at both $0^{\circ} \mathrm{C}$ and $60^{\circ} \mathrm{C}$ which implies improved wet grip but also increased tire rolling resistance. The overall results show that silica-reinforced NR can be substantially improved by adding ENR as a compatibilizer, when compared to a compound without ENR, but somewhat less than with using a silane coupling agent. 


\subsection{Introduction}

Silica and carbon black are widely used reinforcing fillers in rubber. Because of their different surface characteristics, both fillers can form agglomerates but the cause of such agglomeration is not the same, leading to a difference in dispersability. The filler-filler interaction of carbon black is mainly generated through relatively weak Van der Waals forces, which can be readily broken during mixing. In contrast, silica agglomeration is due to hydrogen bonding in addition to Van der Waals forces and other physical interactions, leading to a much stronger filler-filler interaction. ${ }^{[1]}$ Silica has a high polarity and a hydrophilic surface due to silanol groups on its surface. Consequently, silica is incompatible with non-polar rubbers such as natural rubber (NR), styrene-butadiene rubber (SBR) and polybutadiene rubber $(\mathrm{BR})$, but better compatible with polar rubbers such as polychloroprene rubber $(\mathrm{CR})^{[2]}$ and acrylonitrile butadiene rubber (NBR) ${ }^{\left[{ }^{[3]}\right.}$ Use of silica in non-polar rubbers without compatibilizers results in poor dispersion and consequently inferior properties as a result of these strong filler-filler interactions. ${ }^{[4]}$ In addition, polar functional groups on the silica surface may form hydrogen bonds with other polar components in rubber compounds such as accelerators. So, commonly basic accelerators are adsorbed on the acidic surface of silica which negatively affects cure properties. $^{[5]}$ Successful use of silica for rubber reinforcement therefore requires commonly silane coupling agents to enhance silica-rubber interaction and silica dispersion as well as to prevent accelerator adsorption on the silica surface.

Some polar rubbers bearing functional groups which can interact with the silica surface have been studied as alternatives to silane coupling agents to enhance the interaction in silica-filled compounds. ${ }^{[6-9]} \mathrm{A}$ non polar rubber such as polybutadiene was chemically modified to have epoxy groups on the main chain and the interaction of such epoxidized rubber with silica was consequently improved, as observed by a decrease of the Payne effect ${ }^{[10]}$ and a change of damping behavior in the glass transition region. The improvement of silica-rubber interaction was also demonstrated by a higher bound rubber content and better silica dispersion in the matrix. ${ }^{[11]}$ Incorporation of silica into an epoxidized SBR showed a strong interaction between the silica and the epoxidized functional groups due to the highly polar character of the epoxidized rubber. ${ }^{[12]}$

Epoxidized natural rubber (ENR) is also higher polar than virgin NR due to the epoxide groups in its structure. The chemical and physical properties of ENR change according to the epoxide contents, such as heat and swelling resistances. ${ }^{[13]}$ By its polar functional groups, ENR interacts with hydroxyl groups on the silica surfaces. ${ }^{[14]}$ It has been reported already that the mechanical properties of silica-filled ENR without coupling agent 
are higher than those of silica-filled virgin $N R^{[15,16]}$ This was claimed to be due to improved interaction between ENR and the silica surface via hydrogen bonds. ENR as the rubber component in tire compounds has been reported to improve the silica dispersion leading to improved processability, better storage stability and tire performance. ${ }^{[14,17-19]}$ ENR was used as a compatibilizer in silica-filled NR/NBR blends to enhance the mechanical properties. ${ }^{[20]}$ In case of silica-filled NBR vulcanizates, the use of ENR as a compatibilizer improved tensile strength, tear strength and abrasion loss, ${ }^{[21]}$ as well as lowered the loss tangent, ${ }^{[22]}$ as the ENR acts as a coupling agent because it can self-crosslink with NBR and also link with silica at the vulcanization temperature. ${ }^{[21,22]}$ Furthermore, ENR was used as a compatibilizer in organoclay-filled NR composites wherein cure characteristics, tensile and dynamic mechanical properties were improved. ${ }^{[23,24]}$ Even though ENR has been used to compatibilize silica and various rubber matrices, the use of ENR as compatbilizer for silica-filled virgin NR has not yet been extensively studied.

In the present study, ENRs with different mole\% of epoxide groups are used as compatibilizers in a range of 2.5 to 15 phr for virgin NR/silica compounds. The effects of ENR contents and mole\% of epoxide are investigated. The epoxide groups are supposed to interact with the silanol groups of the silica and so to improve silica-rubber compatibilization, while the unmodified parts of the ENR structure are compatible with the NR matrix.

\subsection{Experimental}

\subsubsection{Materials}

The rubbers used were Natural Rubber (Ribbed Smoked Sheet (RSS) \#3), locally produced in Thailand, and Epoxidized Natural Rubbers with 10, 38 and $51 \mathrm{~mol} \%$ of epoxide, denoted as ENR-10, ENR-38 and ENR-51, respectively, that were in-house prepared as detailed in 3.2.2. The compounding ingredients were highly dispersible silica (Zeosil 1165MP, Rhodia, France), bis-(triethoxysilylpropyl)tetrasulfide (TESPT) (Evonik, Germany), treated distillate aromatic extract oil (TDAE oil) (Hansen \& Rosenthal, Germany), N-cyclohexyl-2-benzothiazole sulfenamide (CBS), diphenyl guanidine (DPG) and 2,2,4-trimethyl-1,2-dihydroquinoline (TMQ) (all from Flexys, Belgium), ZnO, stearic acid and sulfur (all from Sigma-Aldrich Chemie, Germany). 


\subsubsection{Preparation of epoxidized natural rubber}

High ammonia (HA) natural rubber latex with 60 wt\% of dry rubber content was used to prepare ENR via an in-situ performic epoxidation reaction. ${ }^{[14]}$ The reaction between $\mathrm{C}=\mathrm{C}$ of the NR molecule and performic acid, arising from a reaction between formic acid and hydrogen peroxide, was carried out in a continuously stirred reactor at a temperature of $40^{\circ} \mathrm{C}$, where alkylphenol ethoxylate non-ionic surfactant (Teric N30, Huntsman Corp., Australia) was used as a stabilizer. ${ }^{[25,26]}$ The ENRs with 10 , 38, and 51 mole\% of epoxide groups were obtained when the reaction times were varied at 2, 10 and $12 \mathrm{~h}$, respectively. The ENR latex was subsequently coagulated with methanol. The ENR coagulum was sheeted, washed thoroughly with water and dried in an oven at $50^{\circ} \mathrm{C}$ for approximately 4 days. The reaction time of epoxidation was set according to the required level of epoxide groups in the ENR product. The ${ }^{1} \mathrm{H}$ NMR spectroscopic technique was used to analyze the molecular structure of the ENR and a typical ${ }^{1} \mathrm{H}$ NMR spectrum of ENR is shown in Figure 3.1 with details of proton types assigned for each peak.

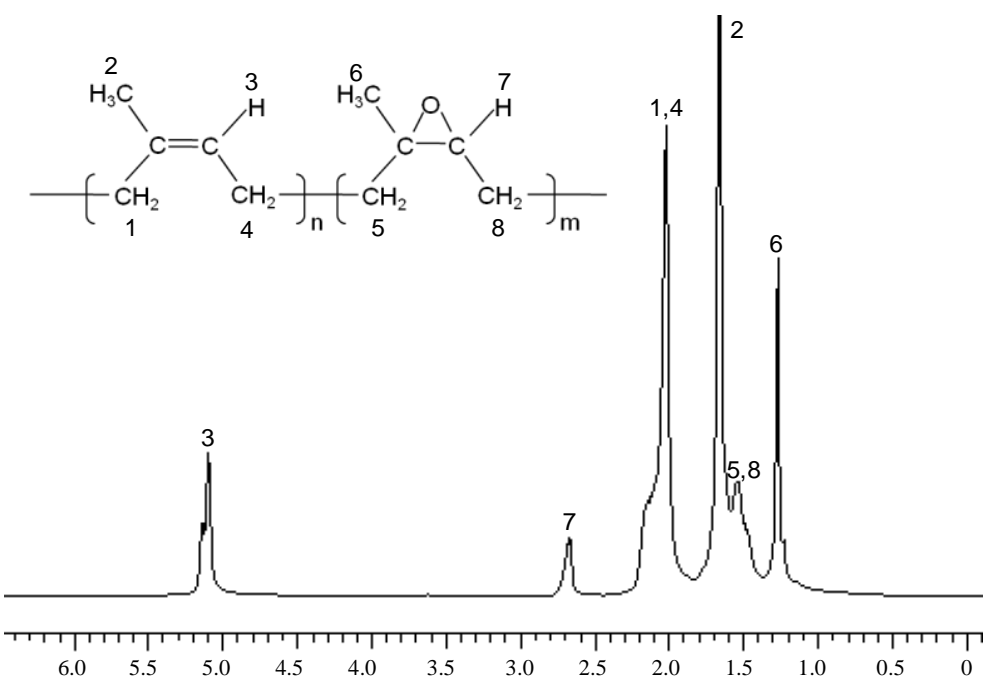

Figure 3.1 Typical ${ }^{1} \mathrm{H}$ NMR spectrum of ENR and its proton type assignment.

The mole\% of epoxide groups was calculated using Equation (3.1):

$$
\text { mol\% of epoxide groups }=\frac{b}{a+b} \times 100
$$


where $a$ is the integrated peak area of the olefinic proton of NR at $5.1 \mathrm{ppm}$ and $\mathrm{b}$ is the integrated peak area of $\mathrm{H}$ attached to the oxirane rings of ENR at $2.7 \mathrm{ppm}$, respectively, as shown in Figure 3.2. The NMR spectra of NR and ENRs with different levels of epoxide groups are shown in Figure 3.2.

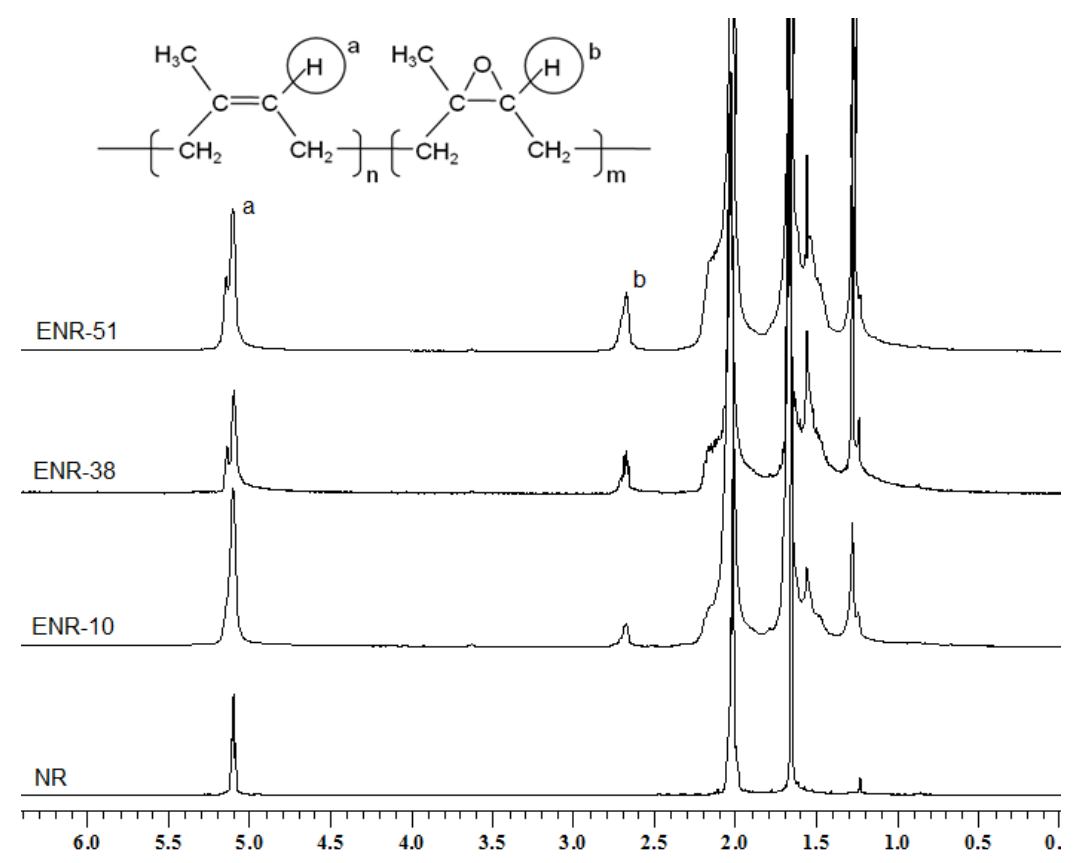

Figure 3.2 ${ }^{1} \mathrm{H}$ NMR spectra of natural rubber (NR) and epoxidized natural rubbers (ENRs) with 10, 38 and 51 mol\% epoxide, denoted as ENR-10, ENR-38 and ENR-51.

\subsubsection{Compound preparation}

Rubber compounds were prepared using the formulations as shown in Table 3.1. The ENR content was varied in a range of 2.5 to $15.0 \mathrm{phr}$. Amounts of TESPT and DPG were calculated relating to the silica CTAB specific surface area, as suggested by Guy et al.. ${ }^{[27]}$ Mixing was carried out using an internal mixer (Brabender Plasticorder 350s) with an initial mixer temperature setting of $110^{\circ} \mathrm{C}$, rotor speed of $60 \mathrm{rpm}$, according to the mixing procedure as shown in Table 3.2. The silica-filled NR compounds with and without TESPT and without ENR were prepared and treated as reference. 
Table 3.1 Compound formulations

\begin{tabular}{llll}
\hline \multicolumn{1}{c}{ Ingredients } & \multicolumn{3}{c}{ Amounts (phr) } \\
\hline RSS 3 & 100.0 & 100.0 & $97.5-85.0$ \\
ENRs & - & - & $2.5-15.0$ \\
Zeosil 1165MP & 55.0 & 55.0 & 55.0 \\
TESPT $^{*}$ & - & 4.5 & - \\
TDAE oil & 8.0 & 8.0 & 8.0 \\
ZnO & 3.0 & 3.0 & 3.0 \\
Stearic acid & 1.0 & 1.0 & 1.0 \\
TMQ & 1.0 & 1.0 & 1.0 \\
DPG* & 1.0 & 1.0 & 1.0 \\
CBS & 1.5 & 1.5 & 1.5 \\
Sulfur & 1.5 & 1.5 & 1.5 \\
\hline
\end{tabular}

${ }^{*}$ Amounts of TESPT and DPG were calculated according to the following equations: ${ }^{[2]}$

TESPT $(p h r)=0.00053 \times Q \times A$ and $D P G(p h r)=0.00012 \times Q \times A$

where $Q$ is the amount of silica (phr) and $A$ is the CTAB surface area of the silica $\left(155 \mathrm{~m}^{2} / \mathrm{g}\right)$.

Table 3.2 Two-step mixing procedure

\begin{tabular}{lc}
\hline Mixing procedure & $\begin{array}{c}\text { Cumulative time } \\
\text { (mins.) }\end{array}$ \\
\hline Step 1 : Internal mixer & 2 \\
- NR and ENR mastication & 7 \\
- Addition of a first half of (silica+TESPT) & 12 \\
- Addition of a second half of (silica+TESPT) and & 15 \\
TDAE oil & \\
- Addition of ZnO, stearic acid, and TMQ & 5 \\
\hline Step 2: Two roll mill & \\
- Addition of DPG, CBS, and sulfur &
\end{tabular}

3.2.4 Mooney viscosity, Payne effect and flocculation rate constant of unvulcanized compounds

Mooney viscosity $\left[\mathrm{ML}(1+4), 100^{\circ} \mathrm{C}\right]$ was tested using a Mooney viscometer (MV 2000VS, Alpha Technologies) according to ASTM D1646. The Payne effect ${ }^{[10]}$ or fillerfiller interaction of the uncured silica-filled compounds were studied by using a Rubber Process Analyzer (RPA 2000, Alpha Technologies) at $100^{\circ} \mathrm{C}$, frequency $0.5 \mathrm{~Hz}$ and varying strains in the range of 0.56 to $100 \%$. The difference of storage moduli at low strain (i.e. $0.56 \%$ ) and high strain (i.e. $100 \%$ ) is reported.

The flocculation rate constant $\left(k_{a}\right)$ of the uncured silica-filled compounds was studied by using the RPA 2000 at $100^{\circ} \mathrm{C}$, strain $0.56 \%$, frequency $1.00 \mathrm{~Hz}$, and test time for 12 minutes. The $k_{a}$ was calculated following Equations (3.2) and (3.3): ${ }^{[28]}$ 


$$
x=\frac{s^{\prime}(t)-s^{\prime}(1)}{s^{\prime}(\infty)-s^{\prime}(1)}
$$

where $x$ is the degree of flocculation, $s^{\prime}(t)$ is the storage modulus at $0.56 \%$ strain at test time t, $s^{\prime}(1)$ is the storage modulus after preheating for 1 minute, and $s^{\prime}(\infty)$ is the storage modulus after heating for 12 minutes.

$$
k_{a}=\frac{\ln \left(1-x_{1}\right)-\ln \left(1-x_{2}\right)}{t_{2}-t_{1}}\left[\min ^{-1}\right]
$$

where $x_{1}$ and $x_{2}$ are the degrees of flocculation at different heating times, i.e. $t_{1}$ and $t_{2}$, respectively.

\subsubsection{Networking factor and interaction parameter}

Silica-filled NR compounds without curatives obtained after the first mixing step were compressed at $150^{\circ} \mathrm{C}$ for 30 minutes to obtain rubber sheets with a thickness of 2 $\mathrm{mm}$, which were then cut into specimens of $40 \times 7 \times 2 \mathrm{~mm}^{3}$ dimensions. The straindependent dynamic mechanical properties were then analyzed by using a dynamic mechanical analyzer (DMA) (Viscoanalyzer, VA2000, Metravib, France) at a constant frequency of $3.5 \mathrm{~Hz}$ at $25^{\circ} \mathrm{C}$ and varying strain amplitudes in the range of $0.083 \%$ to $5.0 \%$. A filler-filler networking factor $(n)$ was calculated from the ratio of storage moduli at $0.083 \%$ and $5.0 \%$ strain of the compounds without curatives.

As proposed by Ayala et al. ${ }^{[29]}$ originally for determination of the carbon blackrubber interaction, a polymer-filler interaction parameter (I) can be calculated from the static and dynamic moduli of a compound according to Equation (3.4):

$$
I=\frac{\sigma}{\eta}
$$

Herein, $\sigma$ is the slope of the stress-strain curve of a cured vulcanizate taken at a relatively linear region at low elongation (i.e. at $10 \%$ elongation in this work). 


\subsubsection{Bound rubber measurement}

$0.25 \mathrm{~g}$ of uncured compound (without curatives) was cut into small pieces, put into a metal cage and immersed in toluene at room temperature for $72 \mathrm{~h}$ (renewed every $24 \mathrm{~h}$ ). The sample was removed from the toluene, dried at $50^{\circ} \mathrm{C}$ for $24 \mathrm{~h}$, then immersed in toluene again for $72 \mathrm{~h}$ at room temperature in either a normal or an ammonia atmosphere. The ammonia treatment was done to cleave the physical linkages between rubber and silica, in order to determine the chemically bound rubber versus bound rubber physical of nature. The sample was finally dried at $50^{\circ} \mathrm{C}$ for $24 \mathrm{~h}$. The bound rubber content was then calculated using the following equation: ${ }^{[30]}$

$$
\text { Bound rubber }(\%)=\frac{\left(m-m_{S}\right)}{m_{r}} \times 100
$$

Where $m$ is the weight of sample after extraction, $m_{s}$ is the weight of silica in the sample and $m_{r}$ is the original weight of rubber in the sample.

\subsubsection{Cure characteristics and tensile properties}

Cure properties of the compounds were studied by using the RPA at $150^{\circ} \mathrm{C}$, frequency $0.833 \mathrm{~Hz}$ and $2.79 \%$ strain for 30 minutes. Then, the compounds were vulcanized to their optimum cure time $\left(t_{c 90}\right)$ by using a Wickert WLP 1600 laboratory compression press at $150^{\circ} \mathrm{C}$ and 100 bars into $2 \mathrm{~mm}$ thick sheets. Type 2 dumb-bell test specimens were die-cut from the press-cured sheets and tensile tests were carried out with a Zwick tensile tester Model Z1.0/TH1S at a crosshead speed of $500 \mathrm{~mm} / \mathrm{min}$ according to ASTM D412.

\subsubsection{Loss tangent at $60^{\circ} \mathrm{C}$ by RPA and dynamic mechanical properties by DMA}

The loss tangent of the vulcanizates was determined using the RPA at $60^{\circ} \mathrm{C}$, strain $3.49 \%$ with varying frequency sweeps in the range of $0.05-33.00 \mathrm{~Hz}$. The samples were cured in the RPA chamber at $150^{\circ} \mathrm{C}$ to reach their optimum cure times before being tested.

Dynamic mechanical properties of silica-filled NR vulcanizates, i.e. storage modulus, loss modulus and tan $\delta$, were characterized with a Metravib Viscoanalyzer VA 2000. The samples were cut into $6 \times 4 \times 2 \mathrm{~mm}^{3}$ specimen and tested in tension mode with a temperature sweep from $-80^{\circ} \mathrm{C}$ to $80^{\circ} \mathrm{C}$ at a frequency of $10 \mathrm{~Hz}$ and $0.1 \%$ strain. 


\subsubsection{FTIR study of silica-filled NR vulcanizates}

The silica-filled NR compounds with ENRs containing different mol\% epoxide as compatibilizer were vulcanized to their optimum cure times before being characterized with ATR-FTIR spectroscopy to investigate the silica-rubber interaction in the compounds. The ENR content in the samples was fixed at $7.5 \mathrm{phr}$, while the mol\% of epoxide groups was varied. The silica-filled NR compounds with TESPT and without compatibilizer were also analyzed for comparison.

\subsubsection{SEM analysis of silica-filled NR vulcanizates}

The dispersion of silica in the NR matrix with ENR as compatibilizers at $7.5 \mathrm{phr}$ was analyzed by Scanning Electron Microscopy (SEM). The silica-filled NR compounds were prepared by reducing silica contents from $55 \mathrm{phr}$ in the normal formation to $30 \mathrm{phr}$ in order to be able to observe the silica dispersion in the NR matrix. The compounds were vulcanized to their optimum cure times, and a newly fractured surface of vulcanizate was created by cryogenic cracking after immersing in liquid nitrogen. The fractured surfaces of the specimens were coated with gold before being analyzed.

\subsection{Results and discussion}

\subsubsection{Mooney viscosity, Payne effect and flocculation tendency}

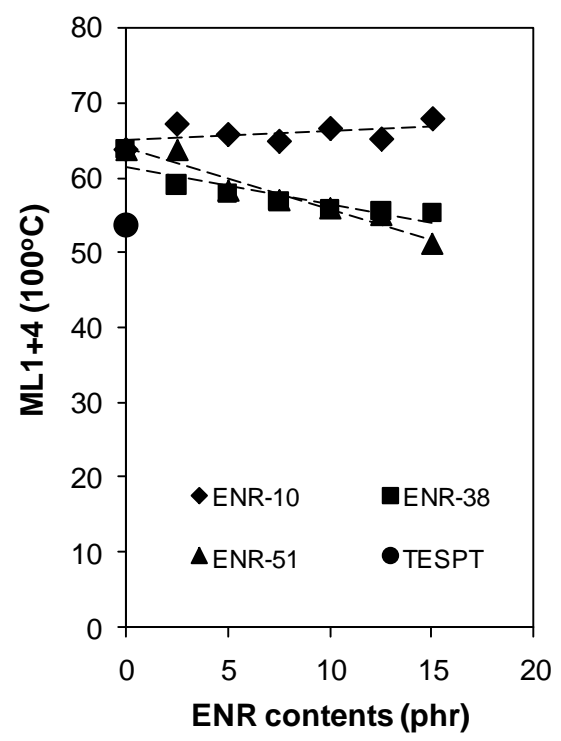

Figure 3.3 Mooney viscosity of silica-filled natural rubber with ENR as compatibilizer. 
The Mooney viscosities of the compounds with ENR-10 as compatibilizer show almost no change when the amount of ENR-10 is increased from 2.5 to 15 phr: Figure 3.3. Incorporation of ENR-38 and ENR-51 decreases the Mooney viscosities of the compounds to match the level of that of the TESPT-containing compound when the amount of ENRs used is $\geq 10$ phr. The decrease of compound viscosity when ENR is used as compatibilizer implies an improvement of silica dispersion due to interaction between epoxide groups and silanol groups of the silica resulting in better compatibility. However, the compound viscosities can also be affected by various factors including the NR/ENR blend incompatibility. The presence of polar epoxy groups in the ENRs leads to a difference between the solubility parameters of NR and ENR, and increases interfacial tension. It has been reported that the NR/ENR blends exhibit two distinct glass transition temperatures $\left(\mathrm{T}_{\mathrm{g}}\right)$ corresponding to the original polymers, as analyzed by differential scanning calorimetry (DSC) ${ }^{[31]}$, and dynamic mechanical analysis (DMA). ${ }^{[32]}$
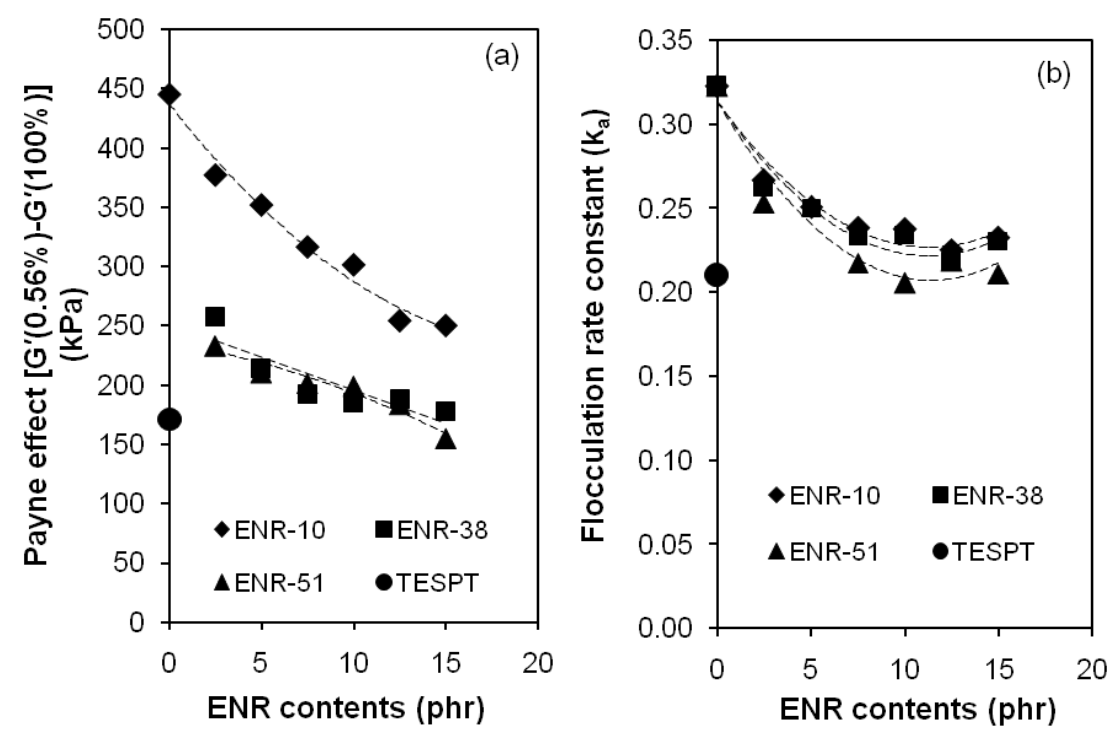

Figure 3.4 Payne effect (a) and flocculation rate constant $\left(\mathrm{k}_{\mathrm{a}}\right)(\mathrm{b})$ of silica-filled natural rubber with ENR as compatibilizer.

Filler-filler interaction, as indicated by the Payne effect in Figure 3.4(a), of the filled compounds also decreases when the ENR content is increased. The compound without any compatibilizer shows the highest filler-filler interaction. ENR-10 as compatibilizer already shows a strong reduction of the Payne effect with increasing content. The compounds with ENR-38 and ENR-51 show similar values of the Payne 
effect which is much lower compared to the compounds containing ENR-10. The lower Payne effect indicates less silica-silica interactions, usually indicating better dispersion of silica in the matrix. The incorporation of ENR-38 and ENR-51 in the range of 7.5 to $15 \mathrm{phr}$ results in compounds that have similar Payne effects compared to the compound with TESPT. The reduction of filler-filler interaction in the presence of ENR in silica-filled NR compounds can again be attributed to interactions between the silanol groups of silica and epoxide groups of ENR through hydrogen bonding and/or chemical bonding that occur after possible ring opening of the epoxy ring either during mixing or compression molding, as previously proposed by Manna et al. ${ }^{[33]}$ and Rocha et al.. ${ }^{[12]} \mathrm{A}$ possible mechanism for bonding between the ENR and silica is shown in Scheme 3.1, and the model of interactions and/or reactions between the silanol groups of silica and ENR molecules in the NR matrix is proposed in Scheme 3.2.

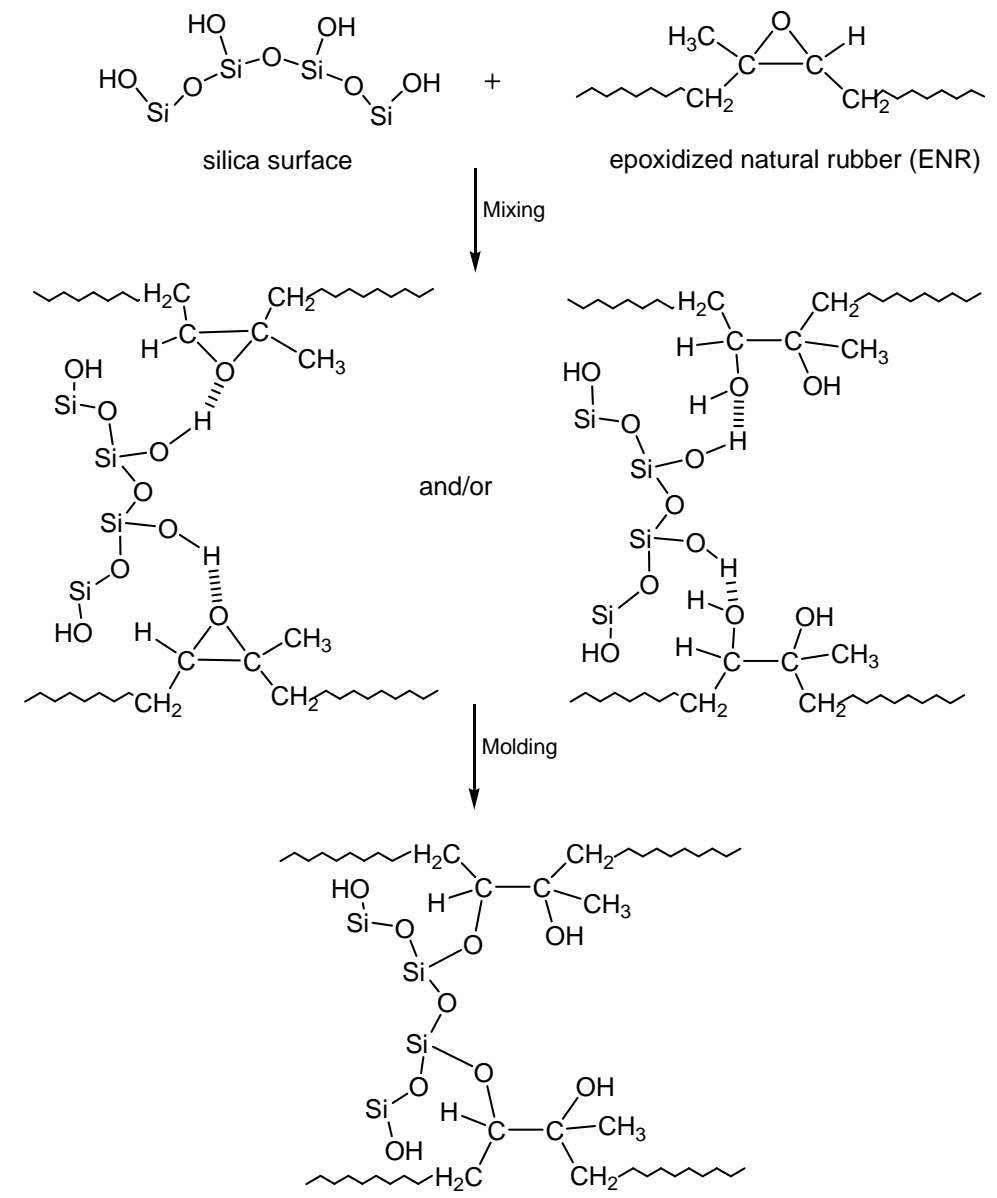

Scheme 3.1 Possible mechanism for interactions and bonding between ENR and silica. 


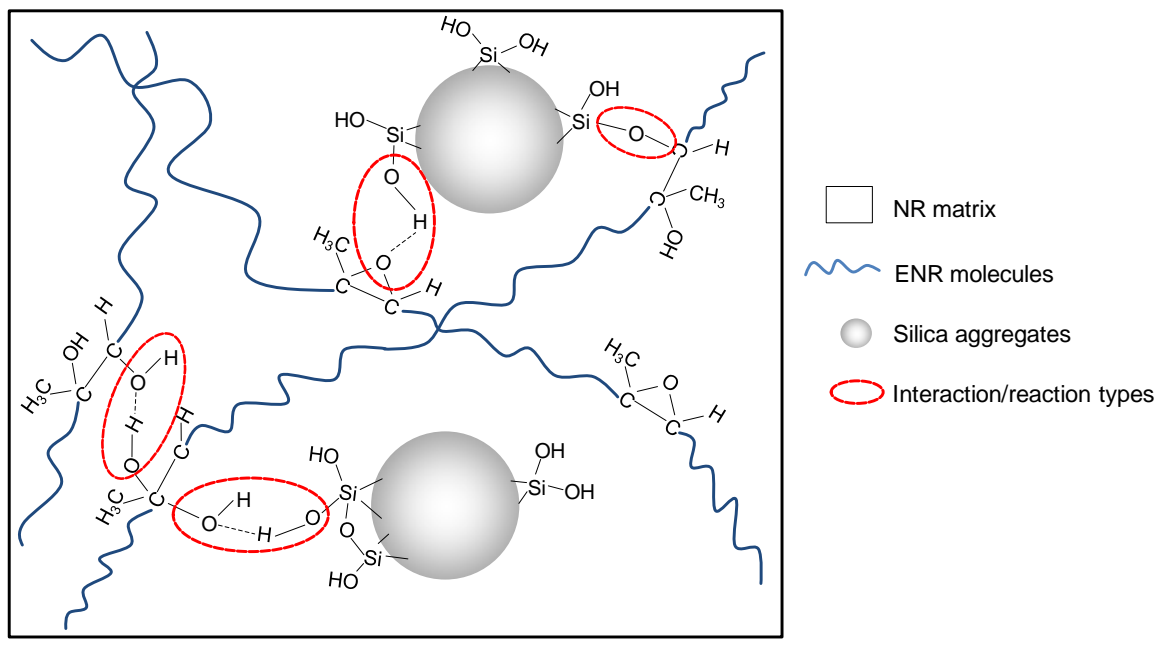

Scheme 3.2 Proposed model of interactions and bonding between silanol groups on the silica surface and ENR molecules in NR matrix.

Due to the presence of silanol groups on the silica surface that can form hydrogen bonds between silica aggregates, these aggregates can again form agglomerates after mixing. This reformation of filler network in the compound is also called filler flocculation. The flocculation process of the silica-silica network strongly increases with heat treatment. ${ }^{[28,34]}$ The results in Figure 3.4(b) show that the silica-filled compound with neither ENR nor TESPT has the highest flocculation rate constant $\left(\mathrm{k}_{\mathrm{a}}\right)$ compared to the other compounds, because of the strong hydrogen bonding between the silica aggregates. ${ }^{[35]}$ After the ENRs are added into the silica-filled compounds, the flocculation rate constant decreases, especially when ENR-51 is increased to $7.5 \mathrm{phr}$, which compound shows almost the same level of $k_{a}$ compared to the silica-filled compound with TESPT. The results confirm that the use of ENR as compatibilizers can effectively reduce filler-filler interaction in the compounds leading to a reduction of filler flocculation of the compounds. 


\subsubsection{Networking factor and interaction parameter}

Table 3.3 Storage modulus $\left(E^{\prime}\right)$ at low strain $(0.083 \%)$ and high strain $(5.0 \%)$ and $\Delta E^{\prime}$ at $25^{\circ} \mathrm{C}$

\begin{tabular}{lccc}
\hline \multicolumn{1}{c}{ Sample } & $\begin{array}{r}\mathbf{E}^{\prime} \text { at } \mathbf{0 . 0 8 3 \%} \text { strain } \\
(\mathbf{M P a})\end{array}$ & $\begin{array}{c}\mathbf{E}^{\prime} \text { at 5.0\% strain } \\
(\mathbf{M P a})\end{array}$ & $\begin{array}{c}\Delta \mathbf{E}^{\prime} \\
(\mathbf{M P a})\end{array}$ \\
\hline Without compatibilizer & 42.40 & 7.96 & 34.44 \\
ENR-10 7.5 phr & 27.00 & 5.24 & 21.76 \\
ENR-38 7.5 phr & 26.70 & 6.73 & 19.97 \\
ENR-51 7.5 phr & 20.60 & 5.75 & 14.85 \\
TESPT 4.5 phr & 8.37 & 2.89 & 5.48 \\
\hline
\end{tabular}
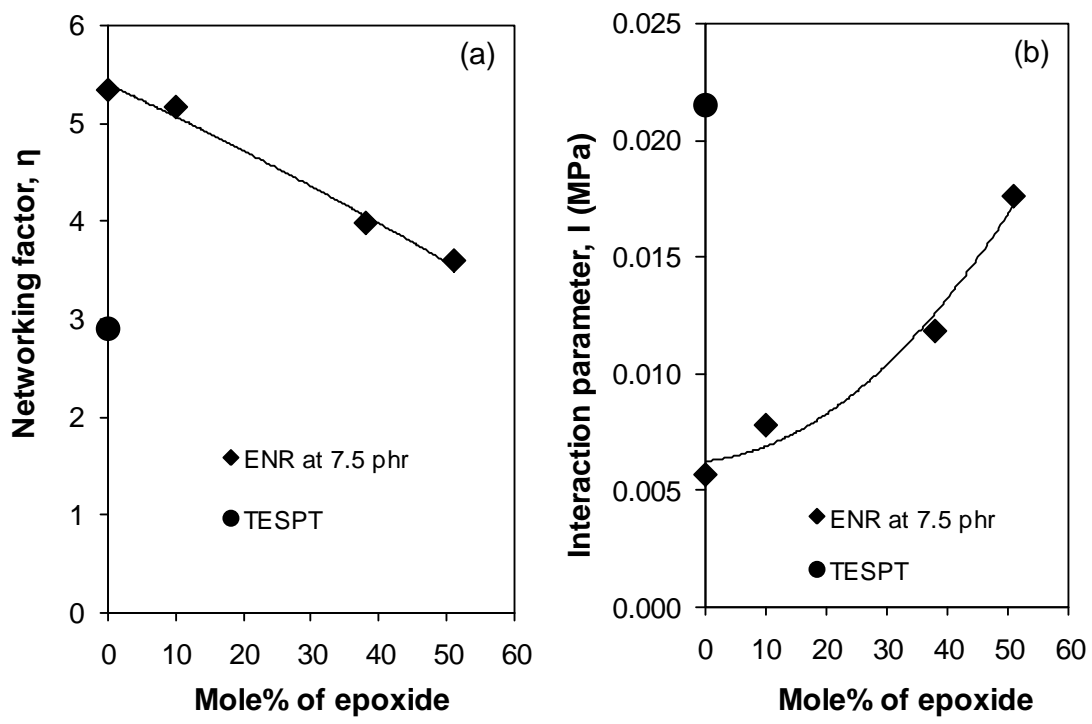

Figure 3.5 Filler networking factor (a) and rubber-filler interaction parameter (b) of silicafilled natural rubber with ENR at $7.5 \mathrm{phr}$ as compatibilizer in comparison with the vulcanizates containing TESPT silane coupling agent.

The variation of storage modulus with \% strain of silica-filled vulcanizates has been related to the networking factor and interaction parameter of the filled samples. ${ }^{[29,36]}$ The decrease of storage modulus with increasing strain amplitude infers that the filler-filler network in the rubber matrix is broken down. The storage modulus at low strain is strongly affected by the filler-filler interaction in the system, while at high strain amplitude after the break down of filler-filler network such property is only affected by filler-rubber 
interaction, the filler volume fraction and rubber network in the sample. ${ }^{[10,37]}$ As shown in Table 3.3, the difference of storage moduli at $5.0 \%$ and $0.083 \%$ strain $\left(\Delta \mathrm{E}^{\prime}\right)$ of the silicafilled NR vulcanizates decreases with increase of mole\% of epoxide groups, compared to the silica-filled NR without any compatibilizer. Herein, the rubber with TESPT silane coupling agent shows the lowest $\Delta \mathrm{E}^{\prime}$. Figure 3.5(a) shows the networking factor $(\eta)$ of silica-filled NR compounds with ENRs as compatibilizer. The results show a slight decrease of filler-filler networking factor ( $\eta$ ) when ENR-10 was added into the compounds, but a strong decrease of $\eta$ when 7.5 phr of ENR-38 and ENR-51 was used. This again indicates that the epoxide groups of ENRs interact and/or react with silanol groups on the silica surface leading to a reduction of silica-silica interaction or silica agglomeration in the NR matrix and an improvement of silica dispersion. When considering the rubber-filler interaction parameter (I) which is defined by the ratio of static to dynamic moduli of the filled-rubber ${ }^{[29]}$, as shown in Figure 3.5(b), the values of I increase with mole\% of epoxide groups of ENRs, but the ultimate effect is lower than that of the silica-filled compound with TESPT. In the silica-silane system, TESPT can form covalent bonds with the silica surface via the silanization reaction between ethoxy groups of TESPT and $\mathrm{Si}-\mathrm{OH}$ groups of silica, and generate crosslinks with the rubber phase via sulfur atoms in its structure. ${ }^{[38-39]}$ Even though the use of TESPT provides the lowest filler-filler interaction as indicated by the lowest Payne effect: Figure 3.4(a), the flocculation rate constant: Figure 3.4(b), the filler networking factor: Figure 3.5(a) and highest filler-rubber interaction parameter: Figure 3.5(b), the use of ENRs as compatibilizer clearly demonstrates that the epoxy groups on the main chain of ENR can effectively reduce the filler-filler interactions. By increasing the epoxide groups in the system, the polymer-filler interaction parameter (I) increases whilst the Payne effect, flocculation rate constant and filler-filler networking factor $(\eta)$ decrease. 


\subsubsection{Filler-rubber interaction}

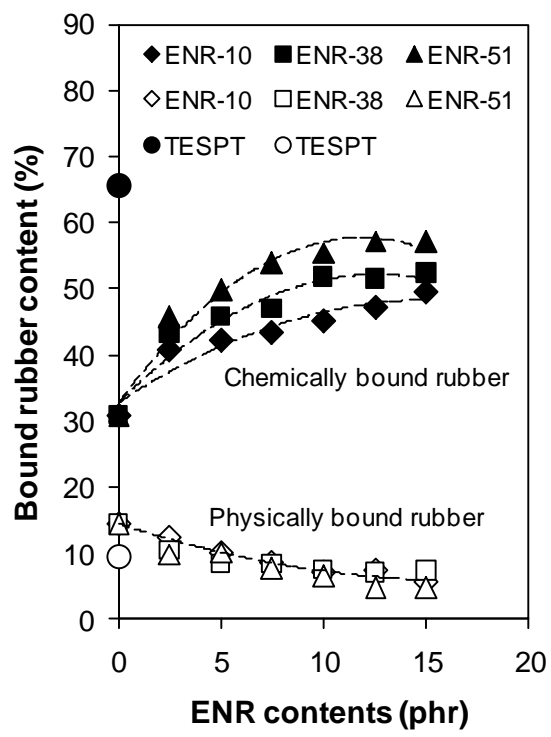

Figure 3.6 Physically and chemically bound rubber contents of silica-filled NR with ENR as compatibilizer.

The chemically bound rubber contents of the silica-filled NR significantly increase while the physically bound rubber contents slightly decrease by increasing the ENR contents and mole\% of epoxide on ENR, as shown in Figure 3.6. When only NR is used, the addition of TESPT coupling agent leads to a remarkably high chemically bound rubber content, compared to the ones without. The addition of ENR into the NR compounds without TESPT clearly improves the bound rubber content, i.e. filler-rubber interaction. These results prove that ENR can generate chemical interaction and/or reaction in silicafilled NR, according to the mechanism as proposed in Scheme 3.1. The use of ENR-51 in the range of 7.5-15 phr results in an optimum chemically bound rubber content. This is in accordance with the Payne effect: Figure 3.4(a) and flocculation rate constant results: Figure 3.4(b), attributed to the compatibilization effect of ENR. For the NR compound without ENR or TESPT, there is still chemically bound rubber seen in Figure 3.6. This can be mainly attributed to the nature of NR itself which has the terminal groups associated with proteins and phospholipids ${ }^{[40]}$ that can possibly interact with the silanol groups of the silica surface via hydrogen bonding. The interactions between silica and proteins present in NR has been clearly demonstrated recently. ${ }^{[41]}$ 


\subsubsection{Cure properties}
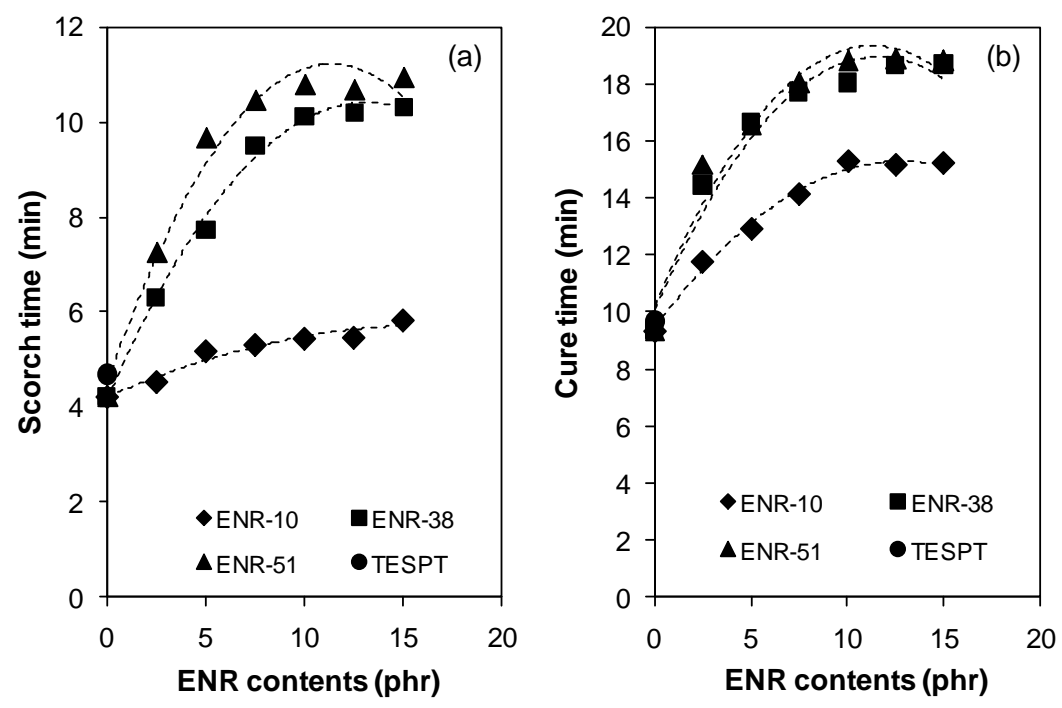

Figure 3.7 Scorch (a) and optimum cure times (b) of silica-filled NR with ENR as compatibilizer.

Scorch time $\left(t_{s 1}\right)$ and optimum cure time $\left(t_{c 90}\right)$ of the compounds are shown in Figures $3.7(\mathrm{a})$ and $3.7(\mathrm{~b})$, respectively. Both scorch and optimum cure times of the compounds are prolonged when ENR contents and mole\% of epoxide are increased. This might be due to the high polarities of ENR and silica which interfere with the vulcanization. Even though part of the silanol groups are assumed to have interacted with epoxide groups, the remaining free silanol groups as well as free epoxide groups can possibly form hydrogen bonds with polar accelerators, causing accelerator adsorption on the polar surface. In addition, due to the polarity difference between NR and ENR, the more polar curatives can migrate into the ENR phase, and so cause a delay of the vulcanization reaction in the NR matrix. The silica filled-compound with TESPT displays shorter scorch and cure times, because TESPT itself can act as sulfur donor and optimized mixing conditions were employed to ensure a good silanization reaction. 


\subsubsection{Tensile properties}

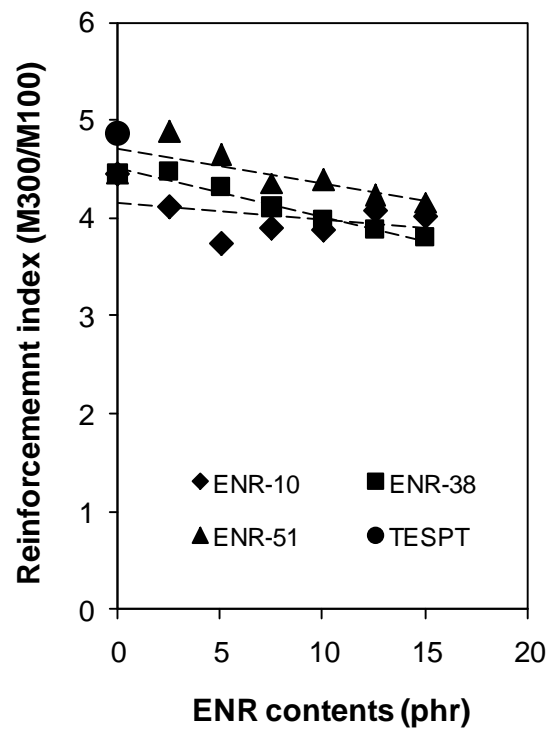

Figure 3.8 Reinforcement index of silica-filled natural rubber with ENR as compatibilizer.

The silica-filled NR with TESPT shows a higher reinforcement index (M300/M100) when compared to the compounds with ENRs as compatibilizers: Figure 3.8, indicating a greater extent of interactions between the silica and rubber phases when the silane coupling agent is used, as also previously observed by the chemically bound rubber content: Figure 3.6. When compared among the ENR types, the use of ENR-51 gives the highest reinforcement index. An increase of ENR content tends to decrease the reinforcement index slightly. This may be caused by the presence of two different rubber phases in the system due to the incompatibility between NR and ENR ${ }^{[31,32]}$, as previously mentioned in Section 3.1. 

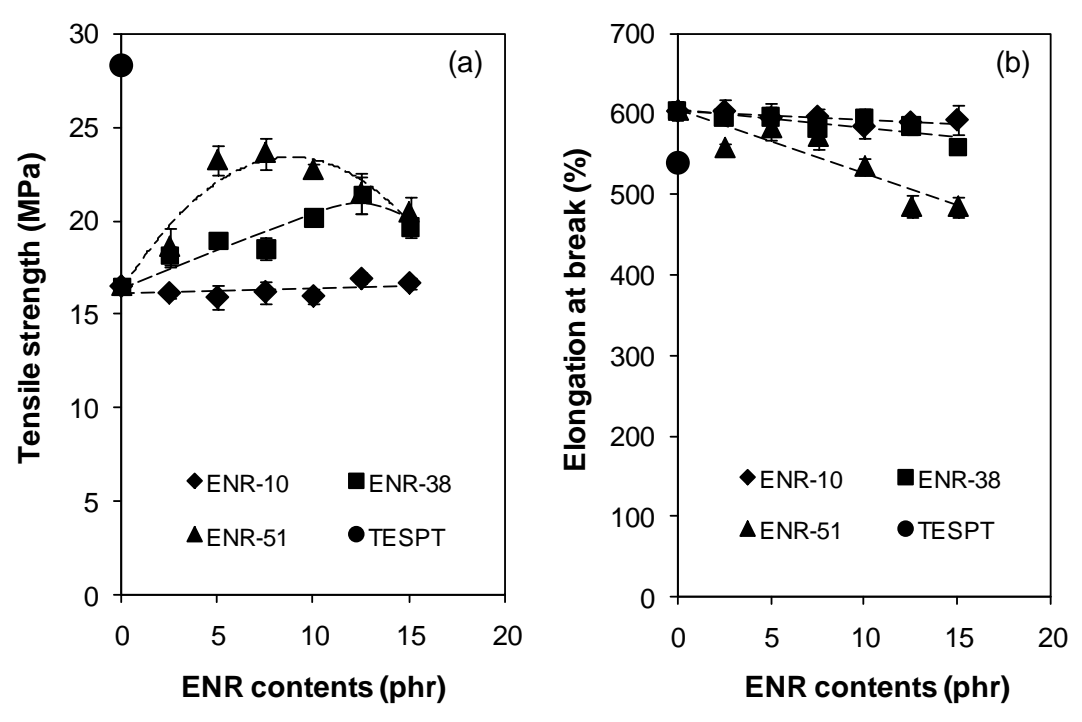

Figure 3.9 Tensile strength (a) and elongation at break (b) of silica-filled natural rubber with ENR as compatibilizer.

The use of ENR-10 shows no positive effect on the tensile strength of the silicafilled NR, but the addition of ENR-38 and ENR-51 enhances the tensile strength, compared to the compound without compatibilizer: Figure 3.9(a). ENR-51 gives a higher tensile strength than ENR-38, and increasing the ENR content increases tensile strength to a maximum whereafter the property drops. The optimum value is observed at $7.5 \mathrm{phr}$ for ENR-51 and 12.5 phr for ENR-38. The results support the proposition that the epoxide functional groups improve silica-rubber interaction in the compounds. This is in accordance with the increased bound rubber content in Figure 3.6 and the decreased elongation at break as shown in Figure 3.9(b). 


\subsubsection{Dynamic mechanical properties}

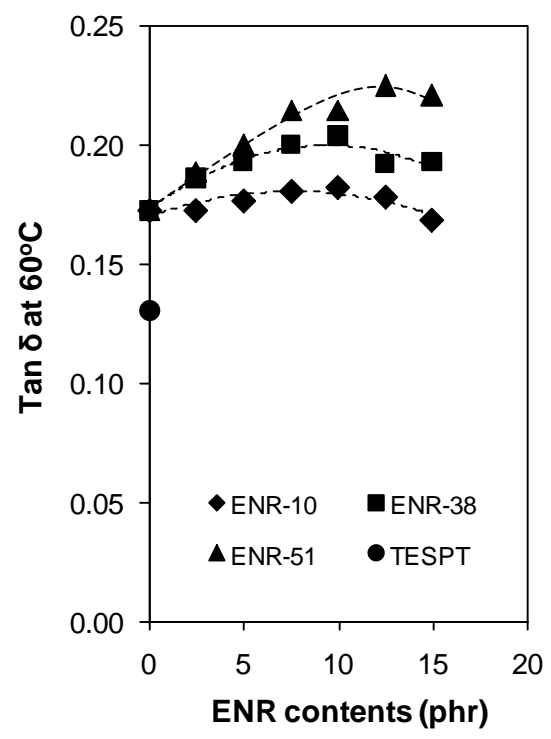

Figure 3.10 Tan $\delta$ at $60^{\circ} \mathrm{C}$ of silica-filled NR with ENR as compatibilizer.

The loss tangent or tan $\delta$ at $60^{\circ} \mathrm{C}$ of the vulcanizates is an indication of tire rolling resistance in a laboratory scale test. The compound with lower tan $\delta$ at $60^{\circ} \mathrm{C}$ implies a lower rolling resistance of tires made thereof. The tan $\delta$ of silica-filled NR vulcanizates at $10 \mathrm{~Hz}$, as shown in Figure 3.10, reveal that the compound with TESPT shows an outstanding low rolling resistance compared to the other compounds because of good silica dispersion and silica-to-rubber bonding via the silane molecule. On the other hand, the silica-filled NR compounds with ENRs as compatibilizers show an increase of tan $\delta$ with mole\% of epoxide content. The addition of ENR-10 results in a similar $\tan \delta$, compared to the compound without coupling agent, and shows no strong dependence on ENR-10 loading. But the use of ENR-38 and ENR-51 as compatibilizer clearly increases $\tan \delta$ which negatively affects the tire rolling resistance. This increase of $\tan \delta$ is associated with the damping properties of ENRs as the glass transition temperature of this material is shifted to higher values with increasing mol\% epoxide. ${ }^{[13,26]}$ The glass transition temperatures $\left(\mathrm{T}_{\mathrm{g}}\right)$ of ENR-10, ENR-38 and ENR-51 are approximately $-60{ }^{\circ} \mathrm{C}$, $30{ }^{\circ} \mathrm{C}$ and $-25^{\circ} \mathrm{C}$, respectively. ${ }^{[26]}$ However, the $\mathrm{T}_{\mathrm{g}}$ of reinforcing ENR-50 with silica occurs at $-3^{\circ} \mathrm{C}$ and either the increase of molding time or addition of silane coupling agent influences on shifting the $T_{g}$ of silica-filled ENR-50 to higher temperature. ${ }^{[36]}$ It is due to the movement of rubber segments is restricted by the present of chemically bonding of rubber 
chains to silica surfaces. ${ }^{[36,42]}$ In addition, the incorporation of a second polymer phase with different polarity compared to the matrix normally leads to a phase separation and has an influence on dynamic properties. ${ }^{[43]}$ The weaker interfacial adhesion leads to more energy losses under dynamic deformation and so a higher loss tangent.

The dynamic mechanical properties as a function of temperature of silica-filled NR with 7.5 phr of various ENRs as compatibilizers are shown in Figures 3.11-3.12. The temperature dependence of dynamic storage modulus ( $\left.E^{\prime}\right)$ and loss modulus ( $\left.E "\right)$ of silicafilled NR vulcanizates is shown in Figure 3.11. The silica-filled NR vulcanizate without compatibilizer shows the lowest storage modulus due to the poorest interfacial adhesion between silica and rubber. Its strongest filler-filler interactions as indicated by Payne effect (Figure 3.4(a)) suggest a formation of silica agglomerates which introduce voids and pores that result in a decrease of storage modulus of the materials. ${ }^{[44]}$ The storage moduli of the vulcanizates with TESPT and ENRs in the rubbery region are clearly higher than that of the rubber without compatibilizer. The vulcanizates with ENR-38 and ENR-51 as compatibilizer show a two-step change of $E^{\prime}$ and $E^{\prime \prime}$ due to a second glass transition temperature introduced by the ENRs. The interactions between silica and rubber phases as well as between ENR molecules result in the higher $E^{\prime}$, but due to the damping behavior of the ENRs ${ }^{[13,26]}$, the NR vulcanizates with ENRs as compatibilizer show higher E" when compared to the one with TESPT. Moreover, the immiscibility between NR and ENR, ${ }^{[45]}$ contributes to a higher loss of energy and so a loss modulus $E^{\prime \prime}$ as shown in Figure 3.11(b). 

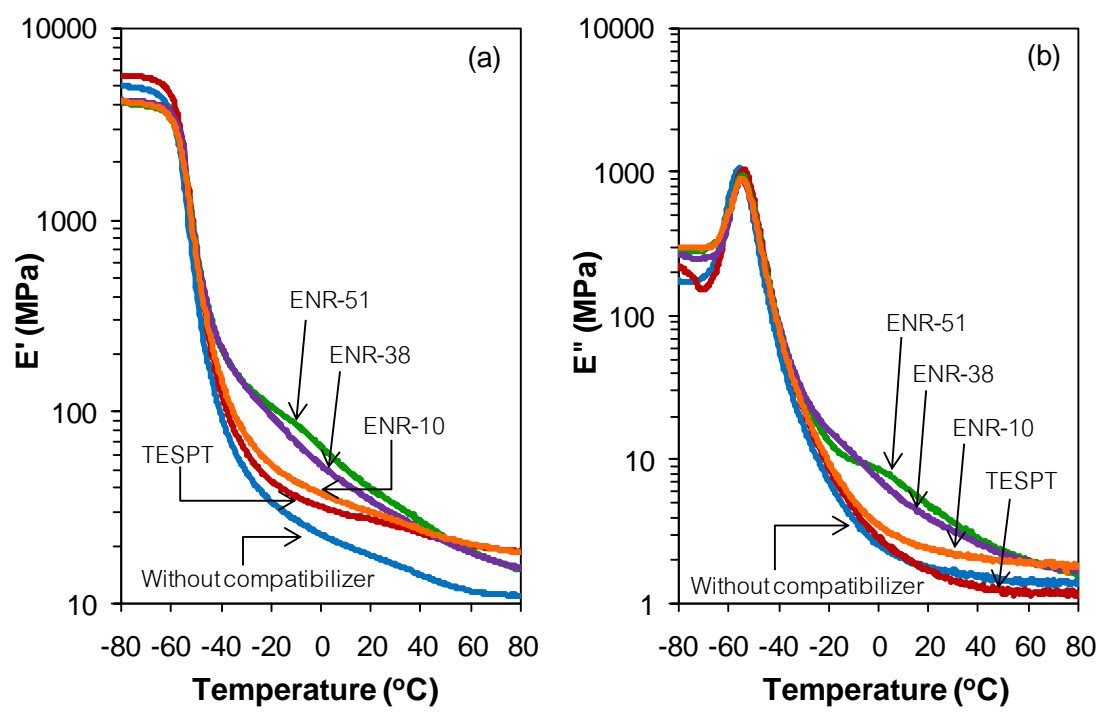

Figure 3.11 Storage modulus (a) and loss modulus (b) of silica-filled NR vulcanizates with 7.5 phr of ENRs as compatibilizer.

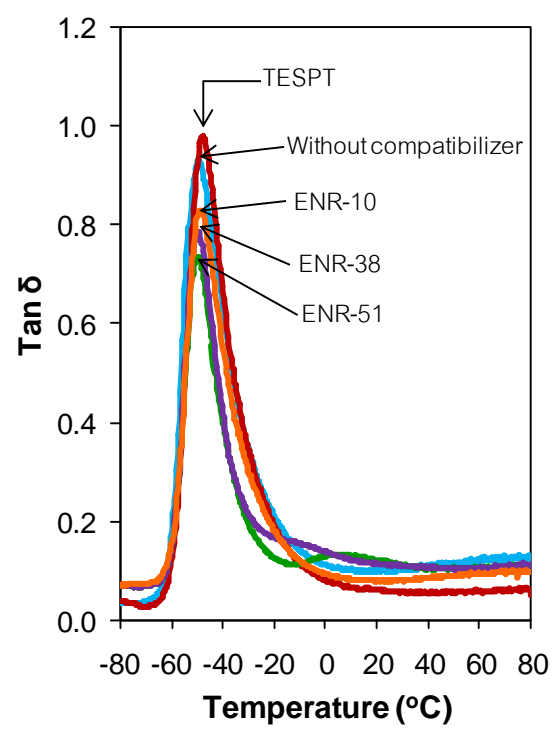

Figure 3.12 Tan $\delta$ as a function of temperature of silica-filled NR vulcanizates with $7.5 \mathrm{phr}$ of ENRs as compatibilizer. 
Figure 3.12 shows the effect of ENR compatibilizers in silica-filled NR vulcanizates on the loss tangent $(\tan \delta)$ as a function of temperature. The addition of 7.5 phr of ENRs with different levels of mole\% epoxide results in a different response of the $\tan \delta$ curves. The height of the tan $\delta$ peaks is decreased when the epoxide group level is increased. This is caused by the interactions between silica and ENRs in the NR matrix and between ENR molecules themselves, leading to a restriction of the molecular chain mobility and relaxation. Furthermore, the higher epoxide group content leads to a possible self-crosslinking between the ENR itself and introduces an immobilization of the rubber chains. Second transitions of tan $\delta$ are observed when ENR-38 and ENR-51 were added into the silica-filled NR compounds, in accordance with the second step changes of $E^{\prime}$ and $E "$ as observed in Figure 3.11. These are again attributed to the glass transition temperatures of ENR-38 and ENR-51. The highest tan $\delta$ peak is observed for the silica/TESPT-filled vulcanizate, which can be described by its better silica dispersion causing a decrease of rubber trapped within the filler network and more mobility of rubber segments in the matrix that involve in the relaxation process. The change of tan $\delta$ peak height with respect to compatibilizer types, i.e. TESPT and epoxidized rubbers, are in accordance with the work previously reported by Saramolee et al. ${ }^{[46]}$

Table 3.4 Transition temperatures at loss modulus and tan $\delta$ peaks, and values of tan $\delta$ at different temperatures of silica-filled NR vulcanizates with various compatibilizers.

\begin{tabular}{lccccc}
\hline \multirow{2}{*}{ Compatibilizer types } & \multicolumn{2}{c}{$\begin{array}{c}\text { Glass transition } \\
\text { temperature }\left({ }^{\circ} \mathbf{C}\right)\end{array}$} & \multicolumn{3}{c}{ Values of tan $\delta$} \\
\cline { 2 - 7 } & at E" peak & at tan $\mathbf{\delta}$ peak & at $\mathbf{0}^{\circ} \mathbf{C}$ & at $\mathbf{5}^{\circ} \mathbf{C}$ & at $\mathbf{6 0 ^ { \circ } \mathbf { C }}$ \\
\hline Without compatibilizer & -56 & -50 & 0.11 & 0.11 & 0.12 \\
ENR-10 7.5 phr & -55 & -50 & 0.09 & 0.09 & 0.09 \\
ENR-38 7.5 phr & -55 & -50 & 0.14 & 0.13 & 0.10 \\
ENR-51 7.5 phr & -54 & -50 & 0.13 & 0.13 & 0.11 \\
TESPT & -53 & -48 & 0.08 & 0.07 & 0.06 \\
\hline
\end{tabular}

The temperatures at peak of loss modulus (E") and tan $\delta$ can be taken as glass transition temperatures $\left(\mathrm{T}_{\mathrm{g}}\right)$ of the materials. As reviewed by Robertson and Roland ${ }^{[47]}, \mathrm{T}_{\mathrm{g}}$ based on the $\tan \delta$ peak can be problematic because tan $\delta$ in the glass-to-rubber softening region is influenced not only by the local segmental polymer dynamics but also by filler induced changes in both storage and loss moduli at higher temperatures. Due to some discrepancies in $T_{g}$ position, this work reports the $T_{g}$ values of silica-filled NR vulcanizates with and without compatibilizers taken from both $E^{\prime \prime}$ and tan $\delta$ peaks, as shown in Table 3.4. It can be seen that the $T_{g}$ 's taken from the $E$ " peaks show a small but 
gradual change of values with compatibilizer types. The silica-filled NR without compatibilizer shows the lowest $T_{g}$ and the presence of compatibilizers tends to shift the $\mathrm{T}_{\mathrm{g}}$ slightly to higher temperature in accordance with the degree of interactions as observed by bound rubber content in Figure 3.6. The silica/TESPT-filled NR vulcanizate has the highest $T_{g}$, while the ENR-compatibilized vulcanizates show similar $T_{g}$ values. The shift of $T_{g}$ to higher temperature is due to the strong interactions between filler and rubber phases leading to restricted movement of the rubber molecules at the interface. ${ }^{[48]}$

Tan $\delta$ in the low temperature range e.g. in the range of $0-20^{\circ} \mathrm{C}$ can be used to indicate wet grip or resistance between tire tread and road surface. A higher tan $\delta$ at low temperature means a better wet grip of a tire. The use of ENR-38 and ENR-51 as compatibilizers in silica-filled NR shows an increase of $\tan \delta$ values at low temperature (Table 3.4) due to the effect of the glass transition temperatures and damping properties of ENR-38 and ENR-51 in the NR matrix. The incorporation of ENR-38 and ENR-51 positively affects tire wet grip but negatively influences tire rolling resistance, as observed by the higher tan $\delta$ values at $60^{\circ} \mathrm{C}$ when compared to the silica-filled NR vulcanizate with TESPT. The results at $60^{\circ} \mathrm{C}$ are in good agreement with the tan $\delta$ values tested by RPA (Figure 3.10), as previously discussed.

\subsubsection{FTIR spectra of silica-filled NR vulcanizates}

As reflected in the changes of properties of both compounds and vulcanizates: Figures 3.3-3.10, the epoxide groups of ENR interact with the silanol groups of silica and promote compatibilization. In order to gain more evidence of such interactions between the epoxide groups of ENR and the silanol groups of silica, vulcanized sheets of silicafilled NR containing either no compatibilizer, TESPT or 7.5 phr of ENR-10, ENR-38 and ENR-51 were subjected to ATR-FTIR analysis to look for a change of absorption peak intensities of the functional groups of silica. The spectra of the vulcanizates in the region of absorption bands that are associated with -OH and Si-O-Si stretching vibrations are shown in Figure 3.13. Herein, Figure 3.13(a) shows the -OH stretching region at 3500$3000 \mathrm{~cm}^{-1}$ and Figure 3.13(b) displays the absorption band of Si-O-Si asymmetric stretch in the range of $1300-900 \mathrm{~cm}^{-1}$. $[8,49]$ 

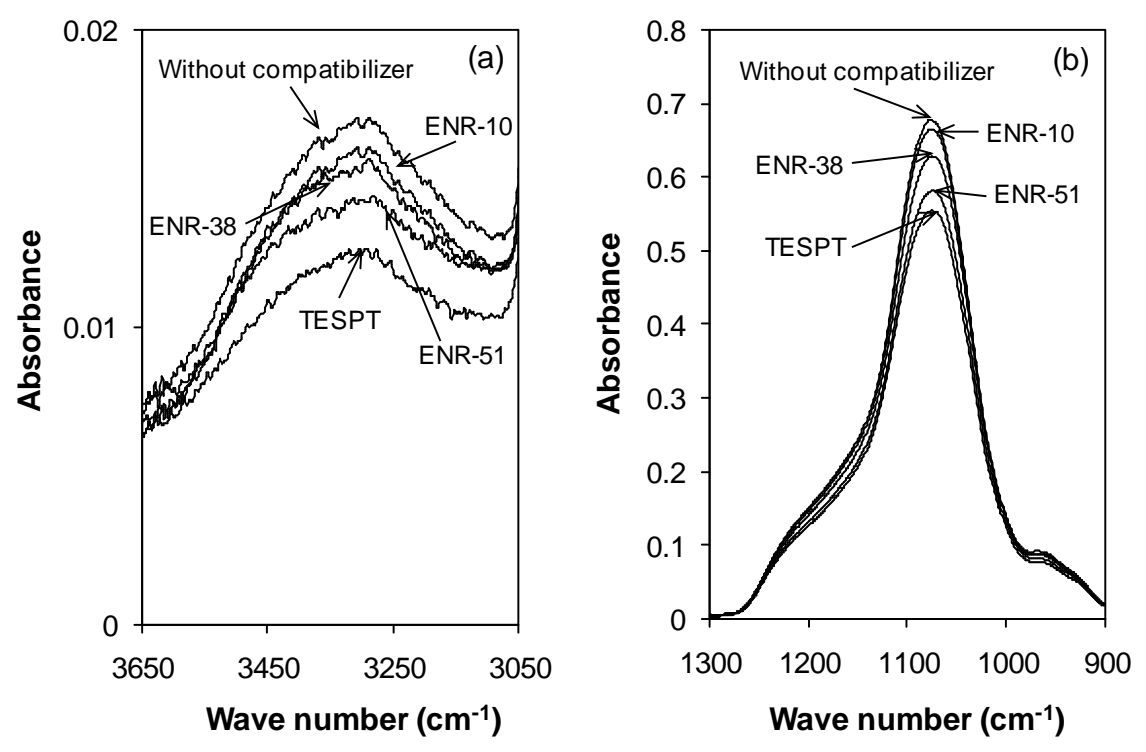

Figure 3.13 ATR-FTIR spectra of silica-filled NR vulcanizates with $7.5 \mathrm{phr}$ of ENR as a compatibilizer compared to that of the vulcanizates with TESPT and without any compatibilizer.

The ATR-FTIR spectra in Figure 3.13(a) show a reduction of -OH groups of silica with increase of mol\% epoxide and use of TESPT. In addition to hydrogen bonding between silanol groups and oxirane oxygen of ENR, as proposed in Scheme 3.1 and Scheme 3.2, $-\mathrm{OH}$ groups of silica can react with $-\mathrm{OH}$ groups of ENR after ring opening, and form bonding during molding. Therefore, the free $-\mathrm{OH}$ of silanol groups decrease. According to Bertora et al. ${ }^{[50]}$ and Zhuravlev et al. ${ }^{[51]}$, the surface silanol groups content of Zeosil $1165 \mathrm{MP}$ is $4.90 \mathrm{OH} \cdot \mathrm{nm}^{-2}$. As only part of these silanol groups are able to react or interact with epoxide groups of ENR for reason of steric hindrance, providing the same content of ENR compatibilizers with varying mol\% epoxide leads to more epoxide groups available to interact, and less free silanol groups remaining in the matrix. As a result, a lower intensity of the $-\mathrm{OH}$ peak with increasing mol\% of epoxide is observed in Figure $3.13(\mathrm{a})$.

The peak intensity of Si-O-Si asymmetric stretching of silica in the NR matrix at $1080 \mathrm{~cm}^{-1}$ decreases with the addition of ENR as compatiblizer and TESPT as coupling agent. The higher the mol\% epoxide of ENR, the more the decrease of peak intensity, while TESPT shows the lowest peak intensity. In Figure 3.13(b), the intensities of the peak shoulder at $965 \mathrm{~cm}^{-1}$ arising from the $\mathrm{Si}-\mathrm{O}$ of the $\mathrm{Si}-\mathrm{OH}$ group also change in the same 
order as observed for the peak at $1080 \mathrm{~cm}^{-1}$ when ENR and TESPT are added. This is also due to the reduction of the amount of $\mathrm{Si}-\mathrm{O}-\mathrm{Si}$ and $\mathrm{Si}-\mathrm{O}$ bonds in the compounds. Thus the use of ENR and TESPT in the silica-filled NR compounds reduces silica-silica interaction leading to a lower number of silica agglomerates in the compounds.

\subsubsection{SEM images of silica-filled NR with ENR as compatibilizers}

Filler dispersion and distribution is an important factor that influences the properties of filled-compounds and vulcanizates. Silica primary particles themselves have a dimension in the nanometer range, but due to the highly polar silanol groups on its surface, silica can easily form aggregates and agglomerates. ${ }^{[48]}$ The hydrophilic character of silica makes it difficult to uniformly disperse and distribute within hydrocarbon rubbers in the absence of any surface modifiers or compatibilizers. The silica dispersion in the NR matrix with a variation of ENR types as compatibilizer is shown in Figure 3.14. In this study, the silica content in the compounds was reduced from $55 \mathrm{phr}$ in the normal formulation to $30 \mathrm{phr}$ for a better clarification of the silica in the matrix.
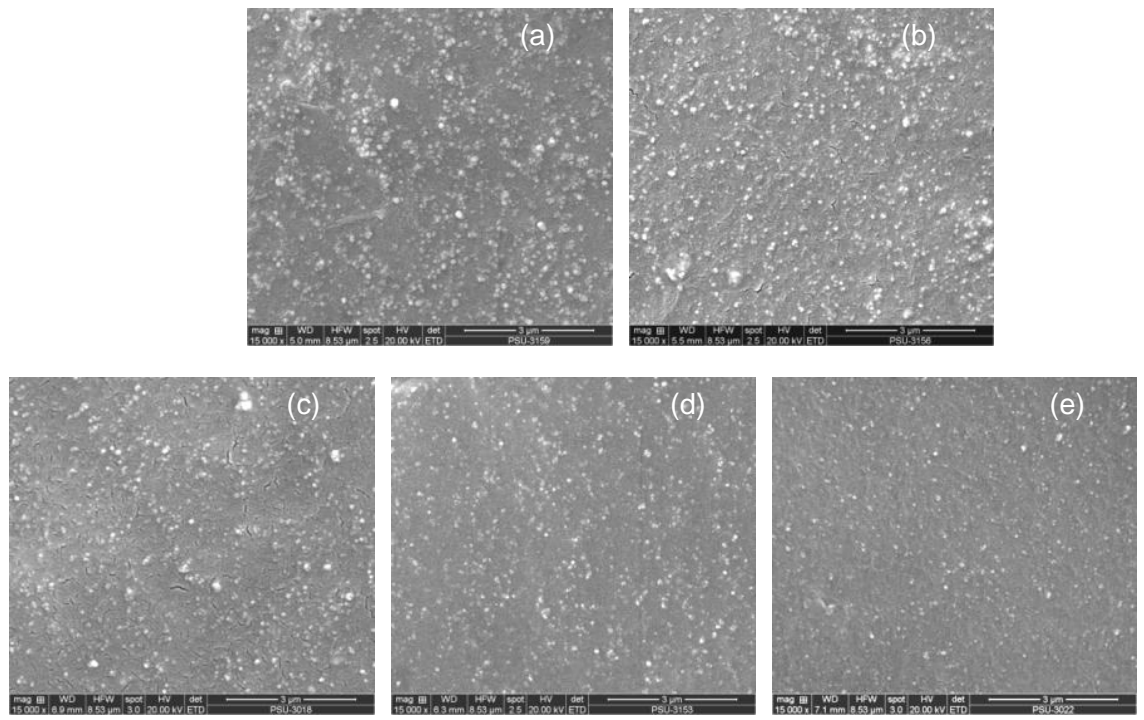

Figure 3.14 SEM micrographs of silica-filled NR vulcanizates with various types of compatibilizers at 15000x magnification; without compatibilizer (a), ENR-10 7.5 phr (b), ENR-38 7.5 phr (c), ENR-51 7.5 phr (d) and TESPT (e).

The silica dispersion level of filled-NR vulcanizates without any compatibilizer and with ENR-10 7.5 phr looks similar and poorer than that of the other compounds, as 
aggregates/agglomerates can clearly be observed. The addition of ENR-38 and ENR-51 at $7.5 \mathrm{phr}$ into the silica-filled compounds visibly shows an improvement of silica dispersion as shown in Figures 3.14c-d, respectively. This improved silica dispersion again supports the occurrence of interactions and/or reaction between silanol groups on the silica surface and epoxy groups of ENR, according to the mechanism proposed in Scheme 3.1 and 3.2, leading to reduction of polar $\mathrm{Si}-\mathrm{OH}$ groups, as also evidenced in the FTIR spectra Figure 3.13(a). The silica-filled NR with TESPT silane coupling agent (Figure 3.14(e)) shows the best silica distribution and dispersion which is in accordance with its superior properties.

\subsection{Conclusions}

Epoxidized natural rubber (ENR) can be used as compatibilizer in silica-filled natural rubber, as it results in improved filler-rubber interaction, silica dispersion and tensile strength. Increase of ENR content and mole\% of epoxide groups increases the interactions between silica and rubber, and decreases filler-filler interaction, as reflected by the lower Mooney viscosity, Payne effect, flocculation rate constant, filler networking factor, and the higher filler-rubber interaction parameter, chemically bound rubber, tensile strength and silica dispersion level. With respect to tire performance, the presence of ENR-38 and ENR-51 as compatibilizer improves wet grip but negatively affects rolling resistance. The addition of ENR-51 at $7.5 \mathrm{phr}$ shows the best overall properties, however still somewhat lower when compared to a compound with TESPT silane as coupling agent.

\subsection{References}

1. O. Seyvet, P. Nevard, J. Appl. Polym. Sci., 78, 1130 (2000).

2. A. Das, S.C. Debnath, D. De, D.K. Basu, J. Appl. Polym. Sci., 93, 196 (2004).

3. S.-S. Choi, J. Appl. Polym. Sci., 79, 1127 (2001).

4. J.-B. Donnet, Rubber Chem. Technol., 71, 323 (1998).

5. J.H. Bachmann, J.W. Sellers, M.P. Wagner, R.F. Wolf, Rubber Chem. Technol., 32, 1286 (1959).

6. H. Ismail, H.H. Chia, Eur. Polym. J., 34, 1857 (1998).

7. H. Ismail, A. Rusli, A.A. Rashid, Polym. Test., 24, 856 (2005).

8. A.K. Manna, A.K. Bhattacharyya, P.P. De, D.K. Tripathy, S.K. De, D.G. Peiffer, Polymer, 39, 7113 (1998). 
9. N. Suzuki, M Ito, S. Ono, J. Appl. Polym. Sci., 95, 74, (2005).

10. A.R. Payne, Rubber Plast. Age, 42, 963 (1961).

11. M.M. Jacobi, M.V. Braum, T.L.A.C. Rocha, R.H. Schuster, Kautsch. Gummi Kunstst., 60, 460 (2007).

12. T.L.A.C. Rocha, R.H. Schuster, M.M. Jacobi, D. Samios, Kautsch. Gummi Kunstst., 57, 656 (2004).

13. I.R. Gelling, M. Porter, "Natural Rubber Science and Technology", Oxford University Press, Oxford, 1988.

14. A.V. Chapman, In: $24^{\text {th }}$ International H.F. Mark-Symposium on Advances in the Field of Elastomers \& Thermoplastic Elastomers, Vienna, Austria, 2007. p. 15.

15. F. Cataldo, Macromol. Mater. Eng., 287, 348 (2002).

16. Y.Y. Luo, Y.Q. Wang, J.P. Zhong, C.Z. He, Y.Z. Li, Z. Peng, J. Inorg. Organomet. Polym., 21, 777, (2011).

17. S.H. Sandstrom, T.J. Segatta, J.J.A. Verthe, US Patent 5396940, to The Goodyear Tire \& Rubber Company, March 14, 1995.

18. S. Schaal, A.Y. Coran, S.K. Mowdood, US Patent 6482884 B1, to Pirelli Pneumatici S.p.A., November 19, 2002.

19. C.J. Lin, W.L. Hergenrother, US Patent 6845797 B2, to Bridgestone Corporation, January 25, 2005.

20. C. Kantala, E. Wimolmala, C. Sirisinha, N. Sombatsompop, Polym. Adv. Technol., 20, 448 (2009).

21. K.M. George, J.K. Varkey, K.T. Thomas, N.M. Mathew, J. Appl. Polym. Sci., 85, 292 (2002).

22. K.M. George, J.K. Varkey, B. George, S. Joseph, K.T. Thomas, N.M. Mathew, Kautsch. Gummi Kunstst., 59, 544 (2006).

23. P.L. Teh, Z.A. Mohd Ishak, A.S. Hashim, J. Karger-Kocsis, U.S. Ishiaku, J. Appl. Polym. Sci., 94, 2438 (2004).

24. P.L. Teh, Z.A. Mohd Ishak, A.S. Hashim, J. Karger-Kocsis, U.S. Ishiaku, Eur. Polym. J., 40, 2513 (2004).

25. C. Nakason, A. Kaesaman, T. Wongkul, S. Kiatkamjornwong, Plast. Rubb. Compos., 30, 154 (2001).

26. I.R. Gelling, Rubber Chem. Technol., 58, 86 (1985).

27. L. Guy, S. Daudey, P. Cochet, Y. Bomal, Kautsch. Gummi Kunstst., 62, 383 (2009).

28. S. Mihara, R.N. Datta, J.W.M. Noordermeer, Rubber Chem. Technol., 82, 524 (2009). 
29. J.A. Ayala, W.M. Hess, G.A. Joyce, F.D. Kistler, Rubber Chem. Technol., 66, 772 (1993).

30. S. Wolff, M.J. Wang, E.H. Tan, Rubber Chem. Technol., 66, 163 (1993).

31. S.C. Ng, K.K. Chee, Rubber Chem. Technol., 62, 585 (1989).

32. T.L.A.C. Rocha, C. Rosca, J. Ziegler, R.H. Schuster, Kautsch. Gummi Kunstst., 58, 22 (2005).

33. A.K. Manna, P.P. De, D.K. Tripathy, S.K. De, D.G. Peiffer, J. Appl. Polym. Sci., 74, 389 (1999).

34. H.-D. Luginsland, J. Frohlich, A. Wehmeier, Rubber Chem. Technol., 75, 563 (2002).

35. G.A. Böhm, W. Tomaszewski, W. Cole, T. Hogan, Polymer, 51, 2057 (2010).

36. A.J. Manna, P.P De, D.K. Tripathy, J. Appl. Polym. Sci., 84, 2171 (2002).

37. S. Bandyopadhyay, P.P. De, D.K. Tripathy, S.K. De, Rubber Chem. Technol., 69, 637 (1996).

38. J.W. ten Brinke, S.C. Debnath, L.A.E.M. Reuvekamp, J.W.M. Noordermeer, Compos. Sci. Technol., 63, 1165 (2003).

39. W. Kaewsakul, K. Sahakaro, W.K. Dierkes, J.W.M. Noordermeer, Rubber Chem. Technol., 85, 277 (2012).

40. J.T. Sakdapipanich, J. Biosci. Bioeng., 103, 287 (2007).

41. S.S. Sarkawi, W.K. Dierkes, J.W.M. Noordermeer, Eur. Polym. J., 49, 3199 (2013).

42. S. Wolff, M.J. Wang, E.H. Tan, Kautsch. Gummi Kunstst., 47, 873 (1994).

43. M.M. Jayasuriya, D.J. Hourston, J. Appl. Polym. Sci., 112, 3217 (2009).

44. R. Rajasekar, K. Pal, G. Heinrich, A. Das, C.K. Das, Mater. Design, 30, 3839 (2009).

45. S. Maiti, S.K. De, A.K. Bhowmick, Rubber Chem. Technol., 65, 293 (1992).

46. P. Saramolee, K. Sahakaro, N. Lopattananon, W.K. Dierkes, J.W.M. Noordermeer, Rubber Chem. Technol., in press (2014). doi: http://dx.doi.org/10.5254/rct.13.86970.

47. C.G. Robertson, C.M. Roland, Rubber Chem. Technol., 81, 506 (2008).

48. Y. Li, M.J. Wang, T. Zhang, Rubber Chem. Technol., 67, 693 (1994).

49. M.K. Kralevich, J.L. Koenig, Rubber Chem. Technol., 71, 300 (1997).

50. A. Bertora, M. Castellano, E. Marsano, M. Alessi, L. Conzatti, P. Stagnaro, G. Colucci, A. Priola, A. Turturro, Macromol. Mater. Eng., 296, 455 (2011).

51. L.T. Zhuravlev, Colloids Surf. A: Physicochem. Eng. Aspects, 173, 1 (2000). 


\section{Silica-Reinforced Natural Rubber Compounds Compatibilized by ENR in combination with TESPT and Sulfur Compensation}

In the previous study as described in Chapter 3, the use of ENR containing 51 mol\% epoxide groups (ENR-51) as a compatibilizer without bis-(triethoxysilylpropyl) tetrasulfide (TESPT) coupling agent was optimized at 7.5 phr, giving properties which were still somewhat interior to the compound with TESPT. In this chapter, ENR and TESPT are cooperatively used to improve the properties of silica-filled NR. By using 7.5 phr of ENR-51 with varying amounts of TESPT in a range of 2 to 5 wt\% relative to the silica, the properties of compounds are compared to those with optimum TESPT content, i.e. 8.6 wt\% relative to the silica, and without TESPT. The addition of TESPT to the ENR51 compatibilized silica-filled NR compound has no effect on Mooney viscosity but lowers the Payne effect to the same level as that of the silica/TESPT compound. It significantly decreases both scorch and optimum cure times, and increases the tensile strength to match the property of the optimized silica/TESPT system while maintains the elongation at break. Furthermore, the tan $\delta$ at $60^{\circ} \mathrm{C}$ is lowered by the addition of TESPT and extra sulfur when compared to the use of ENR-51 alone as compatibilizer, while the tan $\delta$ at $0^{\circ} \mathrm{C}$ is maintained. Silica dispersion in the NR matrix and increased interactions within the material by use of the compatibilizer are evidenced by SEM analysis. This work demonstrates that the use of ENR as compatibilizer already enhances the properties of silica-filled NR compounds, and that such properties can further be improved by adding TESPT at half or lower amount of TESPT than normally needed for silica-filled compounds, and can be further improved by sulfur compensation relative to the reference compound with TESPT alone. The combination of ENR, TESPT and sulfur compensation presents itself as a better option than TESPT alone, for tires where skid-resistance is of prime importance; e.g. winter tires.

Part of this chapter was published in Advanced Materials Research, 844, 272(2014). 


\subsection{Introduction}

Silica-filled compounds offer a better wet traction and lower rolling resistance for passenger tire tread compounds, compared to carbon black-filled counterparts. ${ }^{[1,2]}$ However, silica mixing requires longer mixing time and higher mixing temperatures than carbon black. Moreover, silica-filled hydrocarbon rubber compounds without any coupling agent as compatibilizer suffer from poor silica dispersion due to strong filler-filler interactions and thus result in inferior mechanical properties. To enhance the compatibility between silica and non-polar rubbers, a silane coupling agent or polar rubber such as acrylonitrile-butadiene rubber $(\mathrm{NBR})^{[3]}$, polychloroprene rubber $(\mathrm{CR})^{[4]}$ and epoxidized natural rubber $(E N R)^{[5]}$ can be used as a compatibilizer. The most widely used silane coupling agent in silica-filled compounds cured with a sulfur system is bis(triethoxysilylpropyl)tetrasulfide (TESPT). It has long been known that this sulfurcontaining silane coupling agent also acts as a sulfur donor to promote crosslinking in the compound. Under optimal mixing conditions, the use of TESPT effectively reduces fillerfiller interaction and enhances silica-rubber interaction. ${ }^{[6,7]}$ The silica/silane combination leads to a good silica dispersion and significant improvement in mechanical and dynamical properties due to the rubber-silica bonds bridged by TESPT. ${ }^{[8]}$

Epoxidized natural rubber shows a higher polarity than original natural rubber (NR) due to the epoxy groups in its structure. Its viscosity and polarity increases with increasing epoxy content. ${ }^{[9]}$ ENR has been used as a compatibilizer in filled systems such as NR-organoclay nanocomposites ${ }^{[10,11]}$ in which the use of ENR improved filler-rubber interaction and filler dispersion in the matrix. The improved compatibility between filler and rubber subsequently improved physical and mechanical properties of the nanocomposites. $^{[10,11]}$ Silica-filled ENR shows significantly improved mechanical properties when compared to a silica-filled NR counterpart without silane, because of an interaction of ENR and silica through hydrogen bonds. ${ }^{[12]}$ In addition, chemical bonding between epoxy groups of ENR and silanol groups of silica can be promoted during molding at high temperatures ${ }^{[3]}$ or mixing at $150-170^{\circ} \mathrm{C}^{[13]}$ At high temperature, epoxygroups of ENR could break down to become hydroxyl groups and to bond to the silica surface through $\mathrm{Si}-\mathrm{O}-\mathrm{C}$ bonds. ${ }^{[5,13]}$

As demonstrated in Chapter 3, the addition of ENR as compatibilizer in silicafilled NR compounds enhanced both processing and vulcanizate properties. The Payne effect was reduced and filler-rubber interaction was promoted leading to the improvement of mechanical properties. However, the properties were still lower compared to the reference compound that employed TESPT as coupling agent. One of the reasons can be 
attributed to the influence of network formation within the material. The TESPT containing compounds obtain a contribution from sulfur that is released from TESPT and so have a higher network density. A cooperative effect of epoxide groups in ENR and TESPT on reinforcing efficiency of silica-filled ENR compounds was recently reported. ${ }^{[14]}$ The use of epoxidized low molecular weight natural rubber (ELMWNR) as compatibilizer in silica-filled NR compounds reduced the Payne effect and improved the mechanical properties when compared to an uncompatibilized system. ${ }^{[15]}$ In addition, the effect of extra sulfur added into the silica-filled NR compounds compatibilized with ELMWNR was reported to enhance modulus and tensile strength as well as to reduce the loss tangent at $60^{\circ} \mathrm{C} .^{[16]}$ Herein, the amount of extra sulfur was calculated to compensate for the sulfur in the TESPT molecules.

In Chapter 3, the use of 7.5 phr of ENR-51 as compatibilizer showed the best overall properties of the silica-filled NR compounds, but still inferior to those of the reference compound with TESPT. This chapter is therefore to investigate the use of 7.5 phr of ENR-51 in combination with TESPT at varying amounts from 2 to $5 \mathrm{wt} \%$ relative to silica, without and with sulfur compensation. The compensation is applied by adding elemental sulfur into the compound to adjust the total sulfur content, equal to the sulfur contained in the reference compound with $4.7 \mathrm{phr}$ of TESPT.

\subsection{Experimental}

\subsubsection{Materials and compound preparation}

In this chapter, the materials used for compounding are as described in Chapter 3. The compound recipes are shown in Table 4.1, and the same two-step mixing procedure, as described in Table 3.2, is employed for compound preparation. The first mixing step was carried out using an internal mixer (Brabender Plasticorder 350s) with an initial mixer temperature setting of $110^{\circ} \mathrm{C}$, rotor speed of $60 \mathrm{rpm}$ to obtain the optimized dump temperature in the range of $135-150^{\circ} \mathrm{C} .^{[6]}$ The second step of curative mixing was carried out using a two-roll mill for 5 mins. All elemental sulfur was added in this second mixing step. 
Table 4.1 Compound formulations.

\begin{tabular}{lcccc}
\hline \multirow{2}{*}{ Ingredients } & \multicolumn{4}{c}{ Parts per hundred parts of rubber (phr) } \\
\cline { 2 - 5 } & None & TESPT & ENR+TESPT & ENR+TESPT+S \\
\hline RSS3, Natural Rubber & 100.0 & 100.0 & 92.5 & 92.5 \\
ENR-51 & - & - & 7.5 & 7.5 \\
TESPT & - & $4.7^{\star}$ & $1.1-2.7^{\star *}$ & $1.1-2.7$ \\
Zeosil 1165MP & 55.0 & 55.0 & 55.0 & 55.0 \\
TDAE oil & 8.0 & 8.0 & 8.0 & 8.0 \\
ZnO & 3.0 & 3.0 & 3.0 & 3.0 \\
TMQ & 1.0 & 1.0 & 1.0 & 1.0 \\
Stearic acid & 1.0 & 1.0 & 1.0 & 1.0 \\
DPG & 1.0 & 1.0 & 1.0 & 1.0 \\
CBS & 1.5 & 1.5 & 1.5 & 1.5 \\
Sulfur & 1.5 & 1.5 & 1.5 & $2.3-1.9^{\star \star *}$ \\
\hline
\end{tabular}

Remarks: * TESPT $4.7 \mathrm{phr}$ equal to $8.6 \mathrm{wt} \%$ relative to silica; ${ }^{* *}$ TESPT 1.1-2.7 phr are equal to 2-5 wt $\%$ relative to silica; ${ }^{* * *}$ sulfur contents were adjusted to compensate for the sulfur in the TESPT molecules by taking the compound with $4.7 \mathrm{phr}$ of TESPT as reference.

\subsubsection{Testing of compound and vulcanizate properties}

Mooney viscosity, Payne effect, flocculation rate constant, bound rubber content, cure characteristics, tensile and dynamic mechanical properties of the silica-filled NR compounds were investigated following the methods and using the equipments as described in Chapter 3. For dynamic mechanical properties, the DMA (Metravib Viscoanalyzer VA 2000, Metravib R.D.S.) was operated in tension mode under the same conditions as applied in Chapter 3.

\subsubsection{SEM analysis of silica-filled NR vulcanizates}

SEM analysis was performed by using two different sets of specimens. First, compounds containing $30 \mathrm{phr}$ of silica, without compatibilizer, with ENR-51 $7.5 \mathrm{phr}$, with ENR-51 $7.5 \mathrm{phr}$ and TESPT $4 \mathrm{wt} \%$ relative to silica, and with TESPT $8.6 \mathrm{wt} \%$ relative to silica, were prepared using the same formulation as shown in Table 4.1, except the lower amount of silica. The compounds were vulcanized to their optimum cure times to produce a thin sheet of 1-2 mm thickness, immersed in liquid nitrogen, and cryogenically cracked. The newly cracked surface was gold-coated before being analyzed by SEM (Quanta 400, FEI). The second set of vulcanized specimens was taken from tensile-fractured test pieces which consisted of 55 phr of silica, i.e. at normal silica content used in this study. The tensile-fractured surface was also coated with gold prior to being analyzed. 


\subsection{Results and discussion}

\subsubsection{Mooney viscosity, Payne effect and flocculation tendency}

Figure 4.1 shows the Mooney viscosities of the silica-reinforced NR compounds with ENR-51 as compatibilizer in combination with varying contents of TESPT, with and without extra sulfur, in comparison with that of the compounds with no compatibilizer at all and with only $4.7 \mathrm{phr}$ of TESPT as reference.

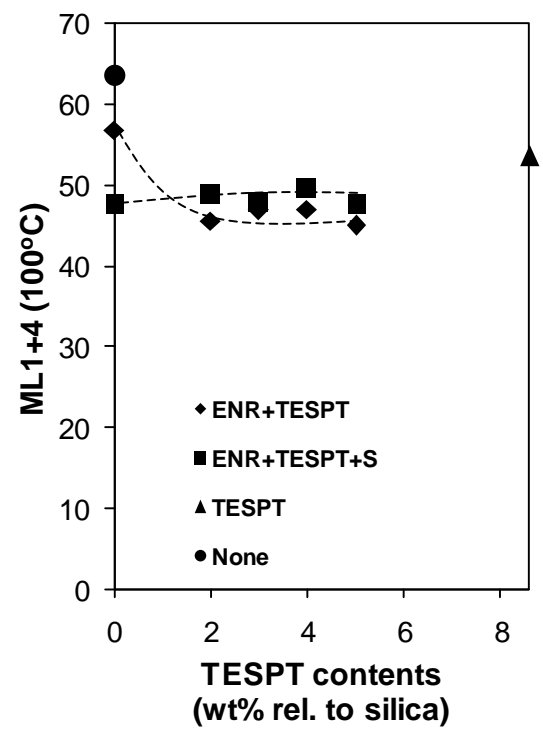

Figure 4.1 Mooney viscosity of silica-filled NR compounds with $7.5 \mathrm{phr}$ of ENR-51 as compatibilizer in combination with TESPT with and without sulfur compensation.

Without extra sulfur, the incorporation of $2 \mathrm{wt} \%$ TESPT relative to silica into the silica-reinforced NR compounds leads to a decrease of Mooney viscosity, suggesting a further improvement of silica dispersion in the NR matrix. But a further increase of TESPT contents shows no additional effect on this property. The compounds with extra sulfur show no change in Mooney viscosity with increasing silane content and the values are marginally higher than that of the silica-filled NR compounds with no extra sulfur. All of the compounds with ENR-51/TESPT combinations, either with or without extra sulfur, show lower Mooney viscosities when compared to the reference compound with $4.7 \mathrm{phr}$ TESPT. The lower Mooney viscosity may be attributed to a better filler distribution causing smaller silica aggregates and less resistance to flow. As the sulfur was added in the second step 
of mixing on a two-roll mill, a premature crosslinking reaction is unlikely to take place like in the case of addition of extra sulfur into the internal mixer at high temperature. ${ }^{[7]}$ Nevertheless, the compounds with TESPT and extra sulfur show a slightly higher Mooney viscosity compared to the mixes with lower sulfur content. It should be noted that, in the absence of TESPT, the ENR-compatibilized compound with higher sulfur content shows a lower Mooney viscosity.
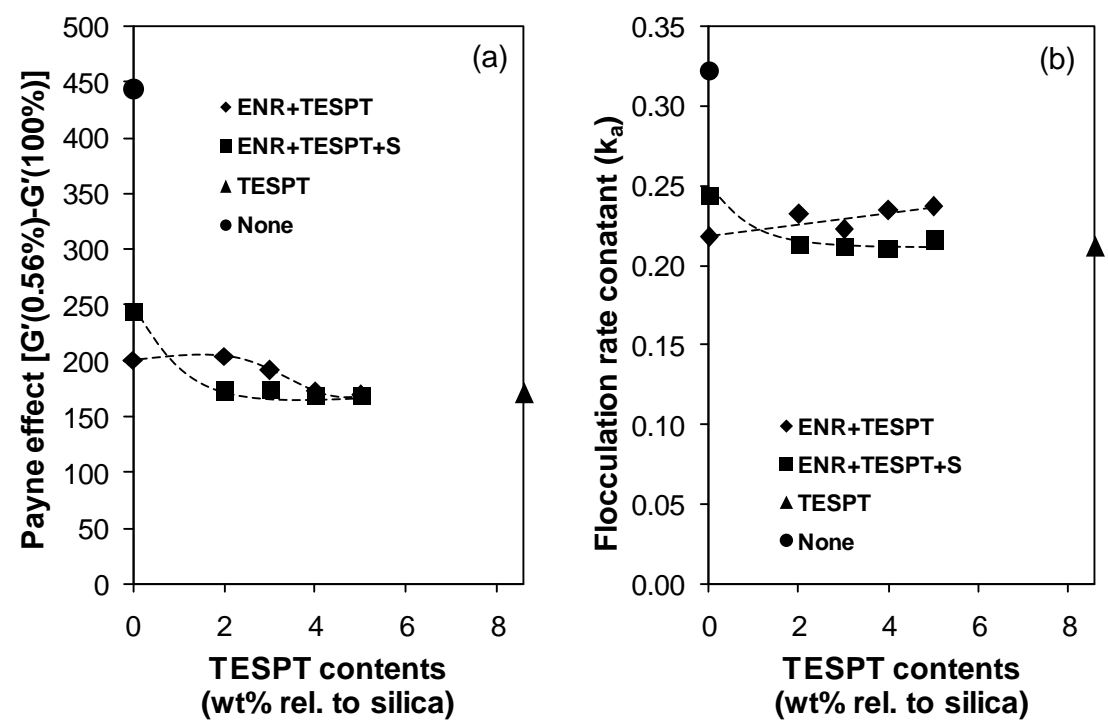

Figure 4.2 Payne effect (a) and flocculation rate constant (b) of silica-filled NR compounds with 7.5 phr of ENR-51 as compatibilizer in combination with TESPT with and without sulfur compensation.

The filler-filler interaction or Payne effect of the silica-filled NR compounds is shown in Figure 4.2 (a). The addition of 7.5 phr of ENR-51 already remarkably decreases the filler-filler interactions in the silica-filled NR compounds when compared to the noncompatibilized one. This is due to interaction between silanol groups on the silica surface and epoxy groups of ENR, as previously described in Chapter 3. The use of extra sulfur in the silica-filled NR compounds with ENR-51 as compatibilizer results in a somewhat higher Payne effect compared to the compound with normal sulfur content. This may be due to their lower Mooney viscosities: Figure 4.1, that cause a tendency of silica reagglomeration, i.e. flocculation, which is in agreement with the flocculation rate constant in Figure 4.2 (b). The flocculation process is the reformation of silica-silica interaction in the compound under heat treatment to regenerate a too rigid silica network. ${ }^{[17]}$ The 
flocculation behavior of silica-filled NR compounds containing ENR-51 as compatiblilizer in combination with TESPT and extra sulfur shows the same trend as the Payne effect.

The Payne effect: Figure 4.2 (a) and flocculation rate constant: Figure 4.2 (b) of the silica-filled NR compounds with 2 wt\% TESPT relative to silica and extra sulfur decrease to the same level as that of the reference compound with TESPT only. This further reduction of filler-filler interaction by the addition of TESPT suggests that even though epoxy-groups of ENR interact with the silanol-groups on the silica surfaces, there are still free silanol-groups present in the system. So, the use of just small amounts of TESPT can further decrease the filler-filler interactions through the silanization reaction of ethoxy-groups and silanol-groups as shown in Scheme 4.1. Without TESPT, the compound with extra sulfur which has a lower Mooney viscosity shows a higher Payne effect and higher flocculation rate constant. But in the presence of both TESPT and extra sulfur, the compounds that have higher Mooney viscosities exhibit lower Payne effects as well as lower flocculation rate constants, and the values are similar to that of the reference silica/TESPT system. The results suggest an influence of Mooney viscosity on flocculation rate constant which in turn relates to the Payne effect.

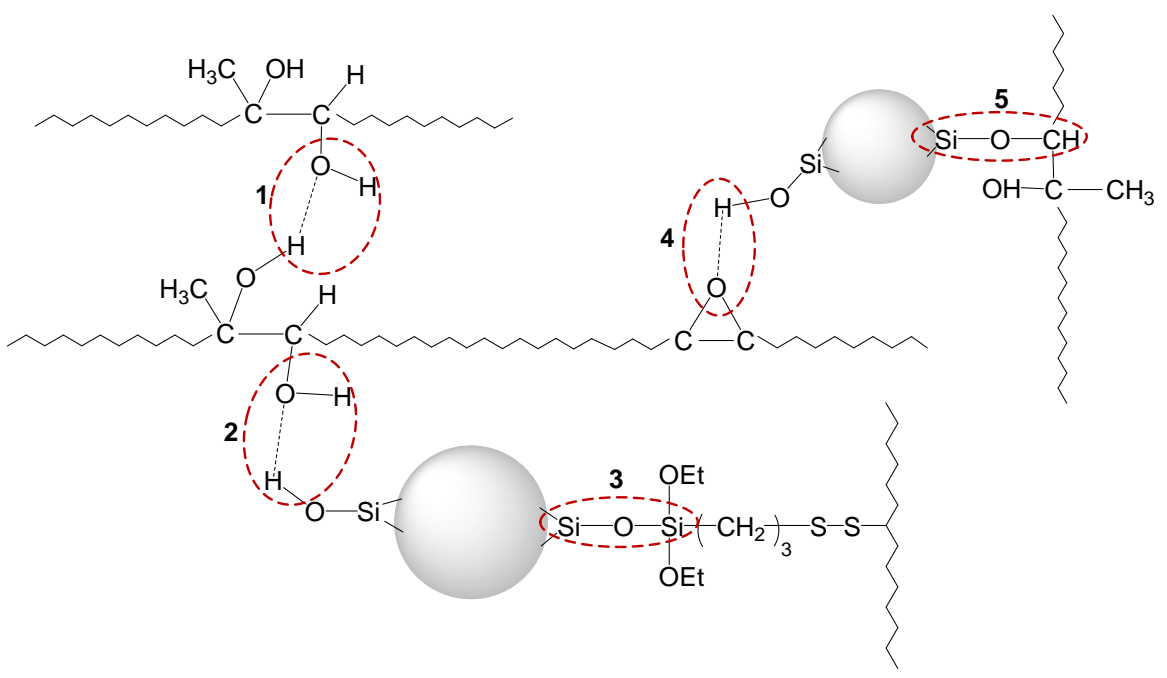

Scheme 4.1 Various possible interactions and bonding in the silica-filled NR compounds compatibilizied with ENR in combination with TESPT.

There are several possible interactions and/ or reactions within the system including: (1) interaction between hydroxyl-groups of ring-opened ENR; (2) interaction between hydroxyl-group of ring-opened ENR with silanol group of silica ${ }^{[18]}$; (3) bonding 
between silica and rubber molecules via silane molecules; (4) interaction between epoxy and silanol groups; and (5) bonding between silanol and hydroxyl groups, as shown and numbered in Scheme 4.1. The interactions/reactions between epoxy- or hydroxyl-groups of the ENR and silanol groups of the silica contribute to the decreased silica-silica interactions in the system.

\subsubsection{Filler-rubber interactions}

Silica particles and aggregates commonly form a filler-filler network to their neighbors due to the polar silanol groups on the surface ${ }^{[19]}$ causing a poor filler-rubber interaction which is a key parameter for reinforcement. Bound rubber content can optionally be used as an indication for filler-rubber interactions ${ }^{[20]}$ in the silica-reinforced rubber compounds. The results of silica-filled NR compounds compatibilized with ENRs and TESPT are shown in Figure 4.3. The test was performed using the non-productive compounds, i.e. the compounds without curatives, collected after the first mixing step. Ammonia treatment was employed during the bound rubber test to destroy weak physical interactions, so that the chemically bound rubber content could be determined. ${ }^{[20]}$ The silica-filled compound without compatibilizer shows the lowest chemically bound rubber content because of high filler-filler interactions through hydrogen bonding between silica aggregates and the poor interactions between silica and rubber due to their difference in polarity. The reference silica-filled NR compound with $4.7 \mathrm{phr}$ TESPT gives the highest chemically bound rubber content which is distinctively higher than the value for the noncompatibilized compound, due to a good interfacial interaction via the silanization reaction of silanol-groups of silica and the ethoxy-groups of the silane. The ENR-compatibilized silica-filled NR compounds also show an improvement in bound rubber content compared to the compound without compatibilizer, due to the interactions/reactions between epoxyand silanol-groups. The chemically bound rubber contents increase slightly with the addition of TESPT and with increasing TESPT contents, as a result of an increase of chemical bonds between silica and the rubber matrix through the silane bridge. ${ }^{[21]}$ However, the level of chemically bound rubber does not reach the value observed for 4.7 phr TESPT. 


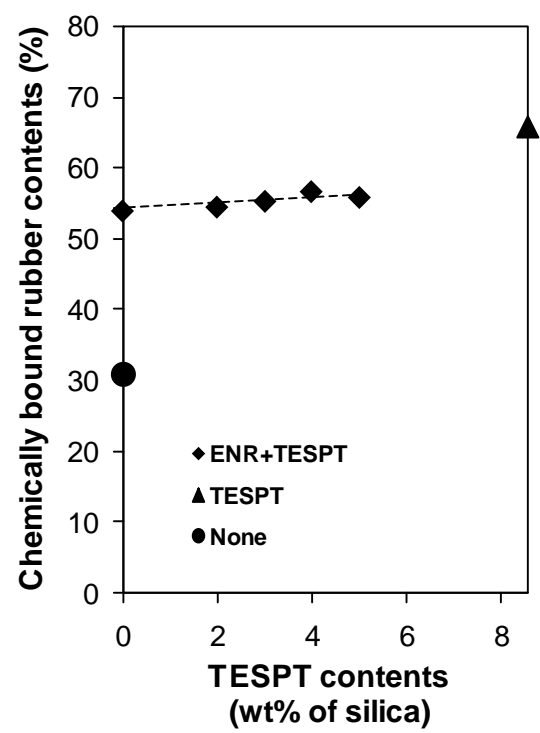

Figure 4.3 Chemically bound rubber content of silica-filled NR masterbatches with $7.5 \mathrm{phr}$ of ENR-51 as compatibilizer in combination with TESPT.

\subsubsection{Cure characteristics}
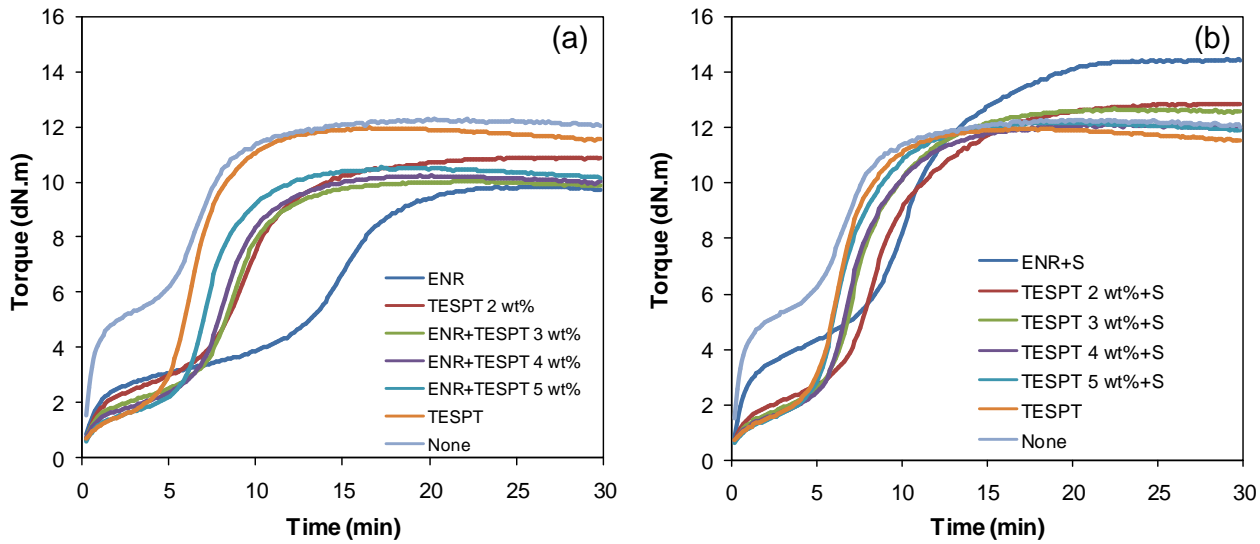

Figure 4.4 Cure curves of silica-filled NR compounds with $7.5 \mathrm{phr}$ of ENR-51 as compatibilizer and varying contents of TESPT without sulfur compensation (a) and with sulfur compensation (b).

Cure curves of the silica-filled compounds with different types of compatibilizer are shown in Figures 4.4(a) and (b) for the compounds without and with extra sulfur, 
respectively. The silica-filled NR compound without any compatibilizers shows a clear increase of rheometer torque at the beginning of the cure curve, prior to the vulcanization reaction, which can be attributed to flocculation of silica aggregates that is accelerated by heat, resulting in the highest minimum and maximum torques as seen in Figure 4.4(a). The use of the optimal TESPT content of $4.7 \mathrm{phr}$ diminishes the flocculation phenomenon in the silica-filled compound due to strong interactions between silica and rubber via the silane so that the silica surface become less polar. The addition of only ENR-51 at $7.5 \mathrm{phr}$ as compatibilizer significantly decreases filler flocculation, but the scorch and cure times are largely prolonged due to the presence of highly polar epoxy groups that may interfere with the curatives and furthermore, some residual acid in the ENR may retard the cure. This compound also shows the lowest maximum torque indicating the least overall network crosslink density.

Silica has a hydrophilic character ${ }^{[22]}$ and can easily form a strong interaction with polar functional groups or polar rubbers like ENR. ${ }^{[5]}$ The addition of ENR-51 to a silicafilled NR compound introduces interactions between the epoxy-groups of the ENR and silanol-groups of the silica to some extent, but there will remain some free polar functional groups that may interact with polar ingredients generated during the vulcanization reaction, such as sulfurating intermediates, and result in longer scorch and cure times. ${ }^{[23,24]}$ The use of TESPT in combination with ENR-51 for compatibilizing the silicafilled NR compounds effectively decreases silica flocculation and the cure behavior is improved with increasing TESPT concentrations due to a higher degree of silica hydrophobation and increase of silica-rubber interaction. ${ }^{[25]}$ However, the maximum cure torques and cure rate indices are still lower when compared to the use of the optimal content of TESPT of $4.7 \mathrm{phr}$.

The addition of extra sulfur into the compounds definitely has a positive effect on cure behavior due to the presence of a higher content of curatives. The compound that is compatibilized by ENR alone in the presence of extra sulfur shows still lower flocculation compared with the non-compatibilized one, but a remarkably higher flocculation tendency than the compound with TESPT, as seen in Figure 4.4(b). The addition of extra sulfur into the ENR/TESPT-compatibilized silica-filled compounds increases the maximum cure torques of the compounds compared to the results in Figure 4.4(a) due to the increase of crosslink density in the vulcanizates. The flocculation phenomenon of the ENRcompatibilized compound with extra sulfur is in accordance with the higher Payne effect: Figure 4.2 (a) and higher flocculation rate constant: Figure 4.2 (b). The presence of ENR51 alone is apparently not sufficient to interact with the silanol groups of the silica, to suppress the flocculation and enhance the cure characteristics. The use of TESPT in 
smaller amounts than in the referent formulation can overcome the problems. In the case of the compounds with ENR-51 and extra sulfur, due to a reduction of compound viscosity when sulfur was additionally added (Figure 4.1), an easier condition for filler flocculation was generated as the silica aggregates could migrate faster in the lower viscosity matrix.
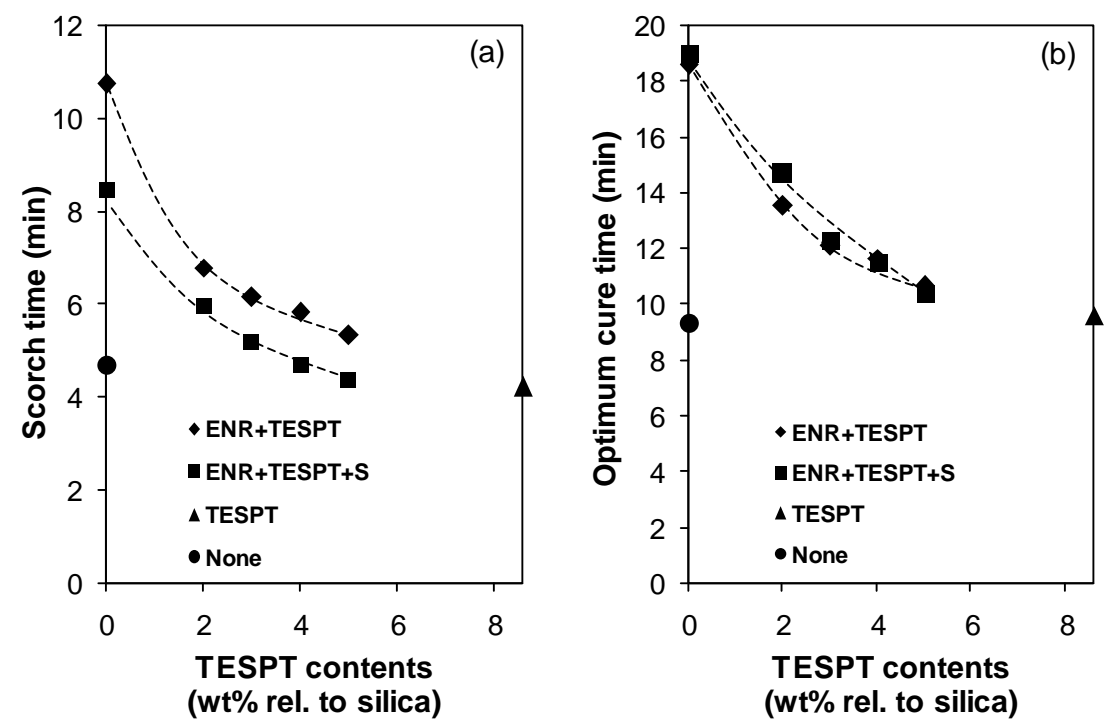

Figure 4.5 Scorch and cure times of silica-filled NR compounds with ENR-51 at 7.5 phr, in combination with TESPT silane coupling agent as compatibilizers, without and with sulfur compensation.

Scorch $\left(t_{s 1}\right)$ and optimum cure times $\left(t_{c}(90)\right)$ of the silica-filled NR compounds with 7.5 phr of ENR-51 in combination with TESPT without and with extra sulfur are shown in Figures 4.5(a) and 4.5(b), respectively. These figures help to give a clear picture of the changes in scorch and cure times between the various compounds, according to the cure curves as shown in Figures 4.4(a) and 4.4(b). The use of ENR-51 alone as compatibilizer in the silica-filled NR compound shows the longest $t_{s 1}$ and $t_{c}(90)$ due to the remaining free silanol groups and polar epoxide groups that can adsorb polar curatives and so retard the vulcanization reaction as described previously. Residual acid left in the ENR may also take part in the cure retardation. The incorporation of TESPT in the silica-filled compound helps to reduce the remaining polar moieties through the silanization reaction. In addition, TESPT acts as a sulfur donor for the vulcanization reaction ${ }^{[7]}$, leading to a faster cure time compared to the silica-filled NR with ENR alone as compatibilizer. The addition of extra sulfur in the systems results in shorter scorch times with increasing TESPT 
concentrations, compared to the compounds with normal sulfur content, due to the presence of a higher concentration of curatives that can generate crosslinks. But the compounds with different sulfur contents show mutually more or less the same optimum cure times, which decrease with increasing TESPT loadings. Interestingly, the noncompatibilized compound show similar scorch and cure times to that of the silica/TESPT system despite the polar and acidic silanol groups. This might be attributed to the occurrence of the filler-filler network, as seen by its highest Payne effect and flocculation rate constant in Figure 4.2, that results in a smaller surface area and less available silanol groups. On the side, the TESPT-system has a good filler dispersion, the aggregate size is smaller, providing more surface area and free silanol groups to pay a role in the vulcanization reaction. With this competitive balance, the compounds with no compatibilizer and with optimal TESPT content show equal optimum cure times as shown in Figure 4.5(b).

\subsubsection{Tensile properties}
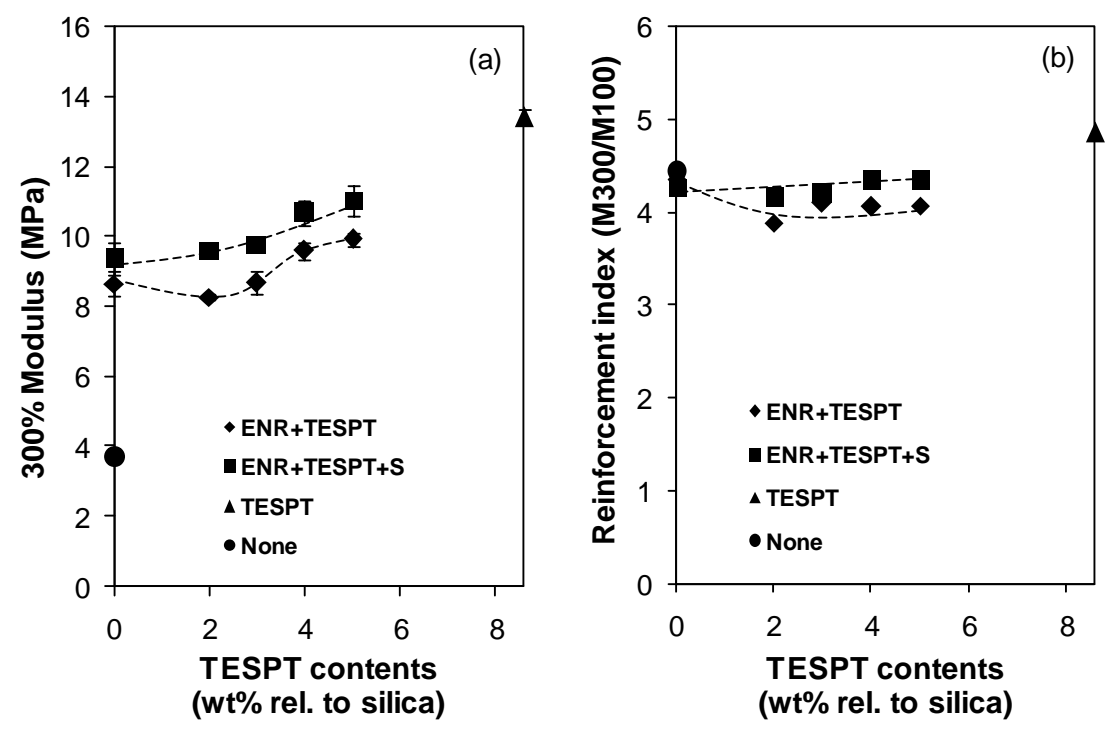

Figure $4.6300 \%$ Modulus and reinforcement index (M300/M100) of silica-filled NR compounds with 7.5 phr of ENR-51 in combination with TESPT without and with sulfur compensation.

The incorporation of TESPT without sulfur compensation clearly enhances the modulus at $300 \%$ elongation but the values of the reinforcement index of the silica-filled 
NR vulcanizates do not change much, as respectively shown in Figures 4.6(a) and (b). This is due to an increase of silica-rubber interactions/reactions in the system. The introduction of more sulfur to match the level of sulfur available in the reference TESPT/silica system significantly increases the tensile modulus of the vulcanizates because of an increased amount of crosslinking agent and consequently a higher crosslink density and more stiffness. With ENR/TESPT and extra sulfur, the vulcanizates still show a lower $300 \%$ modulus when compared to the compound with the optimal TESPT content of $4.7 \mathrm{phr}$. The reinforcement index (M300/M100) of the silica-filled NR vulcanizates is improved when TESPT and extra sulfur are added, however an increasing amount of TESPT has no clear effect on the reinforcement index of the vulcanizates. All of the compatibilized compounds show a remarkable improvement in modulus compared with the one without, but with little dependence on the TESPT-content.
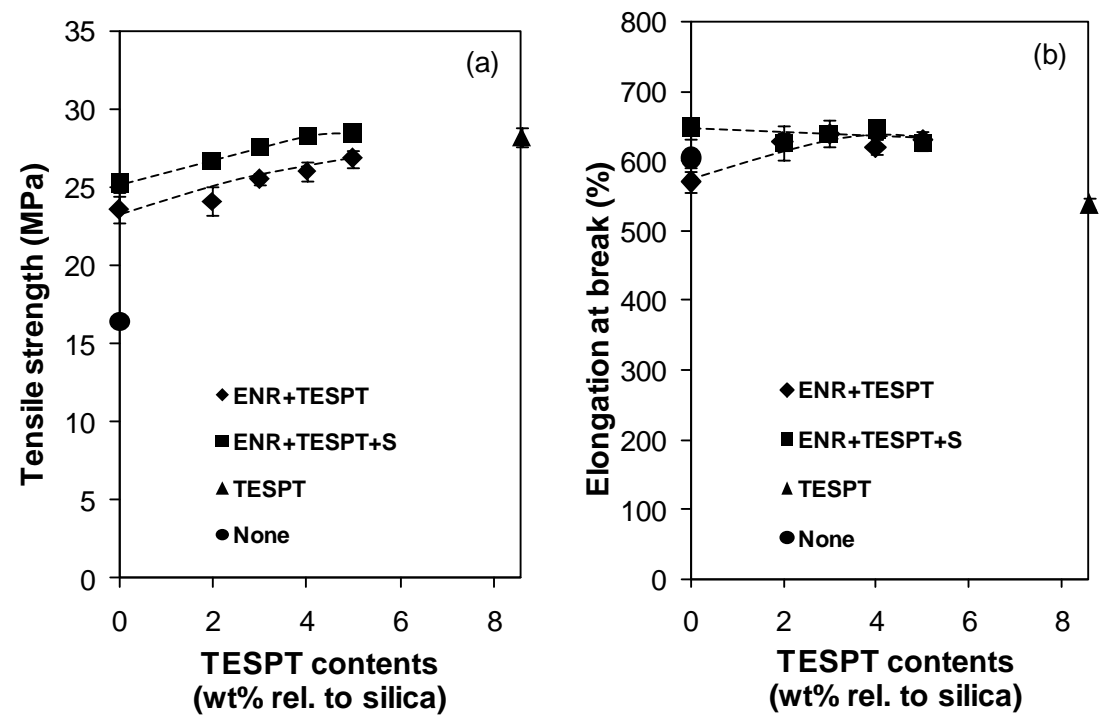

Figure 4.7 Tensile strength (a) and elongation at break (b) of silica-filled NR compounds with 7.5 phr of ENR-51 in combination with TESPT without and with sulfur compensation.

The tensile strength: Figure 4.7(a), of the vulcanizates is improved by adding TESPT on top of the ENR-51 as compatibilizer, and further enhanced by the addition of extra sulfur. The use of TESPT at $4 \mathrm{wt} \%$ relative to the silica with ENR-51 and extra sulfur gives the same level of tensile strength as that of the silica/TESPT filled reference compound, while the elongation at break remains more or less unchanged: Figure 4.7(b). In combination with the epoxy-groups that can interact and/or react with the silanol-groups 
of silica, the amount of TESPT needed to optimize the silica-filled compound properties can be reduced, compared to a conventional TESPT-silanized silica filled system. The different levels of modulus and tensile strength caused by the difference in sulfur contents because of the sulfur donation of TESPT, can be improved by introducing more crosslinks generated by extra elemental sulfur added.

\subsubsection{Dynamic mechanical properties}

Silica-filled NR-vulcanizates with various types of compatibilizers have different dynamic mechanical properties as shown in Figures 4.8-4.9. These properties are related to the viscoelastic behavior of the materials, which depend on several factors including polymer characteristics, reinforcing filler types and contents, process oil, crosslink density, etc. In tire technology, the viscoelastic properties of the rubbers are intrinsic characteristics that are related to tire performance, i.e. wet traction and rolling resistance. ${ }^{[26]}$
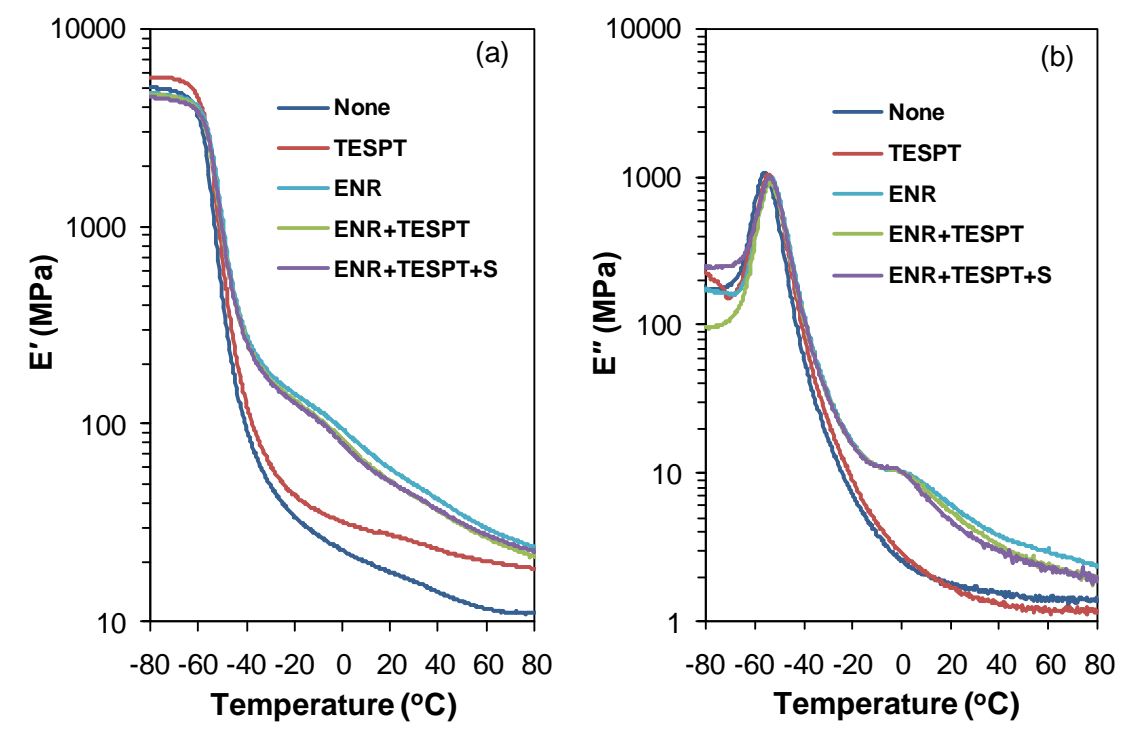

Figure 4.8 Storage modulus (a) and loss modulus (b) of silica-filled NR vulcanizates containing different compatibilizer types and combinations.

The storage modulus, loss modulus and $\tan \delta$ as functions of temperature of the silica-filled NR vulcanizates are shown in Figures 4.8(a), 4.8(b) and 4.9. In these figures, the results of the vulcanizates with 8.6 wt\% TESPT relative to silica, with 7.5 phr ENR-51, with 7.5 phr ENR-51 plus 4 wt\% TESPT relative to silica, and with 7.5 phr ENR-51 plus 4 
wt\% TESPT relative to silica plus extra sulfur, as compatibilizers, are comparatively displayed.

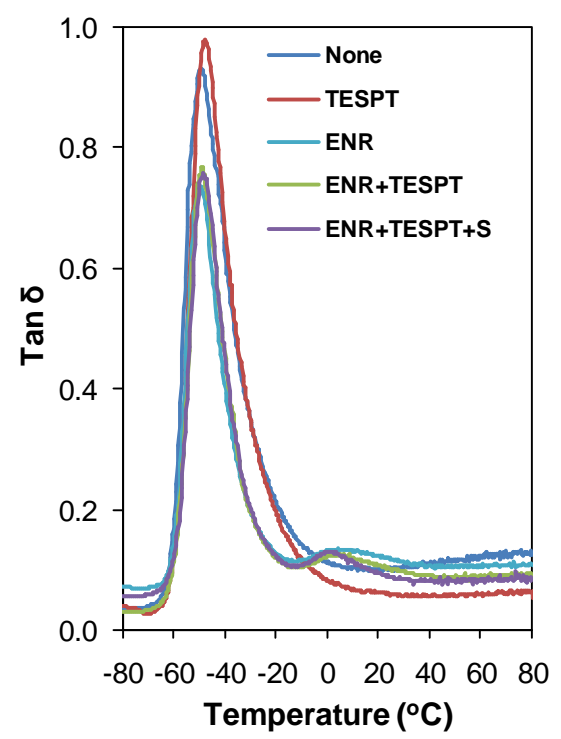

Figure 4.9 Tan $\delta$ of silica-filled NR vulcanizates containing different compatibilizer types and combinations.

Figures 4.8(a) and 4.8(b) clearly show that the addition of the different types of compatibilizers into the silica-filled NR vulcanizates has little effect on the storage ( $\left.E^{\prime}\right)$ and loss ( $E ")$ moduli in the glassy state and in the glass-to-rubber softening region, but significantly affects the material properties in the rubbery region. The different extent of interactions between the various components, the presence of ENR blended with NR and filler dispersion all play a role in the ability of the rubber chains to move and respond to deformation. After passing through the glass transition, the vulcanizates that contain ENR51 as compatibilizer, either without or with TESPT, show higher storage and loss moduli than the ones with TESPT and without any compatibilizer. The presence of 7.5 phr ENR51 with high epoxide-content and a glass transition temperature of $\pm 0^{\circ} \mathrm{C}$ causes a certain chain stiffness. Therefore, the NR with ENR-51 as compatibilizer has a higher elastic modulus, and in the mean time also shows a higher viscous or loss moludus because of less chain flexibility, as seen in Figure 4.8.

The use of the optimal content of $4.7 \mathrm{phr}$ TESPT gives vulcanizates with the highest tan $\delta$ peak due to improvement of silica dispersion, leading to less trapped rubber in the silica aggregates and so more rubber segments to respond to the dynamic 
deformation in this region. On the other hand, the use of ENR as compatibilizer results in a decrease of the tan $\delta$ peak intensity, even in combination with TESPT. This can partially be caused by the presence of the ENR which at this temperature is still in the glassy state, and may also result from interactions between the ENR and silica and self-crosslinked ENR that restrict motions of the rubber segments. In the rubbery region, the silica/TESPT system provides the lowest tan $\delta$ value due to the good chemical bonding between silica and rubber via silane bridges which help to reduce energy loss under dynamic conditions. The higher network density, as indicated by the rheometer maximum torque in Figures 4.4(a) and 4.4(b), enhances the elasticity further, resulting in lower loss tangent as well. The presence of ENR-51 that possesses higher damping properties results in the higher loss tangent in the temperature range of 0 to $80^{\circ} \mathrm{C}$ above its glass transition temperature of around $0^{\circ} \mathrm{C}$. The compound without any compatibilizer also shows an increasing trend of the loss tangent with rise of the test temperature.

The glass transition temperatures $\left(T_{g}\right)$ is the viscoelastic glass-to-rubber softening transition which is an important property of amorphous and semi-crystalline polymers. By dynamic mechanical analysis, the $T_{g}$ of polymers can be determined by using the peak temperatures of either $\tan \delta$ or loss modulus $\left(E^{\prime \prime}\right)$. Which point is to be used to at best represent $\mathrm{T}_{\mathrm{g}}$ is still a subject of debate. Robertson et al. ${ }^{[27]}$ discussed that $\tan \delta$ in the glass-to-rubber softening transition is determined not only by the local segmental motions of the polymer, as reflected in the loss modulus towards lower temperature, but also by the filler reinforcement effect on both the storage and loss moduli at higher temperature. They reported that the loss modulus peak which corresponds to the segmental relaxation process is not significantly affected by the particle surface area of the filler and degree of filler-polymer interactions ${ }^{[27]}$, whereas the shape and magnitude of the $\tan \delta$ peak are influenced by these parameters associated with the nature of the filler.

The present work reports the $T_{g}$ 's of the silica-filled NR vulcanizates containing none and different contents of TESPT, in combination with 7.5 phr of ENR-51, with and without sulfur compensation, as determined by using the peaks of both tan $\delta$ and loss modulus, as shown in Figure 4.10. The compounds with different degrees of filler-rubber interactions, as indicated by their bound rubber contents in Figure 4.3, show the same shapes and magnitudes of $E^{\prime \prime}$ peak: Figure 4.8(b), but different shapes and magnitudes of the $\tan \delta$ vs. temperature peaks: Figure 4.10, in accordance with the work of Robertson et al. ${ }^{[27]}$ The $\mathrm{T}_{\mathrm{g}}$ 's of the silica-filled NR vulcanizates from both $\mathrm{E}^{\prime \prime}$ and (tan $\left.\delta\right)_{\max }$ show the same trend, merely shifted over a few degrees, where the $T_{g}$ of the vulcanizate with optimal content of $4.7 \mathrm{phr}$ TESPT which has the highest chemical bound rubber content 
as well (Figure 4.3), is the highest. The vulcanizates with extra sulfur that have a higher crosslink density compared to the ones with normal sulfur content show a slightly higher $\mathrm{T}_{\mathrm{g}}$ and increasing TESPT content in the ENR-compatibilized NR shows an insignificant effect on $T_{g}$.

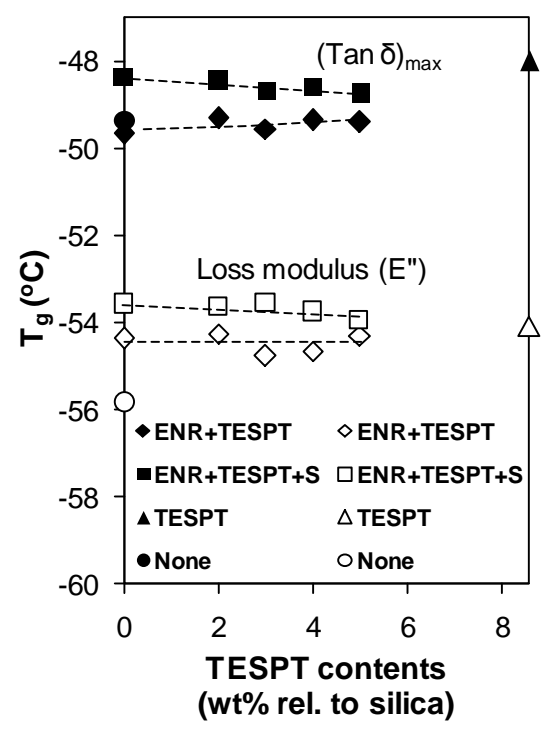

Figure 4.10 Glass transition temperatures $\left(T_{g}\right)$ at the peaks of $\tan \delta$ (solid symbols) and loss moduli (open symbols) of silica-filled NR vulcanizates with different compatibilizer combinations.

A study on the viscoelastic properties of crosslinked NR by DMA ${ }^{[28]}$ showed that the glass transition temperature depended somewhate on both the crosslink density and the crosslink types. A higher crosslink density increased the $T_{g}$ because of a more restrictions on molecular chain motions, as is commonly observed. Furthermore, the use of higher sulfur contents lead to a higher proportion of polysulfidic crosslinks and cyclic sulfidic structures which had a greater influence on $\mathrm{T}_{\mathrm{g}}$ than monosulfidic or carbon-carbon crosslink types. ${ }^{[28]}$ The chemically bonded rubber to the silica surface via silane bridges further restricts the movement of polymer chains and increases the $T_{g}$. This is in accordance with the work that reported the shift of $T_{g}$ towards higher temperature by polymer-filler coupling bonds. ${ }^{[5]}$ 

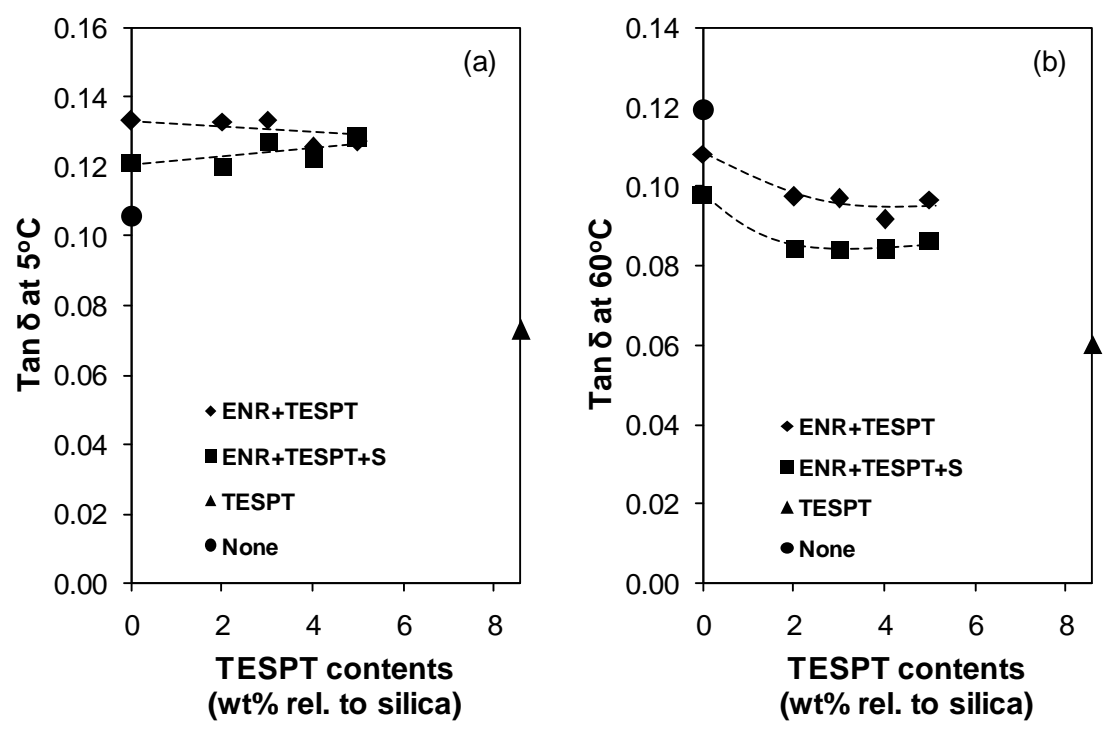

Figure 4.11 Tan $\delta$ at $5^{\circ} \mathrm{C}(\mathrm{a})$ and $60^{\circ} \mathrm{C}$ (b) of silica-filled NR vulcanizates without and with different compatibilizer combinations.

In the test method for wet grip grading of $\mathrm{C} 1$ tires according to UNECE Regulation $\mathrm{R} 117^{[29]}$, the wet surface temperature range for normal or summer tires is 5$35^{\circ} \mathrm{C}$, and for winter tires $2-20^{\circ} \mathrm{C}$. Figures $4.11(\mathrm{a})$ and $(\mathrm{b})$ show tan $\delta$ values at $5^{\circ} \mathrm{C}$ and $60^{\circ} \mathrm{C}$ for the silica-filled NR-vulcanizates with different compatibilizers. Higher tan $\delta$ at low temperature indicates a better tire wet grip. As shown in Figure $4.11(\mathrm{a})$, the tan $\delta$ at $5^{\circ} \mathrm{C}$ of the silica-filled NR vulcanizates is substantially increased with ENR added as compatibilizer, due to the damping behavior of epoxidized rubber influenced by its $T_{g}$ as exhibited in Figure 4.10. The use of TESPT in the range of $2-5 w t \%$ relative to the silica content in combination with ENR has only little effect on tan $\delta$. The silica-filled NR vulcanizate with TESPT shows the lowest tan $\delta$ at $5^{\circ} \mathrm{C}$ implying the lowest tire wet skid resistance. The chemical bonds between silica and rubber via silane molecules give an additional contribution to the crosslink network that is normally generated by the vulcanization reaction. This results in a better elastic response and lower energy loss. This double network also causes the lowest tan $\delta$ at $60^{\circ} \mathrm{C}$ which indicates a low rolling resistance for tires, as shown in Figure 4.11(b). The presence of ENR-51 leads to a significantly higher tan $\delta$ at $60^{\circ} \mathrm{C}$ compared to the use of TESPT alone, but is still lower than for the non-compatibilized compound. The addition of TESPT of just only $2 \mathrm{wt} \%$ relative to silica shows an improvement of $\tan \delta$ at $60^{\circ} \mathrm{C}$ due to the improved filler dispersion and cure behavior. The correction of sulfur-contents in the ENR-compatibilized 
compounds decreases tan $\delta$ at both $0^{\circ} \mathrm{C}$ and $60^{\circ} \mathrm{C}$ of the vulcanizates due to the increase of crosslink density, leading to better rubber elasticity.

4.3.6 SEM images of silica-filled NR with ENR, TESPT and extra sulfur as compatibilizers, with and without sulfur compensation

Filler dispersion level is one of the parameters that affect the properties of filled compounds and vulcanizates. The silica dispersion in the NR matrix containing different types of compatibilizers was studied by the SEM technique using two different sets of specimens, as described in Section 4.2.3. For the cryogenically cracked surfaces as shown in Figure 4.12, the compounds contain only $30 \mathrm{phr}$ of silica in order to be able to better observe the silica dispersion. For the regular formulation with $55 \mathrm{phr}$ of silica, tensile fractured surfaces were used and the SEM micrographs are shown in Figure 4.13.
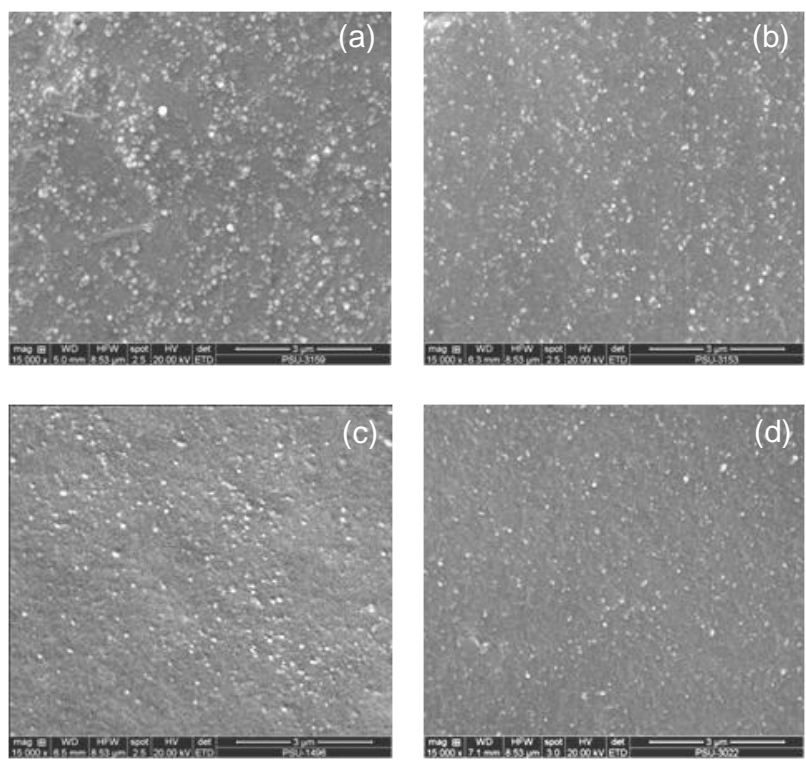

Figure 4.12 SEM micrographs of filled NR vulcanizates with $30 \mathrm{phr}$ of silica at $15000 \mathrm{x}$ magnification: (a) without compatibilizer; (b) with 7.5 phr ENR-51; (c) with 7.5 phr ENR-51 plus $4 \mathrm{wt} \%$ TESPT rel. to silica; and (d) with $8.6 \mathrm{wt} \%$ TESPT rel. to silica.

Due to a large number of silanol groups on the silica surface, breaking of silica agglomerates and dispersion of aggregates into the rubber matrix are difficult as a result of hydrogen bonding. The silica dispersion of the filled NR vulcanizate without compatibilizer: Figure 4.12(a), is clearly poorer than the vulcanizates that contain 
compatibilizers, as the silica is less homogeneously dispersed in the matrix and aggregates/agglomerate size is larger than what can be observed in the other compounds. The incorporation of ENR-51 into the silica-filled NR improves the silica dispersion in the matrix as visually observed in Figure 4.12(b) due to interactions between the silanol groups of the silica and epoxide groups of ENR leading to hydrophobation of the silica. The dispersion of silica is further improved by the use of ENR in combination with TESPT at $4 \mathrm{wt} \%$ relative to the silica, as seen in Figure 4.12(c), in which the silica is finely dispersed to more or less the same level as that of the silica-filled NR vulcanizate with optimal content of $4.7 \mathrm{phr}$ TESPT: Figure 4.12(d). The SEM results are in accordance with the Payne effect: Figure 4.2(a), and the resulting vulcanizate properties in which the non-compatibilized system is the worst. The addition of TESPT to react with free silanol groups through the silanization reaction to form siloxane bonds and the bound silane on the silica surface suppress the filler-filler interactions and prevents the silica from re-agglomeration. The better silica dispersion in NR with TESPT silane coupling agent compared with vulcanizates without silane was also clearly demonstrated by SEM images of cut-off surfaces by Choi et al. ${ }^{[30]}$
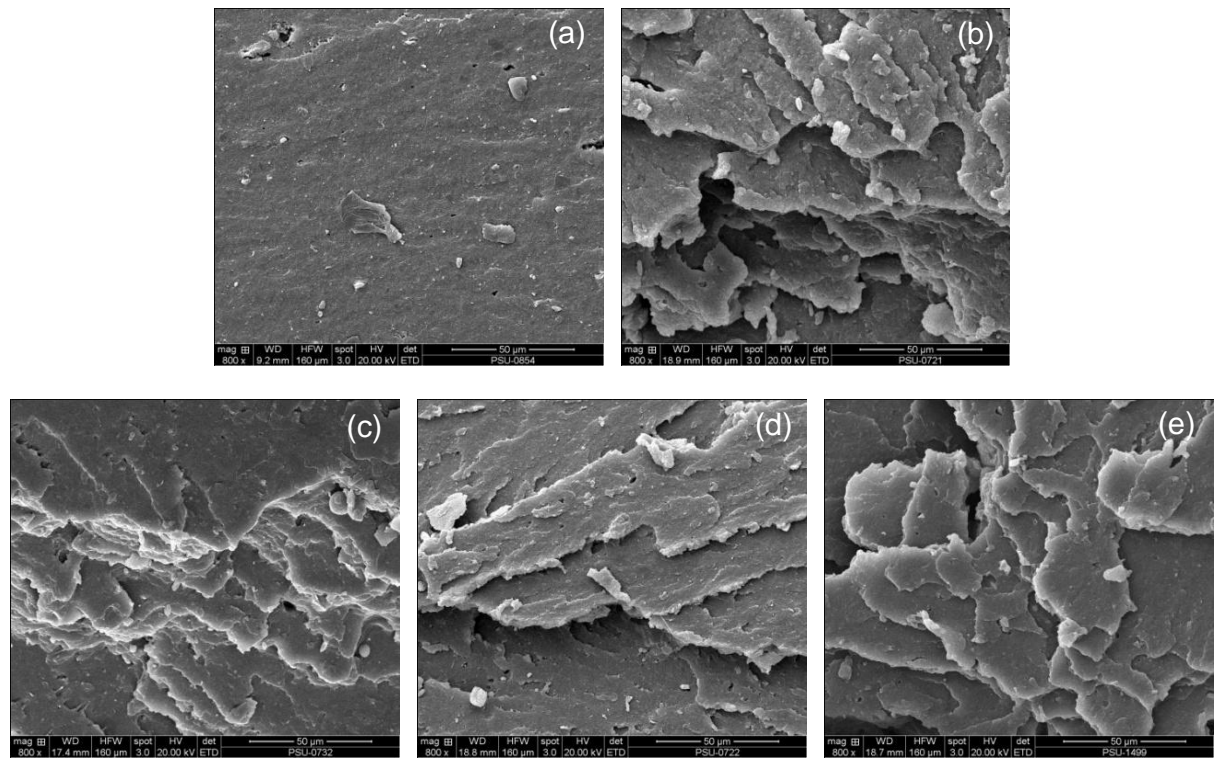

Figure 4.13 SEM micrographs of tensile fractured surfaces of filled NR vulcanizates with 55 phr of silica at $800 x$ magnification: (a) without compatibilizer; (b) with 8.6 wt\% TESPT rel. to silica; (c) with 7.5 phr ENR-51; (d) with 7.5 phr ENR-51 plus 4 wt\% TESPT rel. to silica; and (e) with 7.5 phr ENR-51, 4 wt\% TESPT rel. to silica and extra sulfur. 
SEM micrographs of tensile fractured surfaces of the silica-filled NR vulcanizates containing different compatibilizers are shown in Figure 4.13. The silica-filled NR vulcanizate without compatibilizer that has the lowest tensile strength because of the poor filler dispersion and filler-rubber interactions, shows a smooth surface with some vacuoles on the failure surface indicating de-wetting of silica by the NR matrix, as shown in Figure 4.13(a). The observation of silica agglomerates by SEM in silica-filled NR without compatibilizer was previously demonstrated by $\mathrm{Pal}$ and De. ${ }^{[31]}$ The effective use of TESPT silane coupling agent in the silica-filled NR that leads to the highest modulus: Figure 4.6(a), and tensile strength: Figure 4.7(a), gives a rough fractured surface with many tear lines from ductile failure ${ }^{[32]}$ as a result of good interfacial interactions between rubber and silica by silane coupling. All of the fractured surfaces of silica-filled NR with ENR-51, ENR51 in combination with TESPT and ENR-51 in combination with TESPT and extra sulfur as compatibilizers as shown in Figure 4.13(c-e) respectively, show a rough surface and tear lines pattern but at different degrees compared with the silica/TESPT system. The ENRcompatibilized vulcanizate with improved filler-rubber interactions via the interaction/reaction between the epoxide groups of ENR and the silanol groups on the silica surface, has a considerably less complicated fractured surface when compared to the one with optimal content of TESPT. The silica-filled vulcanizates with TESPT and ENR/TESPT/extra sulfur show the same level of tensile strength as shown in Figure 4.7(a) show very similar tensile fractured surfaces, as shown in Figure 4.13(c) and 4.13(e), respectively. SEM micrographs of tensile fractured surfaces of filled NR vulcanizates have also been used to demonstrate dispersion and wetting of filler by the matrix, such as in the work of Arayapranee et al. ${ }^{[32]}$ and Ismail et $a l^{[33]}$ in which the rough surfaces with tear lines indicated higher tensile strength.

\subsection{Conclusions}

The use of ENR-51 at 7.5 phr in combination with TESPT silane coupling agent at $2 \mathrm{wt} \%$ relative to silica further decreases Mooney viscosity, Payne effect, flocculation rate constant and increases bound rubber contents of silica-filled NR compounds, when compared to a silica-filled compound with ENR-51 alone. A further increase of TESPTcontent has only a small additional effect on the compound properties. Scorch and cure times of the compounds decrease by increasing TESPT-contents and the addition of extra sulfur decreases the scorch time further. Tensile strength and modulus of the vulcanizates increase with increasing TESPT-contents and further improve by sulfur compensation relative to the reference compound with TESPT. The use of ENR-51 at $7.5 \mathrm{phr}$ with 
TESPT at 4 wt\% relative to silica and extra sulfur gives vulcanizates with the same levels of tensile strength compared to the vulcanizates with TESPT at $8.6 \mathrm{wt} \%$ relative to silica. The dynamic mechanical properties of the silica-filled NR vulcanizates are significantly affected by the presence of ENR-51 that has a higher damping than NR. Above the glassto-rubber region, the ENR-containing vulcanizates show higher storage and loss modulus and higher $\tan \delta$ compared to the silica/TESPT system. The addition of TESPT to the ENR-compatibilized compound cause only a small change in $\mathrm{T}_{\mathrm{g}}$ and $\tan \delta$ at $5^{\circ} \mathrm{C}$, but lower $\tan \delta$ at $60^{\circ} \mathrm{C}$. The addition of extra sulfur leads to an increase of $\mathrm{T}_{\mathrm{g}}$ and lower $\tan \delta$ at both $5^{\circ} \mathrm{C}$ and $60^{\circ} \mathrm{C}$ as a result of increased crosslink density. The SEM micrographs clearly show good silica dispersion in all compatiblized vulcanizates, and the tensile fracture surfaces of the materials show different tear line patterns dependent on their tensile strengths.

Overall, the combination of ENR-51, TESPT and sulfur compensation does not fully match the properties of a compound with TESPT alone at optimal quantity. However, if in the perspective of the "Magic Triangle of Tire Technology" the wet skid resistance needs to be boosted versus rolling resistance, e.g. for "Winter Tires", then the combination of ENR-51, TESPT and sulfur compensation presents itself as a better option: which has to be confirmed in real tire tests.

\subsection{References}

1. R. Rauline, EP Patent 0501227A1, to Michelin \& Cie, February 9, 1992.

2. S. Wolff, Kautsch. Gummi Kunstst., 41, 674 (1988).

3. N. Suzuki, M. Ito, S. Ono, J. Appl. Polym. Sci., 95, 74 (2005).

4. P. Sae-oui, C. Sirisinha, U. Thepsuwan, K. Hatthapanit, Eur. Polym. J., 43, 185 (2007).

5. A.K. Manna, P.P. De, D.K. Tripathy, J. Appl. Polym. Sci., 84, 2171(2002).

6. W. Kaewsakul, K. Sahakaro, W.K. Dierkes, J.W.M. Noordermeer, Rubber Chem. Technol., 85, 277 (2012).

7. J.W. ten Brinke, S.C. Debnath, L.A.E.M. Reuvekamp, J.W.M. Noordermeer, Comp. Sci. Technol., 63, 1165 (2003).

8. A.S. Hasham, B. Azahari, Y. Ikeda, S. Kohjiya, Rubber Chem. Technol., 71, 289 (1998).

9. A.D. Roberts, "Natural Rubber Science and Technology", Oxford University Press, New York, 1988. 
10. M. Arroyo, M.A. López-Manchado, J.L. Valentín, J. Carretero, Comp. Sci. Technol., 67, 1330 (2007).

11. P.L. Teh, Z.A. Mohd Ishak, A.S. Hashim, J. Karger-Kocsis, U.S. Ishiaku, Eur. Polym. J., 40, 2513 (2004).

12. F. Cataldo, Macromol. Mater. Eng., 287, 348 (2002).

13. A.K. Manna, A.K. Bhattacharyya, P.P. De, D.K. Tripathy, S.K. De, D.G. Peiffer, Polymer, 39, 7113 (1998).

14. W. Kaewsakul, K. Sahakaro, W.K. Dierkes, J.W.M. Noordermeer, Rubber Chem. Technol., 87, 291 (2014).

15. P. Saramolee, K. Sahakaro, N. Lopattananon, W.K. Dierkes, J.W.M. Noordermeer, Adv. Mat. Res., 747, 522 (2013).

16. P. Saramolee, K. Sahakaro, N. Lopattananon, W.K. Dierkes, J.W.M. Noordermeer, Adv. Mat. Res., 844, 235 (2014).

17. H.-D. Luginslands, J. Fröhlich, A. Wehmeier, Rubber Chem. Technol., 75, 563 (2002).

18. J. Zhi-xin, X. Ti-wen, L. Yuan-fang, J. De-min, International Rubber Conference, Beijing, September 16-18, 2014.

19. M.-J. Wang, S. Woff, J.-B. Donnet, J.-B. Donnet, Rubber Chem. Technol., 64, 559 (1991).

20. S. Wolff, M.-J. Wang, E.-H. Tan, Rubber Chem. Technol., 66, 163 (1993).

21. S.-S. Choi, Polym. Test., 21, 201 (2002).

22. S. Wolff, Rubber Chem. Technol., 69, 325 (1996).

23. P.L. Teh, Z.A. Mohd Ishak, A.S. Hashim, J. Karger-Kocsis, U.S. Ishiaku, J. Appl. Polym. Sci., 94, 2438 (2004).

24. B.T. Poh, H. Ismail, E.H. Quah, P.L. Chin, J. Appl. Polym. Sci., 81, 47 (2001).

25. C.J. Lin, W.L. Hergenrother, E. Alexanian, G.G.A. Böhm, Rubber Chem. Technol., 75, 865 (2002).

26. L.J. Murphy, E. Khmelnitskaia, M.J. Wang, K. Mahmud, Rubber Chem. Technol., 71, 1015 (1998).

27. C.G. Robertson, C.J. Lin, M. Rackaitis, C.M. Roland, Macromolecules, 41, 2727 (2008).

28. R. Hagen, L. Salmén, B. Stenberg, J. Polym. Sci. Part B: Polym. Phys., 34, 1997 (1996).

29. UNECE Regulation No 117, GRRF $71^{\text {st }}$ session, September 13-15, 2011.

30. S.-S. Choi, C. Nah, B.-W. Jo, Polym. Int., 52, 1382 (2003).

31. P.K. Pal, S.K. De, Rubber Chem. Technol., 55, 1370 (1982). 
32. W. Arayapranee, N. Na-Ranong, G.L. Rempel, J. Appl. Polym. Sci., 98, 34 (2005).

33. H. Ismail, R. Nordin, A.M. Noor, Polym. Test., 21, 565 (2002). 


\title{
Silica-Reinforced Natural Rubber Compounds Compatibilized by ENR in combination with Different Silane Coupling Agent Types
}

\begin{abstract}
Bis-(triethoxysilylpropyl)tetrasulfide (TESPT), 3-mercaptopropyl-di(tridecan-1oxy-13-penta(ethyleneoxide))ethoxysilane (VP Si-363 ${ }^{T M}$ ) and 3-octanoyl-thiopropyltriethoxysilane $\left(N X T^{T M}\right)$ are comparatively studied at varying amounts from 2 to 5 $w t \%$ relative to the silica, in combination with $7.5 \mathrm{phr}$ of epoxidized natural rubber with 51 mol\% of epoxide (ENR-51), as compatibilizers for silica-filled NR compounds. The use of different types of silane coupling agents together with ENR gives silica-filled NR compounds with different Mooney viscosities, bound rubber contents, cure properties and tensile properties. Over the range of silane contents used with ENR, the compounds with VP Si-363 and NXT show a lower Mooney viscosity, bound rubber content and moduli at $100 \%$ and $300 \%$ elongation when compared to the one with TESPT. The ENR/NXT combinations give the best scorch safety. As the presence of ENR-51 at 7.5 phr already effectively reduces the filler-filler interactions, the addition of silane on top of the ENR has only little influence on Mooney viscosity and Payne effect, but clearly enhances fillerrubber interactions as indicated by an increased chemically bound rubber content. The tensile properties of the silica-filled NR vulcanizates are increased with increasing silane contents, in which state-of-the-art TESPT still provides better properties than the other silanes, while VP Si-363 and NXT give more or less the same properties. At a fixed silane content of 4 wt\% relative to silica and 7.5 phr of ENR-51, sulfur compensation added into the compounds by taking the mix with optimal content of TESPT as reference, enhances the tensile modulus and strength. The differences in tensile properties of the vulcanizates containing different compatibilizer combinations are also reflected in their different tear line patterns on tensile fractured surfaces, as analyzed by the SEM technique. In the presence of ENR, the dynamic mechanical properties of the silica-filled NR compounds either with or without silane are influenced by the glass transition temperature of the ENR. With respect to tire rolling resistance, the use of TESPT still provides the lowest loss tangent at $60^{\circ} \mathrm{C}$.
\end{abstract}




\subsection{Introduction}

Incorporation of fillers into rubber compounds has an effect on the physical properties of the rubber vulcanizates that results from the development of stress concentration, which in turn depends on several factors such as shape of the filler, the filler surface, particle size, particle size distribution, and nature of the boundary layer between filler and matrix. ${ }^{[1-3]}$ Silica is a non-black filler which consists of a great number of silanol groups on its surface, leading to high polarity, acidity and low adhesion between silica and elastomers. ${ }^{[2]}$ Use of silica as a reinforcing filler in rubber compounds needs some chemical functional groups or silane coupling agent to increase the reinforcing efficiency by means of increased silica-rubber interactions and improved silica dispersion. The use of epoxidized natural rubber (ENR) as compatibilizer in silica-filled NR compounds, as shown in Chapter 3, demonstrated that it could enhance the reinforcing efficiency of silica to some extent but the final properties were still somewhat inferior to the use of state-of-the-art TESPT silane coupling agent. It is known that the properties of ENR itself depend on the epoxide-group content and that there is a decrease of strain-induced crystallization at high levels of epoxidation. ${ }^{[4,5]}$ Various studies on silica-filled ENR compounds $^{[6-8]}$ reported good reinforcement and improved properties compared to silicafilled NR without silane coupling agent, due to chemical interactions between the polar epoxide-groups of the ENR and the silanol-groups on the silica surface. ${ }^{[7,8]}$

The most widely used method to optimize the silica reinforcing efficiency in rubber compounds is the use of a silane coupling agent. The silane modified silica surface has a reduced specific surface energy, leading to improvement of the compatibility between silica and hydrocarbon rubber, better silica dispersion, improved compound processability and vulcanization properties. ${ }^{[9]}$ Sulfur-containing silane coupling agents have more influence on the overall properties of silica-filled rubber than a sulfur-free silane. ${ }^{[10]}$ Bis-(triethoxysilylpropyl)tetrasulfide (TESPT) gives better scorch safety and lower compound viscosity when compared to $\mathrm{y}$-mercaptopropyltriehoxysilane (MPS) due to the reactive thiol end-group of the MPS which has a catalytic effect on accelerated sulfur vulcanization, whereas TESPT has steric hindrance by bulky ethoxy end-groups. ${ }^{[11]}$ TESPT with its tetrasulfide moiety is known to donate free sulfur to the compounds, and the use of a silane with lower sulfur rank or a sulfur-free silane needs correction of the sulfur-content relative to the sulfur contained in the reference compound with TESPT. It has been reported that the extra sulfur added either in the first step of mixing in an internal mixer or in the second step on a two-roll mill can react with the coupling agent to some extent, leaving a large part in the system to participate in the vulcanization reaction. ${ }^{[10]}$ 
Now silica is constantly gaining importance for energy-saving tires, there is also a steady development of alternative silanes. 3-Octanoyl-thio-propyltriethoxysilane (NXT) has been introduced as a silane coupling agent offering a better scorch safety than TESPT, because of its bulky blocking end-group on the mercapto function. ${ }^{[12-14]}$ The blocked silane will couple with NR only during the curing period ${ }^{[13,14]}$ and it has been reported that the NXT-silane needs a mixing temperature above $130^{\circ} \mathrm{C}$ to react with the silanol groups on silica. The longer scorch time of NXT compound is in accordance with the higher activation energy for premature scorch compared to that of TESPT. ${ }^{[13]}$ A study on SBR compounds filled with silica that were modified by NXT in comparison with TESPT showed that the NXT compounds showed lower Payne effects and lower viscosities due to better filler dispersion, but poorer modulus because the silane contains only one sulfur atom in its structure. ${ }^{[15]}$ Another new silane that was proposed for tire compounds is VP Si-363 which is a bifunctional organosilane having long dialkoxy-ethoxy-silyl groups and a mercapto function. ${ }^{[16]}$ The "scorchy" nature of the mercapto function of this silane is suppressed by steric hindrance by the large side-arms. ${ }^{[16]}$ As the silica-silane reaction proceeds via the ethoxy-group, this silane type produces less ethanol emission during processing compared to the conventional TESPT or Si-69. A kinetic study by Blume ${ }^{[17]}$ using a model system showed that the presence of $-\mathrm{SH}$ and long alkoxy groups enhances the rate of reaction. Furthermore, the oxygen atoms inside the long alkoxy chain of VP Si363 may increase the possibility of silane adsorption onto the silanol groups of the silica via hydrogen bonding and shield the free silanol groups.

Based on the results in Chapter 3, that dealt with the effect of epoxidized natural rubbers (ENRs) as compatibilizers in silica-filled NR, the use of ENR containing $51 \mathrm{~mol} \%$ of epoxide (ENR-51) at 7.5 phr gave the best overall improvement in the Payne effect, bound rubber content, mechanical and dynamic mechanical properties. With the aim to reduce the use of silane coupling agent and to reduce ethanol emission, this chapter explores combinations of ENR-51 at $7.5 \mathrm{phr}$ with three different types of silane: TESPT, VP Si-363 and NXT in silica-filled NR-compounds. The amount of ENR-51 is fixed at 7.5 phr based on the optimum properties as reported in Chapter 3 , and the silane contents are varied from 2.0 to $5.0 \mathrm{wt} \%$ relative to the silica amounts. Later on, with fixed amounts of both ENR-51 and silane, compensations are applied in the compound with elemental sulfur relative to the total sulfur contained in the reference TESPT-compound, and the resulting properties are investigated in comparison with the normal formulation. 


\subsection{Experimental}

\subsubsection{Materials}

All of the compounding ingredients used in this chapter are the same as described in Chapter 3. Three different types of silane coupling agents were used including bis-(triethoxysilylpropyl)tetrasulfide (TESPT) (Evonik, Germany), 3mercaptopropyl-di(tridecan-1-oxy-13-penta(ethyleneoxide))ethoxysilane (VP Si-363 ${ }^{\mathrm{TM}}$ ) (Evonik, Germany) and 3-octanoyl-thio-propyltriethoxysilane (NXT'T) (Momentive, USA). Their details are given in Table 5.1 .

Table 5.1 Silane coupling agent information.

\begin{tabular}{|c|c|c|c|}
\hline $\begin{array}{l}\text { Silane } \\
\text { types }\end{array}$ & Chemical names & Structures & $\begin{array}{c}\text { MW } \\
(\mathrm{g} / \mathrm{mol})\end{array}$ \\
\hline TESPT & $\begin{array}{l}\text { Bis-(triethoxysilylpropyl) } \\
\text { tetrasulfide }\end{array}$ & 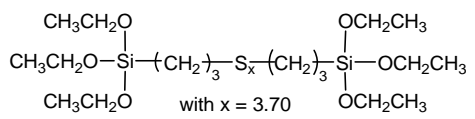 & 532.5 \\
\hline $\begin{array}{l}\text { VP Si- } \\
363\end{array}$ & $\begin{array}{l}\text { 3-Mercaptopropyl-di(tridecan- } \\
\text { 1-oxy-13-penta } \\
\text { (ethyleneoxide))ethoxysilane }\end{array}$ & $\begin{array}{c}\mathrm{CH}_{3}\left(\mathrm{CH}_{2}\right)_{12}\left(\mathrm{OC}_{2} \mathrm{H}_{4}\right)_{5} \mathrm{O} \\
\left.\mathrm{CH}_{3} \mathrm{CH}_{2} \mathrm{O}-\mathrm{Si}-\mathrm{CH}_{2}\right)_{3}-\mathrm{SH} \\
\mathrm{CH}_{3}\left(\mathrm{CH}_{2}\right)_{12}\left(\mathrm{OC}_{2} \mathrm{H}_{4}\right)_{5} \mathrm{O}\end{array}$ & 987.5 \\
\hline NXT & $\begin{array}{l}\text { 3-Octanoyl-thio- } \\
\text { propyltriethoxysilane }\end{array}$ & $\begin{array}{c}\mathrm{CH}_{3} \mathrm{CH}_{2} \mathrm{O} \\
\underset{\mathrm{CH}_{3} \mathrm{CH}_{2} \mathrm{O}-\mathrm{Si}}{\mathrm{CH}_{3} \mathrm{CH}_{2} \mathrm{O}}\end{array}$ & 365 \\
\hline
\end{tabular}

\subsubsection{Compound formulations and mixing procedures}

The first mixing step of silica-filled NR compounds in this chapter was performed using an internal mixer, Brabender ${ }^{\circledR}$ 50EHT, (Brabender ${ }^{\circledR} \mathrm{GmbH}$ \& Co.KG, Germany) with a mixing chamber volume of $70 \mathrm{~cm}^{3}$. The initial mixer temperature setting was $100^{\circ} \mathrm{C}$ in order to reach a dump temperature in the range of $135-150^{\circ} \mathrm{C}$, as reported by Kaewsakul et al. ${ }^{[15]}$, that is the temperature range providing optimal overall properties of the silica/silane-filled NR compounds. The compound formulations are shown in Tables 5.2 and 5.3, and the mixing procedures are described in Table 5.4. The second step of curative addition was carried out on a two-roll mill. 
Table 5.2 Compound formulations without extra sulfur.

\begin{tabular}{lccc|ccc}
\hline \multirow{2}{*}{ Ingredients } & \multicolumn{6}{c}{ Parts per hundred parts of rubber (phr) } \\
\cline { 2 - 7 } & \multicolumn{5}{c}{ References } & \multicolumn{3}{c}{ No extra sulfur } \\
\hline RSS3 & 100.0 & 100. & 92.5 & 92.5 & 92.5 & 92.5 \\
& & 0 & & & & \\
ENR-51 & - & - & 7.5 & 7.5 & 7.5 & 7.5 \\
TESPT & - & $4.7^{\star}$ & - & $1.1-2.7^{* *}$ & - & - \\
VP Si-363 & - & - & - & - & $1.1-2.7^{* *}$ & - \\
NXT & - & - & - & - & - & $1.1-2.7^{* *}$ \\
Zeosil & 55.0 & 55.0 & 55.0 & 55.0 & 55.0 & 55.0 \\
1165MP & & & & & & \\
TDAE oil & 8.0 & 8.0 & 8.0 & 8.0 & 8.0 & 8.0 \\
ZnO & 3.0 & 3.0 & 3.0 & 3.0 & 3.0 & 3.0 \\
TMQ & 1.0 & 1.0 & 1.0 & 1.0 & 1.0 & 1.0 \\
Stearic acid & 1.0 & 1.0 & 1.0 & 1.0 & 1.0 & 1.0 \\
DPG & 1.0 & 1.0 & 1.0 & 1.0 & 1.0 & 1.0 \\
CBS & 1.5 & 1.5 & 1.5 & 1.5 & 1.5 & 1.5 \\
Sulfur & 1.5 & 1.5 & 1.5 & 1.5 & 1.5 & 1.5 \\
\hline
\end{tabular}

Remarks: *TESPT 4.7 phr equals to $8.6 \mathrm{wt} \%$ rel. to the silica; ${ }^{* *}$ Amounts of silane were varied at 2,3 , $4,5 \mathrm{wt} \%$ rel. to silica, which are respectively equal to $1.1,1.65,2.2,2.7 \mathrm{phr}$;

Table 5.3 Compound formulations with sulfur compensation.

\begin{tabular}{lcccc}
\hline \multirow{2}{*}{ Ingredients } & \multicolumn{4}{c}{ Parts per hundred parts of rubber } \\
& \multicolumn{4}{c}{ Exhr) } \\
\cline { 2 - 5 } & 92.5 & 92.5 & 92.5 & 92.5 \\
\hline RSS3 & 7.5 & 7.5 & 7.5 & 7.5 \\
ENR-51 & - & 2.2 & - & - \\
TESPT & - & - & 2.2 & - \\
VP Si-363 & - & - & - & 2.2 \\
NXT & 55.0 & 55.0 & 55.0 & 55.0 \\
Zeosil 1165MP & 8.0 & 8.0 & 8.0 & 8.0 \\
TDAE oil & 3.0 & 3.0 & 3.0 & 3.0 \\
ZnO & 1.0 & 1.0 & 1.0 & 1.0 \\
TMQ & 1.0 & 1.0 & 1.0 & 1.0 \\
Stearic acid & 1.0 & 1.0 & 1.0 & 1.0 \\
DPG & 1.5 & 1.5 & 1.5 & 1.5 \\
CBS & 2.6 & 2.1 & 2.5 & 2.4 \\
Sulfur &
\end{tabular}

Remarks: *sulfur content was compensated relative to the sulfur contained in the reference compound with $4.7 \mathrm{phr}$ TESPT. 
Table 5.4 Mixing procedures.

\begin{tabular}{lc}
\multicolumn{1}{c}{ Mixing procedures } & $\begin{array}{c}\text { Cumulative } \\
\text { time (mins.) }\end{array}$ \\
\hline Step $1:$ Internal mixer & 2 \\
- NR and ENR mastication & 7 \\
- Addition of a first half of silica (and $1 / 2$ of silane) & 12 \\
- Addition of a second half of silica( and $1 / 2$ silane) and TDAE oil & 15 \\
- Addition of ZnO, stearic acid, and TMQ & 5 \\
\hline Step 2: Two roll mill & 5 \\
\hline
\end{tabular}

\subsubsection{Mooney viscosity, Payne effect and bound rubber content}

Mooney viscosity $\left[\mathrm{ML}(1+4), 100^{\circ} \mathrm{C}\right]$ was tested by using a Visc $\mathrm{Tech}^{+}$(Tech-Pro Inc., USA) according to ASTM D1646. The Payne effect or filler-filler interaction of the final compounds (with curatives) was analyzed by using a Rubber Process Analyzer (RPA2000, Alpha Technologies, USA) at $100^{\circ} \mathrm{C}$, frequency $0.5 \mathrm{~Hz}$ and varying strains from $0.56 \%$ to $100 \%$. Bound rubber content of the silica-filled NR non-productive compounds, i.e. the compounds without curatives, was analyzed following the procedures as described in Chapter 3.

\subsubsection{Cure characteristics, vulcanization and testing of tensile properties}

Cure characteristics of the silica-filled NR compounds were analyzed by using a Moving Die Rheometer (MDR) (rheoTech $\mathrm{MD}^{+}$, Tech-Pro, Inc., USA) at $150^{\circ} \mathrm{C}$ for 30 minutes at a frequency of $1.67 \mathrm{~Hz}$ and $13.95 \%$ strain. The compounds were later cured to their respective cure times $\left(\mathrm{t}_{\mathrm{c} 90}\right)$ with a compression mold (Chaicharoen Karnchang Ltd., Thailand). The vulcanized sheets of $2 \mathrm{~mm}$ thickness were cut into dumbbell specimens using die type $\mathrm{C}$, and tensile testing was carried out using a Hounsfield Tensile Tester (H10KS, Hounsfield Test Equipment, England) at a crosshead speed of $500 \mathrm{~mm} / \mathrm{min}$ according to ASTM D412.

\subsubsection{Dynamic mechanical properties}

Storage modulus, loss modulus and $\tan \delta$ of the silica-filled NR vulcanizates containing different compatibilizers were determined using a dynamic mechanical thermal analyzer, DMTA V (Rheometrics Scientific, USA). The samples were tested in the tension mode in a temperature range from $-80^{\circ} \mathrm{C}$ to $80^{\circ} \mathrm{C}$, at a frequency of $10 \mathrm{~Hz}$ under two strain deformations: $0.001 \%$ strain at $-80^{\circ} \mathrm{C}$ to $-30^{\circ} \mathrm{C}$ and $0.01 \%$ strain from $-30^{\circ} \mathrm{C}$ to $80^{\circ} \mathrm{C}$. 


\subsubsection{Scanning electron microscopy}

Tensile fractured surfaces of the silica-filled NR vulcanizates were gold sputter coated and characterized by Scanning Electron Microscopy (SEM) as described in Chapter 4.

\subsection{Results and discussion}

\subsubsection{Effect of silane coupling agents on the properties of ENR-compatibilized} silica-filled NR

\subsubsection{Mooney viscosity and Payne effect}
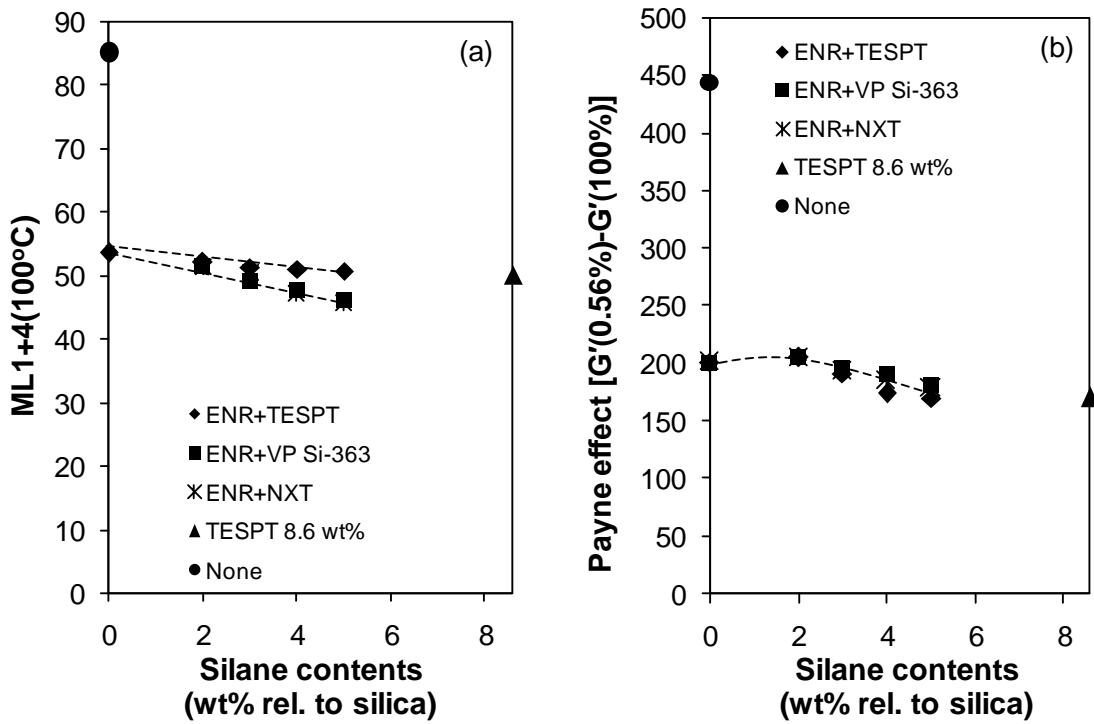

Figure 5.1 Mooney viscosity (a) and Payne effect (b) of silica-filled NR compounds with 7.5 phr of ENR-51 in combination with varying amounts of TESPT, VP Si-363 and NXT.

Mooney viscosities of the silica-filled NR compounds with different types and amounts of silane coupling agents added on top of 7.5 phr of ENR-51 as compatibilizer are shown in Figure 5.1(a). The silica-filled compound without compatibilizer shows by far the highest compound viscosity as previously observed in Figure 4.1, due to hindrance of rubber flow by the filler network and silica agglomerates. The presence of ENR-51 as compatibilizer significantly reduces the Mooney viscosity as a result of interactions between the epoxy groups of ENR and silanol groups on the silica surface, as discussed 
in Chapter 3 and proposed in Scheme 3.2. The addition of increasing amounts of silane further decreases the Mooney viscosity slightly, indicating an improvement of silica dispersion, as a small amount of free silanol groups may still exist in the system after the silica-ENR interactions. An excess amount of silane coupling agents could also act as plasticizer for the rubber compounds and reduce the compound viscosity.

The compounds with VP Si-363 and NXT silane coupling agents show lower Mooney viscosities than those with TESPT. The long alkoxy groups with polyglycol units of VP Si-363 enhance the hydrophobation and so improve silica dispersion as well as promote the rubber chain mobility around the silica surface ${ }^{[16]}$, whereas the blocking group in the NXT molecules can act as a plasticizing fragment that may result in a reduction of compound viscosity. Furthermore, the bulky side-groups of VP Si-363 and the blocking group of NXT prevent a reaction between the mercapto group and rubber polymer during processing. This is different from TESPT that has polysulfidic linkages which can disproportionate and donate sulfur atoms into the system to generate premature crosslinking during processing and may cause a higher compound viscosity. ${ }^{[10,18]}$ The Payne effect of the silica-filled NR compounds with different compatibilizers is shown in Figure 5.1(b). All the compatibilized compounds show similar Payne effects close to that of the reference TESPT compound, indicating that the filler-filler interactions are efficiently replaced by interactions between the epoxy groups of ENR and alkoxy groups of the silanes with the silanol groups on the silica surface. The increase of silane coupling agents to 4-5 wt\% relative to silica results in the same level of Payne effect as the compound with optimal TESPT dosage, and all the three types of silanes show no different effect on this property.

\subsubsection{Filler-rubber interaction}

Silica is an inorganic filler with a large number of polar silanol groups on its surface leading to a poor interaction with hydrocarbon rubbers. To improve the interfacial interaction between silica and rubber, silane coupling agents or other types of compatibilizers are generally added into the silica-filled rubber compounds, so that an enhancement of mechanical and dynamic mechanical properties can be achieved. Bound rubber content is a general parameter used to determine the degree of filler-rubber interaction in the compounds. The effects of silane types and concentrations on the bound rubber content of the ENR-compatibilized silica-filled NR compounds are shown in Figure 5.2. Therein, Figure 5.2 (a) shows the chemically bound rubber contents after the 
treatment with ammonia for cleaving physical interactions, and Figure 5.2 (b) displays the results of physically bound rubber content.
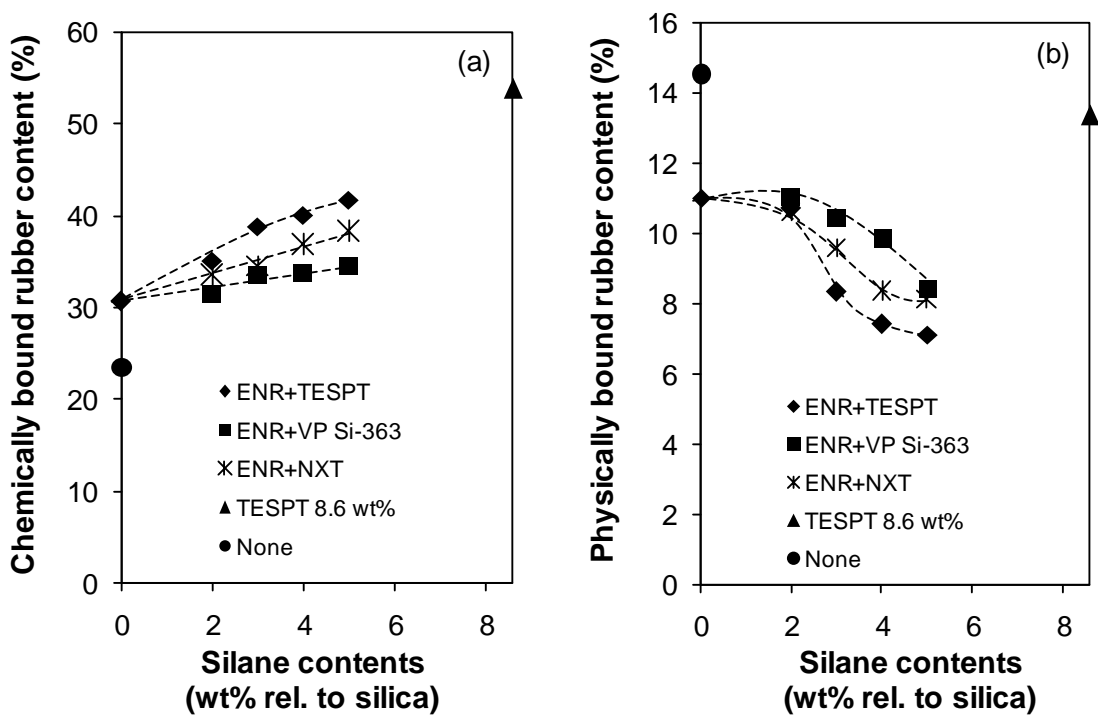

Figure 5.2 Chemically (a) and physically (b) bound rubber contents of silica-filled NR compounds with 7.5 phr of ENR-51 as compatibilizer in combination with varying amounts of TESPT, VP Si-363 and NXT.

The addition of ENR-51 as compatibiizer in the silica-filled NR compounds increases the chemically bound rubber but decreases the physically bound rubber, attributed to generation of interactions via hydrogen bonding and/or chemical bonding during mixing at high temperature between the epoxy groups of ENR and the silanol groups of silica. The chemically bound rubber contents of the compounds clearly increase with increasing amount of TESPT, NXT and VP Si-363, respectively, indicating that remaining silanol groups further interact with the additional functional groups of the silanes, leading to a further increase of silica-rubber interactions.

The use of TESPT gives the highest chemically bound rubber content and the lowest physically bound rubber content, while the VP Si-363 gives the lowest chemically bound rubber and the highest physically bound rubber of the three silanes. TESPT has a bis-(triethoxy-)-structure in which there are more ethoxy groups available to react with the silanol groups of silica. Moreover, the tetrasulfide part may introduce premature scorch during mixing at high temperature. ${ }^{[18]} \mathrm{NXT}$ has a triethoxy group and the lowest molecular weight among the three types of silane used in this work, and so will have more ethoxy 
groups available for silanization compared with VP Si-363 which has only 1 ethoxy group in a molecule and the highest molecular weight due to the bulky long alkoxy substituents, as shown in Table 5.1. As reported by Klockmann ${ }^{[16]}$, for VP Si-363 the silica-silane reaction proceeds via the ethoxy group, while the long dialkoxy groups with polyglycol parts leads to fast adsorption on the silica surface and increases hydrophobation. This results in the lowest chemically bound rubber content, but the highest physically bound rubber content of the three silanes.

\subsubsection{Cure properties}

From the cure curves of the silica-filled NR compounds with different types of silane coupling agent in Figures 5.3(a-c), the compound without any compatibilizer shows the highest torque prior to real vulcanization due to filler flocculation, causing the reformation of silica agglomerates that hinder chain movement. It is well-known that reagglomeration of silica or filler flocculation in the filled compounds may take place in the beginning of the vulcanization reaction when the compounds are heated and less viscous. ${ }^{[19-21]}$ The rate of the filler flocculation in the rubber matrix depends on filler loading, polymer matrix type, viscosity, mixing history and temperature. ${ }^{[19]}$ Increasing the temperature for vulcanization leads initially to a decrease of polymer matrix viscosity and increase of the filler diffusion coefficient causing an easier movement of filler particles/aggregates to quickly form filler-filler networks under the shearing condition. ${ }^{[20]}$ The silica-filled NR compounds with optimal TESPT content, i.e. $8.6 \mathrm{wt} \%$ relative to silica, shows no occurrence of flocculation due to a good interfacial interaction between silica and the rubber phase through the silanization reaction that shields the free silanol groups on the silica surface. ${ }^{[21]}$ The eventual premature crosslinks introduced by sulfur out-of TESPT also help to suppress the flocculation in silica-filled NR compounds. ${ }^{[18]}$ The use of ENR-51 at $7.5 \mathrm{phr}$ as compatibilizer substantially reduces the silica flocculation in the silica-filled NR compound but the phenomenon is still clearly observed in the induction period of the cure curves, indicating that free silanol groups are still present in the compound at a larger amount compared with TESPT. The ENR compatibilized compounds also show the poorest cure rate, as observed by a low slope of the cure curve in Figure 5.3, the longest optimum cure time as seen in Figure 5.4 (a), and the lowest torque difference in Figure 5.4 (b). This indicates poor cure behavior in the presence of ENR-51 alone as compatibilizer. 

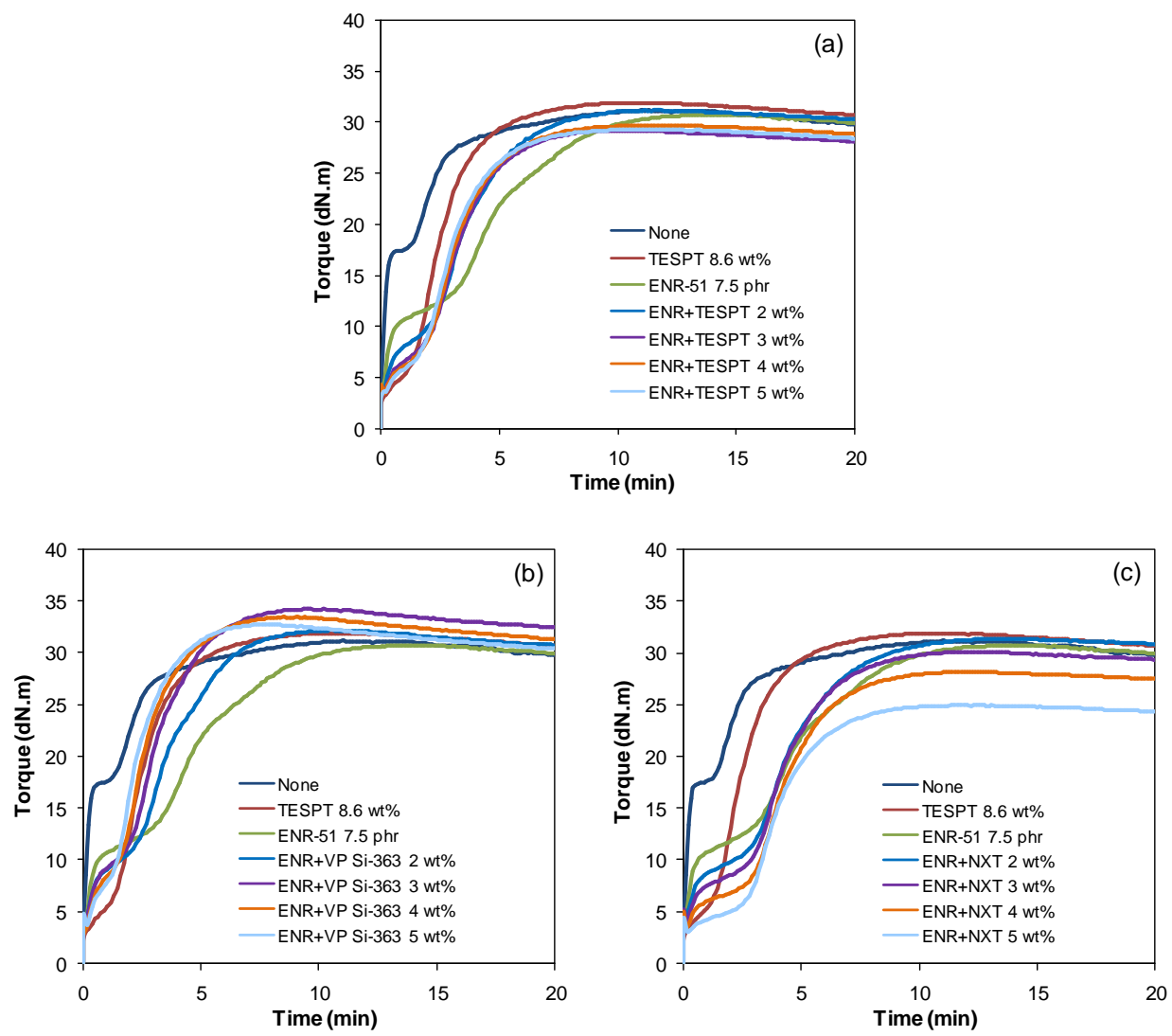

Figure 5.3 Cure curves of silica-filled NR compounds with $7.5 \mathrm{phr}$ of ENR-51 as compatibilizer and varying amounts of different silanes; TESPT (a), VP Si-363 (b) and NXT (c), in comparison with that of the compounds without compatibilizer and with reference TESPT. 

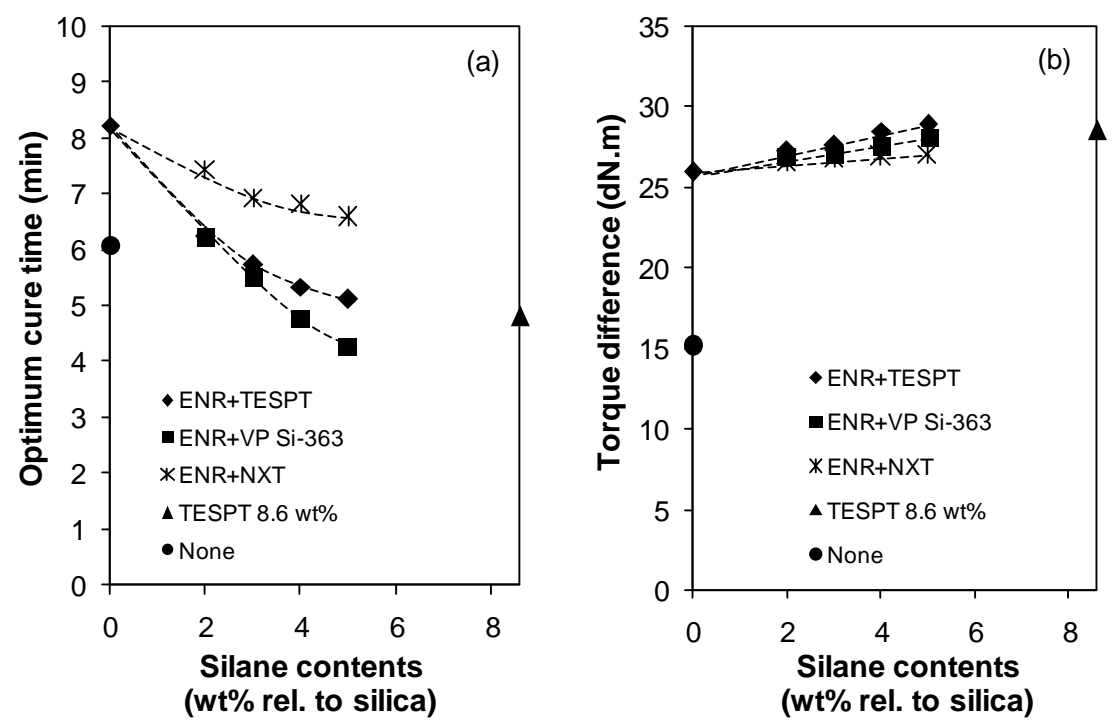

Figure 5.4 Optimum cure time (a) and torque difference (b) of silica-filled NR compounds with $7.5 \mathrm{phr}$ of ENR-51 as compatibilizer in combination with varying amounts of TESPT, VP Si-363 and NXT.

The addition of silane coupling agents in combination with ENR-51 into the silicafilled NR compounds reduces the silica flocculation by an additional increase of chemical interfacial interactions between the silica and rubber phases, as seen in the increased bound rubber content in Figure 5.2. In Figure 5.3(a), the ENR/TESPT compounds show a decrease of minimum torque with increasing TESPT concentrations, to finally reach the same level as that of the compound with reference TESPT. Shorter scorch and optimum cure times (Figure 5.4(a)) are also observed when TESPT was added to the compound with ENR as compatibilizer. This is due to a further decrease of free silanol groups by the silanization reaction on the silica. In the presence of TESPT, the vulcanization reaction is also accelerated by its sulfur donor effect. The results for mercaptosilane VP Si-363, as shown in Figure 5.3(b), display a reduction of silica flocculation with increasing VP Si-363 content, but the minimum torques of the compounds are still higher than for the reference TESPT. This indicates a lesser extent of filler-rubber interactions due to the fact that the VP Si-363 molecule has two bulky alkoxyl groups with only one ethoxy group to react with the silanol groups of silica leading to less silanization when compared with TESPT. On an equal weight basis, the higher molecular weight of VP Si-363 also results in lower number of molecules and functional groups for the reaction. The bulky substituents prevent the "scorchiness" of the mercapto group by steric hindrance ${ }^{[16]}$, but this mercapto group 
provides a more efficient coupling with rubber during the vulcanization reaction when compared with the compounds with ENR and TESPT. This is also observed by the shorter scorch and optimum cure times of the compounds containing VP Si-363 in Figure 5.3 (a-b) and Figure 5.4(a). Generally, compounds with TESPT have a much better scorch safety than compounds with a normal mercaptosilane such as $\gamma$-mercapto propyltriethoxysilane (MPS) due to the steric hindrance of the bifunctional groups of tri-ethoxysilyl-propyl that surround the tetrasulfide part in TESPT, leading to a less active sulfur moiety for coupling and vulcanization. ${ }^{[11]}$

Increasing NXT content shows a gradual decrease of silica flocculation and minimum cure torques as shown in Figure 5.3 (c), because of improvements in silicarubber interactions and the increase of plasticization fragments (i.e. octanoyl part) of the NXT molecule. This aliphatic tail also provides hydrophobicity and improves the silica dispersion causing a lowering in the minimum torque, corresponding to the compound viscosity. The compounds with NXT show outstanding long scorch times, i.e. better scorch safety compared to the ones with TESPT. This is due to the mercaptan group blocked by the long aliphatic blocking group in NXT structure, as shown in Table 5.1, that reduces the silane-NR reactivity during processing, so that the silane-NR coupling occurs only during vulcanization. $^{[14]}$

Figure 5.4(b) shows the cure torque difference $\left(\mathrm{M}_{\mathrm{H}}-\mathrm{M}_{\mathrm{L}}\right)$ which is closely related to crosslink density in the materials, including both chemical bonds and interactions. It is clearly seen that the use of all compatibilizer types strongly increases the cure torque difference compared to the non-compatibilized one, and the use of the silane containing a higher sulfur rank, i.e. TESPT, gives the highest torque differences, indicating more crosslinks in the vulcanizates. 


\subsubsection{Tensile properties}
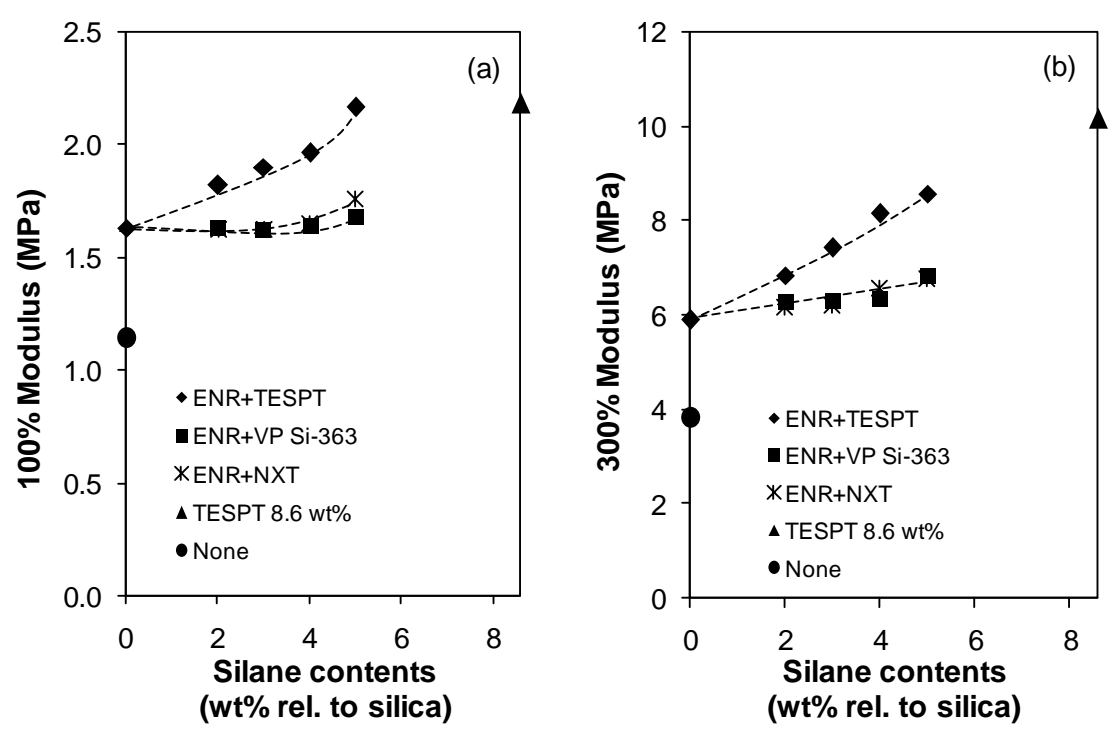

Figure 5.5 Moduli at $100 \%$ (a) and $300 \%$ (b) elongations of silica-filled NR vulcanizates with 7.5 phr of ENR-51 as compatibilizer in combination with varying amounts of TESPT, VP Si-363 and NXT.

The silica-filled NR vulcanizates with ENR/TESPT combinations as compatibilizers show a remarkable increase of the $100 \%$ and $300 \%$ moduli with increasing TESPT content, as shown in Figures 5.5(a) and (b), whereas the ENR/VP Si-363 and ENR/NXT combinations show only a small change in tensile modulus with increasing silane contents. The substantially higher modulus of the vulcanizates with TESPT is caused by the highest filler-rubber interactions in the silica-filled compounds as determined by the bound rubber content: Figure 5.2, and the higher crosslink density resulting from sulfur released from the TESPT molecule. The VP Si-363 and NXT have only 1 sulfur atom in the molecule and cannot donate sulfur to the system. Furthermore, when considering the molecular structures and molecular weights of the silanes, VP Si363 and NXT provide lower amounts of ethoxy groups to bond to the silanol groups of silica when compared to TESPT. 


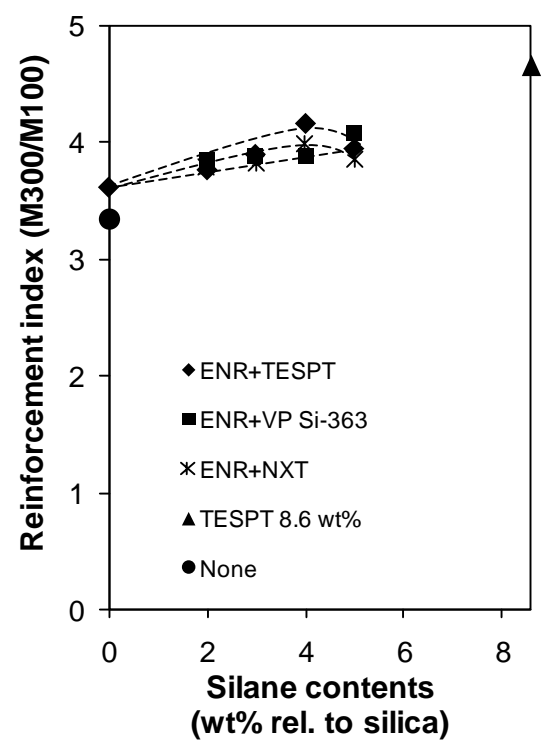

Figure 5.6 Reinforcement index of silica-filled NR vulcanizates with 7.5 phr of ENR-51 as compatibilizer in combination with varying amounts of TESPT, VP Si-363 and NXT.

The ratio of tensile moduli at $300 \%$ to $100 \%$ elongation is often used for determining the reinforcement index of silica-filled rubber vulcanizates. As shown in Figure 5.6, the reinforcement index of the silica-filled NR vulcanizate with reference TESPT only is remarkably higher than that of all other vulcanizates, and the compatibilization by using ENR results only in a slightly higher reinforcement index than the fully non-compatibilized one. The introduction of silanes to combine with ENR increases the reinforcement index somewhat, but not to the level of TESPT alone. The reinforcement index relates to fillerrubber interactions which are indicated by the chemically bound rubber content as shown in Figure 5.2(a). A higher silica-rubber interaction increases the modulus of vulcanizates due to more restriction of chain movement. For the ENR/silane combination, the use of TESPT silane gives the highest reinforcement index among the three types of silanes studied and an optimum value is observed at $4.0 \mathrm{wt} \%$ of TESPT relative to silica. 

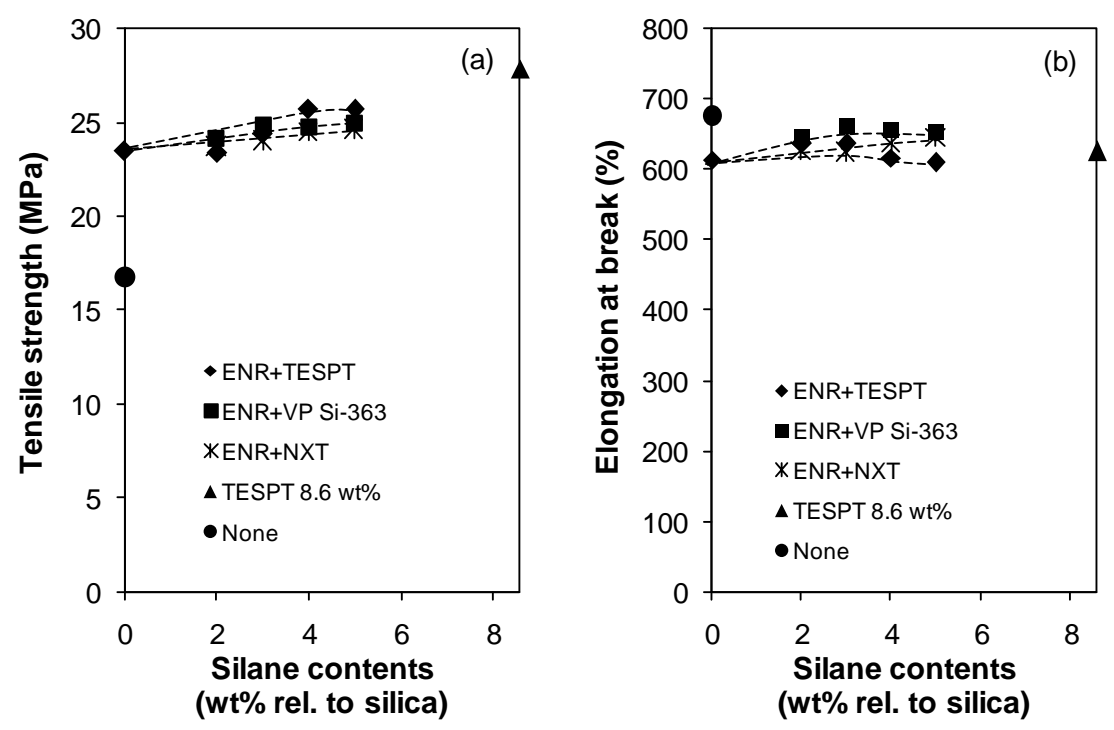

Figure 5.7 Tensile strength (a) and elongation at break (b) of silica-filled NR vulcanizates with $7.5 \mathrm{phr}$ of ENR-51 as compatibilizer in combination with varying amounts of TESPT, VP Si-363 and NXT.

Tensile strength and elongation at break of the silica-filled NR vulcanizates with ENR-51/silane combinations as compatibilizer are increased slightly with increasing silane concentrations, as shown in Figure 5.7. The use of 7.5 phr of ENR-51 as compatibilizer apparently leaves some free silanol groups in the system and so the addition of just a small amount of silane coupling agent can further enhance the interaction between the silica and rubber phases through the silanization reaction. Tensile strength of the silicafilled vulcanizates with optimal content of TESPT is the highest, and the ENR/TESPT combination gives a higher tensile strength than the use of the other two types of silane. The ENR/TESPT system shows an optimum tensile strength when $4.0 \mathrm{wt} \%$ of TESPT relative to silica was used, which is still inferior to that of the reference compound with TESPT alone. Overall, the use of different silanes in combination with 7.5 phr of ENR-51 results in only small differences in tensile strength and elongation at break, where both properties are only slightly increased with increasing silane loadings. 


\subsubsection{Effect of extra sulfur on the properties of silica-filled NR compatibilized with the ENR51/silane combination}

Due to the structural differences between the three silane types as shown in Table 5.1, the amount of total sulfur in the compounds which affects the network formation is therefore different. By taking the compound with TESPT of $8.6 \mathrm{wt} \%$ relative to silica as a reference, corrections are applied for the other compounds with extra elemental sulfur added together with other curatives on a two-roll mill in the second step of mixing. The silica-filled NR compounds for this study are compatibilized with ENR-51 at $7.5 \mathrm{phr}$ and silane at $4.0 \mathrm{wt} \%$ relative to silica, i.e. $2.2 \mathrm{phr}$ in the formulation: Table 5.3. The three types of silane coupling agents, i.e. TESPT, VP Si-363 and NXT, are used with a sulfur correction to compensate for the sulfur content in the reference compound with TESPT alone. The properties are reported in comparison with those without compatibilizer, with reference TESPT (i.e. $8.6 \mathrm{wt} \%$ rel. to silica), and with $7.5 \mathrm{phr}$ of ENR-51 only.

\subsubsection{Mooney viscosity}

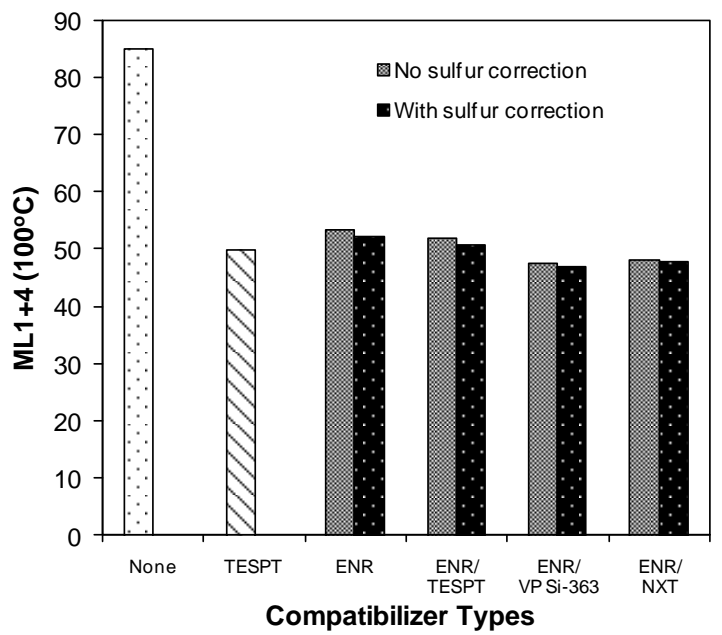

Figure 5.8 Mooney viscosities of silica-filled NR compounds with different combinations of ENR-51 (7.5 phr)/silanes (2.2 phr) without and with extra sulfur, compared to the ones without compatibilizer, resp. with reference TESPT only and with ENR-51 alone.

As previously seen in Figure 5.1, and again in Figure 5.8, the silica-filled NR compound without compatibilizer shows the highest Mooney viscosity, and the incorporation of compatibilizers at their optimum amount drastically reduces the Mooney 
viscosity to similar levels as results of enhanced filler-rubber interactions and improved silica dispersion. With 7.5 phr of ENR-51 and 4 wt\% of VP Si-363 and NXT relative to silica content, the compounds show slightly lower Mooney viscosities compared with the ones with TESPT 8.6 wt\% relative to silica, with ENR and with the ENR/TESPT combination. The long blocking group of NXT and the polymeric alkoxy substituents of VP Si-363 should help in shielding the silica surface, promote more hydrophobicity, aid silica dispersion, and improve processability. The compounds with and without sulfur correction show more or less the same level of Mooney viscosities as the extra sulfur was only introduced in the second step of mixing on the two-roll mill. This extra sulfur is therefore expected to take part in the vulcanization only and play no role in the mixing stage.

\subsubsection{Cure properties}
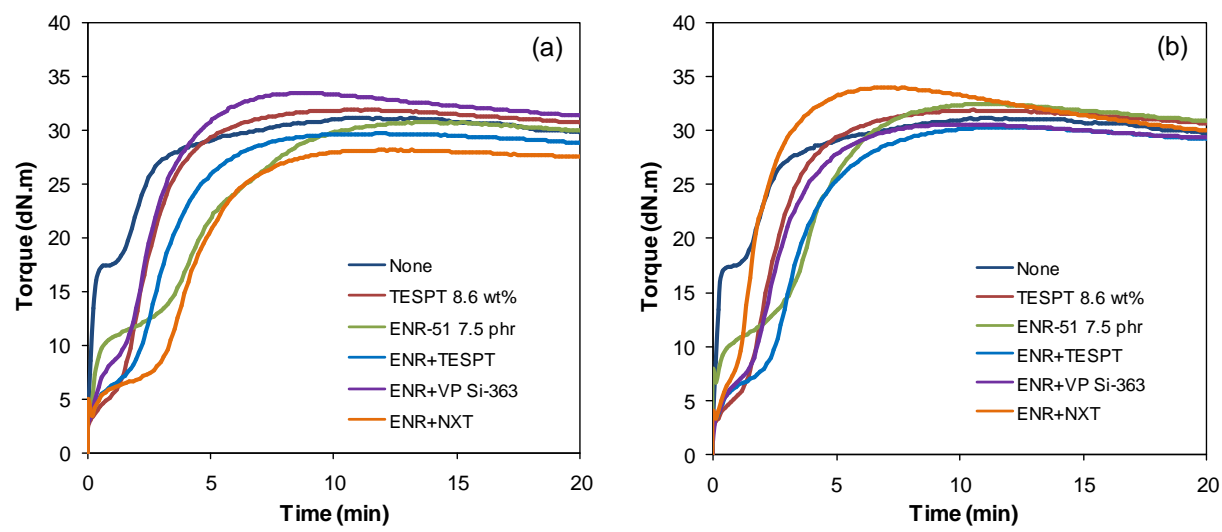

Figure 5.9 Cure characteristics of silica-filled NR compounds with different combinations of ENR-51 (7.5 phr)/silanes (2.2 phr) without (a) and with (b) extra sulfur, compared to the ones without compatibilizer, resp. with reference TESPT only and with ENR-51 alone. 

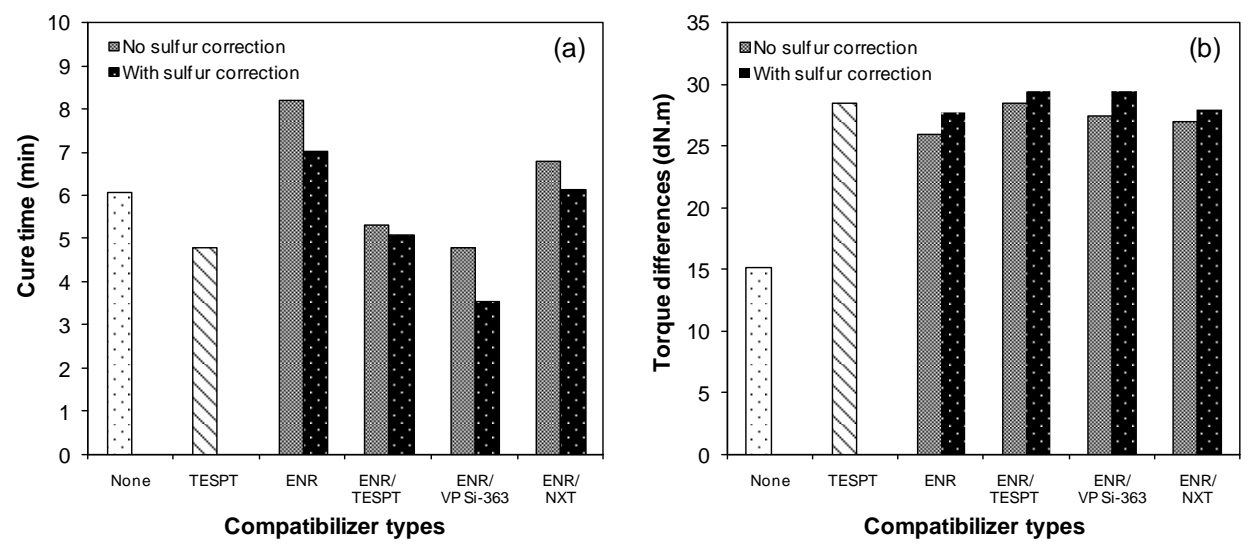

Figure 5.10 Cure time (a) and torque differences (b) of silica-filled NR compounds with different combinations of ENR-51 (7.5 phr)/silanes (2.2 phr) without and with extra sulfur, compared to the ones without compatiblizer, resp. with reference TESPT only and with ENR-51 alone.

When extra sulfur was added into the silica-filled NR compounds, all of the compounds show an increase of maximum cure torques and faster cure times as seen in Figures $5.9(\mathrm{a}, \mathrm{b})$ and Figures $5.10(\mathrm{a}, \mathrm{b})$. The correction of the compounds with elemental sulfur relative to the sulfur contained in the reference TESPT system leads to an increase of crosslink density in the rubber matrix and consequently to the increase of maximum cure torques and torque differences, as shown in Figure 5.10(b). It was reported that increasing sulfur content in the rubber compounds increases the maximum torque due to the increase in total crosslink density, increased glass transition temperature $\left(T_{g}\right)$ and decreased fractional free volume of the material. ${ }^{[22]}$ With sulfur correction, the minimum cure torques show only a little change, in accordance with the Mooney viscosity results in Figure 5.8. On comparing between the three silane types that were used in combination with ENR-51, the use of VP Si-363 gives the fastest scorch and cure times due to the reactive mercapto function, and the cure curves show a strong reversion after the optimum cure. The use of NXT gives the best scorch safety, while the use of TESPT shows results somewhere in between. 


\subsubsection{Tensile properties}
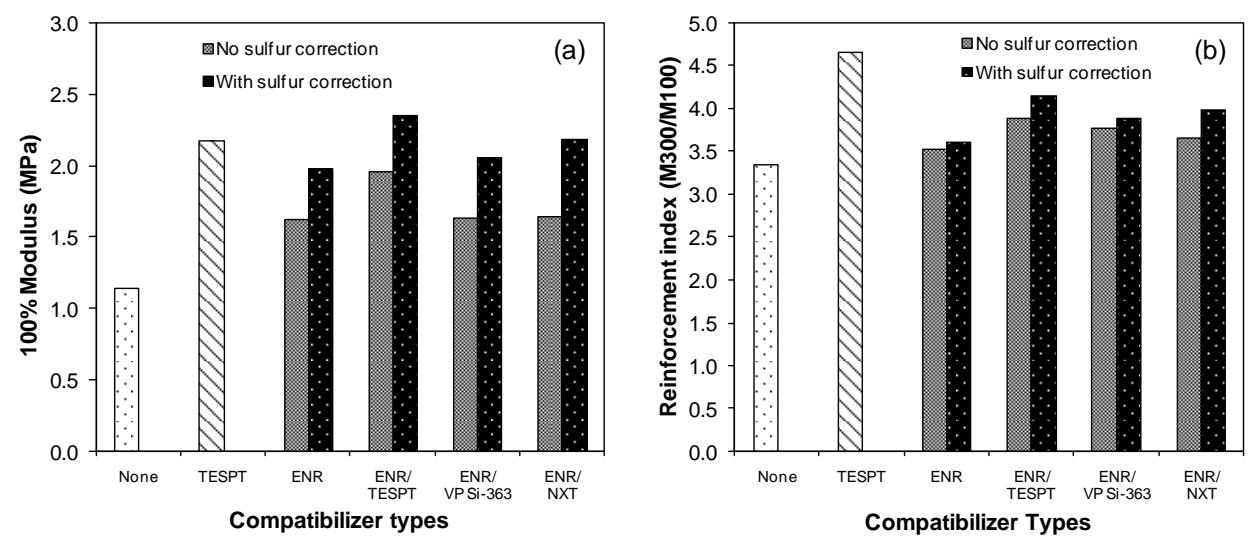

Figure 5.11 100\% Modulus (a) and reinforcement index (b) of silica-filled NR vulcanizates with different combinations of ENR-51 (7.5 phr)/silanes (2.2 phr) without and with extra sulfur, compared to the ones without compatiblizer, resp. with reference TESPT only and with ENR-51 alone.

The tensile modulus at $100 \%$ elongation and reinforcement index of the silicafilled NR vulcanizates with different types of compatibilizers are shown in Figures 5.11(a) and (b). For the combination system, the use of ENR-51/TESPT shows the highest $100 \%$ modulus and reinforcement index, while the ENR-51/VP Si-363 and ENR-51/NXT show similar properties. All the compatibilized compounds show very much better moduli than the compounds without compatibilizer and the benchmark TESPT gives outstanding properties. With the correction of sulfur content in the silica-filled NR compounds relative to the reference TESPT compound, a significant increase of tensile modulus of the vulcanizates is obtained due to an increase in crosslink density. The reinforcement index is also slightly increased by sulfur correction. 

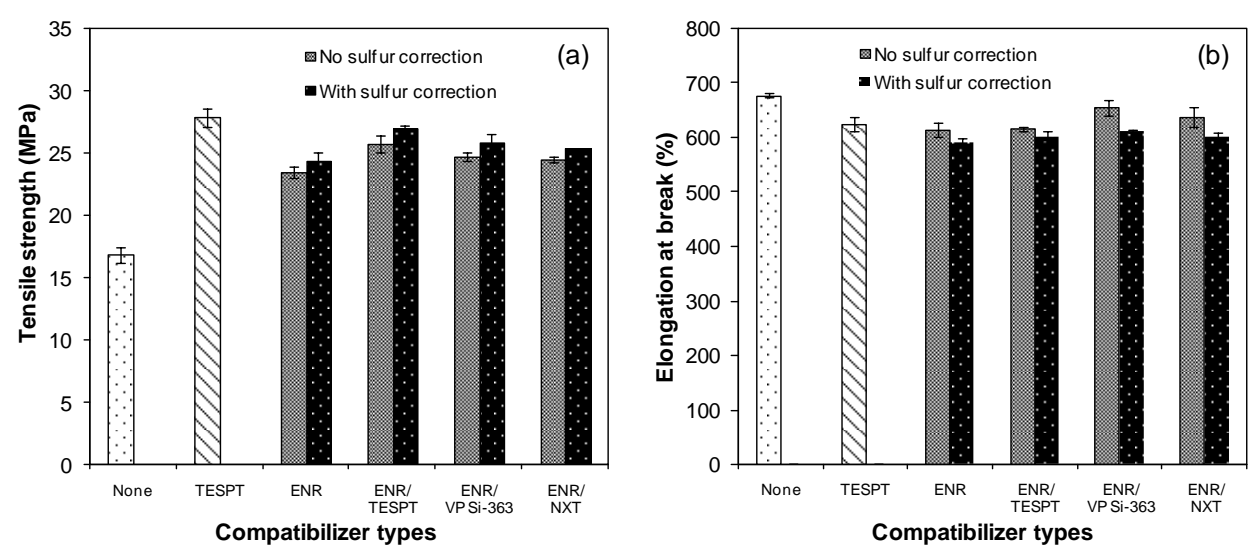

Figure 5.12 Tensile strength (a) and elongation at break (b) of silica-filled NR vulcanizates with different ENR-51 (7.5 phr)/silanes (2.2 phr) combinations without and with extra sulfur, compared to the ones without compatiblizer, with reference TESPT only and with ENR-51 alone.

Tensile strength of the vulcanizates is also improved somewhat by applying the sulfur correction relative to the reference TESPT compound as shown in Figure 5.12(a). In accordance with the modulus, the use of TESPT together with ENR-51 gives the highest tensile strength among the three different ENR/silane combinations due to the number of ethoxy groups for interaction with the silanol groups on the silica surface to increase the silica-filler interactions, and the sulfur donated from TESPT molecules to contribute to network formation in the vulcanizates. When compared to the compounds that contain TESPT and the same total sulfur content, the use of TESPT $8.6 \mathrm{wt} \%$ relative to silica gives a higher $300 \%$ modulus and tensile strength than the use of ENR/TESPT with sulfur correction. This may indicate a more effective use of sulfur released from the TESPT molecules during the mixing at high temperature, when compared to the corrected sulfur that was added on the cold two-roll mill. When the modulus of a vulcanizate is increased due to higher network density, causing a higher stiffness, elongation at break decreases as shown in Figure 5.12(b). 


\subsubsection{Dynamic mechanical properties}
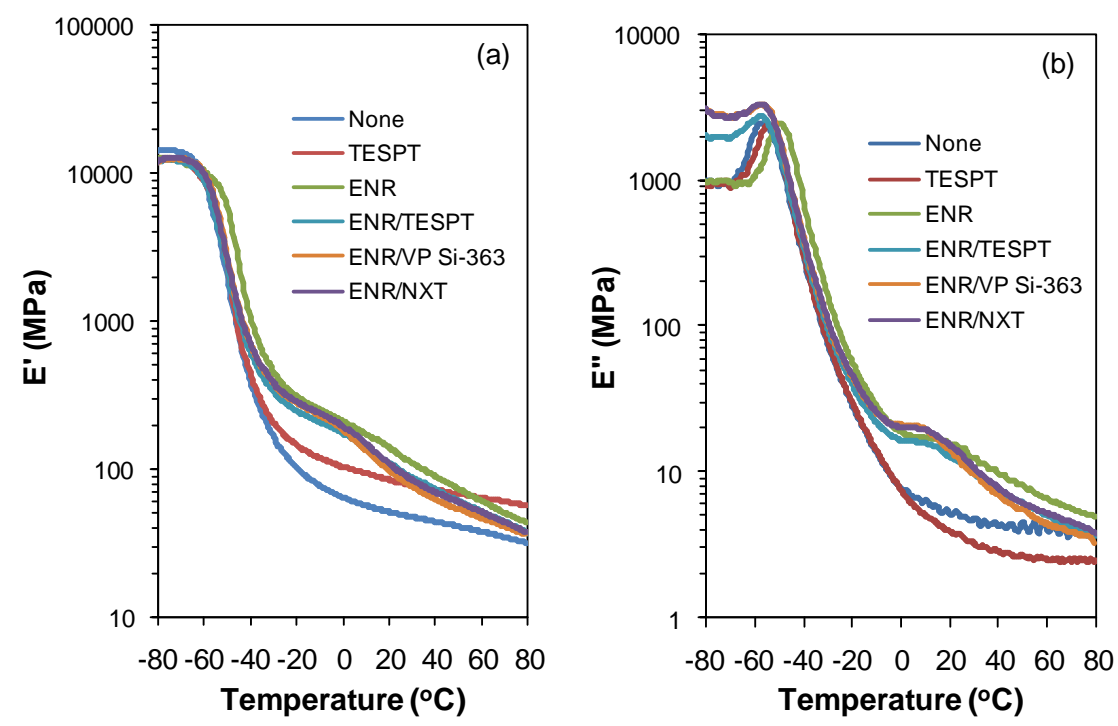

Figure 5.13 Storage (a) and loss (b) moduli of silica-filled NR vulcanizates compatibilized with 7.5 phr of ENR-51 and 4.0 wt\% of silanes relative to silica, with sulfur compensation, in comparison with those vulcanizates without compatibilizer, resp. with reference TESPT and with ENR-51 alone.

Storage moduli $\left(E^{\prime}\right)$ of the silica-filled NR vulcanizates containing different compatibilizers show only small differences in the glassy region, where the molecular motions are largely restricted. However, the vulcanizates do show their different behaviors in the rubbery region, which is determined by the segmental mobility of the rubber chains. When compared the silica-filled NR with only TESPT and without compatibilizer, the silica/TESPT system shows higher storage modulus ( $\left.E^{\prime}\right)$ but lower loss modulus ( $\left.E "\right)$ after the glass-to-rubber transition as shown in Figures 5.13(a) and (b). As previously discussed, the silica/TESPT compound has the strongest filler-rubber interactions by its highest chemically bound rubber content in Figure 5.2(a) and an increase of crosslink points through silane bridges. This vulcanizate therefore shows higher elastic modulus and better elasticity that leads to lower energy loss under dynamic conditions. The silicafilled NR without compatibilizer that has the highest filler-filler interaction or Payne effect; Figure 5.1(a), leads to less elasticity of the material as the rubber being trapped in the filler-filler network and low interfacial interaction between the silica and rubber phases causes loss of energy as dissipated in heat. ${ }^{[23]}$ The incorporation of ENR-51 and ENR- 
$51 /$ silanes combinations introduce the secondary transition in the range of -10 to $10^{\circ} \mathrm{C}$ as observed in both the storage and loss moduli curves. This second transition is due to the presence of the second polymer ENR-51, which has a higher $T_{g}$ than NR and the damping behavior of the ENR in the $T_{g}$-transition range. The presence of two $T_{g}$ 's confirms that NR and ENR-51 are basically immiscible.

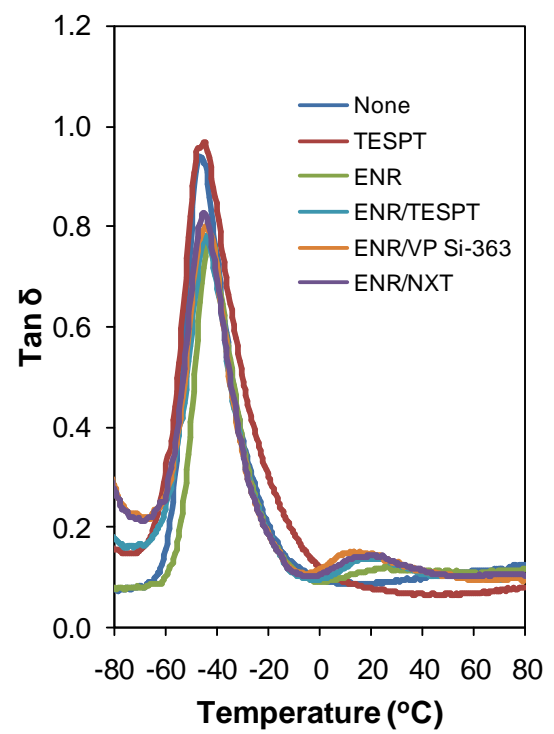

Figure 5.14 Loss tangent (tan $\delta$ ) of silica-filled NR vulcanizates compatibilized with $7.5 \mathrm{phr}$ of ENR-51 and 4.0 wt\% of silanes relative to silica with sulfur compensation, in comparison with those vulcanizates without compatibilizer, resp. with reference TESPT only and with ENR-51 alone.

The results of tan $\delta$ in Figure 5.14 show that the silica-filled NR with TESPT has the highest intensity of tan $\delta$ peak due to the improved of silica dispersion and less rubber trapped in the silica network, leading to more rubber segments involved in the polymer segmental relaxation in the glassy region. ${ }^{[24]}$ The addition of ENR as compatibilizer in the silica-filled NR decreases the intensity of tan $\delta$ at peak, as also shown in the values in Table 5.4. This reduction is caused by the silica-ENR interactions and intermolecular interactions between epoxy groups of adjacent ENR molecules, which results in a restriction of polymer chain relaxations and an increase in the material stiffness. These results are in accordance with the tan $\delta$ curves as previously shown in Figures 3.12 and 4.10. The addition of silane coupling agents together with ENR-51 increases the $(\tan \delta)_{\max }$ values slightly when compared to the vulcanizate with only ENR-51, as summarized in 
Table 5.5, because of a further improvement of filler dispersion and less occluded rubber. Among the different compatibilizer types, the tan $\delta$ peak of the TESPT-compound is broader towards the rubbery region compared to that of the others as a result of a greater extent of filler-rubber interactions in this material.

The second glass transition peak corresponding to ENR-51 is clearly observed for the compounds that are compatibilized with either ENR only or with ENR/silane. According to Manna et al. ${ }^{[25]}$, epoxidized natural rubber with $50 \mathrm{~mol} \%$ of epoxy groups and 60 phr of silica has a $\mathrm{T}_{\mathrm{g}}$ of about -3 to $1^{\circ} \mathrm{C}$. It is observed in Figure 5.14 that in the presence of silane coupling agents, the second tan $\delta$ peak intensity is increased and the peak position shifted toward lower temperature in comparison with ENR-51 alone. In the systems with ENR/silane combinations, the silica-silane interactions are promoted together with epoxide-silica interactions. In this case, there is competition of more free epoxide groups in the ENR compared to the silica-filled NR with only ENR as compatibilizer. In the combination system, ENR therefore exhibits its characteristic peak in a more prominent manner. For the compound with only ENR as compatibilizer, the second $\tan \delta$ peak is broader and less pronounced because of more interactions between epoxide groups of ENR and silanol groups on the silica, leading to a weaker characteristic peak of ENR. This observation of the change of $\tan \delta$ peak of epoxidized natural rubber as compatibilizer in silica-filled NR due to the level of filler-rubber interactions was also reported by Saramolee et al. ${ }^{[26]}$

The $\mathrm{T}_{\mathrm{g}}$ values taken at both $\tan \delta$ and loss modulus peaks are summarized in Table 5.5 together with the $\tan \delta$ values at different temperatures. On comparing with the non-compatibilized material, the $\mathrm{T}_{\mathrm{g}}$ 's of the vulcanizates with compatibilizers shift to higher temperature slightly, due to an increase of silica-rubber interactions that restrict the rubber segmental relaxations in the transition region.

Table 5.5 $\mathrm{T}_{\mathrm{g}}$ and tan $\delta$ values at different positions taken from Figure 5.14.

\begin{tabular}{lccccc}
\hline \multirow{2}{*}{ Compatibilizer types } & \multicolumn{3}{c}{$\mathbf{T}_{\mathbf{g}}\left({ }^{\circ} \mathrm{C}\right)$} & \multicolumn{3}{c}{ Values of Tan $\delta$} \\
\cline { 2 - 6 } & at tan $\delta$ peak & at E" peak & at peak & at $5{ }^{\circ} \mathrm{C}$ & at $60^{\circ} \mathrm{C}$ \\
\hline Without compatibilizer & -47 & -58 & 0.94 & 0.09 & 0.11 \\
TESPT & -45 & -54 & 0.97 & 0.10 & 0.07 \\
ENR-51 & -44 & -52 & 0.77 & 0.09 & 0.11 \\
ENR-51+TESPT+S & -45 & -57 & 0.79 & 0.12 & 0.10 \\
ENR-51+VP Si-363+S & -45 & -57 & 0.80 & 0.13 & 0.09 \\
ENR-51 +NXT+S & -45 & -57 & 0.82 & 0.11 & 0.10 \\
\hline
\end{tabular}



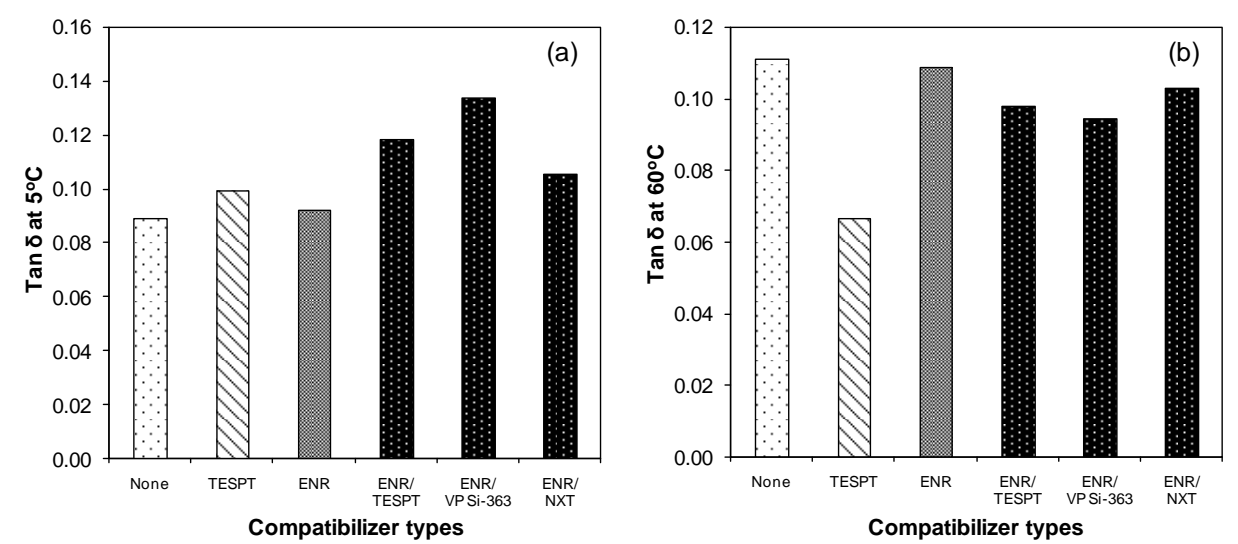

Figure 5.15 Tan $\delta$ values at $5^{\circ} \mathrm{C}$ (a) and $60^{\circ} \mathrm{C}$ (b) of silica-filled NR vulcanizates with different combinations of ENR-51 (7.5 phr)/silanes (2.2 phr) with sulfur correction, compared to the ones without compatiblizer, resp. with reference TESPT only and with ENR-51 alone.

For the tan $\delta$ values at $5^{\circ} \mathrm{C}$ and $60^{\circ} \mathrm{C}$, the results are plotted in Figure 5.15. According to UNECE regulation no. $117^{[27]}$, wet grip grading of normal tires should be tested in the temperature range of $5-35^{\circ} \mathrm{C}$. Figure 5.15 (a) shows the $\tan \delta$ values at $5^{\circ} \mathrm{C}$ of the silica-filled vulcanizates containing different compatibilizers and without. The use of ENR-51 together with silane coupling agents gives the highest $\tan \delta$ values in the temperature range of $5-35^{\circ} \mathrm{C}$ which is in the range of the second glass transition associated with the ENR, when compared to the use of TESPT, ENR only and no compatibilizer, respectively. The different levels of interactions between the epoxide functions and silanol groups on the silica when ENR is used alone or with silane, may cause differences in the segmental relaxation behavior of the NR and affect the peak intensity. The increased $\tan \delta$ at low temperature indicates a better wet grip of tire compounds.

On the other hand, the tan $\delta$ value at high temperature, commonly in the range of $40-80^{\circ} \mathrm{C}$ indicates tire rolling resistance. As shown in Figure 5.15(b), the incorporation of ENR either with or without silane results in high tan $\delta$ values at $60^{\circ} \mathrm{C}$ which are just slightly lower than that of the compound without compatibilizer. This damping property is again related to the $T_{g}$ of ENR-51. The high mol\% of epoxide groups on the ENR introduces chain stiffness and lower elasticity. Moreover, the different polarity of the two rubber phases leads to blend incompatibility which causes an energy loss at their interface under dynamic deformation. Among the different compatibilizers studied, the use of conventional 
TESPT in silica-filled NR compounds provides a remarkably low tan $\delta$ at $60^{\circ} \mathrm{C}$, indicating superior low rolling resistance for tire compounds.

\subsubsection{Surface topography}

Tensile fractured surfaces of silica-filled NR vulcanizates without compatibilizer, with TESPT, with only ENR-51 and with ENR/silane combinations are shown in Figures 5.16(a) - (i). As previously seen in Figure 4.13 and discussed in Chapter 4, the lowest tensile strength due to poor filler-rubber interactions of the vulcanizate without compatibilizer results in a rather smooth fracture surface, whereas the fracture surface of the silica-filled rubber vulcanizate with TESPT shows a rough surface with many tear lines because of the strong silica-rubber interactions and bonding, leading to the high mechanical strength of the material. ${ }^{[28]}$ The fracture surface of the vulcanizate with ENR as compatibilizer also shows tear lines, but less than that of the reference TESPT compound. Therefore, the use of silane in addition to ENR as compatibilizer may reduce silica dewetting in the rubber matrix, and improve interfacial adhesion between filler and rubber. 

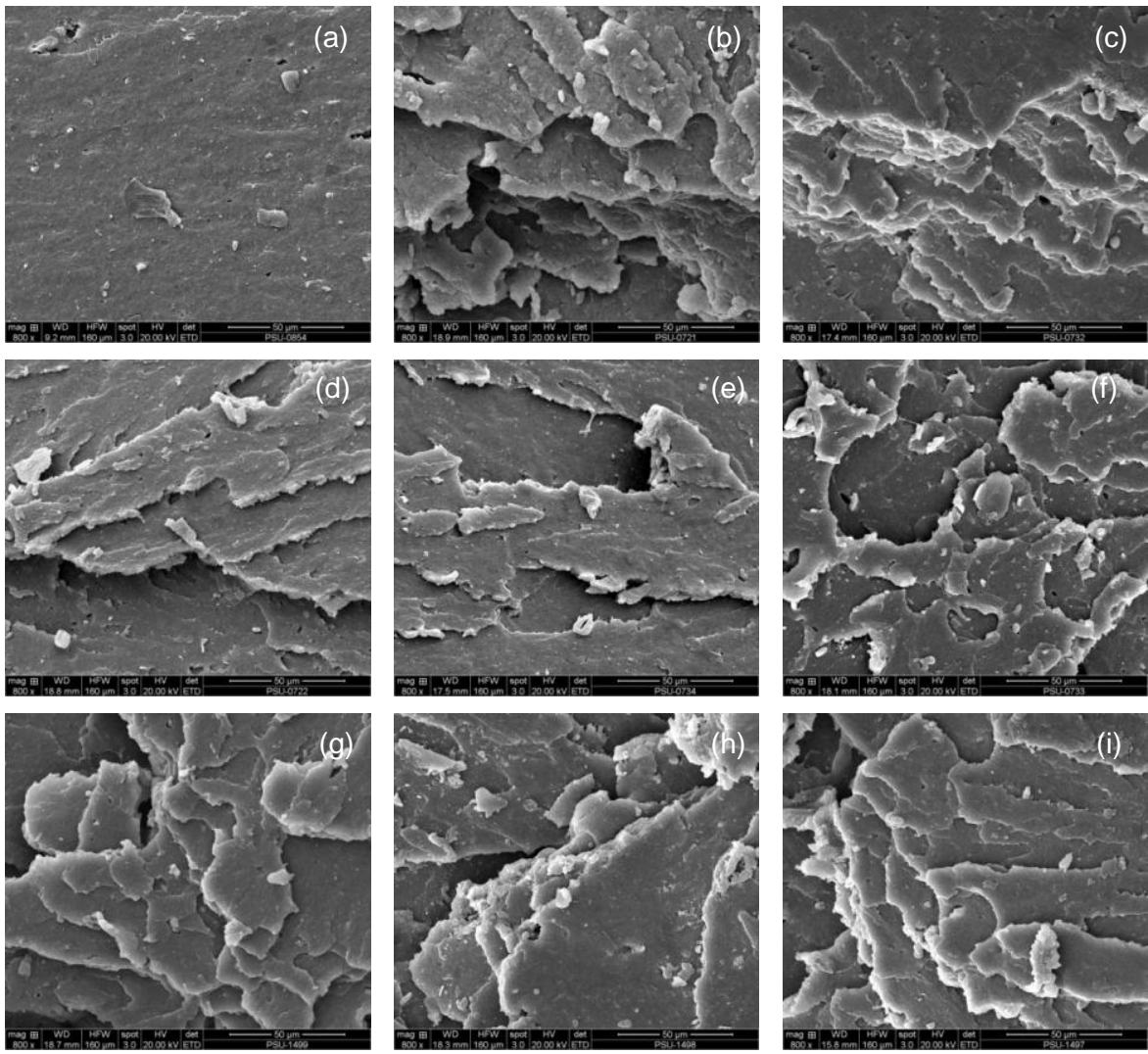

Figure 5.16 Tensile fractured surfaces of silica-filled NR vulcanizates with different types of compatibilizers; upper row: reference compounds without compatibilizer (a), with TESPT (b), with ENR-51 (c); middle row: compounds without extra sulfur: ENR/TESPT (d), ENR/VP Si-363 (e), ENR/NXT (f); lower row: compounds with extra sulfur: ENR/TESPT/S (g), ENR/VP Si-363/S (h), ENR/NXT/S (i).

Figures 5.16(d)-(f) show the failure surfaces of the silica-filled NR with ENR/silane combinations without sulfur correction and Figures 5.16(g)-(i) with sulfur correction. All of them show more tear lines on the fracture surface compared to the silicafilled NR without compatibilizer in Figure 5.16(a), but a less complicated tear line pattern compared with that of the vulcanizate with TESPT; Figure 5.16(b). The use of ENR-51 in combination with silane coupling agents leads to an improvement of silica-rubber interaction, and the addition of extra sulfur that increases the crosslink density in the rubber vulcanizate further increases the tensile strength of the materials. The increase of tensile strength creates more surface roughness due to ductile failure. Among the three 
types of silane coupling agents used together with ENR-51 as compatiblizer, there is no clear trend seen between the two sets of vulcanizates without and with extra sulfur.

\subsection{Conclusions}

The properties of silica-filled NR compounds compatibilized with $7.5 \mathrm{phr}$ of ENR51 can be further enhanced by the addition of silane coupling agents at a smaller amount compared to the normal use of TESPT. The study on the use of three types of silane; TESPT, NXT and VP Si-363, at varying amounts in the range of 2 to $5 \mathrm{wt} \%$ relative to silica together with $7.5 \mathrm{phr}$ of ENR-51 shows, that the addition of silane reduces the compound viscosity slightly but clearly enhances the chemically bound rubber content and cure properties. Among the 3 different ENR-51/silane combinations, the use of ENR/TESPT gives the highest chemically bound rubber content and tensile properties, whereas the ENR/NXT combination gives higher chemically bound rubber content but similar tensile properties compared to the ENR/VP Si-363 system. The optimum tensile strength is obtained when $7.5 \mathrm{phr}$ of ENR-51 with TESPT at $4.0 \mathrm{wt} \%$ relative to silica are used. The better improvement of the overall properties by the use of TESPT over the other two types of silanes is due to the bis-(triethoxysilyl)- and tetrasulfide-functional groups that provide more ethoxy groups relatively to react with the silanol groups on the silica surface, and the reactive free sulfur released to involve in network formation. When the amount of silane coupling agent is fixed at $4.0 \mathrm{wt} \%$ relative to silica in combination with 7.5 phr of ENR-51 as compatibilizers in the silica-filled NR compound, the addition of extra sulfur to correct the total sulfur content relative to the reference compound with TESPT leads to increases of modulus and tensile strength while elongation at break decreases. The enhanced interactions and bonding between the silica and rubber phases by means of compatibilizers, either without or with sulfur correction, shift the $T_{g}$ of the silica-filled NR to higher temperature when compared to the rubber without any compatibilizer. The use of an ENR/silane combination increases the tan $\delta$ at $5^{\circ} \mathrm{C}$ and reduces the $\tan \delta$ at $60^{\circ} \mathrm{C}$ compared to the use of ENR-51 alone, indicating an improvement in wet traction and rolling resistance of tires. However, overall the use of TESPT alone at optimum loading gives the best mechanical and dynamic mechanical properties compared to the presence of ENR as compatibilizer. The tensile rupture surfaces, as observed by SEM, of the silica-filled NR vulcanizates that are compatibilized using different compatibilizers correspond to their tensile strength, that is the materials of higher tensile strength show more tear lines and fracture surface roughness. 
Among the three types of silanes studied in combination with ENR-51 and sulfur compensation, the use of 2.2 phr TESPT together with 7.5 phr ENR-51 provides the best overall properties, but it does not fully match the properties of a compound with TESPT alone at optimal quantity, i.e. 4.7 phr. However, in the perspective of the "Magic Triangle of Tire Technology" where wet skid resistance, rolling resistance and wear are to be optimized, the combination of ENR-51, TESPT and sulfur compensation may become a better option for "Winter Tires" that demand good wet grip, providing that the real tire test has yet to be confirmed.

\subsection{References}

1. W.H. Waddell, L.R. Evans, Rubber Chem. Technol., 69, 377 (1996).

2. A. Voet, J.C. Morawski, J.B. Donnet, Rubber Chem. Technol., 50, 342 (1977).

3. S. Wolff, Rubber Chem. Technol., 69, 325 (1996).

4. I.R. Gelling, Rubber Chem. Technol., 58, 86 (1985).

5. A. Bandyopadhyay, M.D. Sarkar, A.K. Bhowmick, Rubber Chem. Technol., 77, 830 (2004).

6. C.S.L. Baker, I.R. Gelling, R. Newell, Rubber Chem. Technol., 58, 67 (1985).

7. A.K. Manna, P.P. De, D.K. Tripathy, S.K. De, D.G. Peiffer, J. Appl. Polym. Sci., 74, 389 (1999).

8. T.L.A.C. Rocha, C. Rosca, J. Ziegler, R.H. Schuster, Kautsch. Gummi Kunstst., 58, 22 (2005).

9. M.-J. Wang, S. Wolff, Rubber Chem. Technol., 65, 715 (1992).

10. J.W. ten Brinke, S.C. Debnath, L.A.E.M. Reuvekamp, J.W.M. Noordermeer, Comp. Sci. Technol., 63, 1165 (2003).

11. B.T. Poh, C.C. NG, Eur. Polym. J., 34, 975 (1998).

12. H. Yan, G. Tian, K. Sun, Y. Zhang, Y. Zhang, J. Appl. Polym Sci., 43, 573 (2005).

13. H. Yan, K. Sun, Y. Zhang, Y. Zhang, Y. Fan, J. Appl. Polym. Sci., 94, 1511 (2004).

14. H. Yan, K. Sun, Y. Zhang, Y. Zhang, J. Appl. Polym. Sci., 94, 2295 (2004).

15. J.L. Valentín, I. Mora-Barrantes, A. Rodríguez, L. Ibarra, L. Gonzalez, J. Appl. Polym. Sci., 103, 1806 (2007).

16 O. Klockmann, the International Tire Exbibition \& Conference (ITEC) 2006, September 12-14, 2006, Akron, OH, paper no.29B.

17. A. Blume, Kautsch. Gummi Kunstst., 64, 38 (2011).

18. W. Kaewsakul, K. Sahakaro, W.K. Dierkes, J.W.M. Noordermeer, Rubber Chem. Technol., 85, 277 (2012). 
19. G.G.A. Böhm, M.N. Nguyen, J. Appl. Polym. Sci., 55, 1041 (1995).

20. C.J. Lin, W.L. Hergenrother, E. Alexanian, G.G.A. Böhm, Rubber Chem. Technol., 75, 865 (2002).

21. S. Mihara, R.N. Datta, J.W.M. Noordermeer, Rubber Chem. Technol., 82, 524 (2009).

22. W. Salgueiro, A. Marzocca, A. Somoza, G. Consolati, S. Cerveny, F. Quasso, S. Goyanes, Polymer, 45, 6037 (2004).

23. H.M. Da Costa, L.L.Y. Viconte, R.C.R. Nunes, C.R.G. Furtado, J. Appl. Polym. Sci., 83, 2331 (2002).

24. L. Qu, G. Yu, L. Wang, C. Li, Q. Zhao, J. Li., J. Appl. Polym. Sci., 126, 116 (2012).

25. A.J. Manna, P.P. De, D.K. Tripathy, J. Appl. Polym. Sci., 84, 2171 (2002).

26. P. Saramolee, K. Sahakaro, N. Lopattananon, W.K. Dierkes, J.W.M. Noordermeer, Rubber Chem. Technol., 87, 320 (2014).

27. UNECE Regulation No 117, GRRF 71 ${ }^{\text {st }}$ session, September 13-15, 2011.

28. W. Arayapranee, N. Na-Ranong, G.L. Rempel, J. Appl. Polym. Sci., 98, 34 (2005). 


\section{Influence of Types of Silane Coupling Agents on the Reinforcement of Silica in Natural Rubber Compounds}

In this chapter, the effect of different types of silane coupling agents on the properties of silica-filled natural rubber (NR) compounds is studied in order to gain basic information for selecting a silane to be used later for preparation of silane-grafted NR. Three silane types: 3-Octanoylthio-1-propyltriethoxysilane (NXT), 3-mercaptopropyldi(tridecan-1-oxy-13-penta(ethyleneoxide))ethoxysilane (VP Si-363) and vinyltriethoxysilane (VTES) are used in the silica-filled NR compounds, based on molar and alkoxy functional group equivalents in comparison with conventional bis(triethoxysilylpropyl)tetrasulfide (TESPT). The addition of silane coupling agents in the silica-filled NR compounds reduces mixing energy, Mooney viscosity and Payne effect, while it improves the chemically bound rubber content and tensile properties. Increase of the silane coupling agent content to the same level of alkoxy functional groups as that of TESPT, results in improvement of both the compound and vulcanizate properties. Among the three types of silane, the use of VTES in silica-filled NR gives the poorest properties. With equivalent amounts of ethoxy groups, the use of NXT and reference TESPT results in the same level of tensile strength. The silica-filled NR compounds with NXT, VP Si-363 and VTES as coupling agents show inferior wet grip and rolling resistance, as far as can be derived from a lower tan $\delta$ at $5^{\circ} \mathrm{C}$ and higher tan $\delta$ at $60^{\circ} \mathrm{C}$, when compared to the reference compound with TESPT. With respect to the tire performance, NXT offers still better properties than VP Si-363 and VTES, respectively. 


\subsection{Introduction}

Based on the results in Chapters 3-5, even though epoxidized natural rubber (ENR) could promote filler-rubber interactions and improve both compound and vulcanizate properties to some extent, the presence of ENR as compatibilizer has a negative effect on the dynamic mechanical properties, especially with respect to tire rolling resistance. The combination of ENR and a small amount of silane coupling agent enhances the properties but the results remain inferior to the use of a silica/TESPT system in which the rubber matrix is homogeneous in absence of ENR. With the objective to modify NR by means of grafting silanes onto the NR molecules, in order to overcome the problems raised by the NR/ENR blend, several potential silanes are selected for this study. NXT with shielded mercapto function and VP Si-363 with a sterically hindered mercapto functional group, and VTES with a vinyl function are of interest, all commercially available. The chemical structures of the silanes are given in Table 6.1. Prior to performing the grafting reaction, the influence of these silanes on the properties of silicafilled NR compounds is investigated and reported in this chapter, in comparison with the use of TESPT as reference. This in order to know the reinforcing efficiency of silica in NR compounds when these silanes are applied as coupling agents.

Bis-(triethoxysilylpropyl)tetrasulfide (TESPT) has become the state-of-the-art in silica-silane technology and is most widely used in silica-filled rubber compounds. The silanization reaction of the ethoxy-groups in TESPT molecules with silanol groups of silica during mixing, and the coupling reaction between silane and rubber molecules during the vulcanization reaction lead to generation of chemical bonds between silica and rubber ${ }^{[1,2]}$, introducing a double network structure in the filled rubber; i.e. a network between filler and rubber via silane bridges and a rubber network generated by the curatives. The effective use of TESPT provides a good silica dispersion, low Payne effect, good abrasion resistance and mechanical properties, and low tire rolling resistance. ${ }^{[2-4]}$ TESPT itself has some drawbacks however, as the tetrasulfide structure can lead to "scorch" problems and the silanization reaction emits ethanol during mixing. Alternative silanes such as 3octanoylthio-1-propyltriethoxysilane (NXT) and 3-mercaptopropyl-di(tridecan-1-oxy-13penta(ethyleneoxide))ethoxysilane (VP Si-363) have been introduced, as previously mentioned in Chapter 5. NXT is an octanoyl-blocked mercaptosilane developed to provide a better scorch safety than TESPT for the silica-filled rubber compounds. ${ }^{[5]}$ However, the apparent activation energy of the vulcanization reaction of the NXT compound is lower than that of the TESPT compound, and the apparent activation energy of both compounds decreases with increasing silane concentration. ${ }^{[6]}$ VP Si-363 or 3-mercaptopropyl- 
di(tridecan-1-oxy-13-penta(ethyleneoxide))ethoxysilane is a mercaptosilane containing one ethoxy-group and two long alkoxy-groups. It has been reported that the presence of the thiol-group or the long alkoxy groups in the VP Si-363 structure significantly increases the reaction rate between silica and silane. Furthermore, the long alkoxy chains with oxygen atoms in the structure can increase the possibility of adsorption of this silane on the silica leading to a faster silane-silica reaction when compared to the mercaptosilane with short chain alkoxy groups. ${ }^{[7]}$ Compared with the use of TESPT, the incorporation of VP Si-363 into silica-filled styrene-butadiene rubber (SBR)/butadiene rubber (BR) blends for tire compounds improved the rolling resistance of a tire by $13 \%$ and reduced volatile organic compounds (VOC) emission by $80 \% .{ }^{[8,9]}$ A study on nanoclay-filled rubber showed that VP Si-363 has a smaller effect on tensile modulus than TESPT, which was possibly due to the bulky alkoxy group of VP Si-363 that took some space when bonded to the clay surface and restricted the number of bonds between silane and filler. ${ }^{[10]}$

In addition to the mercapto-silanes, there are still other types of silane coupling agents that have been tested for silica-filled polymers, and also for some other purposes, to include amino-silane, cyano-silane, vinyl silane, etc. Vinyl silanes such as vinyltrimethoxysilane (VTMS) and vinyltriethoxysilane (VTES) are applied for moisture crosslinking of polyolefins in order to improve their properties and expand the applications of these materials. ${ }^{[11-14]}$ In the rubber industry, vinyl silanes are commonly used to improve the properties of inorganic fillers-filled ethylene-propylene rubber (EPM) and ethylenepropylene-diene rubber (EPDM) with a peroxide vulcanization system. Vinyl-silane containing formulations show a remarkable improvement in modulus, tensile and tear properties, filler dispersion and dynamic mechanical properties of these materials. ${ }^{[15,16]}$ Besides a good interaction between filler and rubber as a result of the silanization reaction, the vinyl-group in the silane structure can also react with free radicals on rubber molecules during peroxide vulcanization ${ }^{[16]}$ leading to a strong bonding between filler and rubber, as reflected in good static and dynamic properties of the filled rubber. The presence of a $\mathrm{C}=\mathrm{C}$ structure in the vinyl silane offers the possibility for a grafting reaction of this silane onto the polymer chains if radicals are generated in the process.

In this chapter, the effect of different types of silane coupling agents: NXT, VP Si-363 and VTES, on the properties of silica-filled NR is studied by applying two sets of compounds based on equal molar and on alkoxy-functional groups relative to the reference compound with TESPT. The reinforcing efficiency of silica in combination with the different silanes in NR compounds is compared. Herein, the three types of silanes are studied with respect to their potential to be later used for preparation of silane grafted NR. 


\subsection{Experimental}

\subsubsection{Materials}

The compounding ingredients: natural rubber (Ribbed Smoked Sheet (RSS) \#3), highly dispersible silica (Zeosil 1165MP), treated distillate aromatic extract oil (TDAE-oil), $\mathrm{N}$-cyclohexyl-2-benzothiazole sulfenamide (CBS), diphenyl guanidine (DPG) and 2,2,4trimethyl-1,2-dihydroquinoline (TMQ), ZnO, stearic acid and sulfur, are as detailed in Chapter 3. Bis-(triethoxysilylpropyl)tetrasulfide (TESPT), 3-Octanoylthio-1propyltriethoxysilane (NXT) and 3-Mercaptopropyl-di(tridecan-1-oxy-13penta(ethyleneoxide))ethoxysilane (VP Si-363) are described with their producers in Chapter 5. Vinyltriethoxysilane (VTES) was supplied by Evonik (Germany). All ingredients were used as received. The chemical names, structures and molecular weights of all the silanes used in this chapter are shown in Table 6.1.

Table 6.1 Silane coupling agents information.

\begin{tabular}{|c|c|c|c|}
\hline $\begin{array}{l}\text { Silane } \\
\text { types }\end{array}$ & Chemical names & Structures & $\begin{array}{c}\text { MW } \\
(\mathrm{g} / \mathrm{mol})\end{array}$ \\
\hline TESPT & $\begin{array}{l}\text { Bis-(triethoxysilylpropyl) } \\
\text { tetrasulfide }\end{array}$ & 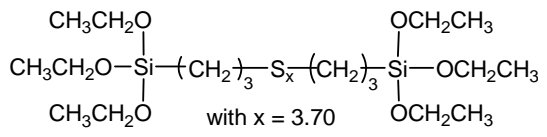 & 532.5 \\
\hline $\begin{array}{l}\text { VP Si- } \\
363\end{array}$ & $\begin{array}{l}\text { 3-Mercaptopropyl- } \\
\text { di(tridecan-1-oxy-13-penta } \\
\text { (ethyleneoxide))ethoxysilane }\end{array}$ & $\begin{array}{l}\mathrm{CH}_{3}\left(\mathrm{CH}_{2}\right)_{12}\left(\mathrm{OC}_{2} \mathrm{H}_{4}\right)_{5} \mathrm{O} \\
\left.\quad \mathrm{CH}_{3} \mathrm{CH}_{2} \mathrm{O}-\mathrm{Si}-\mathrm{CH}_{2}\right)_{3} \mathrm{SH} \\
\mathrm{CH}_{3}\left(\mathrm{CH}_{2}\right)_{12}\left(\mathrm{OC}_{2} \mathrm{H}_{4}\right)_{5} \mathrm{O}\end{array}$ & 987.5 \\
\hline NXT & $\begin{array}{l}\text { 3-Octanoyl-thio- } \\
\text { propyltriethoxysilane }\end{array}$ & 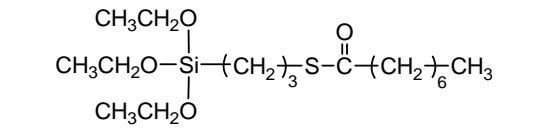 & 365 \\
\hline VTES & Vinyltriethoxysilane & $\begin{array}{l}\stackrel{\mathrm{CH}_{3} \mathrm{CH}_{2} \mathrm{O}}{\mathrm{CH}_{3} \mathrm{CH}_{2} \mathrm{O}-\mathrm{Si}-\mathrm{CH}=}=\mathrm{CH}_{2} \\
\mathrm{CH}_{3} \mathrm{CH}_{2} \mathrm{O}\end{array}$ & 190 \\
\hline
\end{tabular}

\subsubsection{Compound formulations and mixing procedures}

Compound formulations are shown in Table 6.2 and the amounts of NXT, VP Si363 and VTES silanes were calculated to either molar or alkoxy groups equivalents to that of the reference compound with TESPT. All the compounds were mixed following the mixing procedures as previously described in Table 5.3, but in the present case, only NR 
was used without ENR. The mixer and mixing conditions are as described in Section 5.2.2.

Table 6.2 Compound formulations.

\begin{tabular}{|c|c|c|c|c|c|c|c|c|}
\hline \multirow{3}{*}{$\begin{array}{l}\text { Ingredients } \\
\text { RSS3 }\end{array}$} & \multicolumn{8}{|c|}{ Parts per hundred parts of rubber (phr) } \\
\hline & \multicolumn{2}{|c|}{ References } & \multicolumn{3}{|c|}{ Molar equivalents } & \multicolumn{3}{|c|}{$\begin{array}{l}\text { Alkoxy groups } \\
\text { equivalents }\end{array}$} \\
\hline & 100.0 & 100.0 & 100.0 & $\begin{array}{c}100 . \\
0\end{array}$ & 100.0 & 100.0 & 100.0 & $\begin{array}{c}100 . \\
0\end{array}$ \\
\hline TESPT & - & $4.7^{*}$ & - & - & - & - & - & - \\
\hline NXT & - & - & 3.2 & - & - & 6.4 & - & - \\
\hline VP Si-363 & - & - & - & 8.7 & - & - & 17.4 & - \\
\hline VTES & - & - & - & - & 1.7 & - & - & 3.4 \\
\hline $\begin{array}{l}\text { Zeosil } \\
1165 \mathrm{MP}\end{array}$ & 55.0 & 55.0 & 55.0 & 55.0 & 55.0 & 55.0 & 55.0 & 55.0 \\
\hline TDAE oil & 8.0 & 8.0 & 8.0 & 8.0 & 8.0 & 8.0 & 8.0 & 8.0 \\
\hline $\mathrm{ZnO}$ & 3.0 & 3.0 & 3.0 & 3.0 & 3.0 & 3.0 & 3.0 & 3.0 \\
\hline TMQ & 1.0 & 1.0 & 1.0 & 1.0 & 1.0 & 1.0 & 1.0 & 1.0 \\
\hline Stearic acid & 1.0 & 1.0 & 1.0 & 1.0 & 1.0 & 1.0 & 1.0 & 1.0 \\
\hline DPG & 1.0 & 1.0 & 1.0 & 1.0 & 1.0 & 1.0 & 1.0 & 1.0 \\
\hline CBS & 1.5 & 1.5 & 1.5 & 1.5 & 1.5 & 1.5 & 1.5 & 1.5 \\
\hline Sulfur & 1.5 & 1.5 & 1.5 & 1.5 & 1.5 & 1.5 & 1.5 & 1.5 \\
\hline
\end{tabular}

Remarks: * TESPT $4.7 \mathrm{phr}$ equals $8.8 \mathrm{mmol}$.

\subsubsection{Testing of compound and vulcanizate properties}

Mooney viscosity, Payne effect, bound rubber content and cure characteristics of the compounds were tested using the methods and equipments as described in Chapter 5. The compounds were cured to their respective optimum cure times, and the resulting vulcanizates were tested for their tensile and dynamic mechanical properties, also as described in Chapter 5.

\subsection{Results and discussion}

\subsubsection{Mixing behaviors}

The processability of the rubber compounds can initially be determined by their mixing behavior and the torques generated during mixing, which are associated with the compound viscosities. The plots of mixing torques versus mixing time of the compounds with equal molar and alkoxy-functional groups of NXT, VP Si-363 and VTES compared to TESPT respectively, are shown in Figures 6.1-6.3. The mixing torque of the filled compounds largely depends on the level of filler dispersion. By using a bifunctional silane 
with alkoxy groups, the silanization reaction between the alkoxy groups of the silane and the silanol groups of the silica takes place in the mixer. A TESPT-molecule contains an average 3.7 sulfur atoms and two sides of triethoxy groups that can effectively react with the silanol groups on the silica surface under optimum mixing conditions. It has been reported that the premature crosslinks created by the sulfur in TESPT in silica-filled NR compounds showed a positive effect on silica dispersion as it helped to suppress filler flocculation. ${ }^{[17]}$ The NXT, VP Si-363 and VTES are also bifunctional silanes with different chemical structures, which may have an influence on the reactivity towards the silanization and later coupling reaction with the rubber.

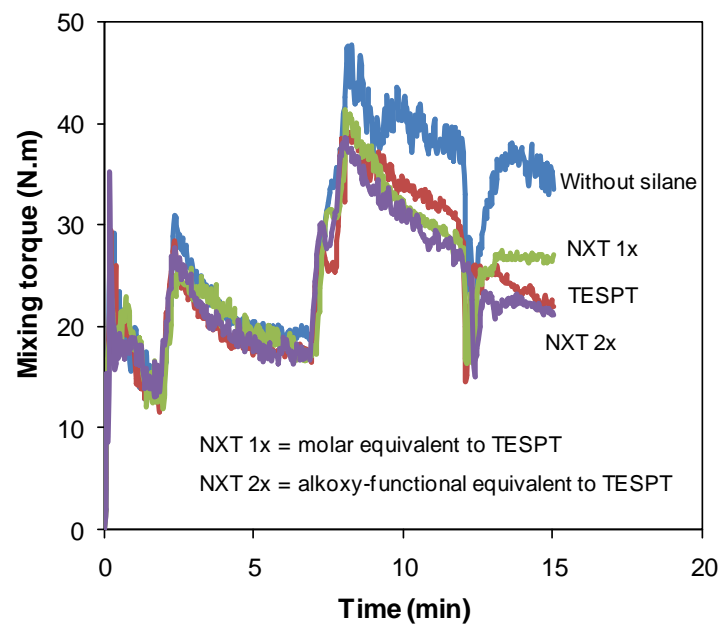

Figure 6.1 Mixing torques of silica-filled NR with NXT in comparison with TESPT and without silane coupling agent.

Figure 6.1 shows the mixing torques of silica-filled NR compounds without silane coupling agent, with TESPT, and with NXT at two different contents based on equal molar and ethoxy-groups relative to TESPT. As expected, the silica-filled NR compound without silane coupling agent clearly shows the highest mixing torque, related to the highest compound viscosity due to strong filler-filler interaction that causes formation of a fillerfiller network of silica aggregates and restricts the mobility of the rubber. Moreover, some parts of rubber are trapped inside the silica network, i.e. occluded rubber. It has been demonstrated that the silica-silica network and a high amount of occluded rubber contributes to the shear modulus of the uncured compounds ${ }^{[18]}$, that shall reflect in the mixing torque. The incorporation of silane coupling agents in the silica-filled compounds 
clearly improves the compound processability as seen in the reduction of mixing torques especially after the addition of the $2^{\text {nd }}$ half of the filler plus silane and oil, as a result of improved silica dispersion and presence of the process oil. At the beginning, the silane addition is comparable to a plasticizer: it reduces the viscosity. At the end of the mixing cycle, the compound with NXT at equal ethoxy-functional groups to TESPT shows a slightly lower mixing torque compared to the reference compound with TESPT.

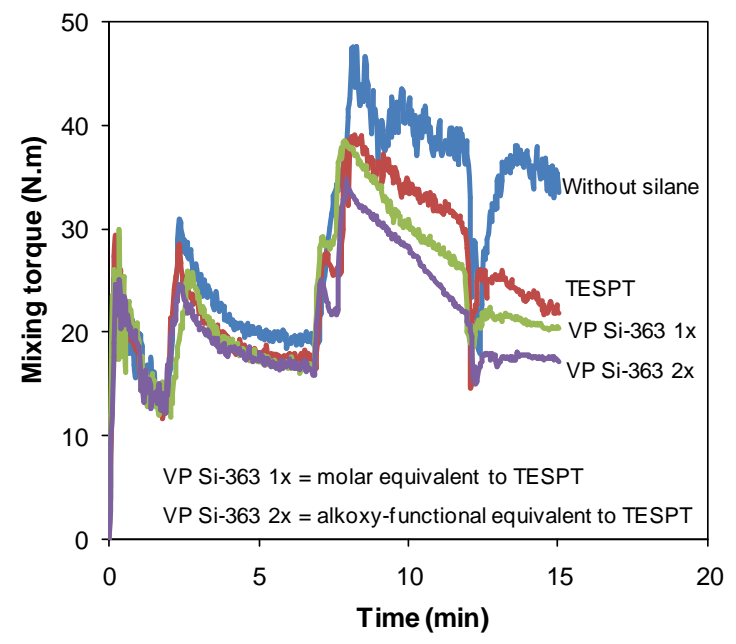

Figure 6.2 Mixing torques of silica-filled NR with VP Si-363 in comparison with TESPT and without silane coupling agent.

The use of VP Si-363 at both loadings in the silica-filled NR compounds results in a remarkably lower mixing torque than the compound with TESPT as seen in Figure 6.2. The mixing torque decreases with increasing amount of VP Si-363 and the difference in mixing torques can be clearly observed in the second interval of silica addition already, indicating a very fast dispersion of silica in the rubber matrix. As reported by Blume ${ }^{[7]}$, the presence of the -SH and two long alkoxy groups in VP Si-363 increases the rate of reaction with the silica due to the increase of adsorption possibilities to the silanol-groups which can occur via the ethoxy-group, thiol and different oxygen atoms inside the alkoxy chain through hydrogen bonding, as proposed in Scheme 6.1. Besides the reaction between the ethoxy-group and the silanol-group, the long alkoxy chains that shield the silica surface promote the silica dispersion and improve the processability of the silicafilled rubber compound. 


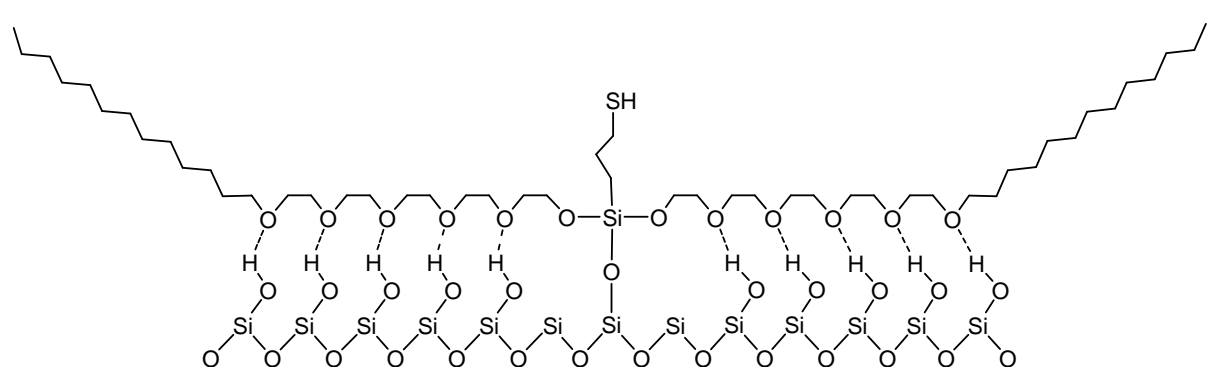

Scheme 6.1 Proposed interaction mechanism between VP Si-363 silane and silanol groups on the silica surface via hydrogen bonds. ${ }^{[7]}$

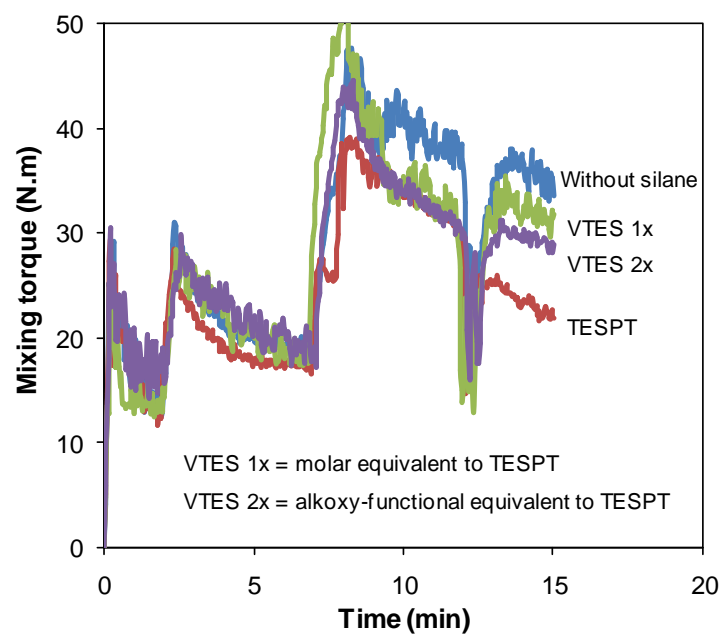

Figure 6.3 Mixing torques of silica-filled NR with VTES in comparison with TESPT and without silane coupling agent.

In contrast to the other two silanes, the addition of vinyl-silane VTES results in final mixing torques which are higher than for the compound containing TESPT, but still lower than without silane, as shown in Figure 6.3. At equal amount of ethoxy-functional groups of TESPT and VTES, the VTES-containing compound still shows a higher mixing torque compared to the use of TESPT. As the silane molecule reacts with the silica via the ethoxy groups and the chemical structure of the whole silane influences its kinetic behavior ${ }^{[7]}$, the VTES with the short vinyl function that gives less steric hindrance compared to NXT and VP Si-363, should provide a better reactivity towards the silanol groups. According to the study by Blume ${ }^{[7]}$ with a model compound, the rate of the beginning reaction of VTES is slightly higher than for VP Si-363 and clearly higher than for 
TESPT. Despite the higher rate of the beginning reaction of VTES over TESPT, the compound with VTES shows a higher mixing torque, i.e. higher viscosity. The smaller steric effect of VTES molecules leads to a lesser shielding effect on the silica surface. Moreover, there may be a loss of some ethoxy-groups of VTES through the hydrolysisreaction with moisture and a consequent condensation reaction with neighboring ethoxygroups due to their high reactivity. For the case of TESPT, the bulky molecule introduces steric hindrance and reduces the rate of condensation between its own molecules, and in addition TESPT has a larger shielding effect on the silica surface compared to VTES, resulting in the possibly better silanization efficiency and filler dispersion.

Table 6.3 Characteristics of mixing behavior of silica-filled NR compounds with different silane types.

\begin{tabular}{lccc}
\hline \multicolumn{1}{c}{ Compounds } & $\begin{array}{c}\text { Silane content } \\
(\mathbf{m m o l})\end{array}$ & $\begin{array}{c}\text { Specific energy } \\
(\mathbf{k J} / \mathbf{g})\end{array}$ & $\begin{array}{c}\text { Dump temperature } \\
\left({ }^{\circ} \mathbf{C}\right)\end{array}$ \\
\hline Without silane & - & 4.37 & 156 \\
TESPT & 8.8 & 3.43 & 144 \\
\hline NXT 1x & 8.8 & 3.48 & 144 \\
VP Si-363 1x & 8.8 & 3.13 & 138 \\
VTES 1x & 8.8 & 3.89 & 148 \\
\hline NXT 2x & 17.6 & 3.32 & 142 \\
VP Si-363 2x & 17.6 & 2.94 & 134 \\
VTES 2x & 17.6 & 3.80 & 146 \\
\hline
\end{tabular}

The incorporation of rigid filler particles into rubber compounds increases the viscosity and impedes the flow of rubber molecules, causing a higher mixing energy and an increase of compound temperature due to generated heat. The silanization reaction between the alkoxy-groups of the silanes and the polar silanol groups of silica leads to less filler-filler interaction and improvement of silica dispersion in the rubber matrix, and consequently affects compound viscosity and mixing torque. ${ }^{[3]}$ Based on the mixing torque data, the mixing energy $(\mathrm{kN} . \mathrm{m} / \mathrm{kg})$ was obtained and the specific energy in units of $(\mathrm{kJ} / \mathrm{g})$ for each compound was calculated, and the values summarized in Table 6.3. As already observed in the mixing torque curves in Figures 6.1-6.3, the use of silane coupling agents reduces the mixing torques and the specific energy is in accordance with the mixing torques or compound viscosity. Lower viscosity generates less shearing heat during mixing and results in a lower dump temperature, as also shown in Table 6.3. Among the four types of silanes, VP Si-363 gives the lowest specific mixing energies and dump 
temperatures whereas VTES shows the highest values. The use of higher silane contents in the compounds in case of functional groups-equivalence to TESPT leads to less mixing energy and lower dump temperature. In addition to the improvement of silica dispersion and filler-rubber interactions, a silane itself can also act as a plasticizer that helps the mobility of the rubber chains and therefore reduces viscosity.

\subsubsection{Mooney viscosity, Payne effect and bound rubber content}

The properties of filled uncured compounds generally correspond to the level of filler dispersion, filler-rubber interactions and the occurrence of a filler-filler network. Mixing of silica into NR or other hydrocarbon rubbers is known to face difficulties arising from the strong silica-silica interactions and poor silica-rubber compatibility. Different compatibilizers or silane coupling agents will have a different efficiency in silica-filled NR compounds which affects the properties of the compounds. The use of NXT, VP Si-363 and VTES in comparison with TESPT affects Mooney viscosity, Payne effect and bound rubber content of the compounds as follows.

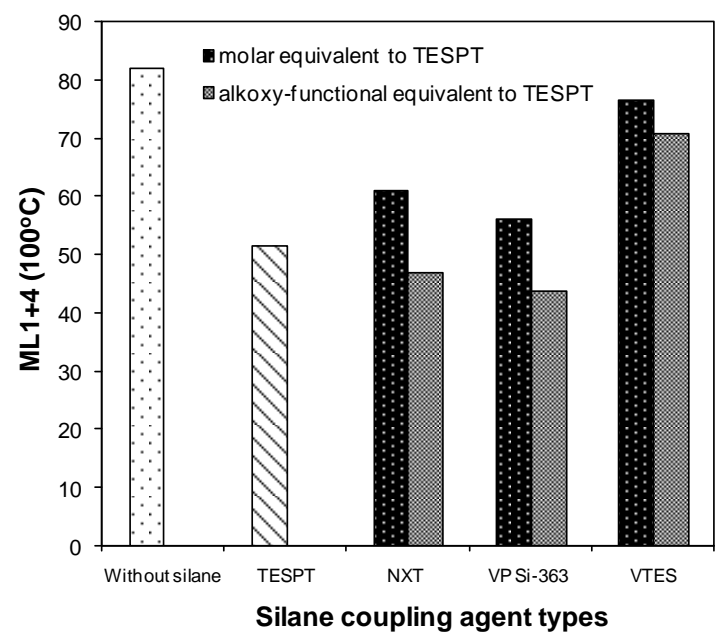

Figure 6.4 Mooney viscosity of silica-filled NR compounds with different types and amounts of silane coupling agents.

Mooney viscosities of the silica-filled NR compounds silanized with different types of silane coupling agent are shown in Figure 6.4. The silica-filled NR compound without silane shows a significantly higher Mooney viscosity than all other compounds in accordance with the mixing torque result, as discussed previously. At the same molar 
content, the compound with TESPT shows the lowest Mooney viscosity indicating its superior flow due to the good silica shielding by the six ethoxy-groups. Among the compounds with silanes, the use of VTES gives the highest compound Mooney viscosity, independent whether it was used at either molar or functional equivalents to TESPT. This poorest efficiency of VTES to enhance filler dispersion, by means of silanization and shielding off the silanol groups, despite its high reactivity due to less steric effects, may be attributed to the possibility of self-reaction between the VTES molecules as proposed in Scheme 6.2. At the high temperature during mixing, the reactive ethoxy groups of VTES may be hydrolyzed or directly condensed with neighboring ethoxy groups leaving less functional groups to react with the silanol moieties on the silica surface and a minor shielding-effect. Moreover, after the silanization of the silica the short vinyl-group on the other side of the VTES molecule cannot promote molecular chain movement like in the case of the long fragments of NXT and VP Si-363 molecules.<smiles>C=C[Si](OCC)(OCC)OCC</smiles><smiles>C=C[13CH2]C=C[Si](O)(O)OCC</smiles>

Scheme 6.2 Possible self-condensation reaction of VTES molecules.

At equal molar basis, the Mooney viscosity of the compound with TESPT is lower than with VP Si-363 and NXT, but at the equal functional groups content the compound with VP Si-363 shows the lowest Mooney viscosity of all. The addition of VP Si-363 results in a lower Mooney viscosity than the use of NXT, because besides the silanization reaction between the alkoxy groups of the silane and the silanol groups of silica, two long alkoxy groups with different oxygen atoms promote the adsorption of silane on silica via hydrogen bonding, as shown in Scheme $6.1{ }^{[7]}$ Furthermore, the efficient shielding of the silica surface and the long alkyl chains enhance the mobility of rubber chains around silica aggregates leading to the low compound viscosity. 

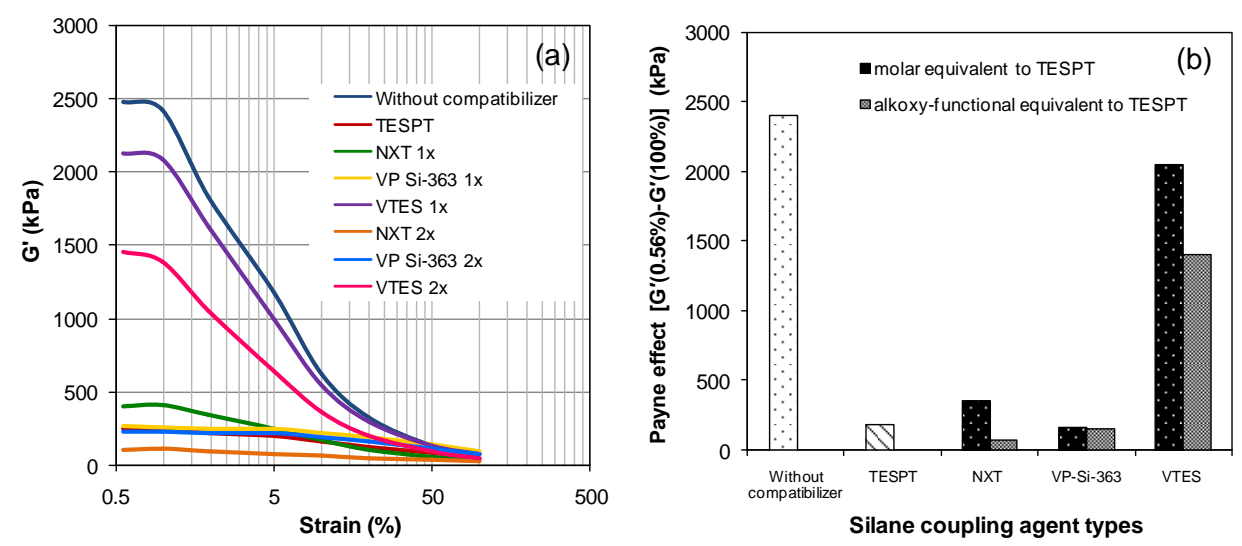

Figure 6.5 Storage modulus versus strain (a) and Payne effect [ $\left.G^{\prime}(0.56 \%)-G^{\prime}(100 \%)\right]$ (b) of silica-filled NR compounds with different types and amounts of silane coupling agents.

Figures 6.5 (a) and (b) show the Payne effect or filler-filler interaction of the silica-filled NR compounds. The plots of storage shear modulus $G^{\prime}$ versus strain amplitude in Figure 6.5 (a) clearly show a strong filler-filler interaction of the compound without silane and only a small reduction of the Payne effect by the use of VTES. Increasing the VTEScontent reduces the Payne effect, but it is still very much higher than the use of TESPT, VP Si-363 and NXT which can effectively diminish the filler-filler interactions in the compounds. This is clearly seen in Figure 6.7 (b) in which the difference of $\mathrm{G}^{\prime}$ at $0.56 \%$ and $100 \%$ strain is presented. The lower filler-filler interaction, i.e. less filler-filler networking and better dispersion of the silanized silica-filled compounds with TESPT, NXT and VP Si-363 is in good agreement with the compound viscosities: Figure 6.4, and mixing torques: Figures 6.1-6.3, as previously discussed. With the same alkoxy-group content, NXT gives even a lower Payne effect compared to TESPT. This difference may be caused by the smaller size and less steric hindrance of the NXT molecules compared to TESPT, which leads to an easier reaction between the alkoxy-group of NXT-silane and the silanol groups of silica. Another effect is that TESPT can couple two silica-aggregates which slightly increases the viscosity. 

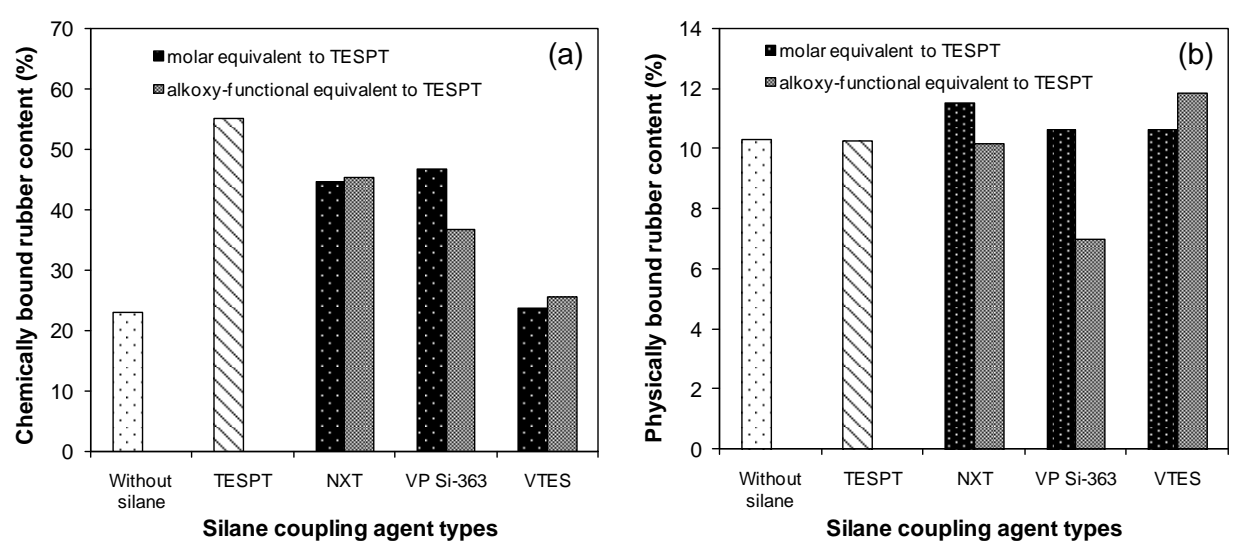

Figure 6.6 Chemically bound rubber (a) and physically bound rubber content (b) of silicafilled NR compounds with different types and amounts of silane coupling agents.

The interactions between silica and the rubber phase promoted by silane can be proved by the chemically bound rubber content ${ }^{[19]}$ in the silica-filled NR compounds as shown in Figure 6.6 (a). Among the various types of silane studied, the silica-filled NR compound with TESPT shows the highest chemically bound rubber content, whereas the compounds with NXT and VP Si-363 display similar values lower than for TESPT but still higher than for the mixes with VTES and without silane. All the compounds show similar physically bound rubber contents as shown in Figure 6.6 (b). The somewhat low value for VP Si-363 is probably due to the large measurement error for the low values of the physically bound rubber, being the difference of two large numbers: total-chemically bound rubber. The good filler-rubber interactions in the compounds with the sulfur containing silanes, i.e. TESPT, NXT and VP Si-363, is not only the result of an efficient silanization reaction but also because of an increase of the adsorption possibility on the silica surface by thiols. The exceptionally high chemically bound rubber content in the TESPT compound can be attributed to the tetrasulfide structure that can donate free sulfur into the system to generate some crosslinks in the compound, as previously demonstrated by Kaewsakul et al. ${ }^{[17]}$ The lowest chemically bound rubber of the compound with VTES confirms that it has the lowest efficiency due to its minor shielding effect for use with silica in NR-compounds compared to the other silane coupling agents. The decrease of the physically and chemically bound rubber contents in the VP Si-363 silanized silica-filled NR compounds when the amount of silane was increased could be due to its molecular structure that has a long alkoxy-group. The ethoxy-groups will react with silanol-groups of the silica, although the oligomeric poly(ethyleneoxide) side-arms will also react to some 
extent, in the same manner as poly(ethyleneoxide) itself can act as a shielding agent for silica. The two long alkoxy chains per molecule should also improve the rubber chain mobility around the silica aggregates and cause more loose chains to be dissolved in toluene during the bound rubber content measurement.

The different structures of silanes on the silica surface after silanization and the linkages between silane and rubber chains caused by either premature scorch in the TESPT compound or after vulcanization in the compounds with TESPT, NXT and VP Si363, are proposed in Scheme 6.3. 
Before vulcanization

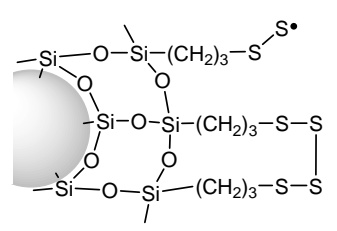

TESPT silanized silica

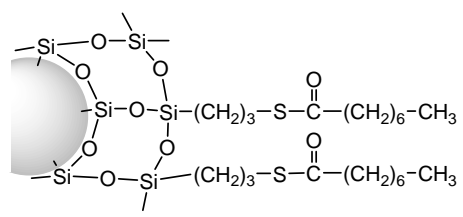

NXT silanized silica

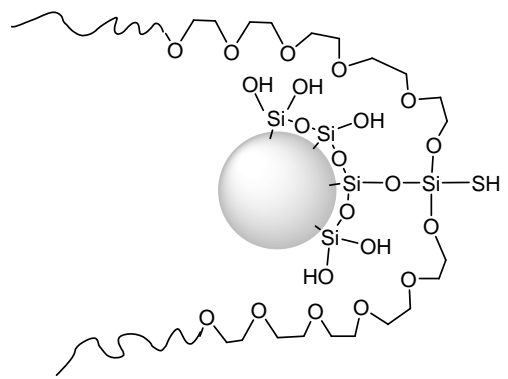

VP Si-363 silanized silica<smiles>C=C[Si]1(C)O[Si]2(C)O[Si](C)(C)O[Si]3(C=C)O[Si](C)(O2)O[Si](C=C)(O1)O3</smiles>

VTES silanized silica
After vulcanization
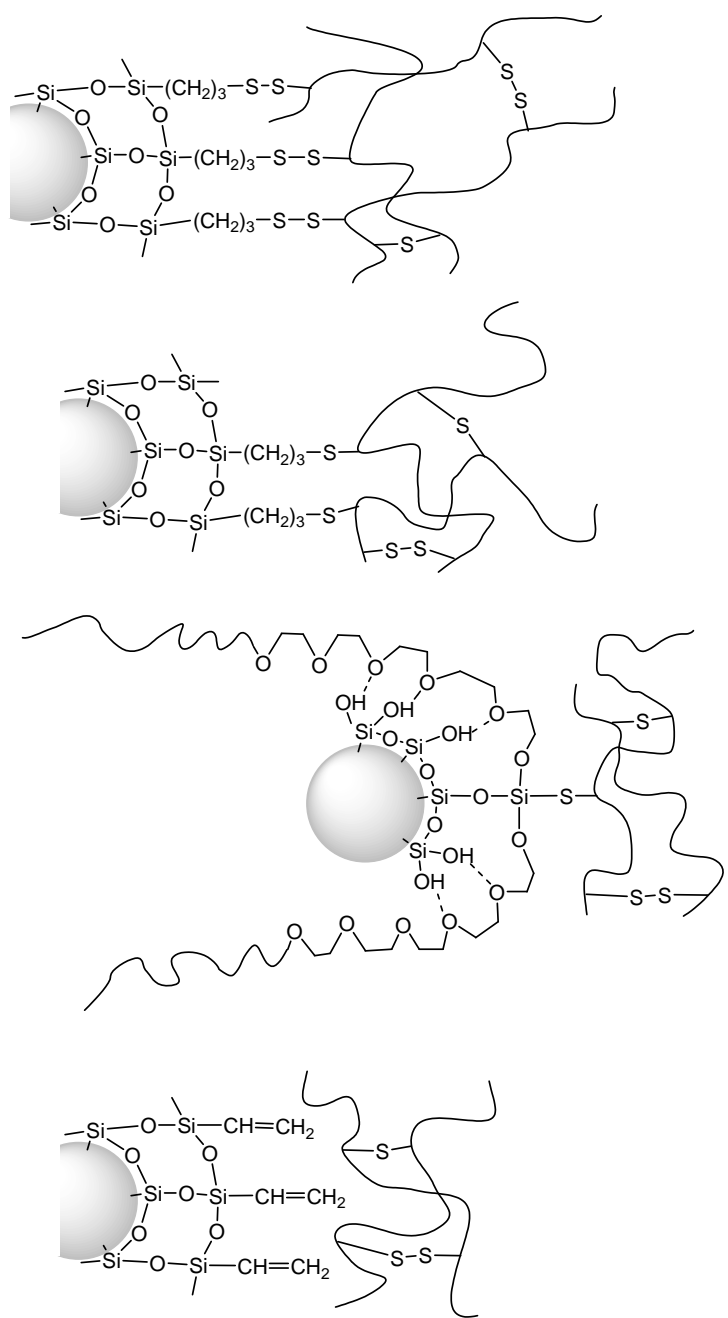

Scheme 6.3 Different structures of silanes on the silica surface after silanization and coupling, before and after vulcanization.*)

\footnotetext{
*) The lateral condensation of silanes attached to adjacent isolated silanol-sites as depicted in this Scheme 6.3 has often been quoted in literature. After the present project was completed it came to our attention, that based on molecular modeling for steric reasons this reaction can only take place for a minimum distance $>4 \AA$, which surpasses the distance between adjacent silanol-sites by a factor of appr. 2. (A.Blume, M. El-Roz, F.Thibault-Starzyk. Presented at KHK $11^{\text {th }}$ Fall Rubber Colloquim, Hanover, Germany, November 26-28, 2014).
} 


\subsubsection{Cure characteristics}
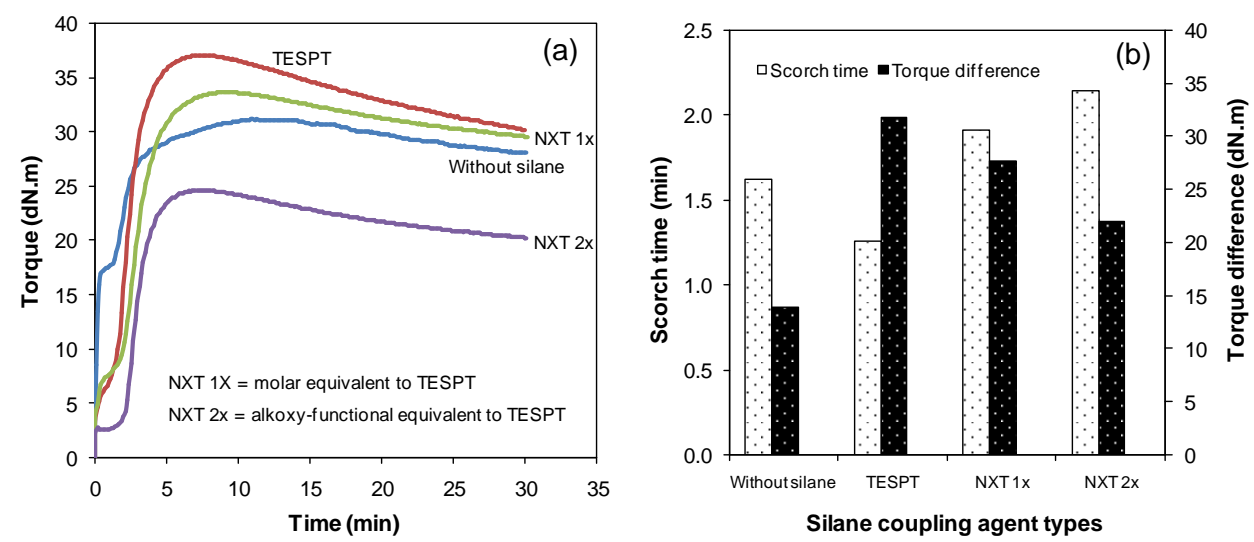

Figure 6.7 Cure curves (a) and scorch time and torque difference (b) of silica-filled NR compounds with NXT silane compared to the compounds with TESPT and without silane.

NXT silane is a mercapto silane with a carboxylic blocking group to retard the reactivity of the mercapto-group. Basically, the NXT-compound is more stable at high temperature than TESPT, providing a better scorch safety. As reported by Yan et al. ${ }^{[6]}$, the scorch time of a NR compound with NXT is longer than that with TESPT due to the bonding dissociation energy of NXT, which is higher than that of TESPT. However, the apparent activation energy of vulcanization of the compound with NXT is lower than that with TESPT at a given concentration due to the steric hindrance of NXT which is less than for TESPT. From the cure curves of silica-filled NR compounds in Figure 6.7 (a) the compound without silane coupling agent clearly shows silica flocculation which means reagglomeration of silica under shear conditions at high temperature. ${ }^{[20]}$ The addition of TESPT and NXT suppresses silica flocculation due to increased filler-rubber interaction through the silanization reaction and so decrease the filler-filler interaction. The use of NXT and TESPT silanes at the same molar content results in a similar rheometer minimum cure torque $\left(\mathrm{M}_{\mathrm{L}}\right)$ in which the NXT compound shows a little silica flocculation, but after vulcanization, the maximum cure torque $\left(M_{H}\right)$ and torque difference $\left(M_{H}-M_{L}\right)$ of the NXT compound is lower than that of the TESPT compound, as shown in Figure 6.7(b). Increase of the NXT silane loading in order to have the same amount of alkoxy-groups as TESPT leads to a significant decrease of $M_{L}$ without a sign of silica flocculation and longer induction period prior to vulcanization, as well as a lower $\mathrm{M}_{H}$ due to its plasticization- in combination with silanization-effects. The scorch time of the silica-filled NR compound 
without silane is longer than that of the compound with TESPT due to the polar character of the silica surface that adsorbs the polar curatives and results in cure retardation as well as less vulcanization efficiency. In contrast, the addition of NXT-silane gives a longer scorch time than the compounds without silane and with TESPT, respectively, due to the carboxylic blocking group in the NXT-structure that results in more thermal stability than the TESPT silane. The higher sulfur rank of TESPT with lower bonding energy of S-S in TESPT compared to the C-S in NXT also leads to a higher reactivity of TESPT towards coupling with rubber during the vulcanization reaction. TESPT may "donate" some of its sulfur to the compound, to implicitly raise the amount of free sulfur and consequently give extra crosslinking. NXT cannot do that.
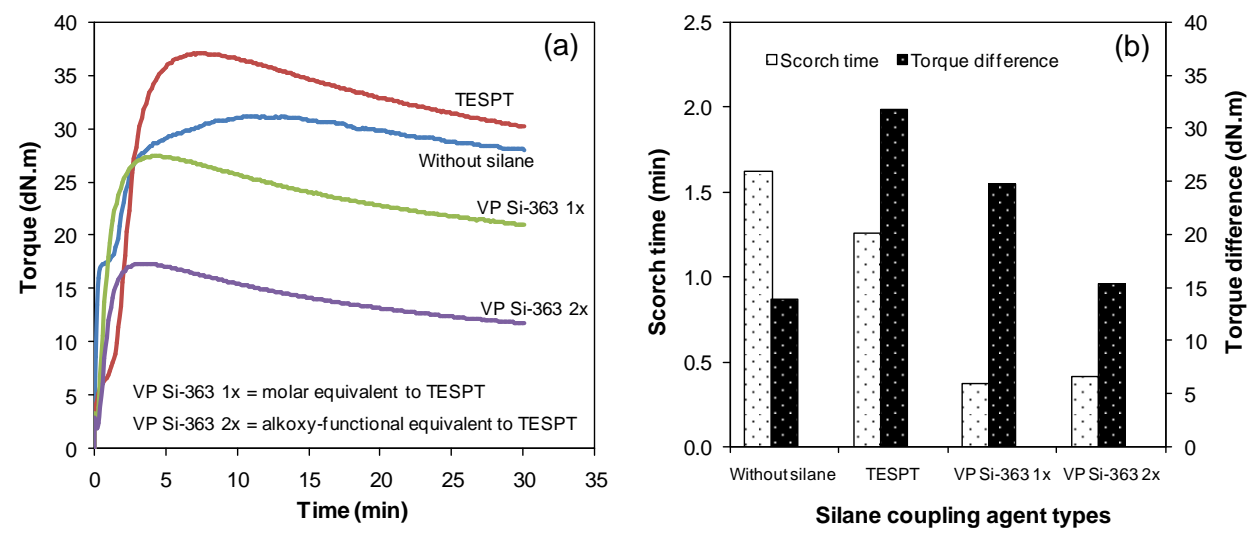

Figure 6.8 Cure curves (a) and scorch time and torque difference (b) of silica-filled NR compounds with VP Si-363 silane compared to the compounds with TESPT and without silane.

Figure 6.8 (a) shows the cure curves of silica-filled NR compounds with two different amounts of VP Si-363 in comparison with that of the compounds with TESPT and without silane. VP Si-363 is a mercapto-silane with one ethoxy-group and two bulky long alkoxy-groups. The reactive mercapto-group in the VP Si-363 molecule leads to a very fast curing reaction as seen by the shorter scorch and cure times compared to the compound with TESPT. The very short scorch time due to the highly reactive thiol endgroup of VP Si-363 can be clearly seen in Figure 6.8 (b). The compounds with VP Si-363 show a lower minimum torque than that with TESPT and no silica flocculation. Increasing the amount of VP Si-363 drastically decreases the cure torque maximum and torque difference: Figure 6.8 (b), which may be caused by a plasticizing effect of the long alkoxy- 
groups, leading to a reduction of stiffness of the final vulcanizate. Again, TESPT outperforms VP Si-363 in maximum torque due to its sulfur donating effect.
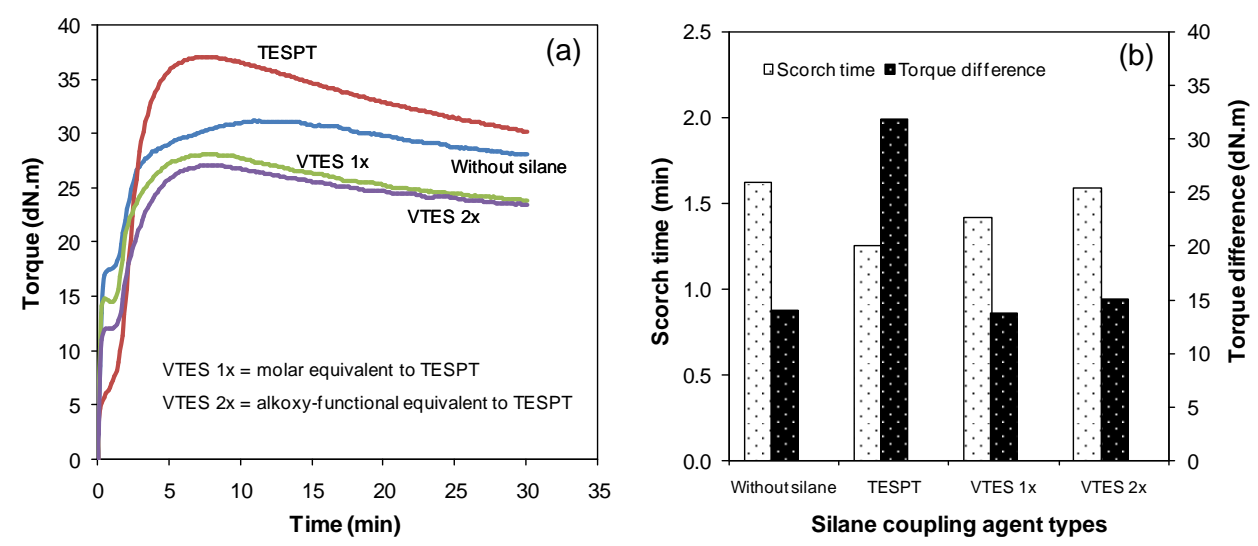

Figure 6.9 Cure curves (a) and scorch time and torque difference (b) of silica-filled NR compounds with VTES silane compared to the compounds with TESPT and without silane.

Among the four types of silanes used in this work, VTES is a sulfur-free silane with tri-ethoxy and vinyl-functional groups. The silica-filled NR compounds with VTES silane show a reduction of silica flocculation with increasing VTES content, but the flocculation phenomenon still clearly exists even in the compound with ethoxy-groups equivalent to TESPT in accordance with the high Payne effect: Figure 6.5, and low chemically bound rubber content: Figure 6.6 (a). As discussed previously, the small molecule of VTES can easily react with the silanol groups of silica but not properly shield the silica surface leading to a lower silanization efficiency between silica and silane. Furthermore, the vinyl-structure does not enhance the adsorption on the silica surface like with the other silanes. As observed in Figures 6.9 (a) and (b), increasing the amount of VTES has only a little effect on the cure behavior of the compounds. The absence of sulfur in the VTES/silica compound leads to a lack of coupling reactions via the sulfur atoms and consequently lower network density, resulting in a lower maximum cure torque and torque difference compared to the TESPT compound. The compound without silane has a filler-filler network that results in a higher maximum torque $\mathrm{M}_{\mathrm{H}}$ compared to the one with VTES, but due to its higher minimum torque $M_{L}$, the torque differences $\left(M_{H}-M_{L}\right)$ of the compounds without silane and with VTES are similar. During vulcanization via a radical 
mechanism, the vinyl-part may be bound to the rubber molecules, but this apparently did not happen to a significant extent as the sulfur vulcanization is not a radical reaction.

\subsubsection{Tensile and dynamic mechanical properties}
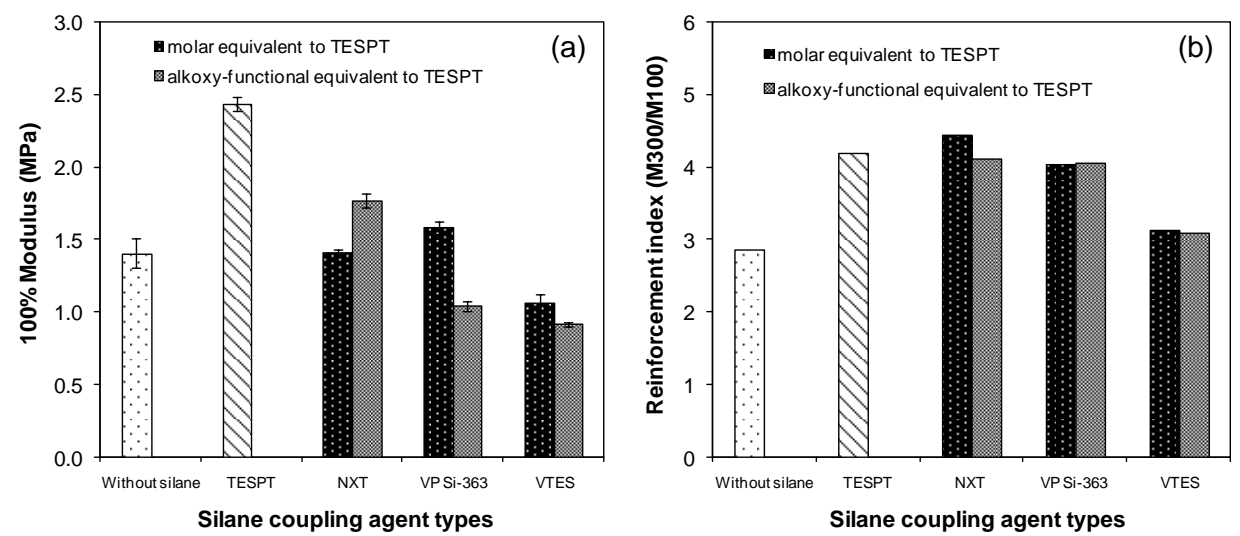

Figure 6.10 Modulus at $100 \%$ strain (a) and reinforcement index (b) of silica-filled NR compounds with different types and amounts of silane coupling agents compared to that of the compounds with TESPT and without silane.

Figure 6.10 shows the $100 \%$-modulus and reinforcement index of the silica-filled NR vulcanizates containing different types of silanes in which the one with TESPT shows the highest modulus, in correspondence with its highest chemically bound rubber: Figure 6.6, and maximum cure torques: Figures 6.7-6.9. Compared with NXT and VP Si-363 silanes, either at equal molar or number of alkoxy-groups, TESPT that consists of a higher sulfur rank gives a remarkably higher tensile modulus contributed by a higher crosslink density because of its higher sulfur content and sulfur donating ability. The lower bonding dissociation energy of TESPT compared to NXT and VP Si-363 leads to a faster and easier reaction with rubber. The bonding dissociation energy in sulfur-containing silanes is as follows: C-S-C $285 \mathrm{~kJ} / \mathrm{mol}$; C-S ${ }_{x}-\mathrm{C}$ (where $\mathrm{x} \geq 2$ ) $<268 \mathrm{~kJ} / \mathrm{mol}$; S-S $\approx 134 \mathrm{~kJ} / \mathrm{mol}^{[6,21]}$ Consequently, there are more chemical bonds between TESPT and rubber and a higher crosslink density because of the extra sulfur donated by TESPT leading to higher stiffness, i.e. higher modulus. At equal moles of silanes used, the vulcanizates with NXT and VP Si-363 silanes show significantly lower moduli compared to that with TESPT, but clearly higher than that with VTES. The differences in tensile modulus of the vulcanizates containing different silane types resemble the differences in chemically bound rubber content, as shown in Figure 6.6. The inferior modulus of the vulcanizate with VTES 
demonstrates that it suffers from absence of coupling reaction even though it shows some improvement in the properties of uncured compounds such as the Payne effect compared to the compound without silane.
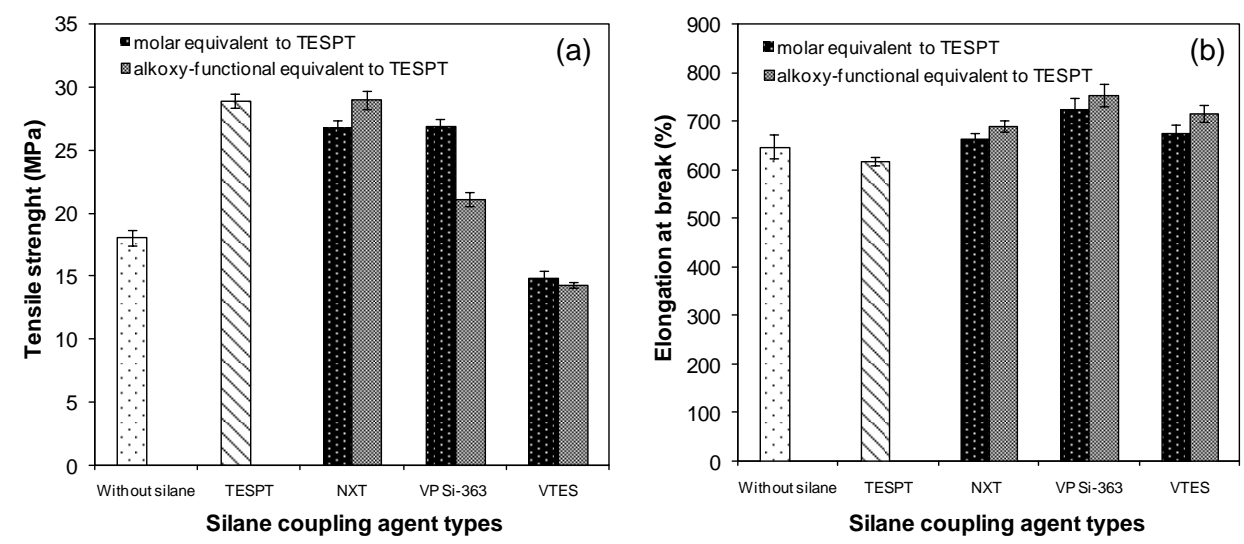

Figure 6.11 Tensile strength (a) and elongation at break (b) of silica-filled NR compounds with different types and amounts of silane coupling agents compared to that of the compounds with TESPT and without silane.

The tensile strength of the silica-filled NR vulcanizates having different types of silane coupling agents as shown in Figure 6.11(a) shows the same trend as that of the $100 \%$ modulus: Figure 6.10(a) is in accordance with the chemically bound rubber content: Figure 6.6(a). The state-of-the-art TESPT gives superior modulus and tensile strength compared to the other silanes. However, the addition of NXT silane at equal amount of alkoxy groups also results in a tensile strength at the same level as the use of TESPT. At the same molar equivalents to TESPT, NXT and VP Si-363 give vulcanizates with the same level of tensile strength, slightly lower than TESPT. But an increasing amount of VP Si-363 decreases the tensile strength like for the $100 \%$ modulus, while the use of VTES shows no improvement in tensile properties compared to the compound without silane. The elongation at break of the vulcanizates without and with different silanes show only small differences, where the vulcanizates with higher $100 \%$ modulus tend to have a lower elongation at break. The maximum extent of deformation also correlates with the maximum cure torque $\left(\mathrm{M}_{\mathrm{H}}\right)$ which may be taken as an indication for the crosslink or network density in the materials. As seen in Figure 6.8, the NR with VP Si-363 has a significantly lower $\mathrm{M}_{\mathrm{H}}$ compared to the vulcanizates without silane and with TESPT; it can sustain larger deformation before breaking. 
Tensile strength and modulus at $300 \%$ elongation show a slight correlation with the chemically bound rubber content as shown in Figures 6.12(a) and (b), respectively. Different types of silane show a different reinforcing efficiency depending on their functional groups and structure, that influence the level of interaction and/or reaction between silica and rubber. The use of TESPT, NXT and VP Si-363 significantly increases the chemically bound rubber content compared to the non-compatibilized compound, and consequently there is a large increase seen in tensile strength and $300 \%$-modulus. The use of TESPT gives the highest chemically bound rubber content and the highest tensile strength and $300 \%$-modulus of the silica-filled NR vulcanizates. The addition of VTES causes no change in the chemically bound rubber content and even slightly lowers the tensile strength and $300 \%$-modulus compared to the compound without silane. It is worth to note that the use of NXT and VP Si-363 gives comparable tensile strengths but lower moduli compared to the use of TESPT.
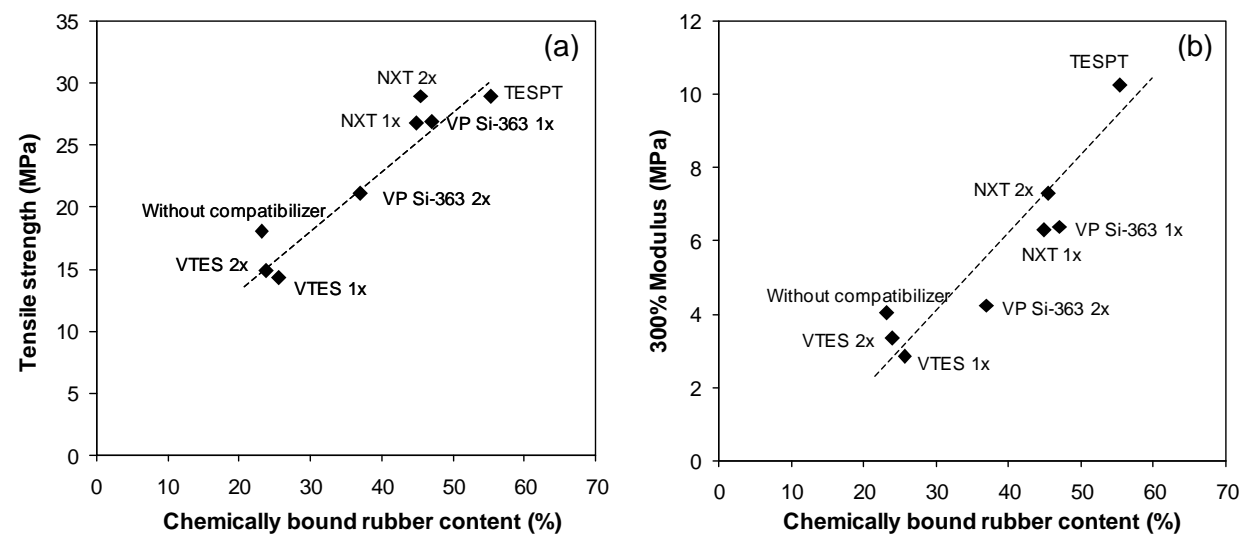

Figure 6.12 Tensile strength (a) and 300\%-modulus (b) of silica-filled NR compounds with different types of silane coupling agents as a function of chemically bound rubber content. 

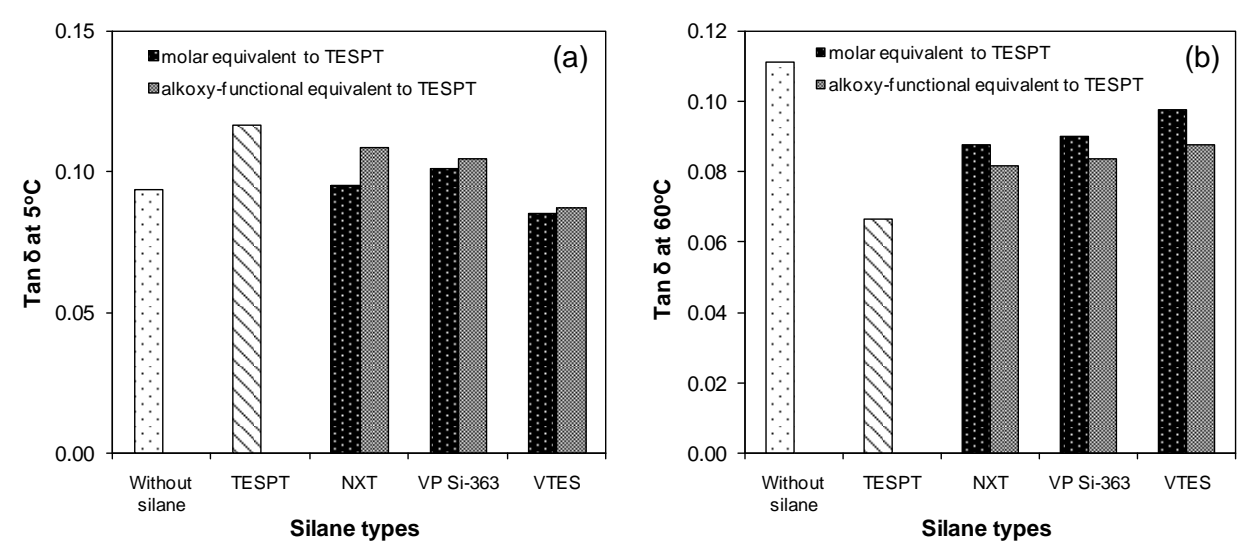

Figure 6.13 Tan $\delta$ values at $5^{\circ} \mathrm{C}(\mathrm{a})$ and $60^{\circ} \mathrm{C}(\mathrm{b})$ of silica-filled NR with different types and amounts of silane coupling agents.

For tire applications or mechanical goods for use under dynamic conditions, dynamic mechanical properties are of great importance. The key tire performances, i.e. wet grip, rolling resistance and wear, are all related to the dynamic mechanical properties of the rubber vulcanizates. Basically, wet grip refers to breaking ability for normal rubber tires ${ }^{[22]}$, and rolling resistance is a measure of the amount of energy consumed over distance travelled. Herein, tan $\delta$ values at $5^{\circ} \mathrm{C}$ and $60^{\circ} \mathrm{C}$ may respectively be used to indicate wet grip and rolling resistance, as shown in Figure 6.13. ${ }^{* *)}$ Figure 6.13(a) shows the tan $\delta$ at $5^{\circ} \mathrm{C}$ of the silica-filled NR compounds with different types of silane coupling agents in which the use of TESPT gives the highest tan $\delta$ at $5^{\circ} \mathrm{C}$ whereas the addition of VTES results in the lowest $\tan \delta$ at $5^{\circ} \mathrm{C}$, close to the value of the compound without silane. The vulcanizates with NXT and VP Si-363 show a higher tan $\delta$ at $5^{\circ} \mathrm{C}$ compared to the one without silane, and the values are increased with increasing concentration of the silanes. The results give a first indication that silica-filled NR vulcanizates with TESPT, NXT and VP Si-363 should provide a better wet grip than that without silane.

The tan $\delta$ values at $60^{\circ} \mathrm{C}$ of the silica-filled NR vulcanizates are shown in Figure 6.13(b). For energy saving tires, this value should be low for reduction of heat build-up or decrease of energy loss of tires, affected by friction and heat developed between the tire

\footnotetext{
${ }^{* *)}$ In another project "Safe Tires-Save Energy" within the department of Elastomer Technology and Engineering at the University of Twente, the correlation between tan $\delta$ at $0^{\circ} \mathrm{C}$ and factual friction performance of tire compounds has been studied: E.M. Cichomski, "Silica-silane reinforced passenger car tire treads: Effect of silica morphology, silica-polymer interface structure and rubber matrix network on tire-performance indicators": PhD-thesis, University of Twente, 29 May 2015. In spite of the commonly accepted assumption that the tan $\delta$ at $0-20^{\circ} \mathrm{C}$ is indicative of wet grip, it has been shown that this has to be taken with great reserve and does not generally hold.
} 
tread and road surface. The results in Figure 6.13(b) clearly show that the silica-filled NR without silane coupling agent shows the highest $\tan \delta$ value at $60^{\circ} \mathrm{C}$ due to poor fillerrubber interactions and thus inferior energy transfer as well as poor reinforcing efficiency. The use of TESPT gives the best low tan $\delta$ at $60^{\circ} \mathrm{C}$ implying the best low rolling resistance of a tire compound. Several factors contribute to this. The best filler-rubber interactions as indicated by the chemically bound rubber content: Figure 6.6(a); and the highest crosslink density as implied by the highest cure torque difference; Figures 6.7-6.9, likely due to the earlier mentioned sulfur "donation" of TESPT. The use of NXT and VP Si-363 lowers the $\tan \delta$ value at $60^{\circ} \mathrm{C}$ compared to the filled rubber with no silane, where both types of silanes give similar tan $\delta$ values. Increasing the NXT and VP Si-363 contents in order to provide equal amounts of alkoxy groups to the reference TESPT slightly decrease the tan $\delta$ at $60^{\circ} \mathrm{C}$, which may be caused by a better filler dispersion.

Based on the overall results, TESPT is the best silane coupling agent in this study for silica-filled NR compounds providing outstanding filler-rubber interaction, mechanical and dynamic properties. The mercaptosilanes NXT and VP Si-363 efficiently reduce the filler-filler interaction, enhance the compound properties, i.e. lower Mooney viscosity and improve cure behavior, but give somewhat inferior mechanical and dynamic properties compared to TESPT, most likely due to a lower degree of crosslinking than for TESPT, which "donates" extra sulfur to the curing package. The difference could probably partly or totally be overcome by adding some extra sulfur to the pertinent formulation. VTES is not an effective silane coupling agent for this sulfur-crosslinked system as it results in no improvement in properties compared to the compound without silane

\subsection{Conclusions}

The comparative study of the compound and vulcanizate properties of the silicafilled NR compounds silanized by four types of silane coupling agents, i.e. TESPT, NXT, VP Si-363 and VTES, shows their different effects on the reinforcement of silica in NR. Among the four silane types, VTES gives the poorest overall properties due to its poor shielding efficiency and the vinyl-group that is difficult to couple with NR rubber chains in the presence of sulfur. The use of NXT and VP Si-363 shows a good improvement in compound properties, i.e. mixing energy, Mooney viscosity, cure behaviors, bound rubber content, and an increase of mechanical and dynamic mechanical properties compared to that without silane, but the properties are inferior to the use of TESPT, mainly due to lower crosslink densities compared with TESPT which has an intrinsic sulfur "donating" effect. This was not further pursued in the context of this chapter. The incorporation of NXT 
silane provides better scorch safety, and use at the same amount of ethoxy-functional groups with reference to TESPT gives vulcanizates with the same level of tensile strength, but poorer modulus and tan $\delta$ at both $5^{\circ} \mathrm{C}$ and $60^{\circ} \mathrm{C}$.

\subsection{References}

1. L.A.E.M. Reuvekamp, J.W. ten Brinke, P.J. Van Swaaij, J.W.M. Noordermeer, Rubber Chem. Technol., 75, 187 (2002).

2. J.W. ten Brinke, S.C. Debnath, L.A.E.M. Reuvekamp, J.W.M. Noordermeer, Comp. Sci. Technol., 63, 1165 (2003).

3. J.W. ten Brinke, P.J. Van Swaaij, L.A.E.M. Reuvekamp, J.W.M. Noordermeer, Kautsch. Gummi Kunstst., 55, 244 (2002).

4. L. Ladouce-Stelandre, Y. Bomal, L. Flandin, D. Labarre, Rubber Chem. Technol., 76, 145 (2003).

5. H. Yan, K. Sun, Y. Zhang, Y. Zhang, J. Appl. Polym. Sci., 94, 2295 (2004).

6. H. Yan, K. Sun, Y. Zhang, Y. Zhang, Y. Fan, J. Appl. Polym. Sci., 94, 1511 (2004).

7. A. Blume, Kautsch. Gummi. Kunstst., 64 (4), 38 (2011).

8. O. Klockmann, The International Tire Exhibition \& Conference 2006, 12-14 September 2006, Akron, United State of America.

9. W. Niedermeier, B. Schwaiger, Kautsch. Gummi. Kunstst., 60, 184 (2007).

10. D.J. Lowe, A.V. Chapman, S. Cook, J.J.C. Busfield, Rubber Chem. Technol., 86, 538 (2003).

11. Y.-T. Shieh, C.-M. Liu, J. Appl. Polym. Sci., 74, 3404 (1999).

12. M. Beltran, C. Mijangos, Polym. Eng. Sci., 40, 1534 (2000).

13. C. Jiao, Z. Wang, Z. Gui, Y. Hu, Eur. Polym. J., 41, 1204 (2005).

14. K. Sirisinha, M. Boonkongkaew, S. Kositchaiyong, Polym. Test., 29, 958 (2010).

15. M.W. Ranny, C.A. Pagano, Rubber Chem Technol., 44, 1080 (1971).

16. P.K. Pal, S.K. De, Polymer, 25, 855 (1984).

17. W. Kaewsakul, K. Sahakaro, W.K. Dierkes, J.W.M. Noordermeer, Rubber Chem. Technol., 85, 277 (2012).

18. H.D. Luginsland, J. Fröhlich, A. Wehmeier, Rubber Chem. Technol., 75, 563 (2002).

19. S. Wolff, M.J. Wang, E.H. Tan, Rubber Chem. Technol., 66, 163 (1993).

20. G.A. Bohm, W. Tomasawski, W. Cole, T. Hogan, Polymer, 51, 2057 (2010).

21. S. Oae. Organic Sulfur Chemistry: Structure and Mechanism, CRC Press, Boca Raton, USA, p.3 (1991).

22. UNECE Regulation No 117, GRRF 71 ${ }^{\text {st }}$ session, September 13-15, 2011. 


\section{Preparation and Characterization of Silane-Grafted Natural Rubber}

Natural rubber (NR) grafted with silane was prepared by melt mixing using 1,1'di(tert- butylperoxy)-3,3,5-trimethylcyclohexane (Luperox® 231XL40) as initiator, and two types of silane: 3-Octanoylthio-1-propyltriethoxysilane (NXT) and 3-mercaptopropyldi(tridecan-1-oxy-13-penta(ethyleneoxide))ethoxysilane (VP Si-363). First, VP Si-363 silane was used to optimize the grafting conditions for preparing the silane-grafted NR. Grafting temperatures were varied in the range of $100-160^{\circ} \mathrm{C}$ at a fixed initiator content of $0.1 \mathrm{phr}$ and of silane $20 \mathrm{phr}$. The VP Si-363 grafted NR at $140^{\circ} \mathrm{C}$ shows infrared absorption peaks at wave numbers of 3270 and $1075 \mathrm{~cm}^{-1}$ assigned respectively to the deformation of $-\mathrm{OH}$ and $\mathrm{Si}-\mathrm{O}-\mathrm{C}$ functional groups. Increase of the grafting temperature to $160^{\circ} \mathrm{C}$ introduces a new absorption peak at $1710 \mathrm{~cm}^{-1}$ of $\mathrm{C}=\mathrm{O}$ bonds in the grafted $\mathrm{NR}$, indicating occurrence of NR degradation at this high temperature. Increase of the initiator concentration in the range of 0.1-1.0 phr gives no positive effect on the grafted amount but leads to a significant increase of gel content: crosslinking. The conditions for grafting the silanes onto $N R$ in an internal mixer were therefore fixed at $140^{\circ} \mathrm{C}$ and initiator concentration of 0.1 phr. NXT- and VP Si-363-grafted NRs were prepared with different silane concentrations at 10 and 20 phr. The purified silane-grafted NRs show infrared absorption peaks at 1075 and $1035 \mathrm{~cm}^{-1}$ which can be assigned respectively to the deformation of Si-O-C and Si-O-Si bonds, and the peak at $3270 \mathrm{~cm}^{-1}$ of O-H deformation. The ${ }^{1} \mathrm{H}$-NMR spectra of both NXT- and VP Si-363-grafted NRs confirm the presence of methylene protons of the alkoxy groups $\left(-\mathrm{O}_{-} \mathrm{CH}_{2}-\mathrm{C}-\right)$ from the fragments of the silanes that were attached to the NR molecules. The use of NXT silane at 10 and 20 phr for the grafting reaction results in 0.66 and 1.32 mol\% of grafted NXT, whereas the use of VP Si363 at 10 and 20 phr gives 0.85 and 1.64 mol\% of grafted VP Si-363. 


\subsection{Introduction}

Silica has a hydrophilic surface by its specific character of a large number of surface silanol groups, i.e. $4.9 \mathrm{OH} . \mathrm{nm}^{-2[1]}$, leading to strong hydrogen bonding between silica particles and poor filler-rubber interactions in non-polar rubbers. In general, a silane coupling agent is directly added together with silica into a rubber during the mixing process to enhance the silica-rubber compatibility. Under suitable mixing conditions, the silane coupling agent undergoes a silanization reaction with the silanol groups on the silica involving primary and secondary reactions. ${ }^{[2]}$ To complete the reaction between silica and bis-(triethoxysilylpropyl)tetrasulfide (TESPT) silane during mixing, the dump temperature of the silica-filled SBR compound should be above $130^{\circ} \mathrm{C}$, but at a temperature above $160^{\circ} \mathrm{C}$ the silane starts to react with rubber to form premature crosslinks. $^{[3]}$ For silica-filled NR compounds with TESPT as coupling agent, a dump temperature is recommended in a range of $135-150^{\circ} \mathrm{C}$ and a higher temperature leads to degradation of the NR. ${ }^{[4]}$ Some sulfur-free silanes with alkoxy functional groups may react with the silanol groups on the silica and so shield the silica surface to make it more hydrophobic. ${ }^{[5]}$ Various silanes show a different effect on the properties of silica-filled rubber. A sulfur-free silane shows a good improvement in the Payne effect, but gives poorer mechanical and dynamic mechanical properties of the silica-filled rubber compared to sulfur-containing silanes such as TESPT. ${ }^{[6]}$

Surface modification of silica is an alternative method to improve silica reinforcement in the rubber matrix. Modification of silica with a silane coupling agent can change the characteristic surface from hydrophilic to hydrophobic, which is achieved by partial blocking of hydroxyl groups on the silica surface. Surface modification with vinyland mercapto-silanes reduces the silica aggregate size without re-agglomeration of the silica, but modification with an amino-silane tends to increase the silica agglomerate size because of its hydrophilicity that leads to formation of hydrogen bonds between particles/aggregates. ${ }^{[7,8]}$ Organic monomers such as styrene, isoprene and butadiene have been used to modify the silica surface to obtain a hydrophobic character through in situ polymerization of the monomers adsorbed on the surface via bi-layers of surfactants. $^{[9-11]}$ The use of organic monomer surface-modified silica in a rubber matrix leads to the improvement of cure behaviors, and tensile and tear properties. ${ }^{[10,11]}$ The plasma polymerization technique has been employed to modify silica by deposition of a thin polymer film on its surface to improve the adhesion between the silica and rubber phases, in which the polymer film can be tailored by choosing the monomer types and reaction conditions. Polyacetylene (PA)-, polypyrrole (PPy)- and polythiophene (PTh)- 
coated silica surfaces showed a less hydrophilic character and less filler-filler interaction compared to untreated silica. ${ }^{[12]}$ The plasma polymerization of acetylene onto the silica surface could improve the silica dispersion in styrene-butadiene rubber (SBR) and enhance the tensile strength and modulus. ${ }^{[13,14]}$ A PA-coated silica-filled SBR/ethylenepropylene-diene rubber (EPDM) blend showed more homogeneous distribution of the filler compared to the untreated one. ${ }^{[15]}$

Silane-modified polymer is another route employed for improvement of silica reinforcement in polymeric materials. Polypropylene (PP) grafted with vinyltriethoxysilane (VTES) was used in PP/silica nanocomposites in which the grafted PP could attach to the silica surface through VTES molecules, leading to a decrease of chain mobility and diffusion as proven by a shift of crystallization and melting behaviors to a higher temperature. ${ }^{[16]}$ A liquid low molecular weight polybutadiene (PB) was grafted with mercaptopropyltrimethoxysilane (MPS) through radical addition of the thiol group to the double bond on polymer molecule at $75^{\circ} \mathrm{C}$ and the MPS-grafted PB was silanized onto silica surfaces at $135^{\circ} \mathrm{C}$, allowing the alkoxy-groups of the silane fragment to react with the silanol groups of the silica. ${ }^{[17]}$ Modified-silica with the grafted PB showed a decrease of the number of $\mathrm{OH}$-groups on the surface to the same level as that of silica with TESPT, due to the influence of the shielding effect of the grafted PB. The decrease of hydrophilic character of the surface modified silica further lead to a reduction of filler-filler interaction and an improvement of filler dispersion in the rubber matrix. ${ }^{[18]}$ The use of MPS silanemodified silica enhanced the final properties like tensile strength, but to a lesser extent than the conventional use of TESPT as coupling agent during rubber mixing and silanization.

In Chapter 6, the use of 3-octanoylthio-1-propyltriethoxysilane (NXT) and 3mercaptopropyl-di(tridecan-1-oxy-13-penta(ethyleneoxide))ethoxysilane (VP Si-363) in silica-filled NR improved both compound and vulcanizate properties, although the use of TESPT still provided the best overall properties. However, the grafting of NXT and VP Si363 through their thiol groups onto the NR molecule is still of interest, and this Chapter 7 reports a study on such silane-grafted NRs. The effects of grafting temperature and initiator concentration are investigated in order to find a suitable grafting condition. Then, the optimized condition is applied to prepare NR grafted with silanes using 10 and $20 \mathrm{phr}$ of NXT and VP Si-363 silanes. The silane-grafted NRs are characterized by FourierTransform Infrared (FTIR) and Proton Nuclear Magnetic Resonance ( ${ }^{1} \mathrm{H}$ NMR) spectroscopic techniques. 


\subsection{Experimental}

\subsubsection{Materials}

Natural rubber was Ribbed Smoked Sheet (RSS) \#3, locally produced in Thailand. Two types of silane coupling agents; 3-Octanoylthio-1-propyltriethoxysilane (NXT) (Momentive, USA) and 3-Mercaptopropyl-di(tridecan-1-oxy-13penta(ethyleneoxide))ethoxysilane (VP Si-363) (Evonik, Germany), and 1,1'-di(tertbutylperoxy)-3,3,5-trimethylcyclohexane (Luperox® 231XL40) (Arkema, USA) 40\% extended on calcium carbonate and silica, were used for the melt grafting reactions. The structure of the initiator is shown in Figure 7.1 and the silane coupling agent details are as previously given in Table 6.1 in Chapter 6.<smiles>CC1CC(C)(C)CC(OOC(C)(C)C)(OOC(C)(C)C)C1</smiles>

Figure 7.1 Structure of 1,1'-di(tert-butylperoxy)-3,3,5-trimethylcyclohexane (Luperox® 231XL40).

\subsubsection{Preparation of silane-grafted NR}

NR was mixed with the silane coupling agent and the initiator in an internal mixer, Brabender ${ }^{\circledR}$ 50EHT (Brabender ${ }^{\circledR} \mathrm{GmbH}$ \& Co.KG, Germany), for 12 minutes with a rotor speed of $60 \mathrm{rpm}$ using the formulations and mixing steps as shown in Tables 7.1 and 7.2, respectively. For the grafting conditions study, VP Si-363 was used to investigate the effect of grafting temperatures in the range of $100-160^{\circ} \mathrm{C}$ and of the initiator concentrations in the range of 0.1-1.0 phr. The most suitable grafting temperature and initiator concentration were then selected for preparing the VP Si-363- and NXT-grafted NRs by using silane contents at 10 and 20 phr. 
Table 7.1 Formulations for preparing the silane-grafted NRs.

\begin{tabular}{lcccc}
\hline \multirow{2}{*}{ Chemicals } & \multicolumn{4}{c}{ Amount (phr) } \\
\cline { 2 - 5 } & $\begin{array}{c}\text { Varying temp. } \\
100-160^{\circ} \mathrm{C}^{*}\end{array}$ & $\begin{array}{c}\text { Varying initiator } \\
\text { contents }\end{array}$ & $\begin{array}{c}\text { Varying types \& amounts of } \\
\text { silane }\end{array}$ \\
\hline RSS3 & 100.0 & 100.0 & 100.0 & 100.0 \\
VP Si-363 & 20.0 & 20.0 & $10.0,20.0$ & - \\
NXT & - & - & - & $10.0-20.0$ \\
Luperox® 231XL40 & 0.1 & $0.1-1.0$ & 0.1 & 0.1 \\
\hline * The initial temperature setting of the mixer & & &
\end{tabular}

${ }^{*}$ The initial temperature setting of the mixer.

Table 7.2 Mixing procedures for preparing the silane-grafted NRs.

\begin{tabular}{lc}
\multicolumn{1}{c}{ Grafting procedures } & $\begin{array}{c}\text { Cumulative time } \\
\text { (mins.) }\end{array}$ \\
\hline - NR mastication & 0 \\
- Addition of silane coupling agent (NXT or VP Si-363) & 1 \\
- Addition of Luperox® 231XL40 (initiator) & 2 \\
- Discharge & 12 \\
\hline
\end{tabular}

\subsubsection{Characterization of silane-grafted NRs}

\subsubsection{Gel content}

$0.5 \mathrm{~g}$ of the resulting materials of the melt grafting reactions was cut into small pieces and dissolved in $50 \mathrm{ml}$ of toluene for $72 \mathrm{hr}$ under continuous stirring. The mixture was then filtered through a filter paper to separate the gel component out of the solution, and the gel part was dried in an oven for $24 \mathrm{hr}$ at $107^{\circ} \mathrm{C}$. The gel content was calculated by using Equation (7.1):

$$
\text { Gel content }(\%)=\frac{W_{g}}{W_{s}} \times 100
$$

where $W_{g}$ is the weight of the dried gel component, and $W_{s}$ is a weight of grafted rubber sample.

\subsubsection{Structural characterization by Fourier-Transform Infrared Spectroscopy (FTIR)}

An Attenuated Total Reflection (ATR) - FTIR spectrometer (Tensor 27, Bruker) was used to characterize the purified grafted NR samples. Purification of the silane- 
grafted NR samples was performed by dissolving the materials in toluene under continuous stirring for $72 \mathrm{hr}$, then filtering to remove any insoluble part, and subsequently precipitating the soluble part in ethanol to remove any free or ungrafted silane. The purified sample was finally dried in an oven at $60^{\circ} \mathrm{C}$ for $24 \mathrm{hr}$ and kept in a descicator before the analysis. The extent of silane grafting $(R)$ onto the NR molecules was calculated by using the peak height ratios according to Equation (7.2): ${ }^{[19]}$

$$
R_{1075}=\frac{A_{1075}}{A_{1375}} \quad \text { and } \quad R_{1035}=\frac{A_{1035}}{A_{1375}}
$$

where $R_{1075}$ and $R_{1035}$ are the extents of silane-grafted NR at $1075 \mathrm{~cm}^{-1}$ and $1035 \mathrm{~cm}^{-1}$, repectively, $A_{1075}$ and $A_{1035}$ are the peak heights of Si-O-C formation at $1075 \mathrm{~cm}^{-1}$ and $1035 \mathrm{~cm}^{-1}$, respectively, and $A_{1375}$ is the peak height of $-\mathrm{CH}_{3}$ in NR at $1375 \mathrm{~cm}^{-1}$.

7.2.3.3 Structural characterization by Proton Nuclear Magnetic Resonance ('H-NMR) Spectroscopy

Purified grafted NR samples were prepared using the same method as described in Section 7.2.3.2 for FTIR. The purified and dried grafted NR samples were dissolved in deuterated chloroform $\left(\mathrm{CDCl}_{3}\right)$ and characterized by NMR spectroscopy (Varian Unity Inova $500 \mathrm{MHz}$, Varian). The amount of VP Si-363 grafted onto NR molecules in mol\% can be quantitatively analyzed by using Equation (7.3):

$$
\text { mol\% of grafted VP Si-363 on NR }=\frac{A_{4.0-3.5} / 46}{\left(A_{4.0-3.5} / 46\right)+A_{5.1}} \times 100
$$

where $A_{4.0-3.5}$ is the integrated peak area of methylene protons of the alkoxy groups (-SiO- $\underline{\mathrm{C}}_{2}-\mathrm{CH}_{3}$ and $\left.-\mathrm{Si}-\mathrm{O}-\left(\mathrm{C}_{2} \underline{\mathrm{H}}_{4} \mathrm{O}\right)_{5}-\mathrm{C}_{2}-\mathrm{C}_{12} \mathrm{H}_{25}\right)$ of the VP Si-363 in the range 4.0-3.5 ppm,

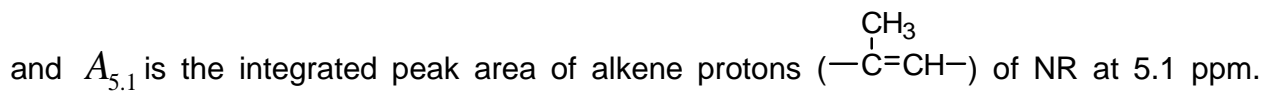
There are a total of 46 alkoxymethylene protons in each VP Si-363 molecule (as the structure shown in Table 6.1), and 1 alkene proton in each isoprene unit.

The amount of NXT grafted onto NR molecules in mol\% can be quantitatively analyzed by using Equation (7.4): 


$$
\text { mol\% of grafted NXT on } N R=\frac{A_{3.8} / 6}{\left(A_{3.8} / 6\right)+A_{5.1}} \times 100
$$

where $A_{3.8}$ is the integrated peak area of methylene protons of the alkoxy group (-Si-O$\underline{\mathrm{C}}_{2}-\mathrm{CH}_{3}$ ) of the NXT at $3.8 \mathrm{ppm}$, and $A_{5.1}$ is the integrated peak area of alkene protons $\mathrm{CH}_{3}$

$(-\mathrm{C}=\mathrm{CH}-)$ of $\mathrm{NR}$ at $5.1 \mathrm{ppm}$. There are 6 alkoxy-methylene protons in each NXT molecule and 1 alkene proton in each isoprene unit.

\subsubsection{Grafting efficiency of silane-grafted NR}

Based on mol\% of grafted silane on the NR obtained from the calculation according to Equations (7.3) and (7.4), the weight\% of grafted silane can be calculated by using Equation (7.5):

W t $\%$ of grafted silane on $N R=\frac{\text { mol\% } \%_{\text {silane }} \times M W_{\text {silane }}}{\left(\text { mol\% }_{\text {silane }} \times M W_{\text {silane }}\right)+\left(\text { mol\% }_{N R} \times M W_{N R}\right)} \times 100$

where mol\%silane is the mol\% of grafted silane on $\mathrm{NR}, \mathrm{MW}_{\text {silane }}$ is the molecular weight of the silane, mol\%NR is the mol\% of $N R$ and $M_{N R}$ is the molecular weight of the $N R$ repeating unit $(68 \mathrm{~g} / \mathrm{mol})$.

Then, the grafting efficiency can be calculated according to Equation (7.6):

$$
\text { Grafting efficiency }(\%)=\frac{w t \% \text { of grafted silane on } N R}{w t \% \text { of silane used for the reaction }} \times 100
$$

\subsubsection{Elemental analysis by Scanning Electron Microscopy - Energy Dispersive X-Ray Spectroscopy (SEM-EDX)}

The purified silane-grafted NRs were analyzed for the chemical elements carbon (C), oxygen (O) and silicon ( $\mathrm{Si}$ ) by using SEM-EDX spectroscopy (Quanta 400, FEI). 


\subsection{Results and discussion}

\subsubsection{Characterization of NR, NXT and VP Si-363 as starting materials}

Before the grafting reaction, the starting materials: rubber and silanes were characterized for their chemical structures by FTIR and ${ }^{1} \mathrm{H}$-NMR spectroscopy in order to have the reference spectra, as respectively shown in Figures 7.2 and 7.3.

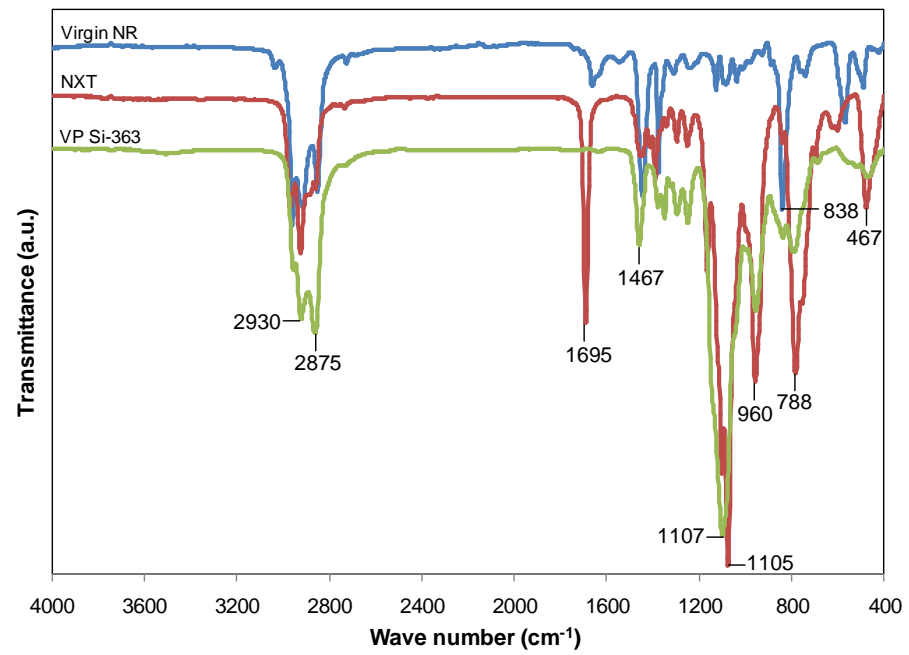

Figure 7.2 ATR-FTIR spectra of NR, NXT and VP Si-363.

Figure 7.2 shows the FTIR spectra of the starting materials: unmodified NR, NXT and VP Si-363 silane coupling agents, in the wave number range of $4000-400 \mathrm{~cm}^{-1}$. The assignments for the main absorption bands of NR and the two types of silane are listed in Table 7.3. ${ }^{[20-23]}$

Table 7.3 Wave number and assignments for NR, NXT and VP Si-363.

\begin{tabular}{cl}
\hline Wave number $\left(\mathbf{c m}^{-1}\right)$ & Functional groups \\
\hline 2930 & C-H asymmetric stretching \\
2875 & C-H symmetric stretching \\
1695 & C=O stretching \\
1670 & C=C stretching \\
1467 & C-H bending \\
$\sim 1100$ & Si-O-C stretching \\
960 & Si-O-C stretching \\
838 & C-H out of plane deformation of NR \\
788 & Si-O-C vibration of alkoxy-silane \\
467 & O-Si-O deformation \\
\hline
\end{tabular}


The characteristic peaks of NR are displayed at the wave numbers of 1670 and $838 \mathrm{~cm}^{-1}$ which are assigned respectively to the $\mathrm{C}=\mathrm{C}$ stretching vibration and $=\mathrm{C}-\mathrm{H}$ out of plane deformation of the isoprene unit in NR molecules. For the NXT mercaptosilane, in addition to the $\mathrm{C}-\mathrm{H}$ stretching vibrations at 2930 and $2875 \mathrm{~cm}^{-1}$ and $\mathrm{C}-\mathrm{H}$ bending deformations at $1467 \mathrm{~cm}^{-1}$, strong characteristic peaks of NXT are observed at the wave number of $1695 \mathrm{~cm}^{-1}$ due to $\mathrm{C}=\mathrm{O}$ deformation of the blocking group, and at $\sim 1100,960$ and $788 \mathrm{~cm}^{-1}$ due to the vibrations of the Si-O-C functional group of the silane. Furthermore, the spectrum of NXT shows an absorption peak at $467 \mathrm{~cm}^{-1}$ assigned to OSi-O deformation. ${ }^{[21]}$ VP Si-363 is a mercapto-silane with one ethoxy group and two long bulky groups comprising of long aliphatic chains and ethylene oxide units. The spectrum of VP Si-363 shows strong absorption peaks of the Si-O-C functional group at the wave numbers of $\sim 1100,960$ and $788 \mathrm{~cm}^{-1}$.

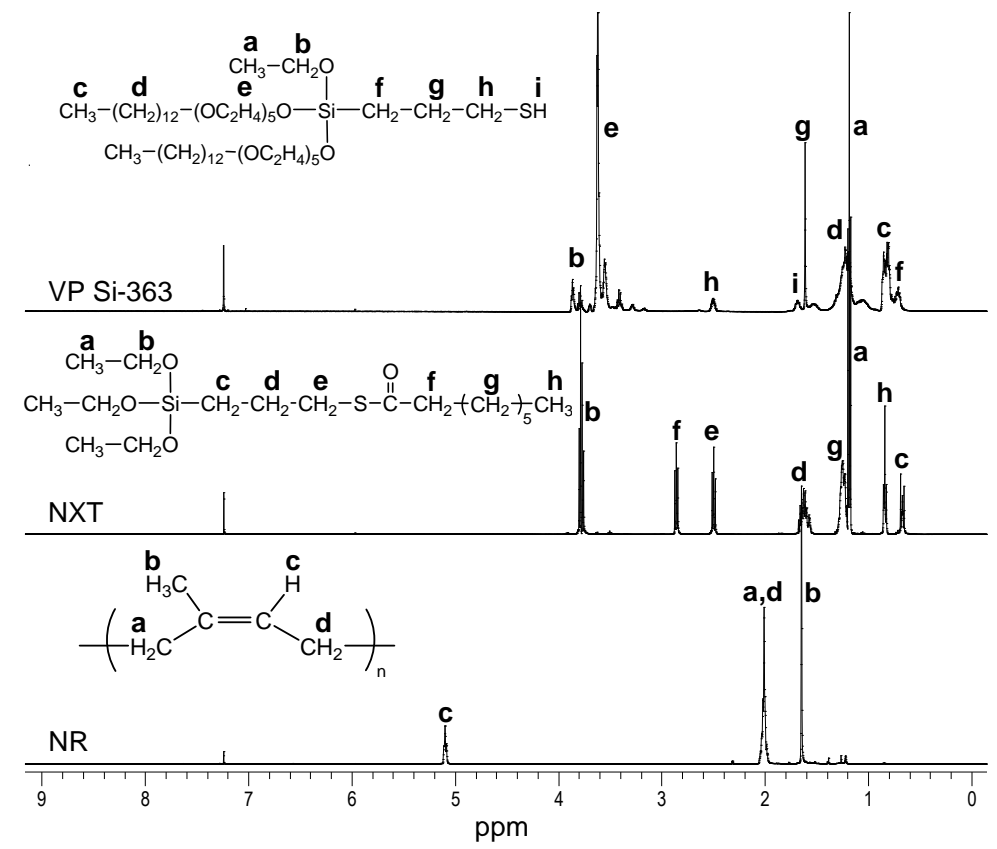

Figure $7.3{ }^{1} \mathrm{H}-\mathrm{NMR}$ spectra of NR, NXT and VP Si-363.

The ${ }^{1} \mathrm{H}-\mathrm{NMR}$ spectra of pure NR, NXT and VP Si-363 silanes are comparatively shown in Figure 7.3. NR has specific absorption bands at chemical shifts of 5.1, 2.0 and $1.6 \mathrm{ppm}$ assigned to the alkene proton $(=\mathrm{CH})$, the methylene proton $\left(-\mathrm{CH}_{2}-\right)$ and methyl protons $\left(-\mathrm{CH}_{3}\right)$ of polyisoprene, respectively. The NXT and VP Si-363 silanes have ${ }^{1} \mathrm{H}-$ NMR spectra showing more characteristics peaks assigned to different proton types in 
their structures as indicated in Figure 7.3. The main peaks of methylene- and methylprotons of the ethoxy groups appear at 3.8 and $1.2 \mathrm{ppm}$, respectively. ${ }^{[24,25]}$ The ${ }^{1} \mathrm{H}-\mathrm{NMR}$ spectrum of NXT shows the absorption bands of the methylene proton adjacent to the sulfur atom at $2.45 \mathrm{ppm}$ and to the carboxyl-group at $2.8 \mathrm{ppm}$. The methylene-proton adjacent to $-\mathrm{Si}$ is displayed at $0.7 \mathrm{ppm}$. The long alkoxy-groups of VP Si-363 silane displays a strong specific absorption band at a chemical shift of $3.6 \mathrm{ppm}$ of the methyleneproton of the ethylene oxide unit $\left(-\mathrm{OCH}_{2} \mathrm{CH}_{2}-\right)$. Assignments for each peak are detailed in Figure 7.3. Due to the presence of several methylene protons (- $\left.\mathrm{CH}_{2}^{-}\right)$attached to oxygen atoms in the structure of VP Si-363, its absorption bands in the region of 4.0-3.5 ppm are more complicated compared to the spectrum of NXT. A small change in the chemical environment around $-\mathrm{O}-\mathrm{CH}_{2}$ - causes a slight shift of the peak and so more peaks associated with the methylene protons of the ethoxy and ethylene oxide groups are observed for the case of VP Si-363.

\subsubsection{Preparation and characterization of silane-grafted NR}

Grafting of silane onto polymers has been reported employing different reaction conditions. Silane grafted onto polybutadiene was done in solution under nitrogen atmosphere. ${ }^{[17,18,26]}$. The solution state is known to provide a better control of the reaction compared to the melt state, but it is environmentally unfriendly and provides only a small amount of the grafted-polymer per batch. The reaction in solution is therefore not preferred for industrial scale. VTES grafted onto styrene butadiene rubber (SBR) had been studied in the latex state using benzoic peroxide as initiator. ${ }^{[27]}$ Furthermore, silane grafting in the melt state has been reported for vinyl-silane-grafted polyolefins. ${ }^{[28,29]}$ For practical purposes, the present work therefore prepared silane-grafted NR by melt mixing in an internal mixer using 1,1'-di(tert-butylperoxy)-3,3,5-trimethylcyclohexane or Luperox ${ }^{\circledR}$ $231 X L 40$ as initiator. The half-life time of Luperox® 231 at $140^{\circ} \mathrm{C}$ is about 5 minutes which is shorter than that of dicumyl peroxide (DCP) with its half-life time of about 30 minutes at the same temperature. The shorter half-life time of Luperox $\circledast 231$ allows the grafting process to be conducted at lower temperature and shorter reaction time compared to the use of DCP to prevent thermal degradation of the NR.

\subsubsection{Effect of grafting temperature and initiator concentration}

Grafting conditions, i.e. the grafting temperature and initiator concentration, were investigated by using VP Si-363 silane at 20 phr. First, the grafting temperature was varied in the range of 100 to $160^{\circ} \mathrm{C}$ while the initiator content was fixed at $0.1 \mathrm{phr}$. Then, 
the selected grafting temperature was fixed and the initiator contents were varied from 0.1 to $1.0 \mathrm{phr}$. The resulting materials were characterized for gel content and structure to confirm the presence of grafted silane on the NR.

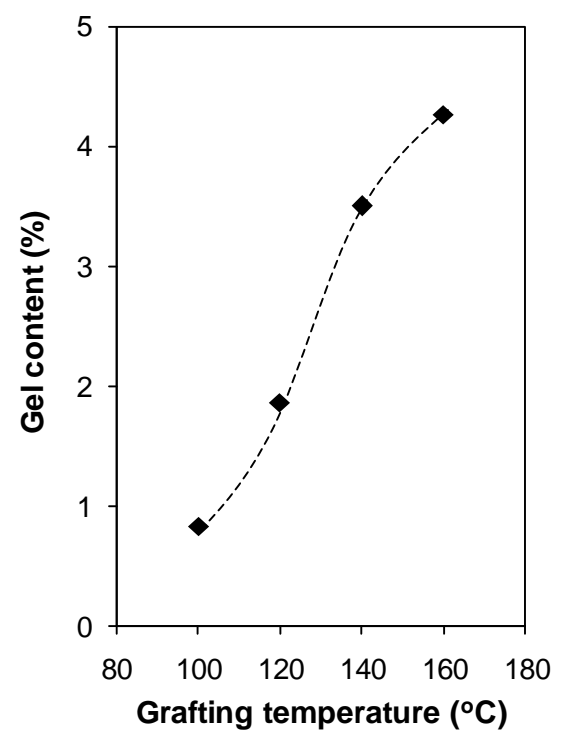

Figure 7.4 Gel content of VP Si-363-grafted NR vs. different grafting temperatures.

Gel contents of VP Si-363-grafted NRs as shown in Figure 7.4 increase with increasing grafting temperature, caused by the increases of the reaction efficiency of the initiator as well as the possibility of the macroradicals to form crosslinks. As mentioned previously, Luperox® 231 has the half-life time of $5 \mathrm{~min}$ at $140^{\circ} \mathrm{C}$. Increasing the reaction temperature therefore generates more free radicals in shorter time, and causes a crosslinking reaction between the rubber molecules in competition with the grafting reaction, leading to the increase of gel content in the material. 


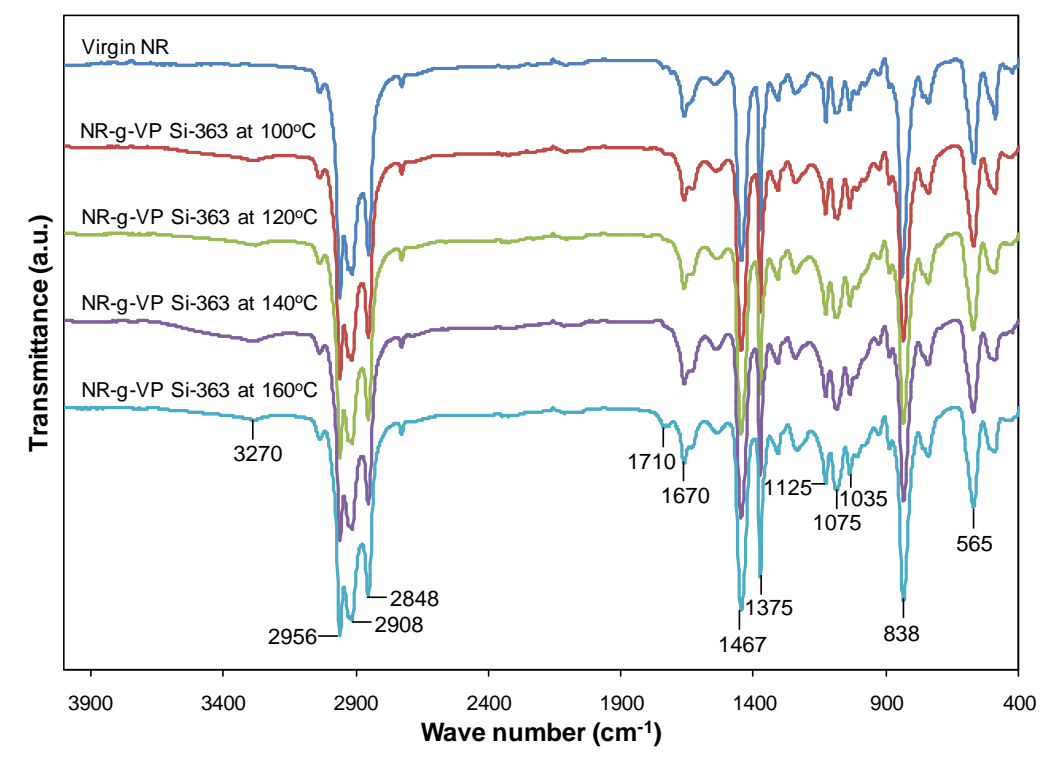

Figure 7.5 ATR-FTIR spectra of VP Si-363-grafted NR with different grafting temperatures.
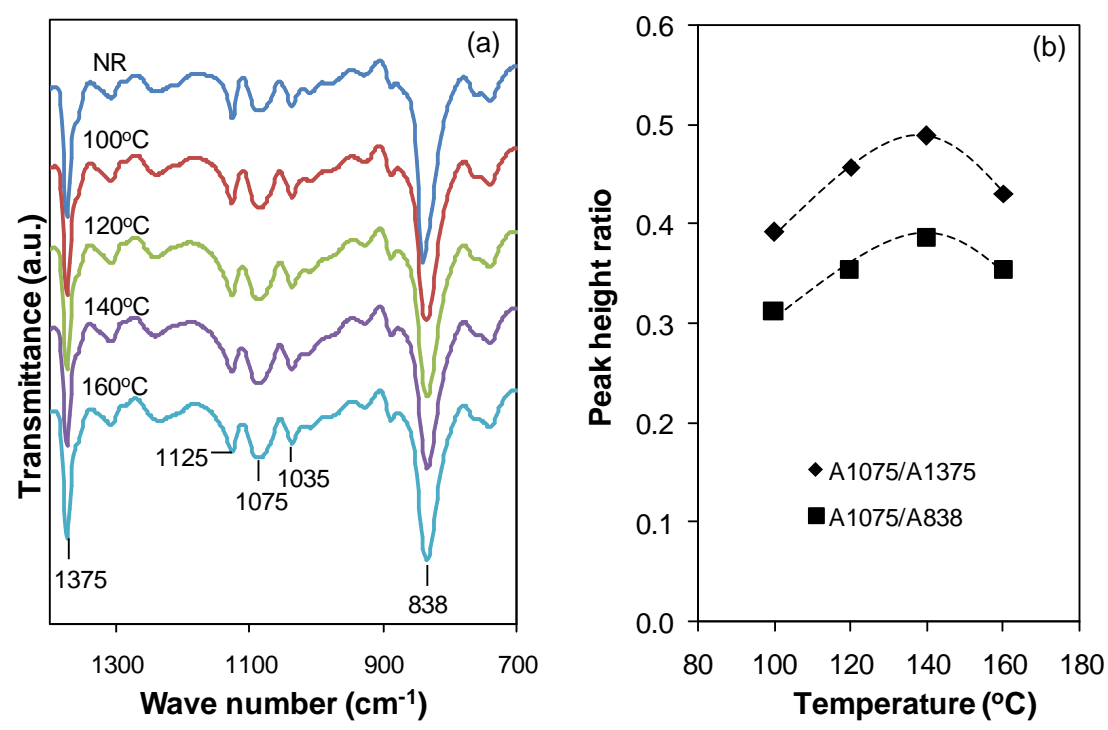

Figure 7.6 FTIR spectra in the range of $1400-700 \mathrm{~cm}^{-1}$ (a) and peak height ratios (b) of VP Si-363-grafted NR with different grafting temperatures.

From the FTIR spectra in Figure 7.5, the peak intensity at the wave number of $1075 \mathrm{~cm}^{-1}$ associated with the vibration of Si-O-C bonds is increased when the grafting 
temperature was increased. Jiao et al. ${ }^{[23]}$ studied silane grafted ethylene-octene copolymer and reported that the peaks at 1167, 1105, 1082 and $958 \mathrm{~cm}^{-1}$ could be assigned to the deformation of $\mathrm{Si}-\mathrm{O}-\mathrm{CH}_{2} \mathrm{CH}_{3}$ of the silane coupling agent. In this finger print region as seen in Figures 7.5 and 7.6(a), virgin NR also shows absorption bands in the range of $1400-700 \mathrm{~cm}^{-1}$, so it is not easy to identify the Si-O-C deformations of the silane grafted NR. However, the peak intensity at $1075 \mathrm{~cm}^{-1}$ relative to the neighboring peaks at 1125 and $1035 \mathrm{~cm}^{-1}$ has changed indicating that some reactions have taken place between silane and rubber. It can be noticed that the intensity of the peak at 1075 $\mathrm{cm}^{-1}$ is becoming stronger when the reaction temperature is increased especially at $140^{\circ} \mathrm{C}$. This peak is due to the Si-O-C deformation which confirms the presence of silane attached to the NR molecules. A weak peak at $3270 \mathrm{~cm}^{-1}$ is also observed in the silanegrafted NR that can be assigned to Si-O-H deformations due to hydrolysis of the alkoxygroup in the silane structure during the grafting reaction or during the post-treatment. The VP Si-363-grafted NR prepared at $160^{\circ} \mathrm{C}$ additionally shows an absorption peak at 1710 $\mathrm{cm}^{-1}$ due to the vibration of a carbonyl group $(\mathrm{C}=\mathrm{O})$, suggesting the occurrence of thermal degradation of the NR. The peak height ratios at $A_{1075} / A_{1375}$ and $A_{1075} / A_{838}$ as shown in Figure 7.6(b) are used to indicate the extent of the grafting reaction. The increase of grafting temperature from $100^{\circ} \mathrm{C}$ to $140^{\circ} \mathrm{C}$ increases the peak height ratios, but the further increase of temperature to $160^{\circ} \mathrm{C}$ decreases that ratios due to the competitive crosslinking and degradation reactions. Based on the peak height ratios and the absence of degradation, the grafting temperature at $140^{\circ} \mathrm{C}$ seems the most suitable condition, and this temperature was therefore selected for a further study on the effect of initiator concentration. 


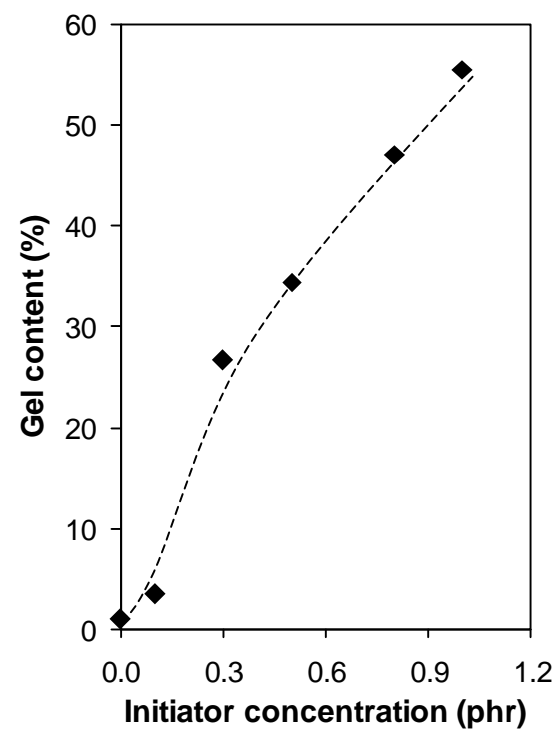

Figure 7.7 Gel content of VP Si-363-grafted NR with different initiator concentrations.

Increasing of initiator concentrations for the melt mixing of silane and NR leads to an increase of gel content in the grafted NR as shown in Figure 7.7. The gel content sharpy increases when $0.3 \mathrm{phr}$ of initiator was used and progressively rises with a further increase of peroxide content. When a high amount of initiator is used, a large number of reactive free radicals is generated and leads to a faster rate of termination as well as a crosslinking reaction without enhancing the grafting efficiency. The effect of increasing initiator concentration on the grafting reaction has also been reported to not only lead to an increase of the termination rate, but also to a slight decrease of grafting efficiency. ${ }^{[30,31]}$ With a high amount of reactive free radicals in the system, rubber crosslinks can easily be formed through C-C linkages generating gel or an insoluble part of the rubber network, and causing more difficulties for the grafting reaction to take place. 


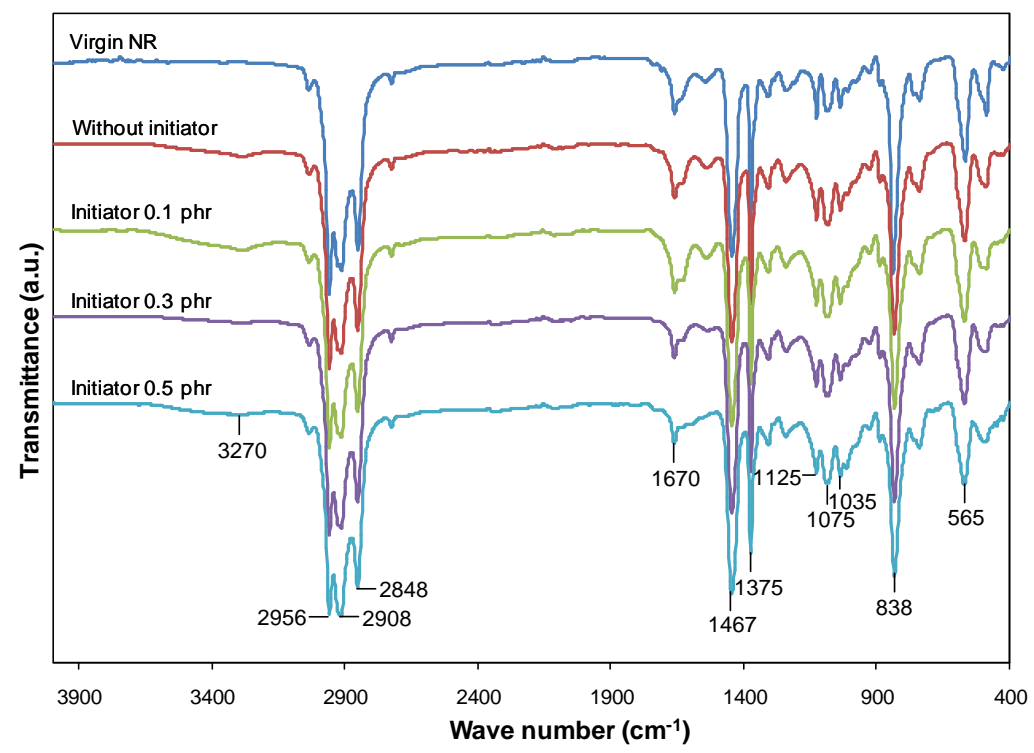

Figure 7.8 ATR-FTIR spectra of VP Si-363-grafted NR with different initiator concentrations at $140^{\circ} \mathrm{C}$.
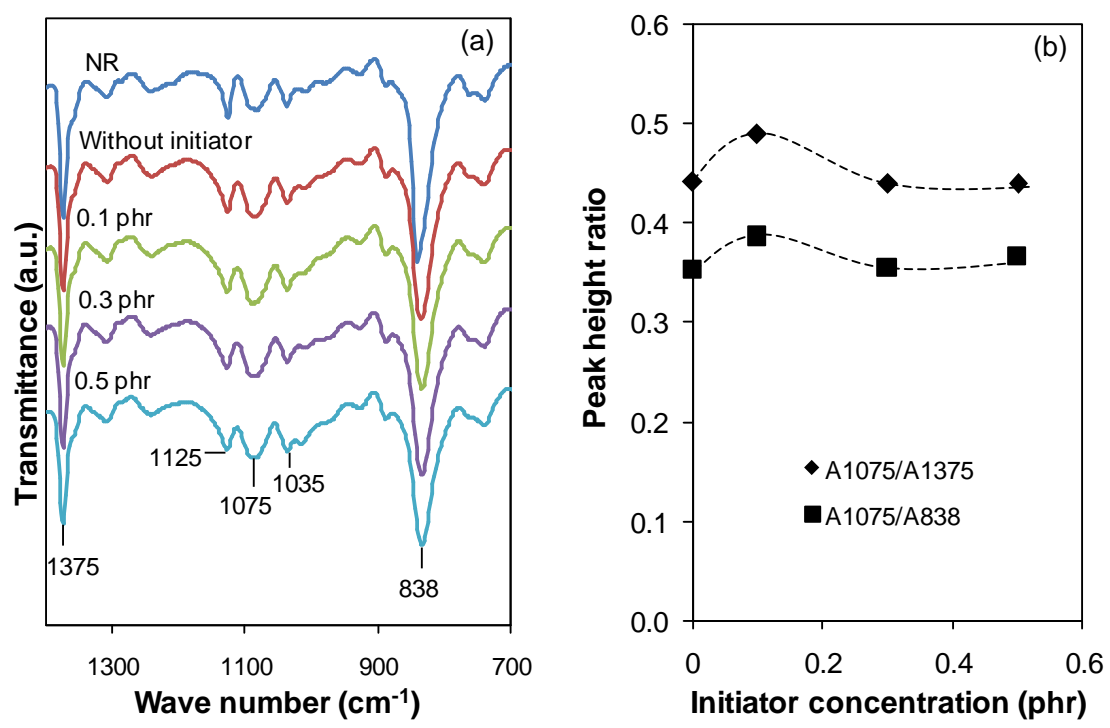

Figure 7.9 FTIR spectra in the range of $1400-700 \mathrm{~cm}^{-1}$ (a) and peak height ratios (b) of VP Si-363-grafted NR with different initiator concentrations. 
The purified silane-grafted NRs that were prepared either without or with initiator show a change of the peak intensity at $1075 \mathrm{~cm}^{-1}$ of $\mathrm{Si}-\mathrm{O}-\mathrm{C}$ bonds relative to the neighboring peaks at 1127 and $1035 \mathrm{~cm}^{-1}$, compared to that of the virgin NR. A slight increase of the peak height ratios of $A_{1075} / A_{1375}$ and $A_{1075} / A_{838}$ when 0.1 phr of initiator was added into the grafting system compared to that without initiator is shown in Figures 7.8 and 7.9, indicating a slightly larger extent of grafting reactions. The grafted materials also show a weak peak of $-\mathrm{OH}$ at a wave number of $3270 \mathrm{~cm}^{-1}$ suggesting the presence of hydrolyzed alkoxy groups. However, the further increase of initiator concentration decreases the peak height ratio to more or less the same level as that of the material without initiator as seen in Figure 7.9(b), due to the fact that the high amount of generated radicals tends to generate more crosslinking between the rubber chains than grafting of silane onto the rubber molecules, as seen in the high gel contents in Figure 7.7. Moreover, the absorption intensity at $1670 \mathrm{~cm}^{-1}$ due to the $\mathrm{C}=\mathrm{C}$ deformation of the NR molecules tends to decrease with increasing initiator concentrations. The decreased $C=C$ double bonds are most likely due to the crosslinking reaction as well as the grafting reaction in NR via the addition at the unsaturated $C=C$ bonds in the NR structure. In this case, the decrease of $\mathrm{C}=\mathrm{C}$ peak intensity at higher initiator content shall be mainly because of the crosslinking reaction, in correspondence with the increasing gel content in Figure 7.7.

Based on the varied temperatures and initiator contents, a grafting temperature at $140^{\circ} \mathrm{C}$ with $0.1 \mathrm{phr}$ of initiator was selected to prepare the silane-grafted NR's by using 10 and 20 phr of VP Si-363 and NXT. The resulting products will be additionally characterized by ${ }^{1} \mathrm{H}-\mathrm{NMR}$ and elemental analysis.

\subsubsection{VP Si-363-grafted NR}

The grafting of NR with VP Si-363 using two different silane loadings; 10 and 20 phr, results in the materials having the FTIR and ${ }^{1} \mathrm{H}-\mathrm{NMR}$ spectra as shown in Figures 7.10 and 7.11, respectively. The spectrum of virgin NR is also included in the figures for comparison. 


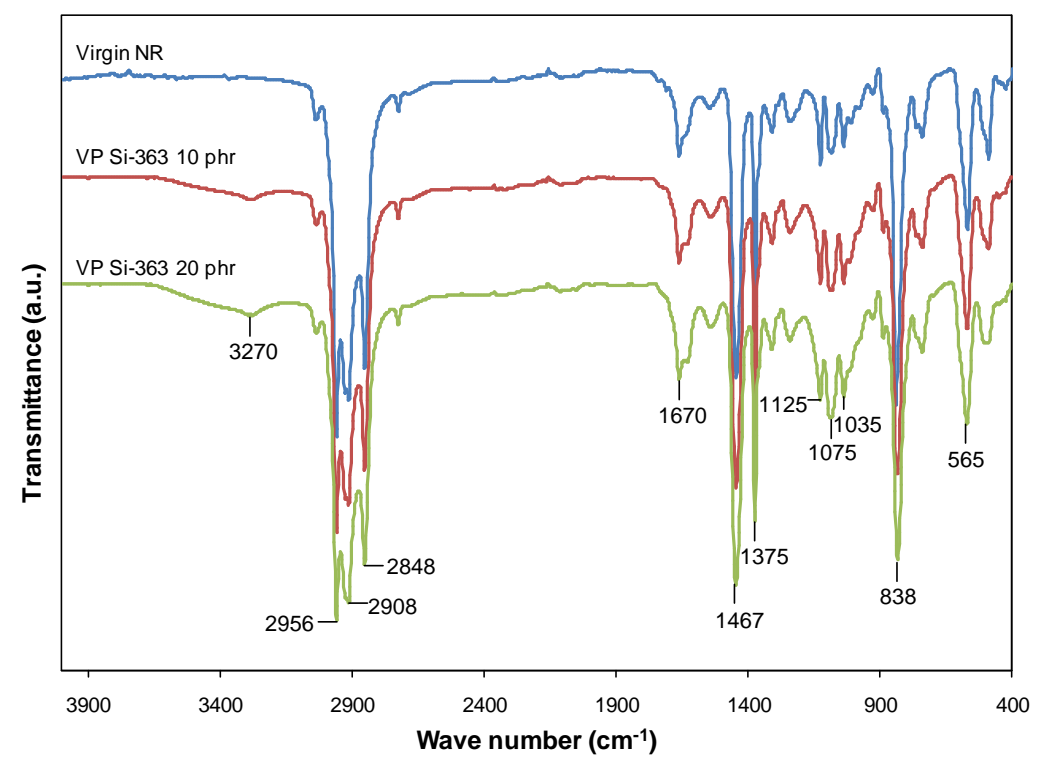

Figure 7.10 ATR-FTIR spectra of virgin NR and NR-grafted with VP Si-363 at 10 and 20 phr.

Similar to Figure 7.5, the NR grafted with VP Si-363 shows the characteristic peaks of silane on NR at the wave number of $1075 \mathrm{~cm}^{-1}$ which is assigned to the Si-O-C bond, and at $1035 \mathrm{~cm}^{-1}$ due to the Si-O-Si vibration. The Si-O-Si bond is the result of a condensation reaction between the alkoxy groups. For comparison, the extent of silane grafting $(\mathrm{R})$ can be calculated by using the peak height ratios taking the peak of the $-\mathrm{CH}_{3}$ vibration in the NR structure at $1375 \mathrm{~cm}^{-1}$ as an internal standard, as also applied for other silane grafted polymers. ${ }^{[19]}$ The calculated results are given in Table 7.4. With increasing silane content used for the reaction, the peak height ratios at the wave numbers of 1075 $\mathrm{cm}^{-1}$ and $1035 \mathrm{~cm}^{-1}$ relative to $1375 \mathrm{~cm}^{-1}$ increase. On comparing with virgin $\mathrm{NR}$, the extent of silane grafting or R-values of the grafted NR clearly increase with increasing silane content used for the melt grafting, providing the proof that the silane is successfully grafted onto the NR molecules with, to some extent, the alkoxy-groups reacted with each other to form siloxane linkages (Si-O-Si) during the grafting reaction. Moreover, the grafted NRs also show a weak intensity peak of $-\mathrm{OH}$ at $3270 \mathrm{~cm}^{-1}$, that may indicate the presence of Si-OH due to hydrolysis of the alkoxy-group of the silane by moisture. 


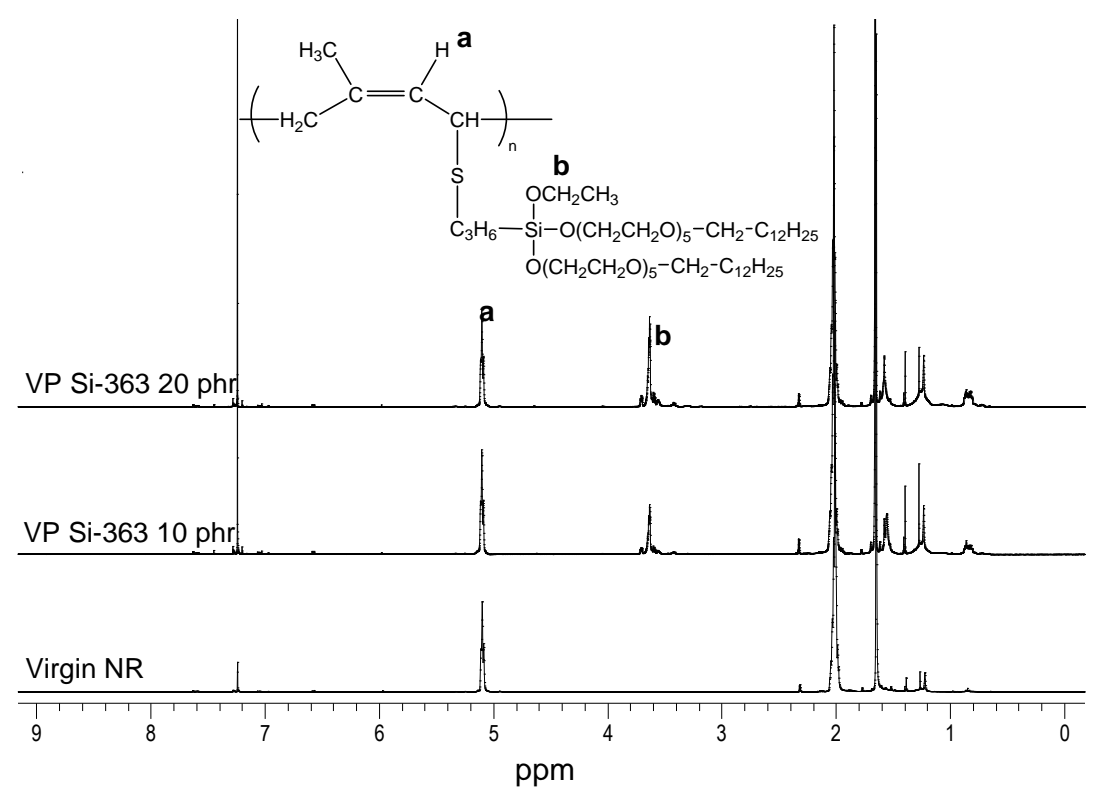

Figure $7.11{ }^{1} \mathrm{H}-\mathrm{NMR}$ spectra of virgin NR and NR-grafted with VP Si-363 at 10 and 20 phr.

Table 7.4 The extent of VP Si-363 grafting (R) onto NR molecules from ATR-FTIR and mol\% of VP Si-363 on NR molecules from ${ }^{1} \mathrm{H}-\mathrm{NMR}$.

\begin{tabular}{|c|c|c|c|}
\hline \multirow{2}{*}{$\begin{array}{c}\text { Amount of VP Si- } \\
363 \text { (phr) }\end{array}$} & \multicolumn{2}{|c|}{ R value from ATR-FTIR } & \multirow{2}{*}{$\begin{array}{c}\text { Mol\% of VP Si- } \\
363 \\
\text { from }{ }^{1} \mathrm{H} \text { NMR }\end{array}$} \\
\hline & $\mathbf{R}_{1075}=\mathbf{A}_{1075} / \mathbf{A}_{1375}$ & $\mathbf{R}_{1035}=\mathbf{A}_{1035} / \mathbf{A}_{1375}$ & \\
\hline 0 phr (Virgin NR) & 0.31 & 0.28 & $0.00 \%$ \\
\hline $10 \mathrm{phr}$ & 0.41 & 0.31 & $0.11 \%$ \\
\hline $20 \mathrm{phr}$ & 0.53 & 0.41 & $0.22 \%$ \\
\hline
\end{tabular}

Table 7.5 Grafting efficiency of VP Si-363 grafted onto NR molecules.

\begin{tabular}{cccc}
\hline $\begin{array}{c}\text { Amount of VP Si- } \\
\mathbf{3 6 3} \text { (phr) }\end{array}$ & $\begin{array}{c}\text { Amount of VP Si- } \\
\mathbf{3 6 3} \text { used (wt\%) }\end{array}$ & $\begin{array}{c}\text { Amount of grafted } \\
\text { VP Si-363 (wt\%) }\end{array}$ & $\begin{array}{c}\text { Grafting efficiency } \\
\text { (\%) }\end{array}$ \\
\hline 10 & 9.09 & 1.57 & 17.25 \\
20 & 16.67 & 3.10 & 18.60 \\
\hline
\end{tabular}

The ${ }^{1} \mathrm{H}-\mathrm{NMR}$ spectra of the purified VP Si-363-grafted NRs in Figure 7.11 show the characteristic peak of silane at the chemical shift at $3.6 \mathrm{ppm}$ assigned to the methylene proton of the alkoxy-group and the ethylene oxide repeating unit in the silane structure. The peak intensity increases with rising silane content indicating the increase of grafted silane on the NR chains. By taking the integrated peak area of the $-\mathrm{Si}-\mathrm{O}-\mathrm{CH}_{2}-\mathrm{CH}_{3}$ 
and $-\mathrm{Si}-\mathrm{O}-\left(\mathrm{C}_{2} \underline{\mathrm{H}}_{4} \mathrm{O}\right)_{5}-\mathrm{C}_{2}-\mathrm{C}_{12} \mathrm{H}_{25}$ of the grafted silane in the range of $4.0-3.5 \mathrm{ppm}$ and of

$-\stackrel{\mathrm{I}_{3}}{\mathrm{C}}=\mathrm{CH}$ - of $\mathrm{NR}$ at $5.1 \mathrm{ppm}$, the mole\% and wt\% of VP Si-363 grafted on NR were calculated according to Equations (7.3) and (7.5), and the results are shown in Tables 7.4 and 7.5, respectively. With 10 and 20 phr of VP Si-363 used for the reaction, grafted silanes of 0.11 and 0.22 mol\% or 1.57 and 3.10 wt\% on the NR molecules are obtained giving grafting efficiencies of 17.25 and $18.60 \%$, respectively.

The SEM-EDX technique was additionally used to confirm the presence of Siatoms in the grafted rubber and the analysis results of the purified samples are shown in Table 7.6.

Table 7.6 Chemical elements of VP Si-363-grafted NRs from SEM-EDX.

\begin{tabular}{lccc}
\hline \multirow{2}{*}{ Sample } & \multicolumn{3}{c}{ Chemical elements (wt\%) } \\
\cline { 2 - 4 } & $\mathbf{C}$ & $\mathbf{O}$ & $\mathbf{S i}$ \\
\hline Virgin NR & 98.2 & 1.8 & - \\
NR grafted with VP Si-363 $10 \mathrm{phr}$ & 94.1 & 5.8 & 0.1 \\
NR grafted with VP Si-363 $20 \mathrm{phr}$ & 88.6 & 11.2 & 0.2 \\
\hline
\end{tabular}

The elemental analysis data of the purified VP Si-363-grafted NRs reveals an increase of oxygen and silicon contents with increasing silane concentrations used in the reaction as shown in Table 7.6. Virgin NR contains no Si-atoms and a small amount of oxygen that is naturally present in the natural rubber. The detectable Si-component in the NR grafted with VP Si-363 confirms the presence of the silane fragment bonded to NR after all unreacted silane was removed in the purification step.

Under the grafting conditions, the peroxide initiator is decomposed giving the reactive radicals as shown in Scheme 7.1, and a possible reaction mechanism of the VP Si-363 grafting onto NR molecules in the presence of radicals is shown in Scheme 7.2.<smiles>CC1CC(C)(C)CC(OOC(C)(C)C)(OOC(C)(C)C)C1</smiles>$$
2 \mathrm{CH}_{3}-\stackrel{\mathrm{O}}{\mathrm{C}}-\mathrm{CH}_{3}+2 \cdot \mathrm{CH}_{3} \text { (II) }
$$

Scheme 7.1 Decomposition of 1,1'-di(tert-butylperoxy)-3,3,5-trimethylcyclohexane (Luperox® 231XL40).
} 


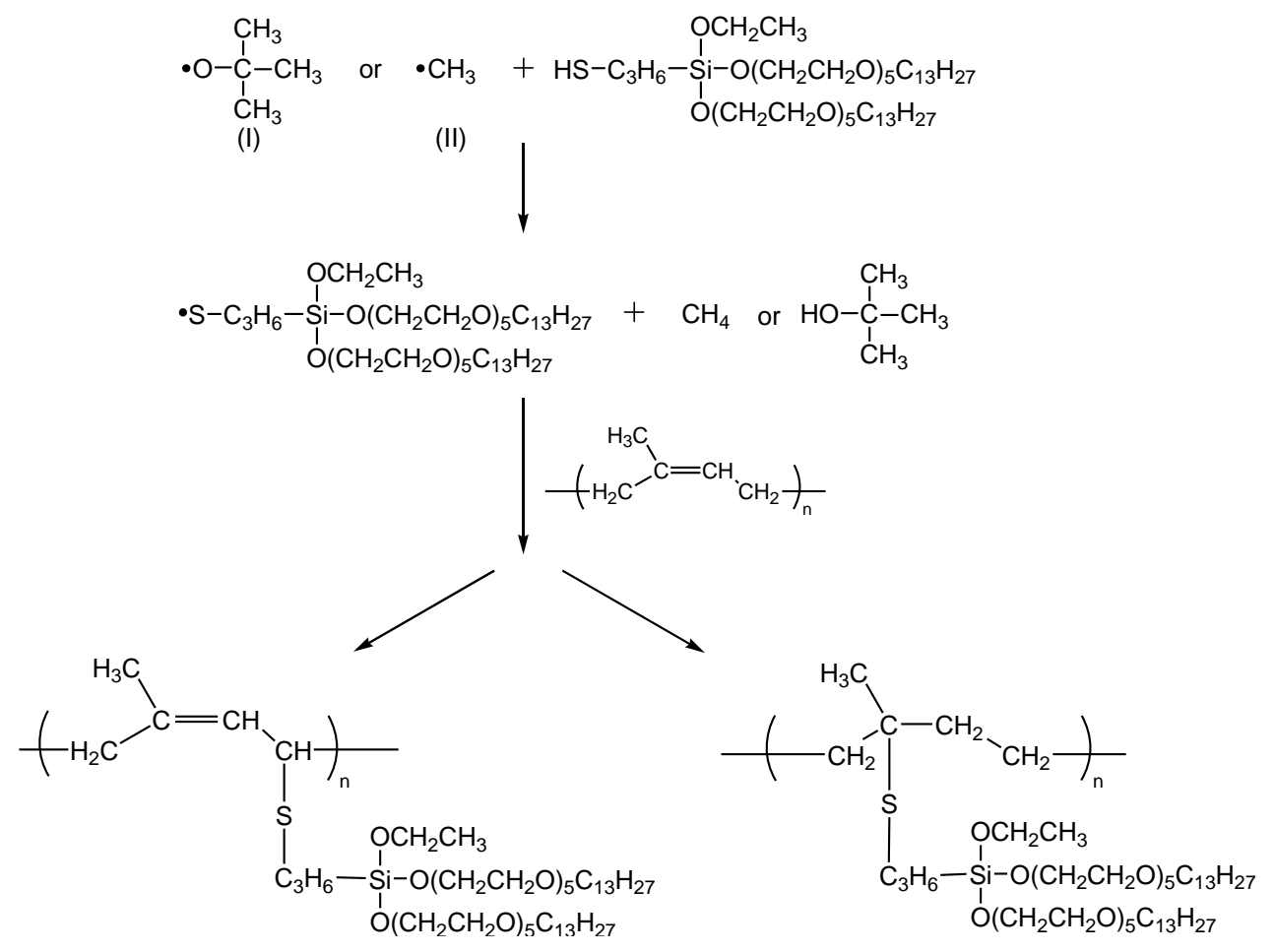

Scheme 7.2 Postulated reaction mechanism of VP Si-363 grafting onto NR molecules in the presence of radical species.

The Luperox® 231 XL40 is a diperoxide initiator that can be thermally decomposed to give two free radicals or radical species I $\left(\bullet \mathrm{OC}\left(\mathrm{CH}_{3}\right)_{3}\right)$ per molecule. The tert-butoxy radicals can further spit to create more reactive methyl radicals $\left(\bullet \mathrm{CH}_{3}\right)$ under the high grafting temperature, as shown in Scheme 7.1. It is probable that both types of free radicals formed in the system ${ }^{[32]}$, can initiate the grafting reaction on the NR which may proceed through two different pathways via $\mathrm{H}$-abstraction and addition ${ }^{[33]}$, as shown in Scheme 7.2. The reactive initiator transfers its radical to the sulfur on the coupling agent which subsequently attacks a double bond of the polymer molecule. The grafting reaction tends to proceed then through an abstraction reaction at the allylic position of the NR structure, more than through the addition reaction like with common sulfur vulcanization. ${ }^{[34]}$ Upon successful grafting a covalent bond C-S-C bond between rubber and silane is generated with the pendant alkoxy groups. 


\subsubsection{NXT-grafted NR}

The FTIR spectra of the purified NR grafted in presence of 10 and 20 phr of NXT are shown in comparison with that of virgin NR in Figure 7.12.

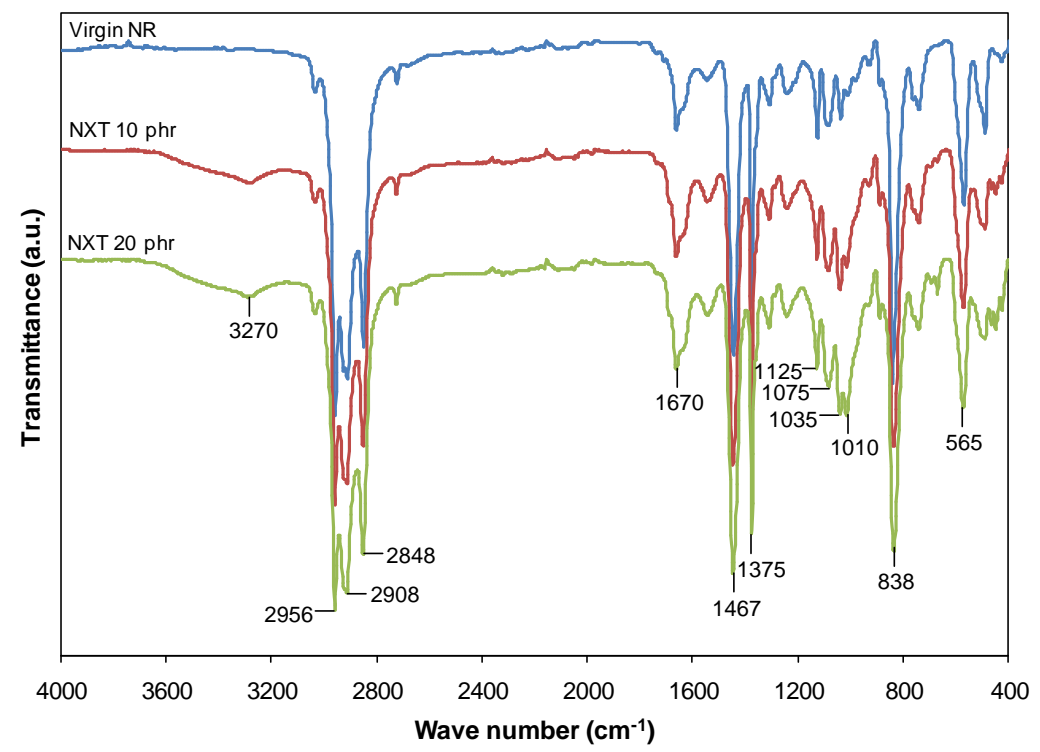

Figure 7.12 ATR-FTIR spectra of virgin NR and NR grafted with NXT at 10 and 20 phr.

The grafting of NXT silane onto the NR molecules was performed using the same conditions as applied to prepare VP Si-363-grafted NR. The blocked mercapto-group of NXT should be more thermally stable than -SH of VP Si-363, but because the steric effects are also different in NXT and VP Si-363, the reactivity towards the grafting reaction cannot be simply compared. Once the mercapto function is activated, the NXT with three ethoxy groups should be more reactive than VP Si-363 with two bulky long alkoxy-groups. The FTIR spectra in Figure 7.12 clearly show absorption bands at $3270 \mathrm{~cm}^{-1}$ due to $\mathrm{O}-\mathrm{H}$ stretching, and at 1075 and $1035 \mathrm{~cm}^{-1}$ assigned to Si-O-C and Si-O-Si deformations, respectively, as previously observed in the spectra of the VP Si-363-grafted NR in Figure 7.10. For NR-g-NXT, there is an additional strong band at $1010 \mathrm{~cm}^{-1}$ which may be assigned to the $\mathrm{Si}-\mathrm{OH}$ group ${ }^{[35]}$ due to hydrolysis of the ethoxy groups. Compared with the spectra of NR-g-VP Si-363 in Figure 7.10, the strong increase of peak intensity at 1035 and $1010 \mathrm{~cm}^{-1}$ may be attributed to the faster reaction of the tri-ethoxy groups of NXT compared to the two bulky alkoxy groups with only one ethoxy group of VP Si-363. The hydrolysis of ethoxy groups by moisture during the grafting reaction leads to hydroxyl 
groups $\mathrm{Si}-\mathrm{OH}$ which can further form a crosslink $\mathrm{Si}-\mathrm{O}-\mathrm{Si}$ in the grafted rubber. By taking the peak height at 1075 and $1035 \mathrm{~cm}^{-1}$ relative to the peak at $1375 \mathrm{~cm}^{-1}$ as internal standard, the increased amount of the silane used for the grafting reaction with NR results in the increase of peak height ratios or $R$ values as shown in Table 7.7, indicating the presence of more grafted silane fragments.

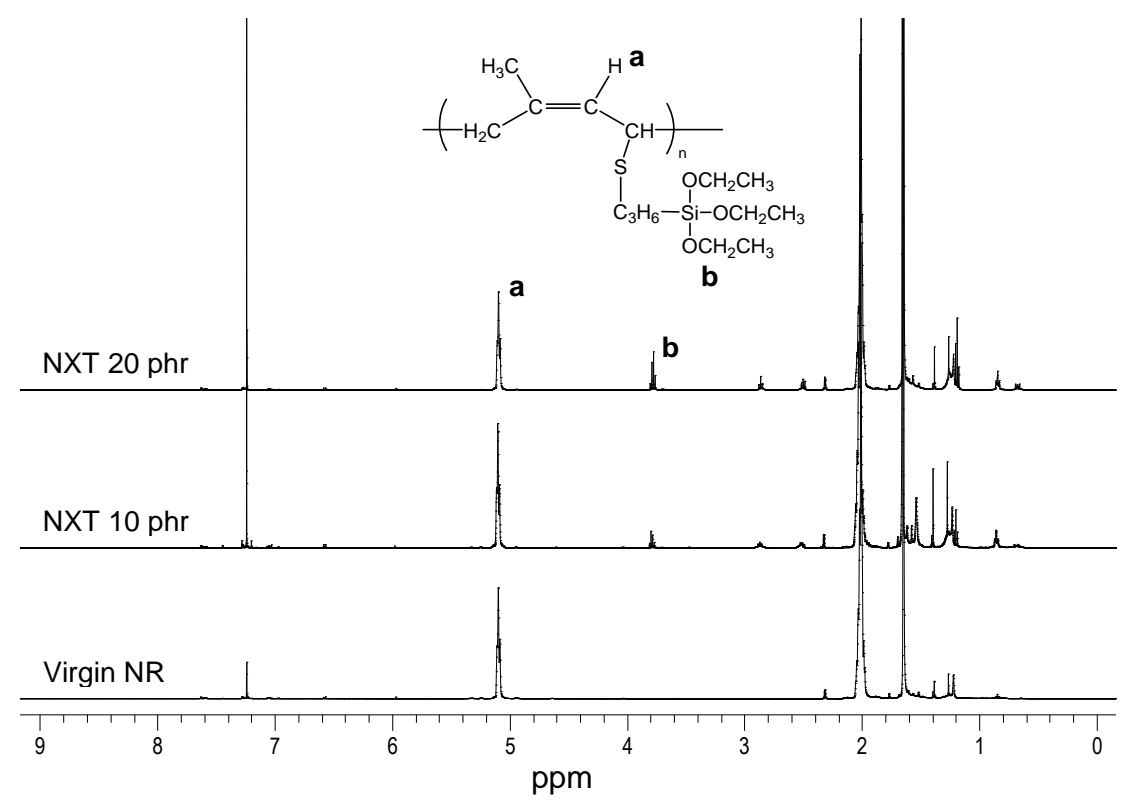

Figure $7.13{ }^{1} \mathrm{H}$-NMR spectra of virgin NR and NR grafted with NXT at 10 and 20 phr.

Table 7.7 The extent of NXT grafting (R) onto NR molecules from ATR-FTIR and mol\% of NXT on NR molecules from ${ }^{1} \mathrm{H}$-NMR.

\begin{tabular}{|c|c|c|c|}
\hline \multirow{2}{*}{$\begin{array}{c}\text { Amount of NXT } \\
\text { (phr) }\end{array}$} & \multicolumn{2}{|c|}{ R value from ATR-FTIR } & \multirow{2}{*}{$\begin{array}{l}\text { Mol\% of NXT } \\
\text { from }{ }^{1} \mathrm{H} \text { NMR }\end{array}$} \\
\hline & $\mathbf{R}_{1075}=\mathbf{A}_{1075} / \mathbf{A}_{1375}$ & $R_{1035}=A_{1035} / A_{1375}$ & \\
\hline 0 phr (Virgin NR) & 0.31 & 0.22 & $0.00 \%$ \\
\hline $10 \mathrm{phr}$ & 0.49 & 0.42 & $0.66 \%$ \\
\hline $20 \mathrm{phr}$ & 0.55 & 0.49 & $1.32 \%$ \\
\hline
\end{tabular}

Table 7.8 Grafting efficiency of NXT grafted onto NR molecules.

\begin{tabular}{cccc}
\hline $\begin{array}{c}\text { Amount of NXT } \\
\text { (phr) }\end{array}$ & $\begin{array}{c}\text { Amount of NXT } \\
\text { used (wt\%) }\end{array}$ & $\begin{array}{c}\text { Amount of grafted } \\
\text { NXT (wt\%) }\end{array}$ & $\begin{array}{c}\text { Grafting efficiency } \\
(\%)\end{array}$ \\
\hline 10 & 9.09 & 3.43 & 37.69 \\
20 & 16.67 & 6.68 & 40.07 \\
\hline
\end{tabular}


Figure 7.13 shows ${ }^{1} \mathrm{H}-\mathrm{NMR}$ spectra of the purified NXT-grafted NRs prepared by using two different NXT amounts of 10 and 20 phr in comparison with that of virgin NR. The grafted rubber shows a characteristic peak of NXT-silane at a chemical shift of 3.8 ppm assigned to the proton of the ethoxy-groups of the silane. This peak is intensified with increasing silane content and the mol\% of NXT-silane in the grafted NR as calculated according to Equation (7.4) is shown in Table 7.7. The grafted silane on the NR molecules increases with increasing amount of silane used for the reaction, and the mol\% as well as the grafting efficiency of NXT grafted on NR are twice that of VP Si-363 grafted on NR (Table 7.8 vs. 7.5). Even thought the blocked mercapto-group of NXT may be more difficult to activate than the $-\mathrm{SH}$ group of VP Si-363, once the $-\mathrm{S} \bullet$ is formed the higher steric effect of the long alkoxy-groups in the VP Si-363 molecule may lead to more difficulties to react with the NR molecules. The lower steric effect of the ethoxy-groups of NXT makes it easier for the grafting reaction but may also lead to a loss of ethoxy methylene-protons via hydrolysis and self-condensation, resulting in $\mathrm{Si}-\mathrm{OH}$ and $\mathrm{Si}-\mathrm{O}-\mathrm{Si}$ bonds, as seen by the strong increase of the FTIR peak intensities at 1035 and $1010 \mathrm{~cm}^{-1}$ in Figure 7.12.

The presence of the silane grafted on the NR molecules is confirmed by elemental analysis, as reported in Table 7.9. The amounts of silane used for the grafting reaction were at 10 and $20 \mathrm{phr}$, and the materials were purified to eliminate any unreacted silane prior to analysis.

Table 7.9 Chemical elements of NXT-grafted NRs from SEM-EDX.

\begin{tabular}{lccc}
\hline \multicolumn{1}{c}{ Sample } & \multicolumn{3}{c}{ Chemical elements (wt\%) } \\
\cline { 2 - 4 } & $\mathbf{C}$ & $\mathbf{O}$ & $\mathbf{S i}$ \\
\hline Virgin NR & 98.2 & 1.8 & - \\
NR grafted with NXT 10 phr & 97.5 & 2.4 & 0.1 \\
NR grafted with NXT 20 phr & 96.8 & 3.0 & 0.2 \\
\hline
\end{tabular}

The grafting of silane fragments on the NR molecules is proved by the presence of silicon atoms in the modified NR, and the increase of silane content used for the reaction results in increase of $\mathrm{Si}$ and $\mathrm{O}$ components in the grafted materials. When compared to the NR-g-VP Si-363 elemental analysis results in Table 7.6, the NR-g-NXT shows the same Si-contents but lower $\mathrm{O}$ contents in accordance with their molecular structures. The equal Si- contents could be due to experimental uncertainties at these low levels. 


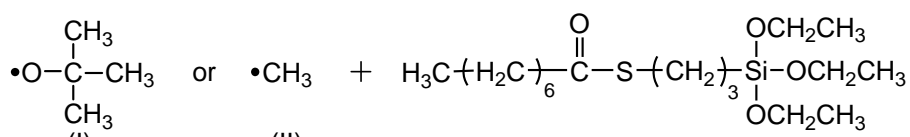

(I)

(II)

$$
\downarrow
$$

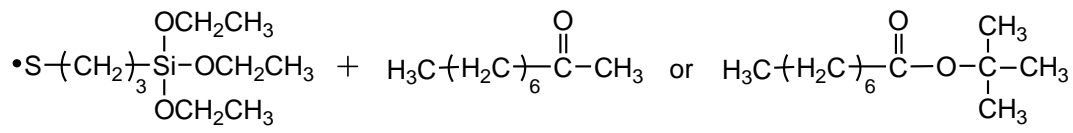

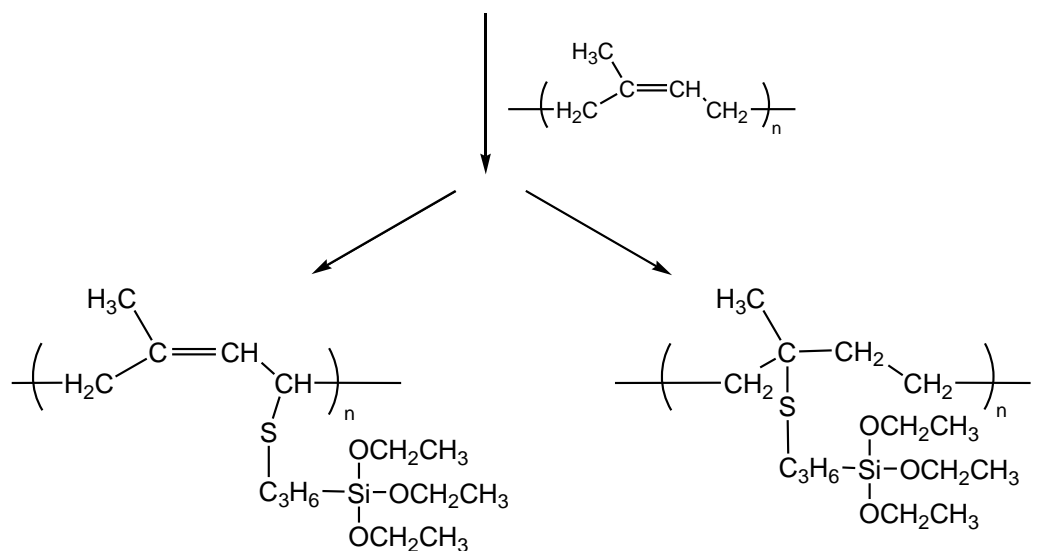

Scheme 7.3 Postulated reaction mechanism of NXT grafting onto NR molecules in the presence of radical species.

The possible reaction mechanism of NXT-grafting of NR as displayed in Scheme 7.3 involves two reaction pathways: abstraction and addition, like the reaction mechanisms proposed for the grafting of VP Si-363 onto NR molecules in Scheme 7.2. After the grafting reaction, the deblocked carboxylic fragment of NXT is left in the grafted rubber as by-product either in the form of an aldehyde or carboxylic acid. The presence of this by-product is not visible in the FTIR and ${ }^{1} \mathrm{H}$-NMR spectra because the materials were purified prior to the analysis.

\subsection{Conclusions}

Silane-grafted natural rubber is successfully prepared under melt mixing conditions in an internal mixer at $140^{\circ} \mathrm{C}$ using $0.1 \mathrm{phr}$ of $1,1^{\prime}$-di(tert-butylperoxy)-3,3,5trimethylcyclohexane (Luperox® 231XL40) as initiator. The NXT-grafting and VP Si-363grafting of NR are comfirmed by elemental analysis via the SEM-EDX technique in which the modified NRs have silicon ( $\mathrm{Si}$ ) atoms in their structures, and the amounts of oxygen and silicon in the grafted NRs increase with increasing amount of silane used for the 
reaction. Both VP Si-363-grafted NR and NXT-grafted NR show infrared absorption bands at 3270,1075 and $1035 \mathrm{~cm}^{-1}$ which can be assigned to the deformations of $-\mathrm{OH}$, Si-O-C and $\mathrm{Si}-\mathrm{O}-\mathrm{Si}$, respectively, and the ${ }^{1} \mathrm{H}-\mathrm{NMR}$ absorption band at 3.8-3.6 ppm assigned to the methylene protons of alkoxy groups. By calculation using the integrated peak areas of $-\mathrm{OCH}_{2}$ - of alkoxy-groups of silane and $=\mathrm{CH}$ of $\mathrm{NR}$, the use of 10 and 20 phr of silane results in grafted VP Si-363 on NR of 0.11 and 0.22 mol\% or grafting efficiency of $\approx 18 \%$, and grafted NXT of 0.66 and 1.32 mol\% or grafting efficiency of $\approx 38 \%$, respectively. The differences in grafting efficiency may tentatively be related to the respective molecular structures of the silanes.

\subsection{References}

1. L.T. Zhuravlev. Colloids Surf. A. 173, 1 (2000).

2. A. Hunsche, U. Görl, H.G. Koban, T. Lehmann, Kautsch. Gummi Kunstst., 51, 525 (1998).

3. L.A.E.M. Reuvekamp, J.W. ten Brinke, P.J. van Swaaij, J.W.M. Noordermeer, Rubber Chem. Technol., 75, 187 (2002).

4. W. Kaewsakul, K. Sahakaro, W.K. Dierkes, J.W.M. Noordermeer, Rubber Chem. Technol., 85, 277 (2012).

5. J.W. ten Brinke, S.C. Debnath, L.A.E.M. Reuvekamp, J.W.M. Noordermeer, Comp. Sci. Technol., 63, 1165 (2003).

6. J.W. ten Brinke, P.J. van Swaaij, L.A.E.M. Reuvekamp, J.W.M. Noordermeer, Kautsch. Gummi Kunstst., 55, 244 (2002).

7. T. Jesionowski, A. Krysztafkiewicz, Appl. Surf. Sci., 172, 18 (2001).

8. C.H. Lee, S.H. Park, W. Chung, J.Y. Kim, S.H. Kim, Colloids Surf. A: Physicochem. Eng. Aspects, 384, 318 (2011).

9. B. Kitiyanan, J.H. O'Haver, J.H. Harwell, S. Osuwan, Langmuir, 12, 2162 (1996).

10. W.H. Waddell, J.H. O'Haver, L.R. Evans, J.H. Harwell, J. Appl. Polym. Sci., 55, 1627 (1995).

11. V. Thammathadanukul, J.H. O'Haver, J.H Harwell, S. Osuwan, N. Na-ranong, W.H. Waddell, J. Appl. Polym. Sci., 59, 1741 (1996).

12. M. Tiwari, "Plasma Coating of Silica: A Key to Improved Dispersion and Properties of Reinforced Elastomer Blends", Ph.D. Thesis: 2010, Dept. of Elastomer Technology and Engineering, Univ. of Twente, Enschede, the Netherlands.

13. G. Mathew, M.-Y. Huh, J.M. Rhee, M.-H. Lee, C. Nah, Polym. Adv. Technol., 15, 400 (2004). 
14. C. Nah, M.-Y. Huh, J.M. Rhee, T.-H. Yoon, Polym. Int., 51, 510 (2002).

15. M. Tiwari, J.W.M. Noordermeer, W.J.V. Ooij, W.K. Dierkes, Polym. Adv. Technol., 19, 1672 (2008).

16. S. Jain, J.G.P. Goossens, M. Van Duin, Macromol. Symp., 233, 225 (2006).

17. G. Colucci, A.D. Gianni, R. Bongiovanni, A. Priola, F. Torello, S. Bracco, P. Sozzani, Macromol. Symp., 296, 550 (2010).

18. A. Bertora, M. Castellano, E. Marsano, A. Turturro, M. Alessi, L. Conzatti, P. Stagnaro, G. Colucci, A. Priola, Macromol. Mater. Eng., 296, 455 (2011).

19. Y.-T. Shieh, T.-H. Tsai, J. Appl. Polym. Sci., 69, 255 (1998).

20. K. Nakashima, P.H. Solomon, Infrared Absorption Spectroscopy, $2^{\text {nd }}$ Edition, Holdenday, San Francisco (1997).

21. J. Lin, J.A. Siddiqui, R.M. Ottenbrite. Polym. Adv. Technol., 12, 285 (2001).

22. K. Sirisinha, S. Chimdist, Polym. Test., 25, 518 (2006).

23. C. Jiao, Z. Wang, Z. Gui, Y. Hu, Eur. Polym. J., 41, 1204 (2005).

24. M.-C. Brochier Salon, P.-A. Mayle, M. Abdelmouleh, S. Boufi, M. N. Belgacem. Colloid Surface A., 312, 83 (2008).

25. T. Ogasawara, A. Yoshino, H. Okabayashi., C. J. O'Connor. Colloid Surface A., 180, 317 (2001).

26. F. Schapman, J.P. Couvercelle, C. Bunel, Polymer, 39, 4955 (1998).

27. C. Yin, Q. Zhang, J. Gu, J. Zheng, G. Gong, T. Liang, H. Zhang, J. Appl. Polym. Sci., 128, 2262 (2013).

28. K. Sirisinha, M. Boonkongkaew, S. Kositchaiyong, Polym. Test., 29, 958 (2010).

29. M. Beltrán, C. Mijangos, Polym. Eng. Sci., 40, 1534 (2000).

30. S. Lenka, P.L. Nayak, I.B. Mohanty, S.N. Mishra, J. Appl. Polym. Sci., 30, 2711 (1985).

31. L. Thiraphattaraphun, S. Kiatkamjornwong P. Prasassarakich, S. Damronglerd, J. Appl. Polym. Sci., 81, 428 (2001).

32. S.S. Pesetskii, B. Jurkowski, Y.M. Krivoguz, K. Kelar, Polymer, 42, 469 (2001).

33. R.S. Lehrle, S.L. Willist, Polymer, 38, 5937 (1997).

34. P.C. de Oliveira, A.M. de Oliveira, A. Garcia, J. C. de Souza Barboza, C.A. de Carvalha Zavaglia, A.M. dos Santos, Eur. Polym. J., 41, 1883 (2005).

35. M. Abdelmouleh, S. Boufi, M.N. Belgacem, A. Dufresne, Compos. Sci. Technol., 67, 1627 (2007). 


\section{Silane-Grafted-Natural Rubber as Compatibilizer in Silica- Reinforced Natural Rubber}

The effect of silane-grafted-natural rubber (NR) as compatibilizer in silica-filled NR compounds is studied by using NXT- and VP Si-363-grafted-NRs prepared by using 10 and 20 phr of the silanes. The silane-grafted-NRs were used in a range of 5-20 phr, so as to give 0.8-6.1 wt\% silane functionality relative to the silica. The properties of the silicafilled NR compounds compatibilized with the silane-grafted-NRs are studied in comparison with those compatibilized by straight use of a silane TESPT at varying amounts in the same range, and with 8.6 wt\% relative to the silica. The use of the silane-grafted-NRs as compatibilizers decreases the Mooney viscosity, Payne effect and silica flocculation, improves filler-rubber interaction, and significantly increases the tensile modulus, reinforcement index and tensile strength of the silica-filled NR-compounds compared to the non-compatibilized one. At the same silane-content, the use of silane-grafted-NRs gives slightly better properties than the straight use of the same silane, and the use of $N X T$-grafted-NR as compatibilizer shows a better improvement of overall properties than the use of VP Si-363-grafted NR. In the presence of silane-grafted-NRs as compatibilizers, the glass transition temperatures $\left(T_{g}\right)$ of the silica-filled $N R$ vulcanizates slightly shift to a higher temperature, while the tan $\delta$ values at $5^{\circ} \mathrm{C}$ remain more or less the same and the tan $\delta$ values at $60^{\circ} \mathrm{C}$ decrease compared to that with TESPT and without compatibilizer. 


\subsection{Introduction}

Chemical modification of natural rubber (NR) by epoxidation ${ }^{[1]}$ has been widely used to increase the polarity, improve some properties of the rubber and enhance the compatibility with polar substances including silica. The silanol-groups on the silica surface interact with the epoxide groups of ENR through hydrogen bonds during processing and ring opening of the epoxide groups can form chemical bonds with silica via a condensation reaction with the silanol groups. ${ }^{[2,3]}$ Silica-filled epoxidized polybutadiene (EpBR) and epoxidized styrene-butadiene rubber (EpSBR) show improvement in the chemical interaction between the rubbers and silica as well as improved filler dispersion. ${ }^{[4,5]}$ A restriction on polymer chain movement by presence of the chemical bonds between the epoxide-groups and silica resulted in a shift of the glass transition temperature $\left(T_{g}\right)$ of the silica-filled epoxidized rubber. ${ }^{[4-6]}$

An alternative pathway to enhance the compatibility between polymer and silica is to use silane modified polymers instead of straight use of a silane. Grafting of silane coupling agents onto polymer molecules has been reported by using different types of silanes such as vinyltrimethoxysilane $(\mathrm{VTMS})^{[7-9]}$, vinyltriethoxysilane $(\mathrm{VTES})^{\left[{ }^{[-12]}\right.}$ and mercaptopropyl-trimethoxysilane. ${ }^{[12-13]}$ Vinylsilanes such as VTMS and VTES are widely used to modify polyolefins to improve the strength properties of the pure polymers via a self-crosslinking reaction. The grafting of vinylsilanes onto the polyolefins was carried out in melt condition using dicumyl peroxide $(\mathrm{DCP})$ as initiator ${ }^{[7-10]}$, and under inert conditions at low temperature with azobisisobutyronitrile (AIBN) as initiator. ${ }^{[11]}$ Increased crosslink density in the silane-grafted polyolefins leads to improvement in thermal stability and tensile properties. ${ }^{[8,9]}$ Polypropylene (PP) grafted with VTES and PP-graft-VTES/silica nanocomposites were investigated in which the grafted silane affects the crystallization temperature of PP and the impact strength of the composites. ${ }^{[11]}$ VTES was grafted onto styrene-butadiene rubber at a temperature of about $60-70^{\circ} \mathrm{C}$ by using potassium persulfate $(\mathrm{KPS})^{[12]}$ or benzoyl peroxide $(\mathrm{BPO})^{[13]}$ as initiators under nitrogen atmosphere. The use of SBR-g-VTES for a silica-filled compound led to improved cure and tensile properties, ${ }^{[12,13]}$ and a shift of the glass transition temperature ${ }^{[12]}\left(T_{g}\right)$ to higher temperature due to the good interactions between silica and SBR-g-VTES, that restrict movement of the SBR-chains. Further, liquid polybutadiene (PB) has been modified with mercaptopropyltrimethoxysilane and mercaptopropyltriethoxysilane to improve the reinforcing efficiency of silica in this rubber. ${ }^{[14-15]}$ The mercapto-silane-modified PB was prepared in solution at $75^{\circ} \mathrm{C}$ under inert atmosphere with AIBN as initiator, and the grafting reaction proceeded through radical addition of the thiol group of the silane to the 
double bond of PB, introducing alkoxy groups on the rubber chains. After a silanization reaction of silica with the silane-grafted $\mathrm{PB}$, the silica characteristics showed a more hydrophobic surface, which was proved by an increase of contact angle ${ }^{[14]}$ and a decrease of hydroxyl groups on the silica surface. ${ }^{[15]}$ The covalent bond Si-O-C of the silanized PB was analyzed by proton-, carbon- and silicon nuclear magnetic resonance spectroscopy $\left({ }^{1} \mathrm{H}-\mathrm{NMR},{ }^{13} \mathrm{C}-\mathrm{NMR}\right.$ and $\left.{ }^{29} \mathrm{Si}-\mathrm{NMR}\right) .{ }^{[14,16]}$ The better interaction of silica and rubber via silane-grafted PB improved the silica dispersion and tensile strength. ${ }^{[15]}$ Therefore, the use of silane-modified polymer chains is an alternative method to improve bonding between hydrophilic silica and hydrophobic hydrocarbon rubber.

This chapter reports the influence of NXT-grafted-NRs and VP Si-363-graftedNRs, i.e. NR-g-NXT and NR-g-VP Si-363, respectively, as compatibilizers in silica-filled NR. The non-purified silane-grafted-NRs were used in a range of 5-20 phr and the respective amounts of silanes were also straightly used in another set of compounds, for comparison. The properties of both compounds and vulcanizates compatibilized with silane and silane-grafted-NR are discussed in comparison with those without compatibilizer and with reference TESPT.

\subsection{Experimental}

\subsubsection{Materials and compound preparation}

The compounding ingredients, i.e. NR (RSS3), TESPT, silica (Zeosil 1165MP), TDAE oil, ZnO, TMQ, stearic acid, DPG, CBS and sulfur, used for preparation of the silicafilled NR compounds in this chapter were as described in Chapter 3. NXT and VP Si-363 are as detailed in Chapter 5 and the information shown in Table 5.1. The NXT-grafted-NR and VP Si-363-grafted-NR were prepared according to the methods described in Chapter 7.

The NXT- and VP Si-363-grafted-NRs, pre-prepared by using 10 and 20 phr of silane, were used without purification in order to simplify the process. The amounts of grafted-NXT and grafted-VP Si-363 on the NR as well as the grafting efficiencies are as previously shown in Tables 7.8 and 7.5. To study the effect of NXT- and VP Si-363grafted-NRs as compatibilizers in silica-filled NR, the silane-grafted-NRs were varied at 5, 10,15 and 20 phr as part of the rubber matrix. Total silane contents based on calculation, either in the form of free silane and of grafted silane in the compatibilizers are summarized in Table 8.1. 
Table 8.1 Total NXT and VP Si-363 contents including both reacted and unreacted parts in different amounts of NXT- and VP Si-363-grafted-NR being used as compatibilizers in the silica-filled NR compounds.

\begin{tabular}{cc}
\hline Amount of NXT- and VP Si-363-grafted-NR & $\begin{array}{c}\text { Amount of total silane } \\
\text { (wt\% rel. to silica) }\end{array}$ \\
\hline Silane-grafted-NR with 10 phr of silane \\
- $5 \mathrm{phr}$ & 0.8 \\
- $10 \mathrm{phr}$ & 1.7 \\
- $15 \mathrm{phr}$ & 2.5 \\
\hline Silane-grafted-NR with 20 phr of silane & 3.4 \\
- $\quad 5 \mathrm{phr}$ & \\
- $\quad 10 \mathrm{phr}$ & 1.5 \\
- $\quad 20 \mathrm{phr}$ & 3.0 \\
\hline
\end{tabular}

The silica-filled NR compound formulations with the various amounts of silanegrafted-NRs and the straight silanes are shown in Table 8.2 together with the references. The properties of the silica-filled NR compounds with the silane-grafted-NRs as compatibilizers were studied in comparison with the results of silica-filled NR with straight silanes at different amounts, as calculated based on the silane contents used in the preparation of the silane-grafted rubber, as shown in Table 8.1. The mixing procedures are given in Table 8.3. The mixer type and temperature setting are as described in Chapter 5. 
Table 8.2 Compound formulations.

\begin{tabular}{lcc|cc|cc}
\hline \multirow{2}{*}{ Ingredients } & \multicolumn{6}{c}{ Parts per hundred parts of rubber (phr) } \\
\cline { 2 - 7 } & \multicolumn{2}{c}{ References } & \multicolumn{2}{c}{ Silane-grafted-NRs } & Straight use of silane \\
\hline RSS3 & 100.0 & 100.0 & $95.0-80.0$ & $95.0-80.0$ & 100.0 & 100.0 \\
TESPT & - & $4.7^{*}$ & - & - & - & - \\
NXT-grafted-NR** & - & - & $5.0-20.0$ & - & - & - \\
VP Si-363-grafted-NR** & - & - & - & $5.0-20.0$ & - & - \\
NXT & - & - & - & - & $0.8-6.1^{\star \star *}$ & - \\
VP Si-363 & - & - & - & - & - & $0.8-6.1^{\star * *}$ \\
Zeosil 1165MP & 55.0 & 55.0 & 55.0 & 55.0 & 55.0 & 55.0 \\
TDAE oil & 8.0 & 8.0 & 8.0 & 8.0 & 8.0 & 8.0 \\
ZnO & 3.0 & 3.0 & 3.0 & 3.0 & 3.0 & 3.0 \\
TMQ & 1.0 & 1.0 & 1.0 & 1.0 & 1.0 & 1.0 \\
Stearic acid & 1.0 & 1.0 & 1.0 & 1.0 & 1.0 & 1.0 \\
DPG & 1.0 & 1.0 & 1.0 & 1.0 & 1.0 & 1.0 \\
CBS & 1.5 & 1.5 & 1.5 & 1.5 & 1.5 & 1.5 \\
Sulfur & 1.5 & 1.5 & 1.5 & 1.5 & 1.5 & 1.5 \\
\hline
\end{tabular}

Remarks: * TESPT 4.7 phr equals 8.6 wt\% rel. to silica; ${ }^{* *}$ NXT- and VP Si-363-grafted-NRs were prepared by using silane contents at 10 and $20 \mathrm{phr}$; ${ }^{* \star}$ Silane contents for straight use were calculated based on silane loadings in the non-purified silane-grafted-NR as shown in Table 8.1.

Table 8.3 Mixing procedures.

\begin{tabular}{lc}
\multicolumn{1}{c}{ Mixing procedures } & $\begin{array}{c}\text { Cumulative } \\
\text { time (mins.) }\end{array}$ \\
\hline Step 1 : Internal mixer & 2 \\
- NR and silane-grafted-NR (or NR only) mastication & 7 \\
- Addition of first half of silica (and $1 / 2$ of silane if any) & 12 \\
- Addition of second half of silica (and $1 / 2$ silane if any) and TDAE oil & 15 \\
\hline - Addition of ZnO, stearic acid and TMQ & 5 \\
\hline Step 2 : Two roll mill & 5 \\
\hline
\end{tabular}

\subsubsection{Property testing and characterization}

Mooney viscosity of the compounds after step 2 and cure characteristics were tested according to the methods as described in Chapter 5. Payne effect and flocculation rate constant of the final compounds (with curatives) were analyzed according to the methods as detailed in Chapter 3. Bound rubber content of the non-productive compounds after step 1 was analyzed following the procedures as described in Chapter 3.

The compounds were press-cured to their respective optimum cure times $\left(t_{c 90}\right)$ and tensile as well as dynamic mechanical properties were tested following the methods 
as described in Chapter 5. Tensile fractured surfaces were analyzed by using the scanning electron microscopy (SEM) technique, as described in Chapter 4.

The properties are reported as function of silane contents in the compounds compatibilized by both silane-grafted-NRs and with straight use of silane.

\subsection{Results and discussion}

8.3.1 Effect of NXT-grafted-NR as compatibilizer and use of straight NXT silane on the properties of silica-filled NR compounds

8.3.1.1 Mooney viscosity, bound rubber content, Payne effect and flocculation rate constant

For filled rubber compounds, the viscosity increment depends on several factors, to include mastication time ${ }^{[17]}$, mixing time ${ }^{[18]}$, filler types ${ }^{[19,20]}$, filler contents ${ }^{[20,21]}$, compatibilizer or coupling agent ${ }^{[22,23]}$ and storage time. ${ }^{[19,24]}$ The change of compound viscosity is strongly influenced by the levels of filler-filler and filler-rubber interactions which consequently have an influence on the final properties of the filled rubber. The decrease of compound viscosity results in a lower mixing torque and consequently less heat generation or lower compound temperature.
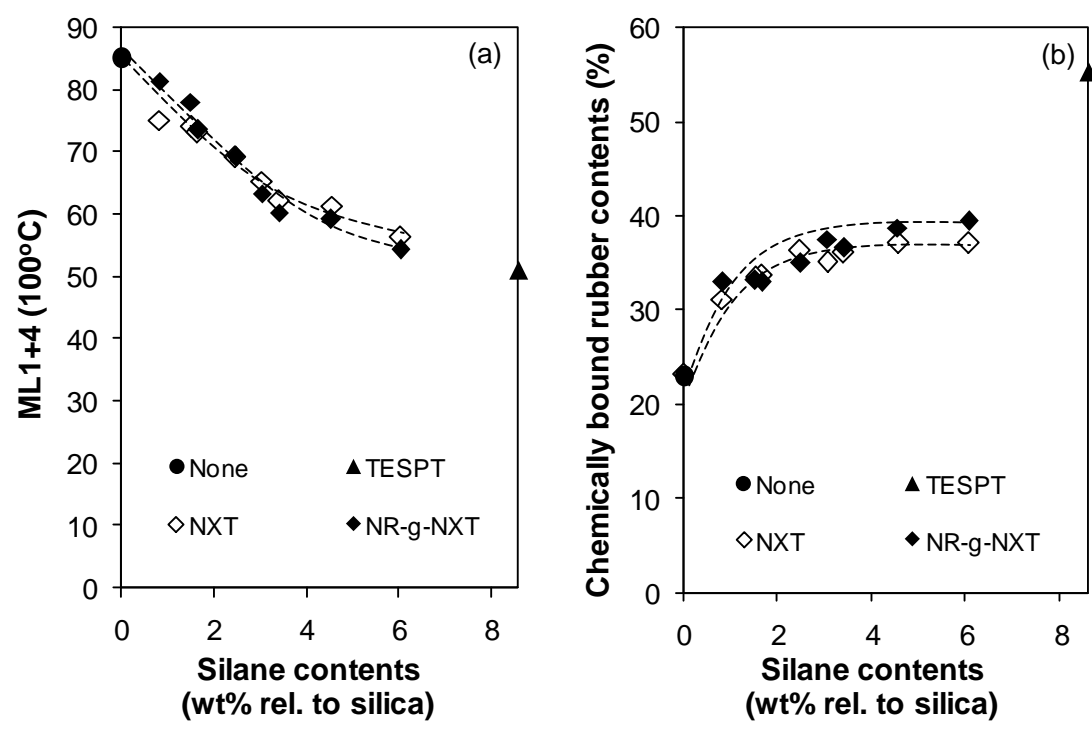

Figure 8.1 Mooney viscosity (a) and bound rubber contents (b) of silica-filled NR compounds with use of straight NXT (open symbols) and NXT-grafted-NR (closed symbols). 
Mooney viscosities of the silica-filled NR compounds decrease with increasing amounts of silane either in the form of NR-g-NXT or straight used NXT, and both sets of compounds show similar properties, as shown in Figure 8.1(a). The Mooney viscosity of the filled-NR with NXT and NXT-grafted-NR with 6 wt\% of silane relative to the silica, decreases to almost the same level as that of the reference compound with TESPT at 8.6 $w t \%$ relative to the silica. This level of $8.6 \mathrm{wt} \%$ of TESPT relative to the silica was found to be the optimum, as calculated based on the work described by Guy et al. ${ }^{[25]}$ For silicafilled compounds, the viscosity reduces when filler dispersion is improved. ${ }^{[23]}$ Thus, the incorporation of NXT or NXT-grafted-NR improves the silica dispersion and consequently reduces the Mooney viscosity due to a diminished hydrophilic character of the silica after the silanization reaction. As expected, the silica-filled NR compound without compatibilizer shows the highest Mooney viscosity because of the strong filler-filler interactions via hydrogen bonding of the silanol groups on the silica surfaces that lead to poor dispersion and large agglomerates to obstruct flow.

Figure 8.1 (b) shows the chemically bound rubber contents of silica-filled NR compounds with different compatibilizers in which the use of either NR-g-NXT or straight NXT significantly increases the bound rubber, i.e. increased silica-rubber interaction compared to the non-compatibilized one, but still to a significantly lower level compared with the reference compound with TESPT. The chemically bound rubber contents increase with rising amount of NXT silane up to $3.4 \mathrm{wt} \%$ relative to the silica, thereafter the values level off. The use of NXT-grafted-NR tends to show a marginally higher chemically bound rubber content than the straight use of NXT, in accordance with the Payne effect in Figure 8.2(a). The partially pre-bonded silane to NR may enhance the compatibility between rubber and silica and allow the ethoxy-groups of NXT to react more effectively with the silica during the silanization reaction. However, the use of TESPT in the silica-filled NR compound still gives an outstanding chemically bound rubber content, which is due to its tetrasulfide structure that results in pre-mature crosslinking with the NR molecules $^{[26]}$ and the bis-triethoxy-groups to react with the silanol groups during silanization. 

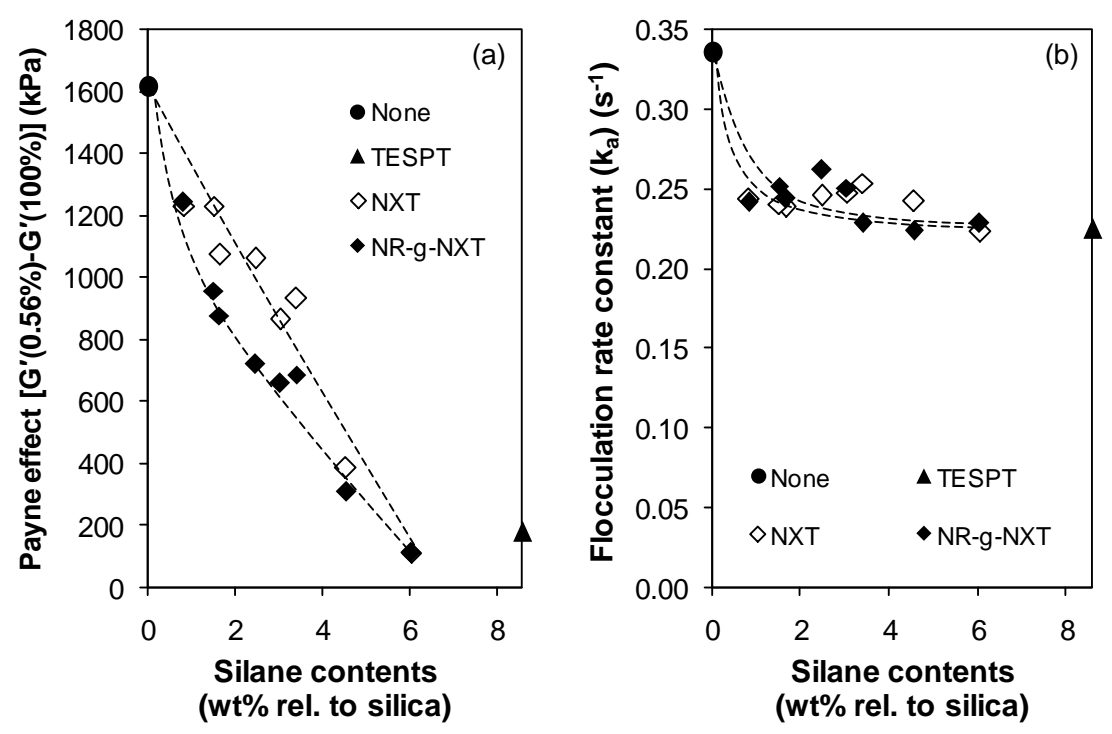

Figure 8.2 Payne effect (a) and flocculation rate constant (b) of silica-filled NR compounds with straight use of NXT (open symbols) and NXT-grafted-NR (closed symbols).

The filler-filler interaction or Payne effect of the silica-filled NR compounds with both NXT and NXT-grafted-NR is remarkably decreased with increasing silane content to finally meet the same level of Payne effect of the compound with TESPT, as shown in Figure 8.2(a). The ethoxy-groups of the NXT-silane undergo a silanization reaction with the silanol-groups of the silica and reduce the filler-filler interactions. Increasing amounts of NXT-silane further decrease the Payne effect of the compounds and the use of NXTgrafted-NR gives even slightly lower Payne effects than the straight use of NXT.

The flocculation behavior of the silica in the rubber matrix is due to the different polarity between silica and rubber causing re-agglomeration of silica aggregates or demixing, which will influence the properties of the material. ${ }^{[27]}$ The flocculation rate constant $\left(k_{a}\right)$ is used to determine the degree of re-agglomeration of the silica to form a stable silica-silica network under heat treatment. ${ }^{[28]}$ At higher temperatures, silica aggregates can migrate easier in the rubber matrix to reform agglomerates and re-create a filler-filler network. The silica-filled compound without silane shows a strong silica flocculation tendency as shown in Figure 8.2(b) due to hydrogen bonding between the silanol groups on the silica surface. The addition of both NXT and NXT-grafted-NR at only a small amount already reduces the flocculation rate of the silica-filled compounds, like in the case of TESPT, due to reduction of the silica polarity by silanization of the silane onto the silica 
surface. However, further increase of NXT-contents has no additional effect on the silica flocculation rate constant.

\subsubsection{Tensile properties}
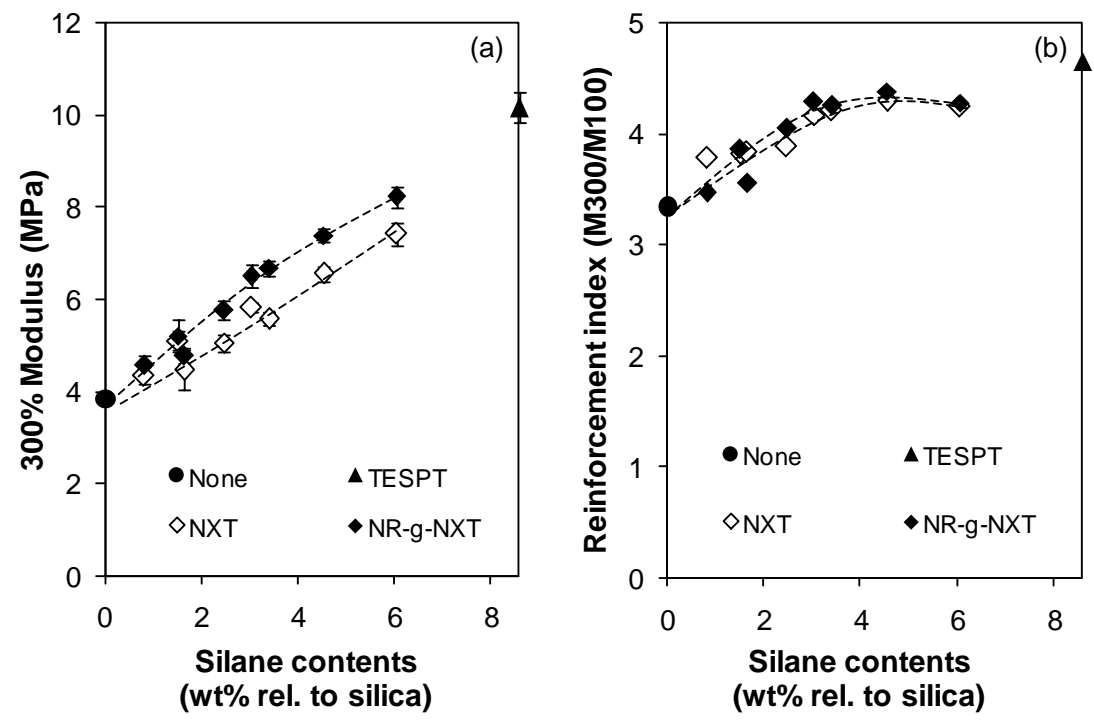

Figure 8.3 300\% Modulus (a) and reinforcement index (b) of silica-filled NR with straight use of NXT (open symbols) and NXT-grafted-NR (closed symbols).

The modulus at $300 \%$ elongation and reinforcement index of the silica-filled NR vulcanizates compatibilized by straight use of NXT in comparison with the use of NXTgrafted-NR are shown in Figures 8.3(a) and (b). The modulus of the vulcanized silica-filled NRs significantly increases with rising NXT-contents in both cases due to increase of the silica-rubber interactions and the use of NXT-grafted-NR as compatibilizer gives a higher modulus than the straight use of NXT, in accordance with the higher chemically bound rubber content: Figure 8.1(b), and lower Payne effect: Figure 8.2(a). The reinforcement index of the silica-filled NR vulcanizates also increases as a function of NXT-content: Figure 8.3(b), to reach an optimum at about $4.5 \mathrm{wt} \%$ of NXT relative to the silica, above which the addition of either more NXT or NXT-grafted-NR results in more or less the same values. In both cases the property levels of the TESPT-filled compound are not, respectively nearly reached. 

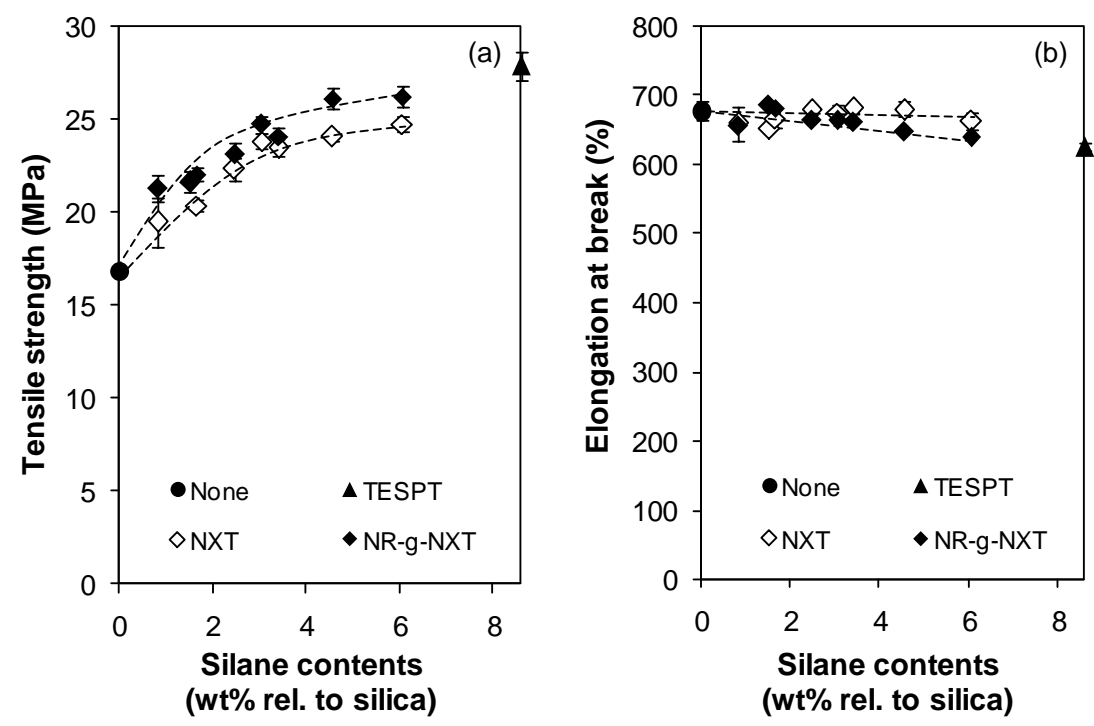

Figure 8.4 Tensile strength (a) and elongation at break (b) of silica-filled NR compounds with straight use of NXT (open symbols) and NXT-grafted-NR (closed symbols).

The tensile strength and elongation at break of the silica-filled NR vulcanizates with NXT-grafted-NR and straight NXT as compatibilizers show only small differences, where the tensile strength reaches a level slightly lower than that of the vulcanizate with $8.6 \mathrm{wt} \%$ of TESPT relative to the silica. The use of either NXT or NXT-grafted-NR clearly improves the tensile strength of the silica-filled NR, as a result of the increased silicarubber interactions by the coupling reaction of the NXT-silane. The tensile strength rises progressively when the NXT-content is increased up to about $3.5 \mathrm{wt} \%$ relative to the silica, and thereafter tends to level off. Like the 300\% modulus in Figure 8.3(a), the use of NXTgrafted-NR as compatibilizer gives a higher tensile strength than the straight use of NXTsilane. The coupling between silica and rubber that leads to the increased modulus and tensile strength affects the elongation at break slightly, decreasing a little as shown in Figure 8.4(b). 


\subsubsection{Surface topography}
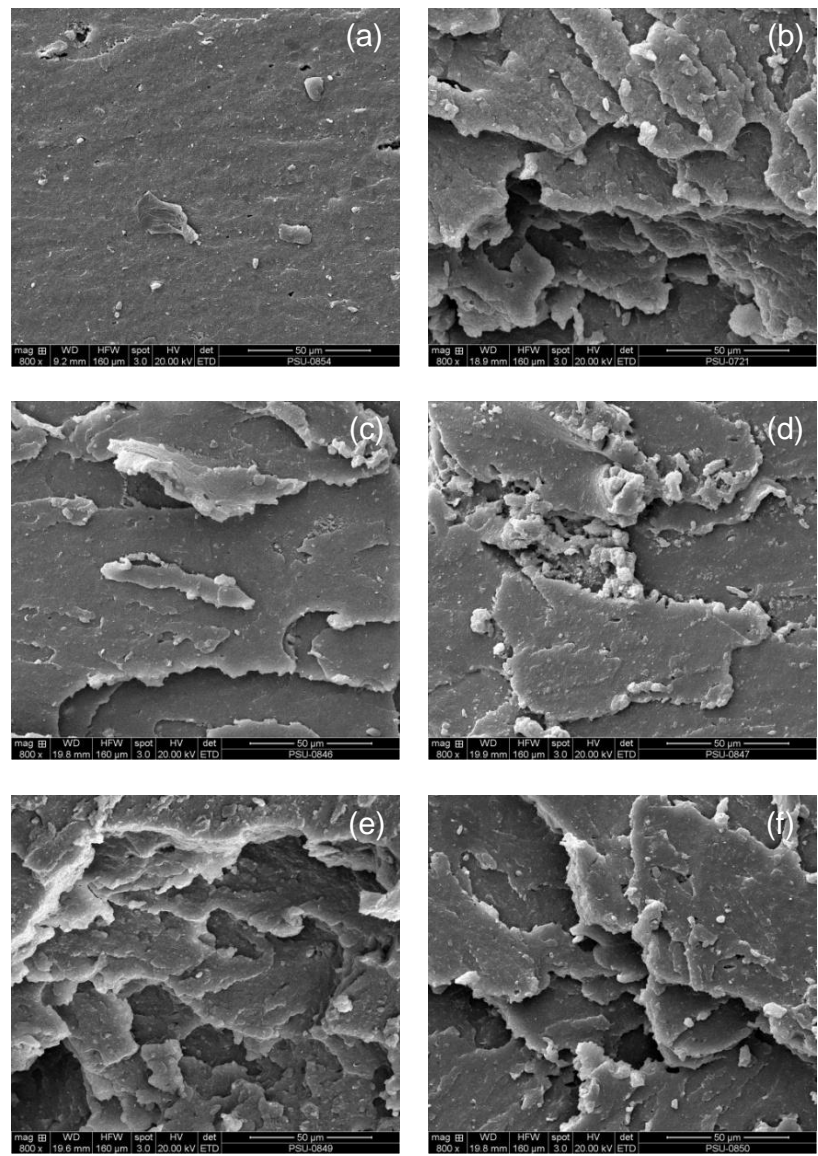

Figure 8.5 SEM micrographs of tensile fractured surfaces of silica-filled NR vulcanizates at 800x magnification: (a) without compatibilizer; (b) with TESPT at $8.6 \mathrm{wt} \%$ rel. to silica; (c) with NXT at 3.4 wt\% rel. to silica; (d) with NXT-grafted-NR containing total NXT of 3.4 wt\% rel. to silica; (e) with NXT at 6.1 wt\% rel. to silica; and (f) with NXT-grafted-NR containing total NXT of $6.1 \mathrm{wt} \%$ rel. to silica.

SEM micrographs of tensile fractured surfaces of silica-filled NR vulcanizates without compatibilizer, with TESPT, with NXT and NXT-grafted-NR are compared in Figures 8.5(a)-(f). As described in Chapters 3 and 4, a smooth failure surface of a silicafilled vulcanizate is due to a weak filler-rubber interaction and poor silica dispersion, whereas a rough failure surface with many tear lines from ductile failure indicates a good silica-rubber interaction and high tensile strength. The SEM micrographs of the 
vulcanizates with no compatibilizer and with reference TESPT, as shown in Figure 8.5(a) and (b) respectively, clearly correspond to their different tensile strengths: Figure 8.4(a). The addition of NXT improves silica-rubber interactions to lead to more surface roughness and tear lines on the failure surfaces when compared to the filled vulcanizate without compatibilizer. The increasing amount of NXT silane from 3.4 to $6.1 \mathrm{wt} \%$ relative to the silica results in ever more tear lines on the failure surfaces of the filled vulcanizates, as shown in Figure 8.5(c)-(f), due to more filler-rubber interaction and the higher strength, and the surface topography becomes more to resemble that of the reference compound with TESPT. However, there is no clear difference in the failure surface patterns of the filled vulcanizates prepared by the straight use of NXT and by the use of NXT-grafted-NR.

8.3.2 Effect of VP Si-363-grafted-NR as compatibilizer and straight use of VP Si363 on the properties of silica-filled NR compounds

8.3.2.1 Mooney viscosity, bound rubber contents, Payne effect and flocculation rate constant
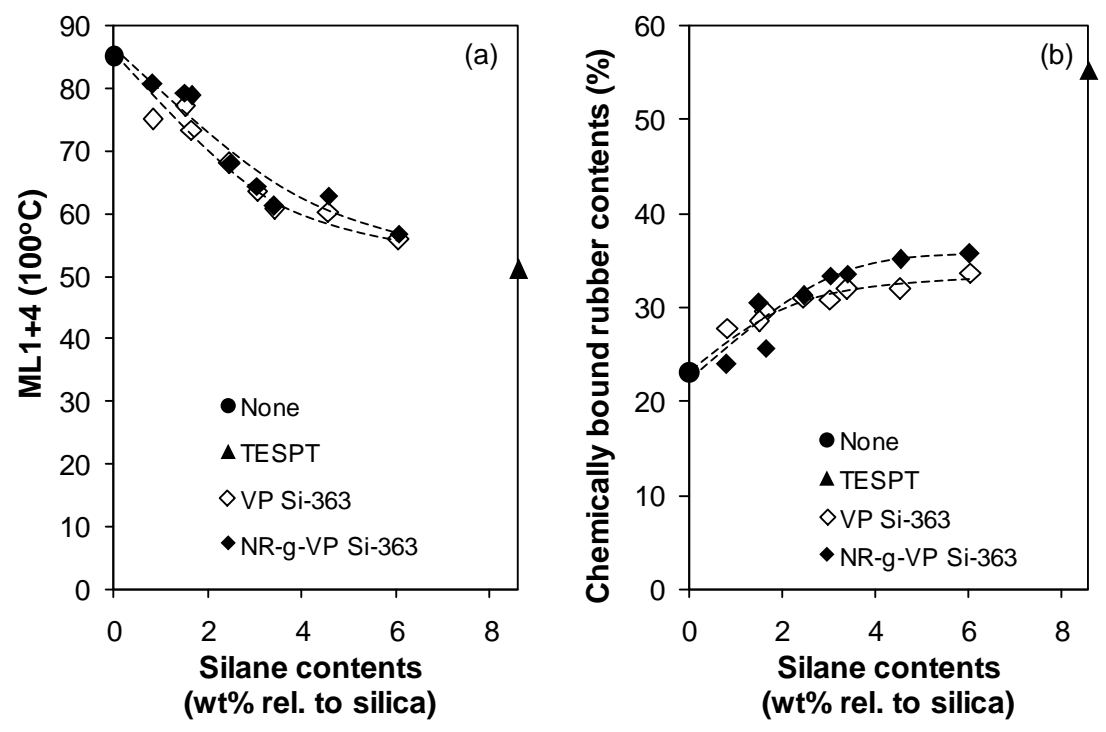

Figure 8.6 Mooney viscosity (a) and chemically bound rubber contents (b) of silica-filled NR with straight use of VP Si-363 (open symbols) and VP Si-363-grafted-NR (closed symbols). 
The Mooney viscosities of the silica-filled NR compounds are different depending on the types and amounts of compatibilizers; as shown in Figure 8.6(a). The incorporation of compatibilizers either in the form of straight VP Si-363 silane or VP Si-363-grafted-NR dramatically decreases the Mooney viscosity because of the improvement in silica-rubber interactions after the silanization reaction that lead to the improved silica dispersion, but there is no difference in Mooney viscosities between the two sets of compounds.

Figure 8.6(b) shows the chemically bound rubber contents after treatment in ammonia atmosphere to cleave all weak physical filler-rubber interactions. ${ }^{[29]}$ The results are similar to what has been observed in Figure 8.1(b) for NXT-silane: the increasing amount of VP Si-363 increases the chemically bound rubber content in the compounds to just a slightly lower level-off value than for NXT. As only some of the silanol groups will react with the ethoxy groups of silane, the adsorbed silane on the silica surface will also act as a shielding agent that only forms physical interactions between filler and rubber. The chemically bound rubber content therefore reaches a saturated point, as observed in Figures $8.1(\mathrm{~b})$ and $8.6(\mathrm{~b})$. The use of VP Si-363-grafted-NR shows a slightly higher chemically bound rubber content than the straight use of VP Si-363 in the range of high silane contents, i.e. $>3.5 \mathrm{wt} \%$ relative to the silica, due to some thiol-groups of the VP Si363 molecules already bonded to NR molecules prior to use, causing the higher extent of silica-rubber interactions.
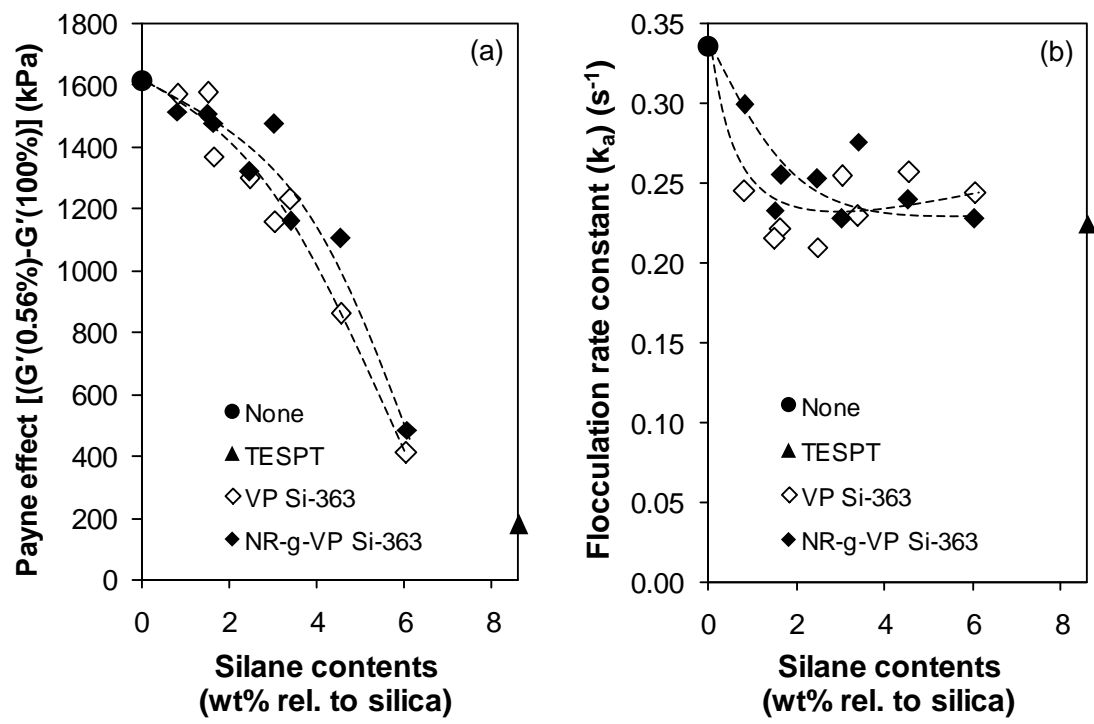

Figure 8.7 Payne effect (a) and flocculation rate constant (b) of silica-filled NR with straight use of VP Si-363 (open symbols) and VP Si-363-grafted-NR (closed symbols). 
The filler-filler interaction or Payne effect of the silica-filled NR compounds with various amounts of VP Si-363 silane is shown in Figure 8.7(a). Increasing VP Si-363 contents progressively reduce the Payne effect, and the use of silane either in its original form or in the silane-grafted-NR form results in similar properties. The flocculation rate constant $\left(k_{a}\right)$ or re-agglomeration rate of the silica under heat treatment is reduced after applying the compatibilizers, as shown in Figure 8.7(b), but the scatter in the data due to the measurement error is too large to significantly discriminate between the two different forms of silane. During the silanization reaction, while mixing the ethoxy groups of the silane are condensed with the silanol groups of silica to form a covalent bond between silica and silane if straight silane is used, or to possibly form a link between silica and rubber if silane-grafted-rubber is used. Moreover, the bulky alkoxy-groups with oxygen atoms of VP Si-363 can adsorb on the silica surface giving a shielding effect on the polar surface. Therefore, the filler-filler interactions as well as flocculation rate constant are decreased.

\subsubsection{Tensile properties}
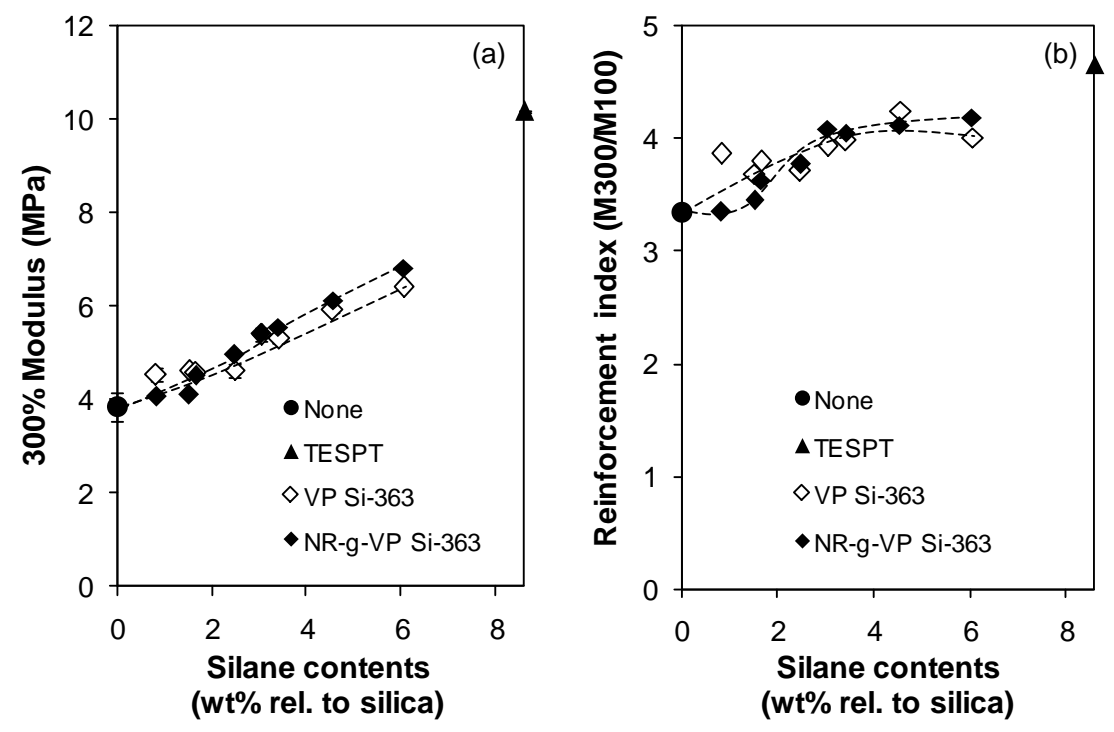

Figure $8.8300 \%$ Modulus (a) and reinforcement index (b) of silica-filled NR with straight use of VP Si-363 (open symbols) and VP Si-363-grafted-NR (closed symbols).

The 300\% modulus and reinforcement index (M300/M100) of the silica-filled NR vulcanizates containing different amounts of VP Si-363 in the form of straight silane and 
silane grafted NR, are shown in Figures 8.8(a) and (b) in comparison with those containing TESPT and no compatibilizer. The two sets of compounds that contain VP Si363 and VP Si-363-grafted-NR show no significant difference in $300 \%$ modulus and reinforcement index. The values increase with increasing VP Si-363 contents but to a lower level compared to the use of TESPT. The increasing filler-rubber interactions as derived form the chemically bound rubber contents: Figure 8.6(b), lead to a higher resistance to deformation, but because there are no additional crosslinks generated by sulfur donated to the compounds by the silane molecules like in the case of TESPT, the VP Si-363 sillanized compounds therefore have a significantly lower modulus.
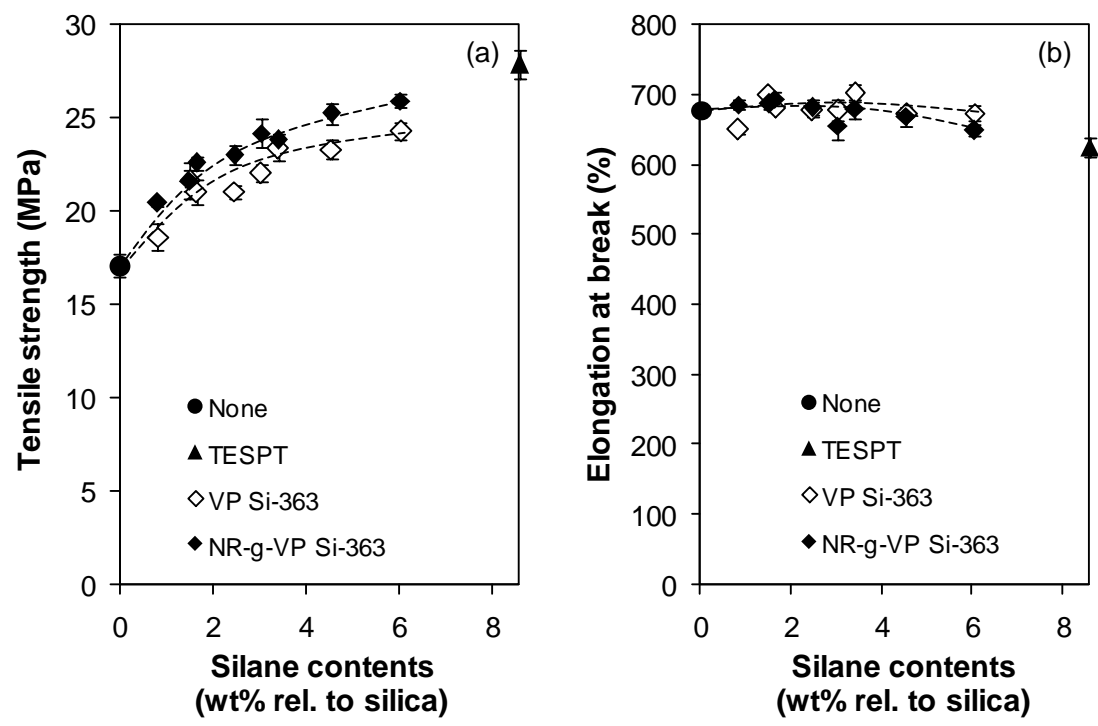

Figure 8.9 Tensile strength (a) and elongation at break (b) of silica-filled NR with straight use of VP Si-363 (open symbols) and VP Si-363-grafted-NR (closed symbols).

The tensile strength of the silica-filled vulcanizates also increases as a function of VP Si-363 content due to the effect-of the coupling reaction, where the use of VP Si-363grafted-NR as compatibilizer shows a better improvement in tensile strength especially at high VP Si-363-grafted-NR contents compared to the use of straight silane, as seen in Figure 8.9(a). The elongation at break of the silica-filled NR vulcanizates marginally decreases with increasing amount of VP Si-363, as shown in Figure 8.9(b), due to deformation restriction by silica-to-rubber linkages but the vulcanizates show slightly higher elongation at break than the reference TESPT vulcanizate that has more crosslinks by the sulfur released from its tetrasulfide structure. 


\subsubsection{Surface topography}
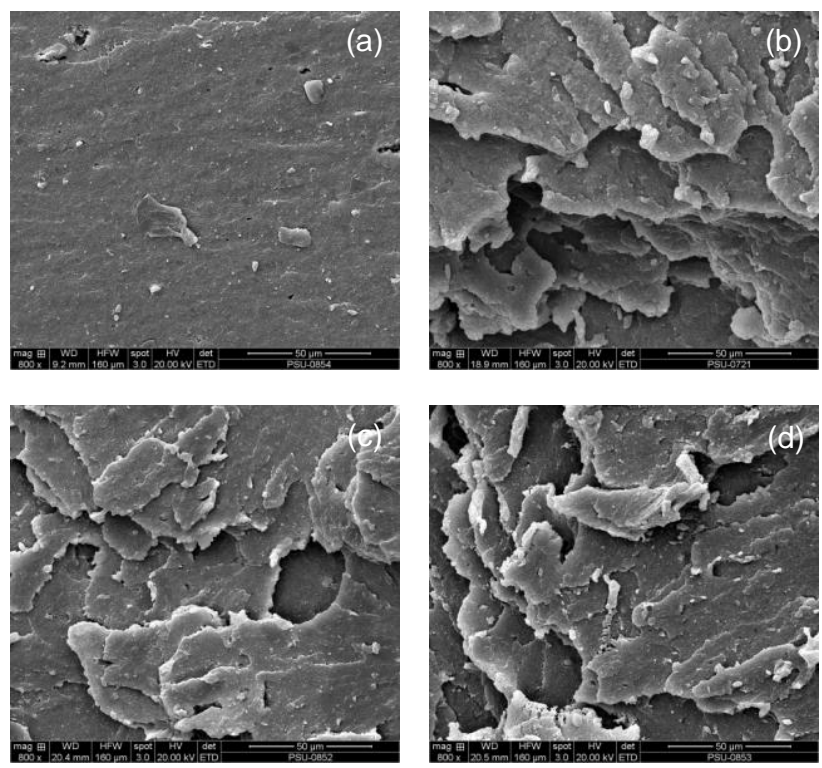

Figure 8.10 SEM micrographs of tensile fractured surfaces of silica-filled NR vulcanizates at $800 x$ magnification: (a) without compatibilizer; (b) with TESPT at $8.6 \mathrm{wt} \%$ rel. to silica; (c) with VP Si-363 at 6.1 wt\% rel. to silica; (d) with VP Si-363-grafted-NR containing total silane of $6.1 \mathrm{wt} \%$ rel. to silica.

SEM images of the tensile fractured surface of silica-filled NR vulcanizates with the use of straight VP Si-363 and VP Si-363 that was partially bonded to NR are shown in Figures 8.10 (c) and (d) in comparison with that of reference vulcanizates with no compatibilizer and with TESPT in Figures 8.12(a) and (b). The vulcanizates with TESPT and VP Si-363-grafted-NR show similar failure surfaces with many tear lines in accordance with their similar tensile strength, whereas the one with the use of VP Si-363 shows a slightly less complicated fracture surface indicating a somewhat less ductile behavior. The weakest silica-filled NR vulcanizate without compatibilizer in Figure 8.10(a) shows a smooth fracture surface due to its poor filler-rubber interactions that lead to easy sample rupture. 


\subsubsection{Comparative properties of silica-filled NR containing NXT-grafted-NR and} VP Si-363-grafted-NR as compatibilizers

The results in Sections 8.3.1 and 8.3.2 have shown that the use of silane-grafted NRs, i.e. NXT-grafted-NR and VP Si-363-grafted-NR, as compatibilizers give somewhat better overall properties of the silica-filled NR compounds compared to the straight use of free silane. In the silane-grafted NRs, part of the silanes is already grafted on the NRmolecules as proved in Chapter 7, and another part has remained free in the material. The improvement in the filled-rubber properties comes from a good filler-rubber interaction via the silanization reaction between the ethoxy-groups of the silanes and the silanol-groups of silica during mixing, where the pre-bonded silane and rubber via the grafting reaction may lead to a better coupling reaction during vulcanization that involves several chemicals and a complex reaction path. This part provides a comparison of the influence of NXTand VP Si-363-grafted-NRs on the properties of silica-filled NR compounds. Mooney viscosity, chemically bound rubber content, Payne effect, flocculation rate constant, $300 \%$ modulus, reinforcement index, tensile strength, elongation at break and dynamic mechanical properties are presented in comparison with those of the reference compounds with TESPT and without compatibilizer.
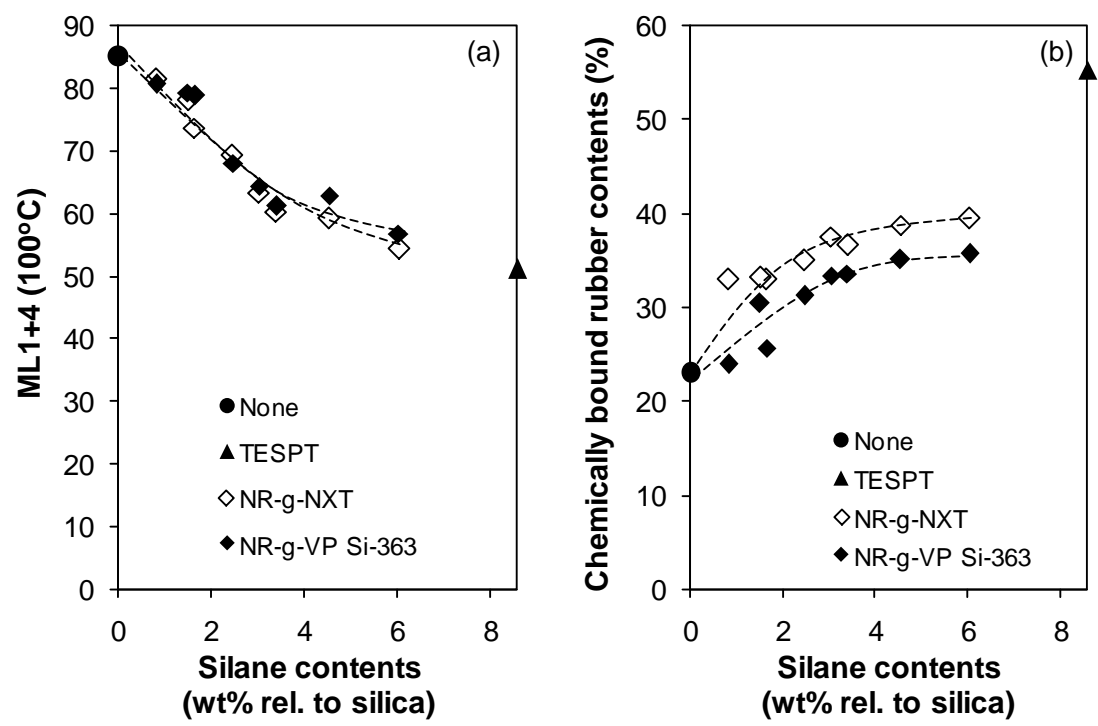

Figure 8.11 Mooney viscosity (a) and chemically bound rubber content (b) of silica-filled NR with NXT-grafted-NR (open symbols) and VP Si-363-grafted-NR (closed symbols). 
Mooney viscosities of the silica-filled NR compounds with NXT- and VP Si-363grafted-NRs decrease with increasing amounts of the compatibilizers, and both types of the silane-grafted NRs give very similar viscosity levels as shown in Figure 8.11(a). Figure 8.11(b) shows the chemically bound rubber contents of the silica-filled NR compounds in which the compounds with NXT-grafted-NR show a higher chemically bound rubber content than those with VP Si-363-grafted-NR.
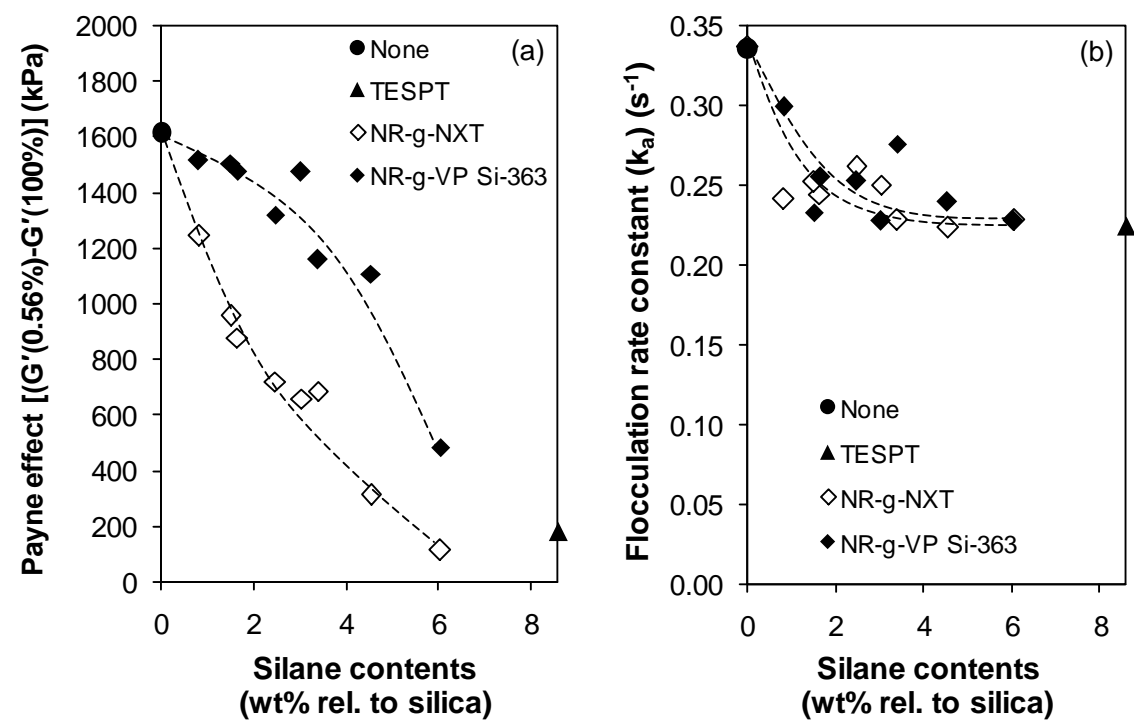

Figure 8.12 Payne effect (a) and flocculation rate constant (b) of silica-filled NR with NXTgrafted-NR (open symbols) and VP Si-363-grafted-NR (closed symbols).

The filler-filler interaction or Payne effect of the silica-filled NR compounds remarkably decreases with increasing silane contents in the NXT- and VP Si-363-graftedNRs, as shown in Figure 8.12(a). The use of NXT-grafted-NR results in a clearly lower Payne effect of the filled compounds compared to the use of VP Si-363-grafted-NR. At 6.1 $w t \%$ of NXT, the compound shows a slightly lower Payne effect compared to the reference compound with 8.6 wt\% of TESPT. Different silanes have different efficiencies for silicareinforced compounds. At equal weight, NXT that has a lower molecular weight and three ethoxy-groups per molecule, shall have more functional groups available on a molar basis to react with the silanol groups of silica. As reported in Chapter 6, even at the same amount of functional groups, the use of NXT silane still provides a better improvement in the Payne effect than VP Si-363 due to its smaller molecule and less steric hindrance for the silanization reaction. Figure 8.12 (b) shows the flocculation rate constants of the silica- 
filled compounds with different types of compatibilizer. The addition of both types of compatibilizer at only a small amount reduces the flocculation rate of the compounds to a similar level as that of the TESPT-compound. However, the scatter in the data is too large to discriminate between the NR-g-NXT and NR-g-VP Si-363.
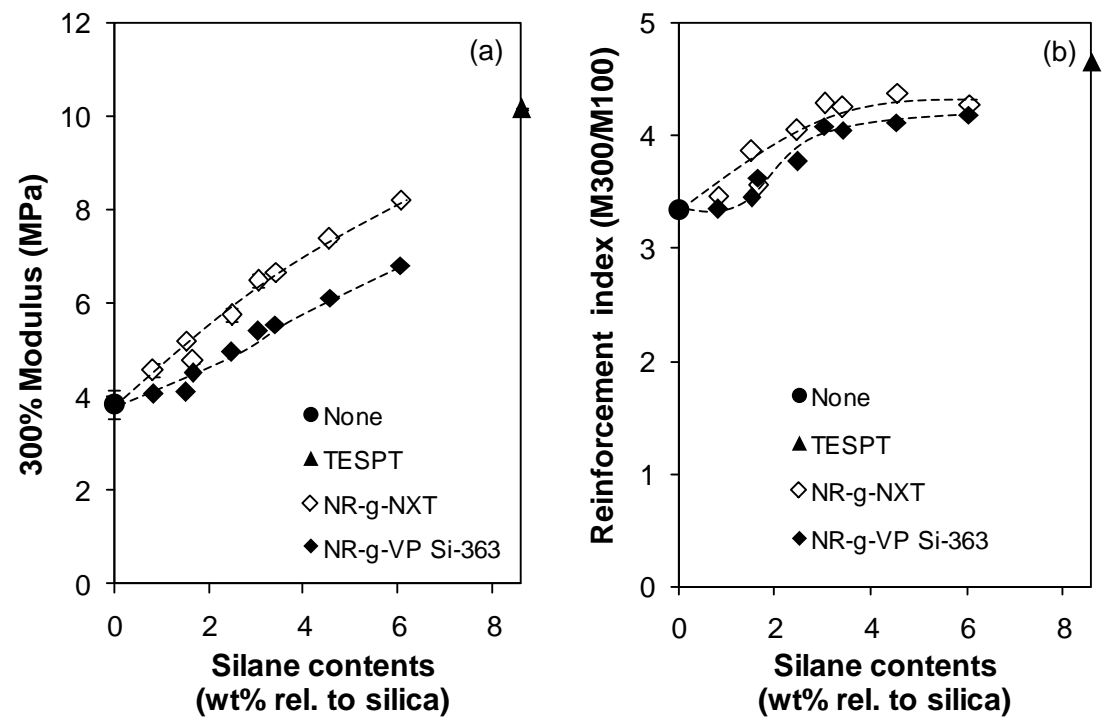

Figure 8.13 300\% Modulus (a) and reinforcement index (b) of silica-filled NR with NXTgrafted-NR (open symbols) and VP Si-363-grafted-NR (closed symbols).

The $300 \%$ modulus and reinforcement index of the silica-filled NR vulcanizates increase with the addition of increasing amounts of silane in the grafted NRs as displayed in Figures 8.13(a) and (b), respectively. This corresponds with the increased filler-rubber interaction and decreased Payne effect. The incorporation of NXT-grafted-NR leads to a clearly higher $300 \%$ modulus than the use of VP Si-363-grafted-NR, indicating a higher reinforcement efficiency of NXT for silica-reinforced NR. The NXT silane consists of more reactive ethoxy-groups to form a bond with the silanol-groups on the silica surface compared to the VP Si-363 silane. Moreover, at equal total silane content in the compounds, the higher grafting efficiency of the grafted-NXT on NR should result in a higher level of crosslinks or chemical bonds between rubber and filler, leading to a higher $300 \%$ modulus and reinforcement index. 

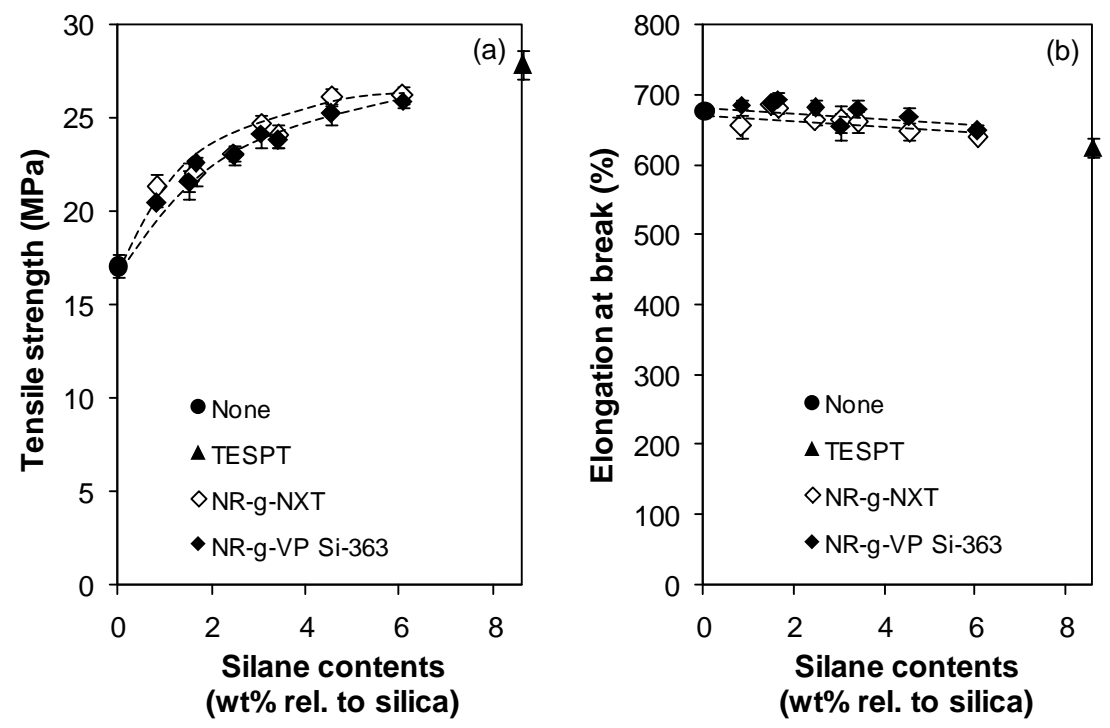

Figure 8.14 Tensile strength (a) and elongation at break (b) of silica-filled NR with NXTgrafted-NR (open symbols) and VP Si-363-grafted-NR (closed symbols).

The tensile strength of the silica-filled NR vulcanizates is enhanced with increasing total silane content in the grafted-NRs in correspondence with the increased modulus and reinforcement index as shown in Figure 8.14(a). However, among the silane types studied, TESPT still provides the best silica reinforcement for the NR compounds. The use of NR-g-NXT and NR-g-VP Si-363 as compatibilizers results in more or less the same elongation at break and increasing the total silane content decreases the extensibility of the vulcanizate to the level of the reference TESPT system as shown in Figure 8.14(b), due to the increase of linkages within the materials.

For the vulcanizates containing a total silane content of $6.1 \mathrm{wt} \%$ relative to the silica, with NR grafted by using 20 phr of silane, the dynamic mechanical properties were analyzed and the results are shown in Figures 8.15 and 8.16 in comparison with the ones with TESPT at $8.6 \mathrm{wt} \%$ relative to the silica and without compatibilizer. 

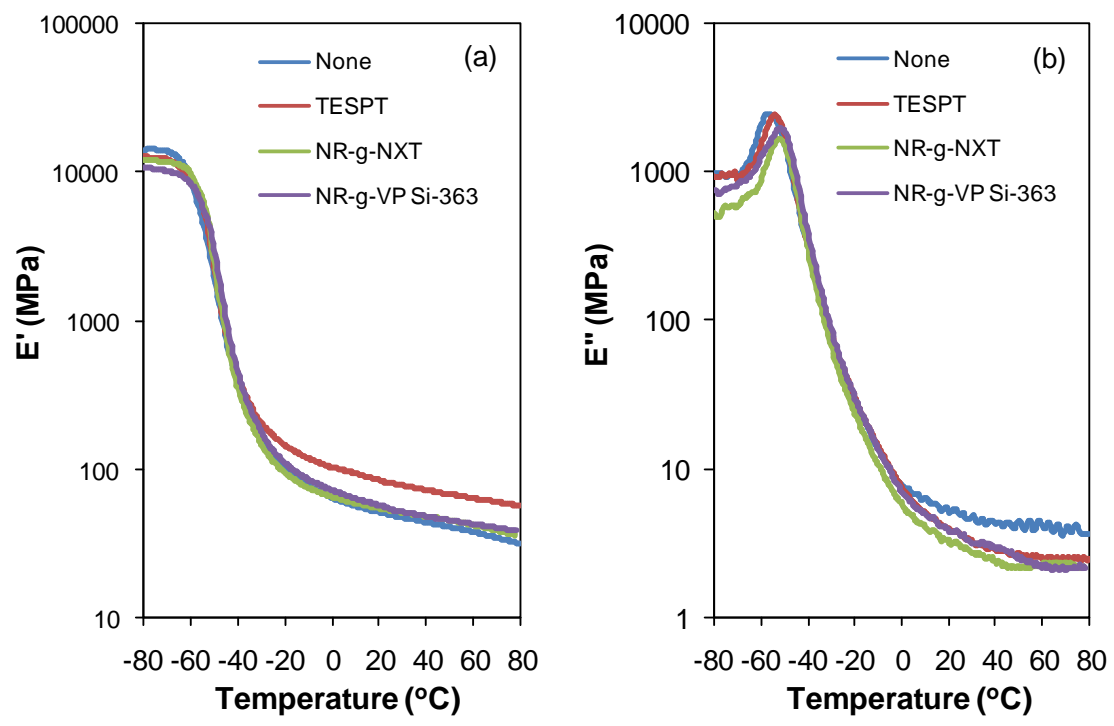

Figure 8.15 Storage modulus ( $\left.E^{\prime}\right)(a)$ and loss modulus (E") (b) of silica-filled NR with NXT- and VP Si-363-grafted-NRs, both with total silane of $6.1 \mathrm{wt} \%$ relative to the silica; TESPT at $8.6 \mathrm{wt} \%$ relative to silica.

The storage ( $\left.E^{\prime}\right)$ and loss (E") moduli as function of temperature of the silica-filled $\mathrm{NR}$ vulcanizates are shown in Figures 8.15(a) and (b), respectively. In the glassy region, the silica-filled vulcanizate with TESPT shows only small difference in storage modulus compared to the mixes with NXT- and VP Si-363-grafted NRs. But in the rubbery region, the silica/TESPT system shows the highest storage modulus while the other vulcanizates show similar levels of the moduli. For filled rubber, the modulus of the materials depends on filler content and filler-filler interactions that affect the segmental mobility of the rubber chains, and the modulus normally increases with increasing amount of filler ${ }^{[30]}$ or degree of filler-rubber interaction. ${ }^{[31]}$ The silica-filled NR with TESPT that has the highest filler-rubber interactions therefore shows the highest storage modulus. On the contrary, the silica-filled $\mathrm{NR}$ without compatibilizer that has the highest filler-filler interaction or Payne effect; Figure 8.12, and a filler-filler network with trapped and less flexibility of the chains to relocate under deformation ${ }^{[31]}$ shows the highest loss modulus in the rubbery region, i.e. the highest loss of energy as dissipated in heat. The incorporation of TESPT, NXT- or VP Si363-grafted-NRs results in less filler-filler interaction; Figure 8.12(a), and higher chemically bound rubber content; Figure $8.11(\mathrm{a})$, leads to a better elasticity and lower loss modulus. 


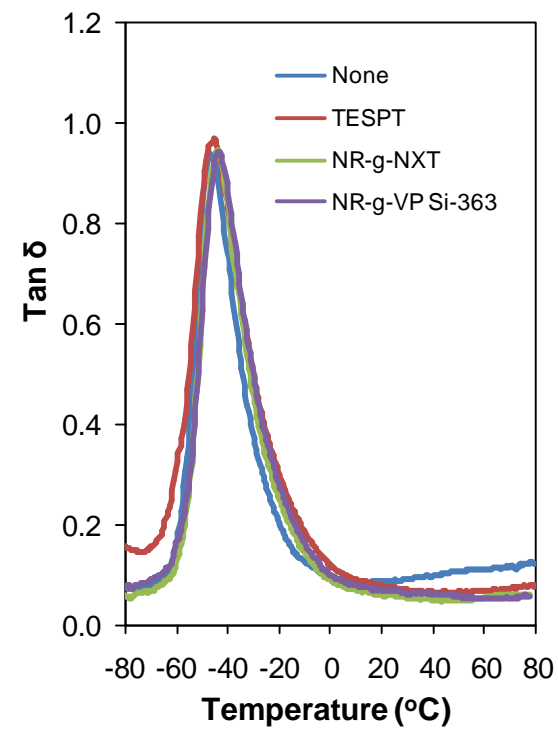

Figure 8.16 Tan $\delta$ of silica-filled NR with NXT- and VP Si-363-grafted-NRs, both with total silane of $6.1 \mathrm{wt} \%$ relative to the silica; TESPT at $8.6 \mathrm{wt} \%$ relative to silica.

The loss tangent or tan $\delta$ as a function of temperature of the silica-filled NR vulcanizates with different compatibilizers are shown in Figure 8.16, wherein the rubber with TESPT shows a slightly higher tan $\delta$ peak intensity over the other vulcanizates, which indicates that there is less trapped rubber in the silica network and so more flexible rubber chains to respond to the dynamic deformation in the transition region. In the rubbery region where the rubber chains are in motion, the compatibilized systems with the three types of compatibilizers show lower tan $\delta$ values compared to the non-compatibilized one, due to their better filler-rubber interactions and so less energy loss during deformation. The values of tan $\delta$ at different positions are summarized in Table 8.5 together with the values of $T_{g}$ taken from the tan $\delta$ and $E "$ peaks.

Table $8.4 \mathrm{~T}_{\mathrm{g}}$ and $\tan \delta$ values of silica-filled NR vulcanizates at $6.1 \mathrm{wt} \%$ relative to the silica for NR-g-NXT and NR-g-VP Si-363; and 8.6 wt\% for TESPT.

\begin{tabular}{lccccc}
\hline \multirow{2}{*}{ Compatibilizer types } & \multicolumn{2}{c}{$\mathrm{T}_{\mathrm{g}}\left({ }^{\circ} \mathrm{C}\right)$} & \multicolumn{3}{c}{ Values of Tan $\delta$} \\
\cline { 2 - 6 } & at $\tan \delta$ peak & at E" peak & at peak & at $5^{\circ} \mathrm{C}$ & at $60^{\circ} \mathrm{C}$ \\
\hline Without compatibilizer & -47 & -58 & 0.94 & 0.09 & 0.11 \\
TESPT & -45 & -54 & 0.97 & 0.10 & 0.07 \\
NR-g-NXT & -44 & -52 & 0.95 & 0.08 & 0.05 \\
NR-g-VP Si-363 & -44 & -52 & 0.95 & 0.08 & 0.05 \\
\hline
\end{tabular}


The glass transition temperature $\left(\mathrm{T}_{\mathrm{g}}\right)$ is the temperature of the glass-to-rubber softening transition which can be observed as a large drop in modulus. Even though the $T_{g}$ is often taken at the peak of tan $\delta$, the $T_{g}$ of a polymer can be reported by using the temperature at either the loss modulus (E") peak or at the tan $\delta$ peak, in which the value at the $E$ " peak corresponds to segmental relaxation processes only and is not affected by different levels of filler-rubber interaction. ${ }^{[32]}$ This present work compares the $\mathrm{T}_{\mathrm{g}}$ 's of the silica-filled NRs with different compatibilizers taken from both the $E^{\prime \prime}$ and tan $\delta$ peaks, which show the same trend, as shown in Table 8.4. That is, the $T_{g}$ 's of the silica-filled vulcanizates with TESPT, NXT- and VP Si-363-grafted-NRs are shifted to a higher temperature compared to the one without compatibilizer, due to an increased degree of filler-rubber interactions which restrict the mobility of the polymer chains. Although the compounds with NXT- and VP Si-363-grafted-NRs as compatibilizers show lower chemically bound rubber content and inferior mechanical properties than for TESPT, their $\mathrm{T}_{\mathrm{g}}$ 's are slightly higher than the one with TESPT. The use of silane grafted NR with some silane molecules already bonded to rubber may result in a higher extent of the coupling reaction between rubber and silica. In the transition region, the free volume between polymer molecules rapidly increases leading to an increase of polymer chain mobility to respond to dynamic deformation. The better chain flexibility in the compatibilized compounds and good filler-rubber interactions lead to lower energy losses and so lower $\tan \delta$ in the rubbery region.

As mentioned in the previous chapters, the tan $\delta$ values at low temperature, i.e. $5-35^{\circ} \mathrm{C}$ for summer tires and $2-20^{\circ} \mathrm{C}$ for winter tires ${ }^{[3]}$, and at higher temperatures commonly at $60^{\circ} \mathrm{C}$ can be used to indicate tire wet grip and rolling resistance, respectively. The results in Table 8.4 shows that the use of NXT- and VP Si-363-graftedNRs as compatibilizers give slightly lower tan $\delta$ values at $5^{\circ} \mathrm{C}$, i.e. a small decrease in wet grip, but lower tan $\delta$ at $60^{\circ} \mathrm{C}$ implying an improvement in rolling resistance compared to the reference compound with TESPT. The lower tan $\delta$ at $60^{\circ} \mathrm{C}$ in the vulcanizates containing the silane grafted NR again may be attributed to efficient coupling or bridging between rubber and silica via the pre-grafted silane fragments and some additional linkages created by self-crosslinking between the grafted moieties.

Overall, the use of silane-grafted-NRs as compatibilizers in the silica-filled NR compounds without sulfur compensation can effectively enhance the properties compared to the non-compatibilized system. By taking the compound with $8.6 \mathrm{wt} \%$ TESPT relative to the silica as reference, the use of NXT-grafted-NR that gives better performance than VP Si-363 with 4-6 wt\% of silane relative to the silica, gives similar Mooney viscosity, Payne effect and flocculation rate constant, but inferior chemically bound rubber content, 
modulus and tensile strength. However, a slight shift in the $T_{g}$ of the vulcanizate in the presence of NXT-grafted-NR gives a positive effect on tire rolling resistance as indicated by the lower $\tan \delta$ at $60^{\circ} \mathrm{C}$.

\subsection{Conclusions}

Increasing amounts of NXT- and VP Si-363-grafted-NRs as compatibilizers in silica-filled NR-compounds decrease the Mooney viscosity, filler-filler interaction or Payne effect, flocculation rate constant and increase the chemically bound rubber contents, $300 \%$ modulus, reinforcement index and tensile strength. The reinforcement index and tensile strength of the silica-filled NR vulcanizates almost level off when the silane content in the silane-grafted-NRs, which includes free and grafted silane, exceeds 3.0 wt\% relative to the silica. Tensile fractured surfaces of the silica-filled NR vulcanizates indicate ductile failure with surface roughness and many tear lines when the silane-grafted-NRs are used, similar to the compound with TESPT. At the same silane loading, the use of silanegrafted-NR as compatibilizer gives a better improvement in Payne effect, chemically bound rubber content, $300 \%$ modulus and tensile strength compared to the straight use of a silane. Comparing the two types of silane-grafted-NRs, the use of NXT provides a better improvement of overall properties than VP Si-363, but the properties are still somewhat lower than for the use of TESPT at its optimum content, i.e. at $8.6 \mathrm{wt} \%$ relative to the silica. In the presence of the silane-grafted-NR as compatibilizer, the $T_{g}$ of the silica-filled NR vulcanizates is shifted to higher temperature. Tan $\delta$ at $5^{\circ} \mathrm{C}$ that relates to wet grip is slightly decreased but the tan $\delta$ at $60^{\circ} \mathrm{C}$ is clearly reduced indicating a lower tire rolling resistance for tire treads made thereof, compared to the reference compound with TESPT.

\subsection{References}

1. I.R. Gelling, Rubber Chem. Technol., 58, 86 (1985).

2. A.K. Manna, A.K. Bhattacharyya, P.P De, D.K. Tripathy, S.K. De, D.G. Peiffer, Polymer, 39, 7113 (1998).

3. A.K. Manna, P.P. De, D.K. Tripathy, S.K. De, D.G. Peiffer, J. Appl. Polym. Sci., 74, 389 (1999).

4. M.M. Jacobi, M.V. Braum, T.L.A.C. Rocha, R.H. Schuster, Kautsch. Gummi Kunstst., 60, 460 (2007).

5. T.L.A.C. Rocha, R.H. Schuster, M.M. Jacobi, D. Samios, Kautsch. Gummi Kunstst., 57, 656 (2004).

6. A.K. Manna, P.P. De, D.K. Tripathy, J. Appl. Polym. Sci., 84, 2171 (2002). 
7. Y.-T. Shieh, T.-H. Tsai, J. Appl. Polym. Sci., 69, 255 (1998).

8. K. Sirisinha, M. Boonkongkaew, S. Kositchaiyong, Polym. Test., 29, 958 (2010).

9. M. Beltrán, C. Mijangos, Polym. Eng. Sci., 40, 1534 (2000).

10. Y.-T. Shieh, C.-M. Liu, J. Appl. Polym. Sci., 74, 3404 (1999).

11. S. Jain, J.G.P. Goossens, M. van Duin, Macromol. Symp., 233, 225 (2006).

12. C. Yin, Q. Zhang, J. Gu, J. Zheng, G. Gong, T. Liang, H. Zhang, J. Appl. Polym. Sci., 128, 2262 (2013).

13. C. Yin, Q. Zhang, D. Yin, J. Gu, G. Gu, T. Liang, J. Appl. Polym. Sci., 119, 2808 (2011).

14. G. Colucci, A.D. Gianni, R. Bongiovanni, A. Priola, F. Torello, S. Bracco, P. Sozzani, Macromol. Symp., 296, 550 (2010).

15. A. Bertora, M. Castellano, E. Marsano, M. Alessi, L. Conzatti, P. Stagnaro, G. Colucci, A. Priola, A. Turturro, Macromol. Mater. Eng., 296, 455 (2011).

16. F. Schapman, J.P. Couvercelle, C. Bunel, Polymer, 39, 4955 (1998).

17. S.-S. Choi, Korea Polym. J., 8, 73 (2000).

18. A. Ansarifar, S.F. Shiah, M. Bennett, Int. J. Adhes. Adhes., 26, 454 (2006).

19. S. Schaal, A.Y. Coran, Rubber Chem. Technol., 73, 225 (2000).

20. S. Choi, B.-H. Park, H. Song, Polym. Adv. Technol., 15, 122 (2004).

21. S. Wolff, M.-J. Wang, Rubber Chem. Technol., 65, 329 (1992).

22. S.-S. Choi, C. Nah, B.-W. Jo, Polym. Int., 52, 1382 (2003).

23. S.-S. Choi, J. Appl. Polym. Sci., 79, 1127 (2001).

24. C.Jr. Lin, W.L. Hergenrother, A.S. Hilton, Rubber Chem. Technol., 75, 215 (2002).

25. L. Guy, S. Daudey, P. Cochet, Y. Bomal, Kautsch. Gummi Kunstst., 62, 383 (2009).

26. W. Kaewsakul, K. Sahakaro, W.K. Dierkes, J.W.M. Noordermeer, Rubber Chem. Technol., 86, 313 (2013).

27. S. Mihara, R.N. Datta, J.W.M. Noordermeer, Rubber Chem. Technol., 82, 524 (2009).

28. H.-D. Luginsland, J. Fröhlich, A. Wehmeier, Rubber Chem. Technol., 75, 563 (2002).

29. S. Wolff, M.-J. Wang, E.-H. Tan, Rubber Chem. Technol., 66, 163 (1993).

30. C. Gauthier, E. Reynaud, R. Vassoille, L. Ladouce-Stelandre, Polymer, 45, 2761 (2004).

31. H.M. Da Costa, L.L.Y. Visconte, R.C.R. Nunes, C.R.G. Furtado, J. Appl. Polym. Sci., 83, 2331 (2002).

32. C.G.Robertson, C.J. Lin, M. Rackaitis, C.M. Roland, Macromolecules, 41, 2727 (2008).

33. UNECE Regulation No 117, GRRF 71 ${ }^{\text {st }}$ Session, September 13-15, 2011. 


\section{Compatibilization of Silica-Filled Natural Rubber by Using Silane-Grafted-Rubber with Compensation of Sulfur}

Sulfur compensation is applied to the silica-filled NR compounds with the NXTgrafted-NR as compatibilizer and the properties are investigated in comparison with those of the silica-filled NR with TESPT and without compatibilizer. The non-purified NXTgrafted-NRs were used as part of the rubber matrix in a range of 5-20 phr, so as to give total NXT contents of 0.8-6.1 wt\% relative to the silica. The compounds with sulfur compensation show slightly lower Mooney viscosity, similar Payne effect and flocculation rate constant but higher cure torque difference compared to the compounds without extra sulfur. After vulcanization, the rubber with NR-g-NXT at $6 w t \% N X T$ relative to the silica and with sulfur compensation shows almost the same levels of $300 \%$ modulus, reinforcement index, tensile strength and elongation at break, compared to the reference compound with 8.6 wt\% TESPT relative to the silica. Tensile fractured surfaces of the vulcanizates with sulfur compensation show more tear lines, which correlates with their tensile strength. The use of NR-g-NXT as compatibilizer increases $T_{g}$ of the vulcanizates slightly and sulfur compensation causes a slight further shift of $T_{g}$ to a higher temperature. 


\subsection{Introduction}

The results in Chapter 8 have shown that the use of NXT-grafted-NR as compatibilizer gives better properties than the straight use of NXT and the use of VP Si363-grafted-NR. However, due to the difference in sulfur amounts in the compounds, the properties of the NXT compatibilized compounds are still inferior to those of the reference TESPT compound. In Chapter 4, it has been demonstrated for the silica-filled NR compounds compatibilized with ENR and a small amount of TESPT that sulfur compensation relative to the reference compound with TESPT alone could further improve the properties such as crosslink density, modulus and tensile strength. The use of epoxidized low molecular weight NR as compatibilizer for silica-filled NR also showed that, even though the addition of compatibilizer could improve both compound and vulcanizate properties, the addition of extra sulfur to compensate for the sulfur released from silane molecules remarkably enhanced the $300 \%$ modulus, tensile strength and loss tangent at $60^{\circ} \mathrm{C}$ due to the better network formation. ${ }^{[1]}$

A number of previous studies reported the same with regard to the influence of sulfur content in the silica-reinforced rubber compounds. Ten Brinke et al. ${ }^{[2]}$ showed that, with sulfur correction, all sulfur containing silanes behaved more like TESPT; such as bis(triethoxysilylpropyl)disulfide (TESPD) with sulfur correction could give final properties similar to those of TESPT. A mechanistic study of the role of coupling agents in silicarubber compounds ${ }^{[3]}$ reported that the carbon and sulfur chain lengths within the coupling agents prominently influence the tensile properties. Sulfur in the silanes plays a dual role: one is as part of the coupling agent to attach to the rubber, another role is as direct curative. The addition of extra sulfur to the TESPT-silica-NR significantly changes the overall properties of both compounds and vulcanizates. The correction of sulfur deficiency compared to the TESPT-based system leads to enhanced properties which are related to extra network formation. ${ }^{[4]}$

This Chapter reports the effect of sulfur compensation relative to the sulfur contained in the reference TESPT compound in order to further improve the properties of the silica-filled NR compounds with NXT-grafted-NR as compatibilizer.

\subsection{Experimental}

\subsubsection{Materials and compound preparation}

All of the materials used for compound preparation in this chapter, i.e. natural rubber, silica, TESPT, TDAE oil, TMQ, zinc oxide, stearic acid, CBS, DPG and sulfur, 
were the same as described in Chapter 3. The NXT-grafted-NR was prepared and characterized as detailed in Chapter 7.

The silica-filled NR compounds were prepared using the formulations as shown in Table 9.1, and the mixing procedures as described in Table 9.2. The pre-prepared NXTgrafted-NRs were used without purification at the varied amounts at 5, 10, 15 and $20 \mathrm{phr}$ to give the different total silane contents as shown in Table 8.1 in Chapter 8. The compounds were mixed in an internal mixer with the initial mixer temperature setting of $100^{\circ} \mathrm{C}$ to complete the silanization of silica and silane, as explained in Chapter 5 . The reference compounds with $8.6 \mathrm{wt} \%$ TESPT relative to the silica and without compatibilizer were also prepared according to the formulations as shown in Table 8.2 and the mixing procedures in Table 8.3.

Table 9.1 Compound formulations with the use of NR-g-NXT and sulfur compensation.

\begin{tabular}{|c|c|c|c|c|c|c|c|c|}
\hline \multirow{3}{*}{$\begin{array}{l}\text { Ingredients } \\
\text { RSS3 }\end{array}$} & \multicolumn{8}{|c|}{ Parts per hundred parts of rubber (phr) } \\
\hline & \multicolumn{4}{|c|}{$\begin{array}{c}\text { NXT-grafted-NR with silane } \\
10 \mathrm{phr}\end{array}$} & \multicolumn{4}{|c|}{$\begin{array}{c}\text { NXT-grafted-NR with silane } \\
20 \mathrm{phr}\end{array}$} \\
\hline & 95.0 & 90.0 & 85.0 & 80.0 & 95.0 & 90.0 & 85.0 & 80.0 \\
\hline TESPT & - & - & - & - & - & - & - & - \\
\hline NXT-grafted-NR & 5.0 & 10.0 & 15.0 & 20.0 & 5.0 & 10.0 & 15.0 & 20.0 \\
\hline Zeosil 1165MP & 55.0 & 55.0 & 55.0 & 55.0 & 55.0 & 55.0 & 55.0 & 55.0 \\
\hline TDAE oil & 8.0 & 8.0 & 8.0 & 8.0 & 8.0 & 8.0 & 8.0 & 8.0 \\
\hline $\mathrm{ZnO}$ & 3.0 & 3.0 & 3.0 & 3.0 & 3.0 & 3.0 & 3.0 & 3.0 \\
\hline TMQ & 1.0 & 1.0 & 1.0 & 1.0 & 1.0 & 1.0 & 1.0 & 1.0 \\
\hline Stearic acid & 1.0 & 1.0 & 1.0 & 1.0 & 1.0 & 1.0 & 1.0 & 1.0 \\
\hline DPG & 1.0 & 1.0 & 1.0 & 1.0 & 1.0 & 1.0 & 1.0 & 1.0 \\
\hline CBS & 1.5 & 1.5 & 1.5 & 1.5 & 1.5 & 1.5 & 1.5 & 1.5 \\
\hline Sulfur ${ }^{*}$ & 2.59 & 2.55 & 2.51 & 2.47 & 2.56 & 2.48 & 2.41 & 2.33 \\
\hline \multicolumn{9}{|c|}{$\begin{array}{l}\text { Remarks: *Sulfur content was compensated for the amount of sulfur contained in TESPT in the } \\
\text { reference compound. }\end{array}$} \\
\hline \multicolumn{9}{|c|}{ Table 9.2 Mixing procedures. } \\
\hline \multicolumn{5}{|c|}{ Mixing procedures } & \multicolumn{4}{|c|}{ Cumulative time (mins.) } \\
\hline \multicolumn{9}{|c|}{ Step $1:$ Internal mixer } \\
\hline \multicolumn{6}{|c|}{ - NR and silane-grafted-NR mastication } & \multicolumn{3}{|c|}{2} \\
\hline \multicolumn{6}{|c|}{ - Addition of first half of silica } & \multicolumn{3}{|c|}{7} \\
\hline \multicolumn{6}{|c|}{ - Addition of second half of silica and TDAE oil } & \multicolumn{3}{|c|}{12} \\
\hline \multicolumn{6}{|c|}{ - Addition of ZnO, stearic acid, and TMQ } & \multicolumn{3}{|c|}{15} \\
\hline \multicolumn{9}{|c|}{ Step 2 : Two roll mill } \\
\hline \multicolumn{6}{|c|}{ - Addition of DPG, CBS, and sulfur (+ extra sulfur) } & \multicolumn{3}{|c|}{5} \\
\hline
\end{tabular}




\subsubsection{Property testing and characterization}

Mooney viscosity of the compounds after step 2 and cure characteristics were tested according to the methods as described in Chapter 5. Payne effect and flocculation rate constant of the final compounds (with curatives) were analyzed according to the methods as detailed in Chapter 3. Bound rubber content of the non-productive compounds after step 1 was analyzed following the procedures as described in Chapter 3.

The compounds were press-cured to their respective optimum cure times $\left(t_{c 90}\right)$ and tensile as well as dynamic mechanical properties were tested following the methods as described in Chapter 5. Tensile fractured surfaces were analyzed by using the scanning electron microscopy (SEM) technique, as described in Chapter 4.

\subsection{Results and discussion}

\subsubsection{Mooney viscosity, bound rubber content, Payne effect and flocculation} rate constant

The Mooney viscosity of the silica-filled compound is affected by the degree of silica dispersion and silica-rubber interactions in a rubber matrix which in turn may be correlated to other properties. The presence of a compatibilizer which is adsorbed onto the silica surface to increase its hydrophobic character results in a suppression of the silica-silica network and improves the silica dispersion in the rubber matrix, leading to a decrease of Mooney viscosity of the silica-filled compounds. ${ }^{[5]}$ On the other hand, an increase of the Mooney viscosity may also be observed if the filled-compound shows a significant improvement in filler-rubber interactions by impeded rubber segmental mobility. 

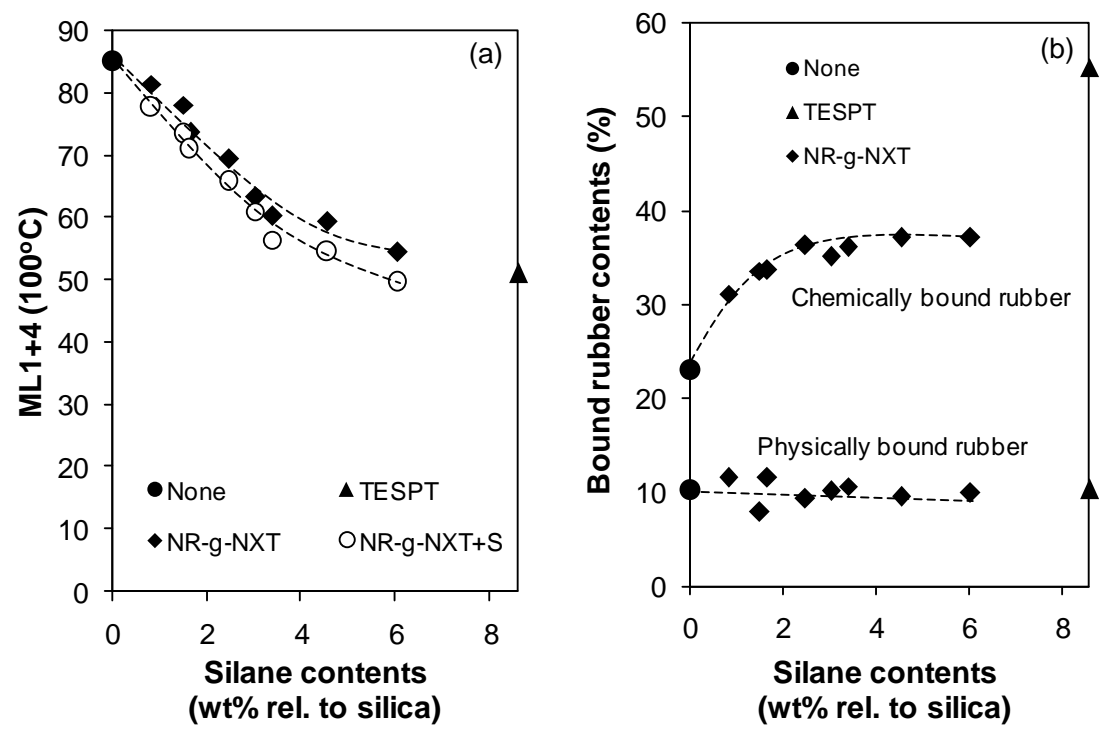

Figure 9.1 Mooney viscosity (a) and bound rubber contents (b) of silica-filled NR compounds with NXT-grafted-NR as compatibilizer without and with sulfur compensation, in comparison with the reference compounds with TESPT and without compatibilizer.

Figure 9.1(a) shows the Mooney viscosities of the silica-filled NR compounds with NXT-grafted-NR as compatibilizer without and with sulfur compensation in comparison with the reference compounds. Increasing amounts of NXT-silane in the grafted-NR significantly decrease the Mooney viscosities of both sets of silica-filled compounds without and with sulfur compensation, in which the sulfur-compensated ones show slightly lower viscosities. The compounds containing NR-g-NXT at $6.1 \mathrm{wt} \%$ silane relative to the silica show more or less the same level of the Mooney viscosity to that of the reference compound with $8.6 \mathrm{wt} \%$ TESPT relative to the silica.

The bound rubber content indicates the degree of silica-rubber interaction in the silica-filled rubber compounds. This measurement was done by using a non-productive compound (without curative) as obtained after the $1^{\text {st }}$ mixing step. The extra sulfur was added in the $2^{\text {nd }}$ step of mixing on the two roll mill and so there is only one set of data available. Chemically and physically bound rubber contents of the silica-filled NR compounds with different amounts of NXT-grafted-NR as compatibilizer are shown in Figure 9.1(b) in comparison with those of the reference compounds. As expected, the silica-filled NR compound with TESPT shows the highest chemically bound rubber content due to the strong filler-rubber interactions and some premature crosslinks generated by the sulfur atoms in the TESPT tetrasulfide structure. The silica-filled NR compound without 
any compatibilizer shows the lowest chemically bound rubber, which is somewhat higher than the value of its physically bound rubber content. In the non-compatibilized compound, the chemically bound rubber can be attributed to the trapped rubber within the tight fillerfiller network and some filler-rubber interactions enhanced by the naturally occurring polar functional groups such as aldehydes, proteins and phospholipids in the NR. The addition of NXT-grafted-NR as compatibilizer significantly increases the chemically bound rubber contents with increasing NXT amount to about $3 \mathrm{wt} \%$ in relation to the silica and thereafter the values level off. The compound with NXT-grafted-NR as compatibilizer contains less active groups to react and/or interact with the silanol groups on the silica surface. Moreover, it has no contribution from partial network formation like for the TESPT compound. The chemically bound rubber content of the system with NR-g-NXT is therefore still significantly lower than the reference compound with TESPT. However, the physically bound rubber contents of all the silica-filled NR compounds are comparable.
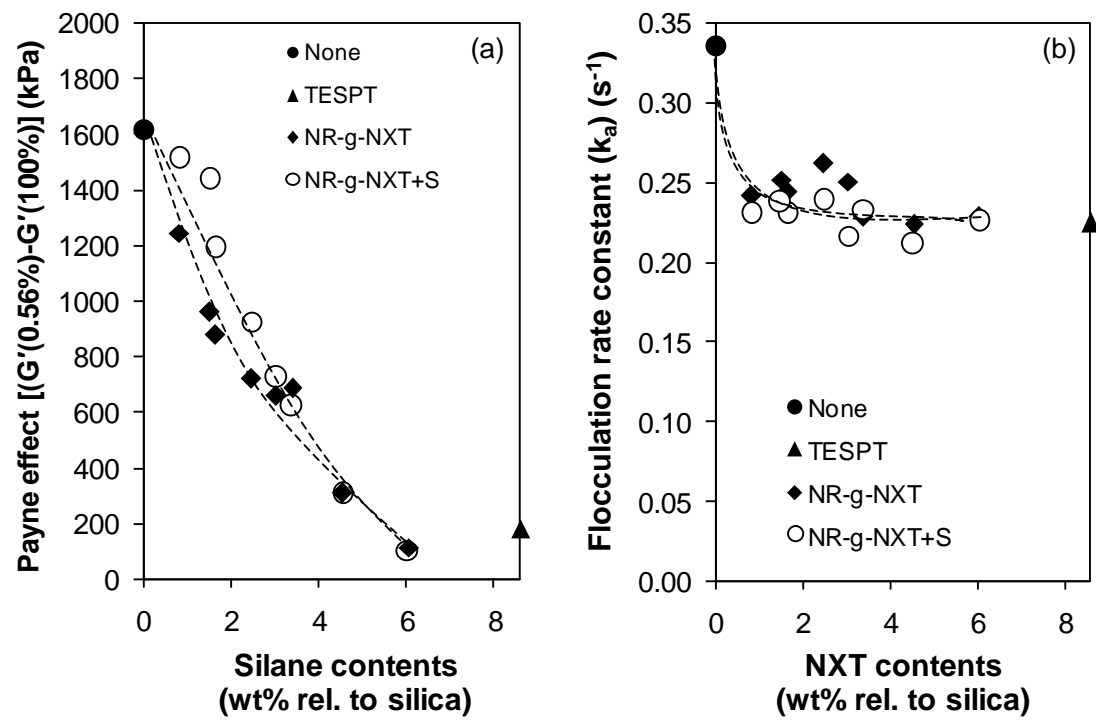

Figure 9.2 Payne effect (a) and flocculation rate constant (b) of silica-filled-NR compounds with NXT-grafted-NR as compatibilizer without and with sulfur compensation, in comparison with reference compounds with TESPT and without compatibilizer.

The Payne effect of the silica-filled NR compounds dramatically decreases with the addition of NXT-grafted-NR as compatibilizer as shown in Figure 9.2(a), however, there is no significant difference between the silica-filled compounds without and with sulfur compensation. The increasing silane contents in the silica-filled compounds 
increase the amount of reactive groups to interact or react with the silanol groups on the silica surface which leads to a decrease of silica surface polarity and silica agglomeration. The Payne effects of the silica-filled compounds decrease to a similar level as that of the silica-filled compounds with TESPT when NR-g-NXT at 4-6 wt\% silane relative to the silica was used.

The flocculation rate constant $\left(k_{a}\right)$ of the silica-filled NR compounds with NXTgrafted-NR with and without sulfur compensation is shown in Figure 9.2(b). As previously discussed in Chapter 8, the addition of NXT-grafted-NR reduces the flocculation rate due to a reduction of the silica polarity after the silanization. The compounds without sulfur compensation show a fluctuation of data that may be caused by experimental error, but the ones with sulfur compensation show a better trend with slightly lower values. The addition of just a small amount of NXT-grafted-NR decreases the flocculation rate constant to the same level of TESPT. The effective suppression of the silica reagglomeration is derived from the silanization of the grafted silane onto the silica surface as well as the shielding effect.

\subsubsection{Cure characteristics}
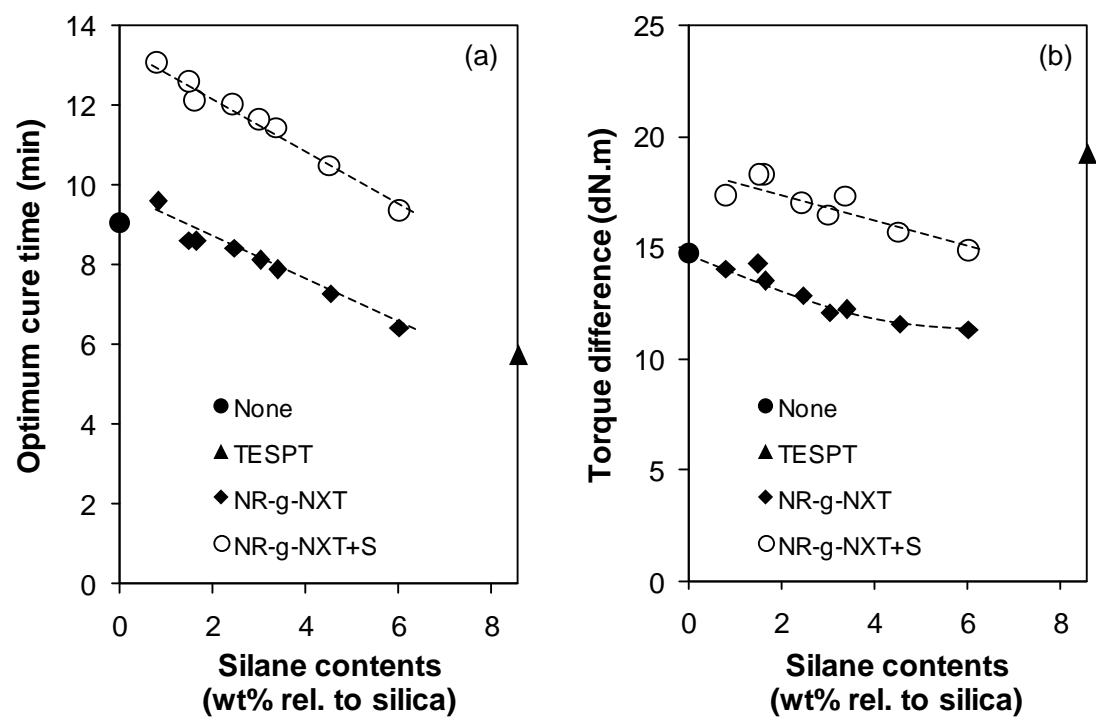

Figure 9.3 Optimum cure time (a) and torque difference (b) of silica-filled NR compounds with NXT-grafted-NR as compatibilizer without and with sulfur compensation, in comparison with the reference compounds with TESPT and without compatibilizer. 
Optimum cure time and torque difference of silica-filled NR compounds with NXT-grafted-NR as compatibilizer without and with sulfur compensation are shown in Figures 9.3(a) and (b) in comparison with those containing TESPT and without any compatibilizer. In Figure 9.3(a), the silica-filled NR-compound with TESPT shows the shortest optimum cure time as the silane has efficiently reacted with the silanol groups on the silica surface, leading to more hydrophobicity and less curative adsoption on the silica surface. Moreover, TESPT is a sulfur donor. ${ }^{[6]}$ The TESPT-compound therefore has a faster cure time compared to the other compounds. The optimum cure times of the silicafilled NR compounds with NXT-grafted-NR as compatibilizer decrease with increasing NXT-contents due to more reactive ethoxy-groups available to react with the silanol groups resulting in less interference with the curatives in the vulcanization reaction. The addition of more elemental sulfur to correct the total sulfur content towards the sulfur contained in the TESPT compound results in longer cure times, indicating that the change of sulfur-to-accelerator ratio has an influence on the crosslinking reaction in the rubber. In this case, the increase of sulfur content while the accelerator and activator contents remain the same leads to a longer time required to complete the vulcanization reaction.

The silica-filled NR-compounds with NXT-grafted-NR as compatibilizer show a decrease of torque difference with increasing amounts of NXT-silane and the compounds with sulfur correction show a higher torque difference compared to the compounds with normal sulfur content, as seen in Figure 9.3(b). When the amount of sulfur is increased, the crosslink density in the rubber matrix is increased. However, at the same sulfur content, the compound with NXT-grafted-NR has a lower torque difference compared to the compound with the use of straight TESPT, and the torque difference decreases with increasing NXT-content. This decreasing torque difference might be caused by the presence of deblocked NXT-fragments which can act as a plasticizer, as previously discussed in Chapter 6, but was not further studied in the present context. 


\subsubsection{Tensile properties and fractured surface topography}

\subsubsection{Tensile properties}
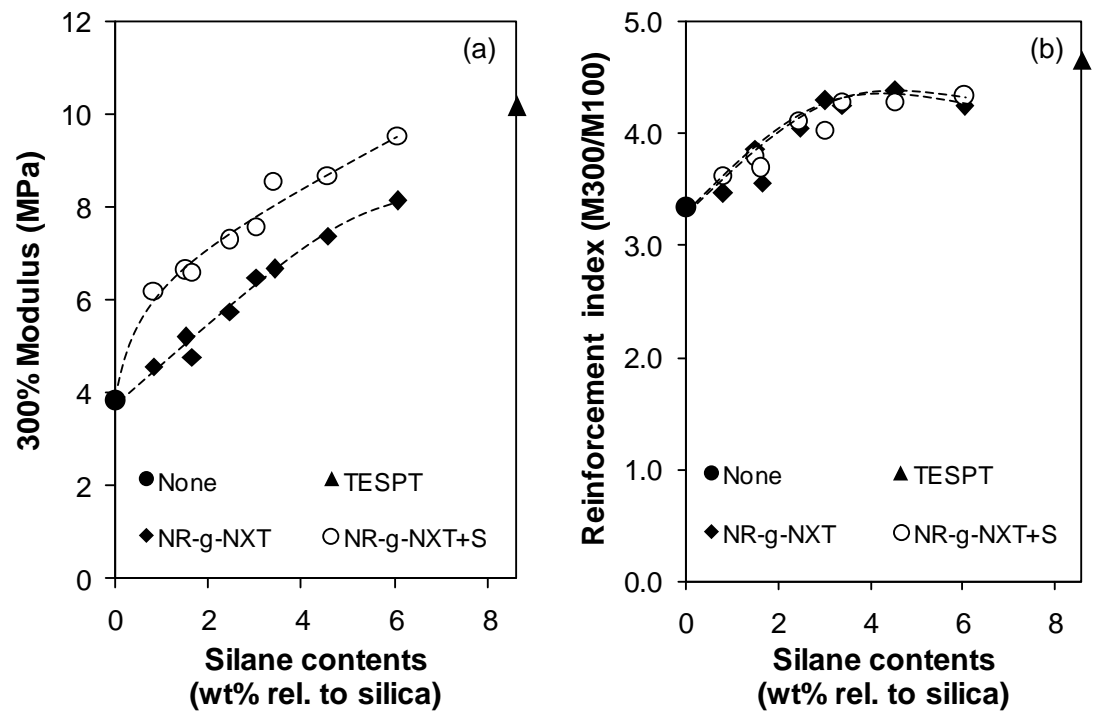

Figure 9.4 Modulus at 300\% elongation (a) and reinforcement index (b) of silica-filled NR compounds with NXT-grafted-NR as compatibilizer without and with sulfur compensation, in comparison with the reference compounds with TESPT and without compatibilizer.

The $300 \%$ Modulus and reinforcement index of the silica-filled NR-vulcanizates with NXT-grafted-NR as compatibilizer, with and without sulfur correction, increase with rising silane contents as shown in Figures 9.4(a) and (b). Sulfur compensation increases the modulus of the vulcanizates due to a resulting increase of the crosslink density via sulfur crosslinks as indicated by the increase of torque difference in Figure 9.3(b). However, the sulfur correction in the NXT-grafted-NR compatibilized vulcanizates has no effect on the reinforcement index, as seen in Figure 9.4(b). The decrease in torque difference with increasing NXT-grafted-NR contents in Figure 9.3(b) does not reflect in the $300 \%$ modulus, as would have been expected: on the contrary, the $300 \%$ modulus increases, as does the reinforcement index. The ultimate $300 \%$ modulus and reinforcement index for the sulfur compensated compounds almost reach the level obtained for straight TESPT. 

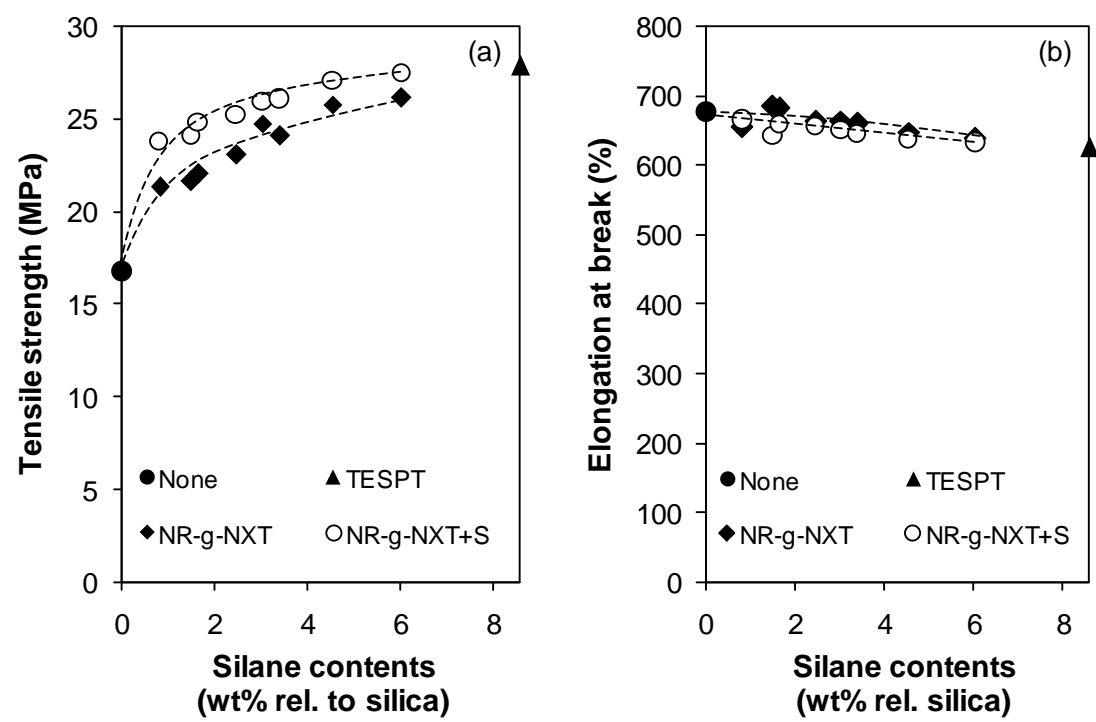

Figure 9.5 Tensile strength (a) and elongation at break (b) of silica-filled NR compounds with NXT-grafted-NR as compatibilizer without and with sulfur compensation, in comparison with the reference compounds with TESPT and without compatibilizer.

Tensile strength of the vulcanizates increases with increasing NXT-contents in the NXT-grafted-NR due to an increase of silica-rubber interactions in the compounds, and the sulfur correction increases the tensile strength of the vulcanizates relative to no sulfur-correction. The presence of 4.6 wt $\%$ NXT relative to the silica in the NXT-graftedNR used as compatibilizer combined with sulfur correction increases the tensile strength to the same level as that of the use of optimum TESPT content. With sulfur correction and increasing NXT content, the elongation at break of the vulcanizates tends to decrease slightly as a result of more filler-rubber interactions and a higher crosslink density. 


\subsubsection{Surface topography}
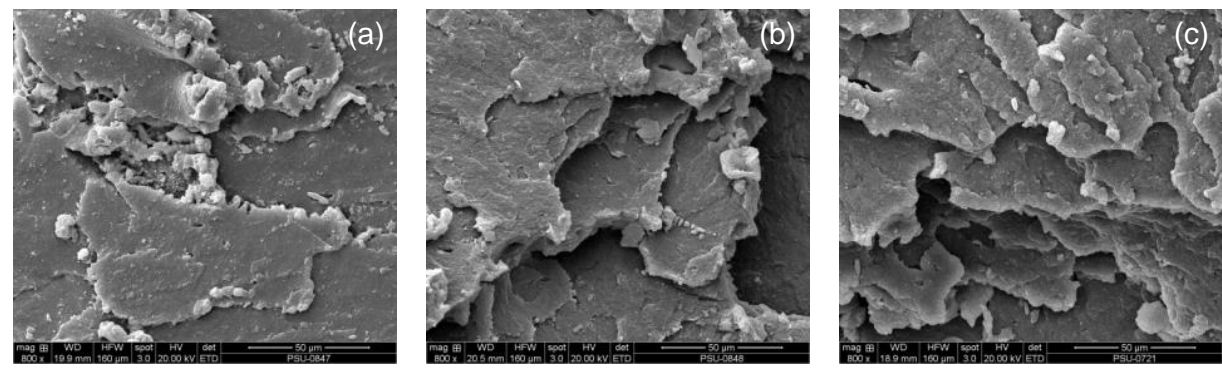

Figure 9.6 Tensile fractured surfaces of silica-filled NR vulcanizates: (a) with NR-g-NXT containing total $1.7 \mathrm{wt} \%$ NXT rel. to silica; (b) with NR-g-NXT containing total $1.7 \mathrm{wt} \%$ NXT rel. to silica and sulfur correction; (c) with 8.6 wt\% TESPT rel. to silica.

With elemental sulfur added into the compounds to compensate for the sulfur in the TESPT molecules, the fractured surfaces of the vulcanizate show more tear lines which correlates with their higher tensile strength: Figure 9.5.

\subsubsection{Dynamic mechanical properties}

Dynamic mechanical analysis has been applied to study the relaxation behavior of the filled polymer systems which correlates with the mobility of the polymer chains, that depends on the specific character of the filler and the polymer matrix, the amount of filler and degree of filler-polymer interactions. ${ }^{[7-9]}$ 


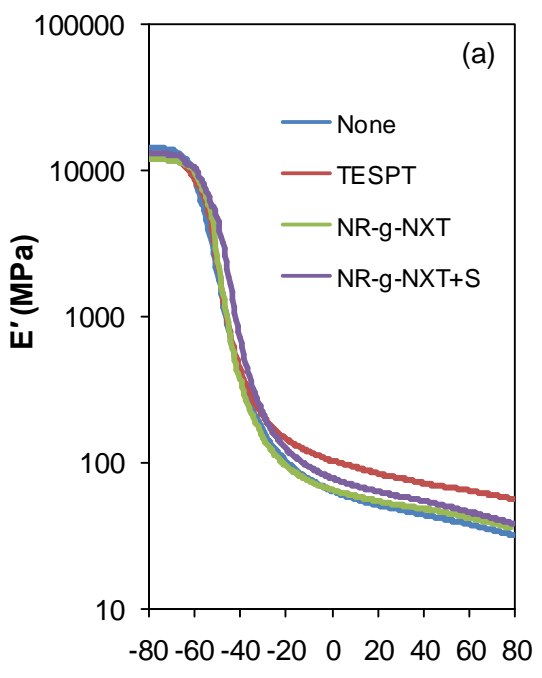

Temperature $\left({ }^{\circ} \mathrm{C}\right)$

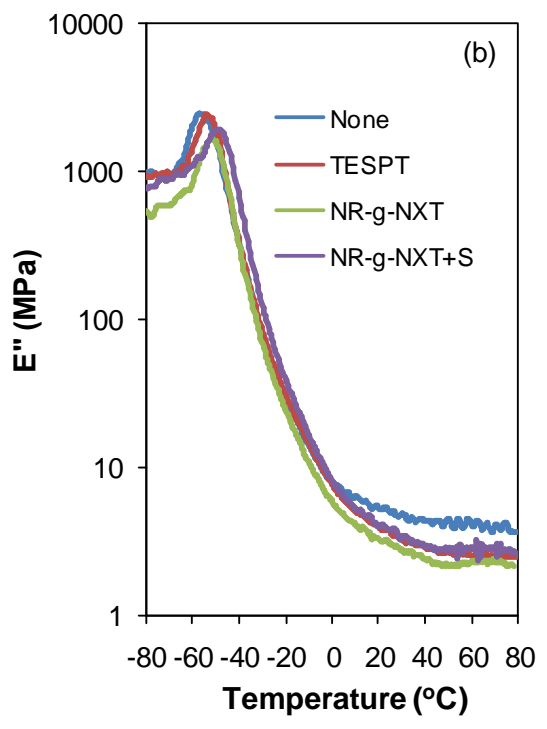

Figure 9.7 Storage (a) and loss (b) moduli of silica-filled NR compounds with NXT-graftedNR with 6.1 wt\% NXT rel. to silica as compatibilizer without and with sulfur compensation, in comparison with the reference compounds with TESPT and without compatibilizer.

Storage ( $\left.E^{\prime}\right)$ and loss (E") moduli of the silica-filled NR with NXT-grafted-NR, with and without sulfur correction, with TESPT and without compatibilizer are compared in Figures 9.7(a) and (b). In the glassy state, all the filled vulcanizates show a similar level of $E^{\prime}$ even though they have different degrees of filler-rubber interaction as reflected in the bound rubber contents in Figure 9.1(a). However, the E"-peak of the silica-filled NR with NXT-grafted-NR is shifted to a higher temperature compared to the silica-filled NR with TESPT and without compatibilizer, respectively. The increase of sulfur content due to the sulfur compensation relative to the TESPT compound results in a slight further shift of the E"-peak due to the increase of crosslink density, which restricts the segmental motion of the rubber chains. In the rubbery region, the silica-filled NR vulcanizate with TESPT shows the highest $E^{\prime}$ due to the greatest extent of filler-rubber interactions and an increase of crosslink points through silane bridges, but lower $E^{\prime \prime}$ compared to the noncompatibilized system. The pronounced silica-silica network and trapped rubber chains in the silica-filled NR vulcanizate without compatibilizer results in the highest loss of energy. 


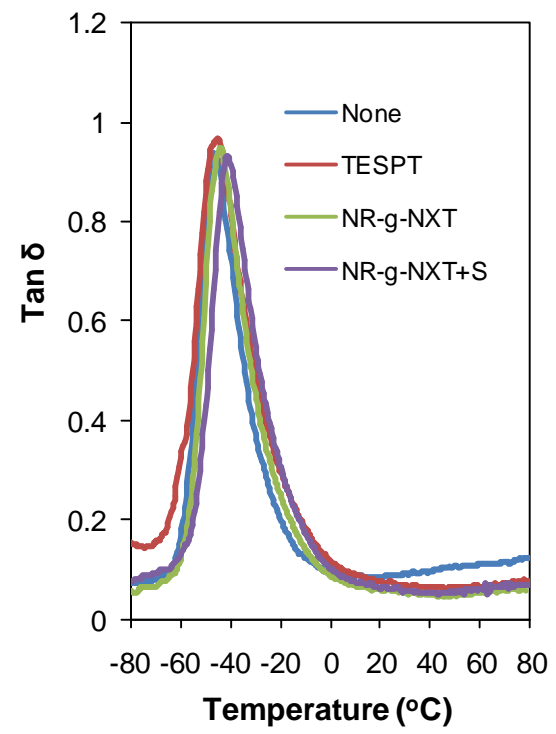

Figure 9.8 Tan $\delta$ of silica-filled NR compounds with NXT-grafted-NR with 6.1 wt\% NXT rel. to silica as compatibilizer without and with sulfur compensation, in comparison with the reference compounds with TESPT and without compatibilizer.

Tan $\delta$ of silica-filled NR vulcanizates with NXT-grafted-NR as compatibilizer with and without sulfur compensation is shown in Figure 9.8 in comparison with the vulcanizates without any compatibilizer and with TESPT. As previously observed in Figure 8.16, the addition of NXT-grafted-NR as compatibilizer into the silica-filled NR slightly shifts the $T_{g}$ 's of the vulcanizates to higher temperature. With sulfur compensation the $T_{g}$ further shifts as a result of the increased crosslink density. The tan $\delta$ value at peak is also reduced after the sulfur compensation as more crosslink points restrict the relaxation and reduce chain flexibility. The tan $\delta$ values at different positions are summarized in Table 9.3. 
Table 9.3 $\mathrm{T}_{\mathrm{g}}$ and tan $\delta$ values of silica-filled NR vulcanizates with different compatibilizers.

\begin{tabular}{lccccc}
\hline \multirow{2}{*}{\multicolumn{1}{c}{ Compatibilizer types }} & \multicolumn{2}{c}{$\mathrm{T}_{\mathbf{g}}\left({ }^{\circ} \mathrm{C}\right)$} & \multicolumn{3}{c}{ Values of Tan $\delta$} \\
\cline { 2 - 6 } & $\begin{array}{c}\text { at } \tan \delta \\
\text { peak }\end{array}$ & $\begin{array}{c}\text { at } \mathrm{E}^{\prime \prime} \\
\text { peak }\end{array}$ & at peak & at $5{ }^{\circ} \mathrm{C}$ & at $60^{\circ} \mathrm{C}$ \\
\hline Without compatibilizer & -47 & -58 & 0.94 & 0.09 & 0.11 \\
TESPT & -45 & -54 & 0.97 & 0.10 & 0.07 \\
NR-g-NXT with NXT 1.7 wt\% & -44 & -52 & 0.96 & 0.07 & 0.05 \\
NR-g-NXT with NXT 3.4 wt\% & -44 & -52 & 0.96 & 0.07 & 0.05 \\
NR-g-NXT with NXT 6.1 wt\% & -44 & -52 & 0.95 & 0.08 & 0.05 \\
\hline NR-g-NXT with NXT 1.7 wt\%+S & -42 & -50 & 0.91 & 0.07 & 0.05 \\
NR-g-NXT with NXT 3.4 wt\%+S & -42 & -50 & 0.92 & 0.08 & 0.05 \\
NR-g-NXT with NXT 6.1 wt\%+S & -42 & -50 & 0.93 & 0.08 & 0.06 \\
\hline
\end{tabular}

The use of TESPT in the silica-filled NR vulcanizate gives the highest $\tan \delta$ at $5^{\circ} \mathrm{C}$, that suggests the best wet grip for a tire-tread made thereof; and significantly lower $\tan \delta$ at $60^{\circ} \mathrm{C}$ compared to the non-compatibilized vulcanizate. However, the tan $\delta$ at $60^{\circ} \mathrm{C}$ of the silica/TESPT system is slightly higher when compared to the vulcanizate containing NR-g-NXT. The use of NR-g-NXT as compatibilizer suggests therefore somewhat inferior tire wet grip compared to the use of TESPT, but the lowest tan $\delta$ values at $60^{\circ} \mathrm{C}$ imply the lowest tire rolling resistance. The addition of sulfur compensation relative to the reference compound with TESPT has no influence on tan $\delta$ at both $5^{\circ} \mathrm{C}$ and $60^{\circ} \mathrm{C}$.

\subsection{Conclusions}

The properties of silica-filled NR compounds with NXT-grafted-NR as compatibilizer without and with sulfur compensation change according to the NXT amounts. The sulfur-compensated-compounds show slightly lower Mooney viscosities, comparable Payne effect and flocculation rate constant, and significantly higher cure torque difference compared to the counterparts without sulfur compensation. Compared to the straight use of 8.6 wt\% TESPT, the addition of NR-g-NXT with 4.5-6 wt\% NXT relative to the silica, either with or without sulfur compensation, gives more or less the same levels of Mooney viscosity and Payne effect while with about $1 \mathrm{wt} \%$ NXT relative to the silica already gives a similar level of the flocculation rate constant. Sulfur compensation increases the modulus and tensile strength of the vulcanizates due to the increased crosslink density, but has no effect on the reinforcement index. The $300 \%$ modulus, reinforcement index, tensile strength and elongation at break for the sulfur compensated compounds reach the level obtained for straight TESPT. The presence of NR-g-NXT as 
compatibilizer results in a shift of the glass transition temperature $\left(T_{g}\right)$ to a higher temperature compared to the silica-filled NR with TESPT and without compatibilizer. With sulfur compensation, the $\mathrm{T}_{\mathrm{g}}$ is slightly shifted further due to the increase in crosslink density. As far as can be derived from the tan $\delta$ values at $5^{\circ} \mathrm{C}$ and $60^{\circ} \mathrm{C}$, the use of NXTgrafted-NR as compatibilizer suggests somewhat inferior tire wet grip but superior tire rolling resistance compared to the use of TESPT, and the sulfur compensation shows no influence on the tan $\delta$ values at both temperatures.

Overall the use of NXT-grafted-NR with about $6 \mathrm{wt} \%$ NXT relative to the silica and with sulfur compensation gives the properties of compounds and vulcanizates; i.e. Mooney viscosity, Payne effect, 300\% modulus, reinforcement index, tensile strength, elongation at break, storage modulus and $\tan \delta$ at $60^{\circ} \mathrm{C}$ that reach the levels obtained for straight use of TESPT at $8.6 \mathrm{wt} \%$ relative to the silica. Therefore, the NXT-grafted-NR with sulfur compensation is an alternative to the conventional TESPT for the silica-reinforced NR compounds, providing better environmental friendliness and safety due to less emitted ethanol. The amounts of $8.6 \mathrm{wt} \%$ TESPT and $6 \mathrm{wt} \%$ NXT relative to the silica are more or less equal in moles, and so the compounds with NXT emit half the ethanol compared to the use of TESPT. Moreover, the NR-g-NXT can be prepared by melt mixing and the preprepared grafted material is easier to use than the straight silane in liquid form.

\subsection{References}

1. P. Saramolee, K. Sahakaro, N. Lopattananon, W.K. Dierkes, J.W.M. Noordermeer, Adv. Mat. Res., 844, 235 (2014).

2. J.W. ten Brinke, P.J. van Swaaij, L.A.E.M. Reuvekamp, J.W.M. Noordermeer, Rubber Chem. Technol., 76, 12 (2003).

3. J.W. ten Brinke, P.J. van Swaaij, L.A.E.M. Reuvekamp, J.W.M. Noordermeer, Comp. Sci. Technol., 63, 1165 (2003).

4. K. Kaewsakul, W.K. Dierkes, J.W.M. Noordermeer, S. Sahakaro, Kautsch. Gummi Kunstst., 65, 33 (2014).

5. S. Wolff, M.-J. Wang, Rubber Chem. Technol., 65, 329 (1992).

6. S. Wolff, Kautsch. Gummi Kunstst., 30, 516 (1977).

7. C.G. Robertson, C.J. Lin, M. Rackaitis, C.M. Roland, Macromolecules, 41, 2727 (2008).

8. L. Ladouce-Stelandre, Y. Bomal, L. Flandin, D. Labarre, Rubber Chem. Technol., 76, 145 (2003).

9. V. Arrighi, I.J. McEwen, H. Qian, M.B. Serrano Prieto, Polymer, 44, 6259 (2003). 


\section{Summary}

Silica has conventionally been used to produce light-colored rubber products and mechanical goods, but is increasingly used in tire tread compounds to reduce rolling resistance and thus automotive fuel consumption. However, its use is hampered by a large number of silanol groups on the silica surface that lead to strong filler-filler bonds and poor interaction with non-polar rubbers. Silane coupling agents have been practically used in silica-filled compounds to improve silica dispersion and the interactions between silica and rubber. The mixing conditions must be optimized to achieve a good level of silanization and thus properties. A whole or partial replacement of silane coupling agents with chemically functionalized natural rubber (NR) as a compatibilizer should reduce the complications arising from the silanization reaction. This will lead to more use of silica compounds for tires and consequently more efficient use of automotive energy. After an introduction in Chapter 1, a literature overview with emphasis on the reinforcement efficiency of silica-filled rubber with different compatibilizing systems is given in Chapter 2. Filler characteristics, filler reinforcement in general, the development of silica-silane technology and alternative approaches to replace the silane are addressed. Particular attention in the present thesis is given to the use of chemically modified NR as compatibilizer for silica-reinforced NR compounds. The experimental part starts with investigating the effect of epoxidized natural rubber (ENR) with different epoxy contents on the reinforcement of silica-filled NR as detailed in Chapter 3. The use of ENR as compatibilizer improves the filler-rubber interaction, silica dispersion and tensile strength. Increase of the ENR content and mole\% of epoxide groups decreases the filler-filler interaction and improves silica dispersion, as reflected in a lower Mooney viscosity, Payne effect, flocculation rate constant and filler networking factor. The addition of ENR-38 and ENR-51 (with 38 resp. 51 mole\% epoxide) leads to higher filler-rubber interaction parameters, chemically bound rubber, tensile strength and silica dispersion levels. With respect to tire performance, the presence of ENR-38 and ENR-51 as compatibilizer improves wet grip but negatively affects rolling resistance due to an increased glass transition temperature of the epoxidized NR. The addition of ENR-51 at 7.5 phr shows the best overall properties, however still somewhat lower when compared to a compound with the commonly employed bis-(triethoxysilylpropyl)tetrasulfide (TESPT) silane as coupling agent. 
The use of ENR-51 was optimized at $7.5 \mathrm{phr}$, but without additional silane coupling agent the properties of the silica-filled NR are still inferior to the use of TESPT alone. For further property improvement, the ENR is used in combination with TESPT and the results are discussed in Chapter 4. The use of ENR-51 at $7.5 \mathrm{phr}$ in combination with TESPT at 2 wt\% relative to the silica further decreases the Mooney viscosity, Payne effect, flocculation rate constant and increases bound rubber contents of the silica-filled NR compounds, when compared to a silica-filled compound with ENR-51 alone. A further increase of TESPT-content has only a small additional effect on the compound properties. Tensile strength and modulus of the vulcanizates increase with increasing TESPTcontents and further improve by sulfur compensation relative to the reference compound with TESPT, to equilibrate the level of crosslink density. The use of ENR-51 at $7.5 \mathrm{phr}$ with TESPT at 4 wt\% relative to silica and sulfur compensation gives vulcanizates with the same levels of tensile strength compared to vulcanizates with TESPT at $8.6 \mathrm{wt} \%$ relative to silica. The presence of ENR in the silica-filled NR affects the dynamic mechanical properties. Above the glass-to-rubber transition, the ENR-containing vulcanizates show a higher $\tan \delta$ compared to the silica/TESPT system. The addition of TESPT to the ENRcompatibilized compounds causes only a small change in $\mathrm{T}_{\mathrm{g}}$ and $\tan \delta$ at $5^{\circ} \mathrm{C}$, but lowers $\tan \delta$ at $60^{\circ} \mathrm{C}$. Furthermore, the addition of extra sulfur leads to an increase of $\mathrm{T}_{\mathrm{g}}$ and lower $\tan \delta$ at both $5^{\circ} \mathrm{C}$ and $60^{\circ} \mathrm{C}$ as a result of increased crosslink density. SEM micrographs clearly show good silica dispersion in all compatiblized vulcanizates, and the tensile fracture surfaces of the materials show different tear line patterns dependent on their tensile strengths.

The use of three compounds with ENR-51 at $7.5 \mathrm{phr}$ in combination with three types of silane: TESPT, 3-octanoylthio-1-propyltriethoxysilane (NXT) and 3mercaptopropyl-di(tridecan-1-oxy-13-penta(ethyleneoxide))ethoxysilane (VP Si-363) in the range of 2 to $5 \mathrm{wt} \%$ relative to the silica, is comparatively discussed in Chapter $\mathbf{5}$. Addition of silane reduces the compound viscosity slightly but clearly enhances the chemically bound rubber content and cure properties. Among the 3 different ENR-51/silane combinations, the use of ENR/TESPT gives the highest chemically bound rubber content and tensile properties, whereas the ENR/NXT combination gives higher chemically bound rubber content but similar tensile properties compared to the ENR/VP Si-363 system. The better improvement of the overall properties by the use of TESPT over the other two types of silanes is due to the bis-(triethoxysilyl)- and tetrasulfide-functional groups that provide more ethoxy groups relatively to react with the silanol groups on the silica surface, and the reactive free sulfur released by TESPT to involve in network formation. When the amount 
of silane coupling agent is fixed at $4.0 \mathrm{wt} \%$ relative to silica in combination with $7.5 \mathrm{phr}$ of ENR-51 as compatibilizers in the silica-filled NR compound, the addition of extra sulfur to correct the total sulfur content relative to the reference compound with TESPT leads to increases of modulus and tensile strength while elongation at break decreases. The use of an ENR/silane combination increases the tan $\delta$ at $5^{\circ} \mathrm{C}$ and reduces the tan $\delta$ at $60^{\circ} \mathrm{C}$ compared to the use of ENR-51 alone, indicating an improvement in wet traction and rolling resistance of tires made thereof. However, overall the use of TESPT alone at optimum loading still gives the best mechanical and dynamic mechanical properties compared to the presence of ENR as compatibilizer.

Among the three types of silanes studied in combination with ENR-51 and sulfur compensation, the use of 2.2 phr TESPT together with 7.5 phr ENR-51 provides the best overall properties, but it does still not fully match the properties of a compound with TESPT alone at optimal quantity, i.e. $4.7 \mathrm{phr}$ as previously observed in Chapter 4. However, in the perspective of the "Magic Triangle of Tire Technology" where wet skid resistance, rolling resistance and wear are all to be optimized, the combination of ENR-51, TESPT and sulfur compensation may become a better option for "Winter Tires" that demand good wet grip, providing that real tire tests have yet to confirm this.

By excluding the ENR as compatibilizer, the properties of silica-fillled NR with four types of silane coupling agents, i.e. TESPT, NXT, VP Si-363 and vinyltriethoxysilane (VTES) is investigated in order to screen for a potential silane coupling agent to be used later for grafting onto NR molecules, as detailed in Chapter 6. Among the four silane types, VTES gives the poorest overall properties due to its poor shielding effect, and the vinyl-group that is difficult to couple with NR rubber chains in the presence of sulfur. Hence, VTES is not an effective silane coupling agent for this silica-filled NR system. The use of NXT and VP Si-363 shows a good improvement in compound properties, i.e. mixing energy, Mooney viscosity, cure behaviors, bound rubber contents, and an increase of mechanical and dynamic mechanical properties compared to that without silane, but the properties are inferior to the use of TESPT, mainly due to lower crosslink densities compared with TESPT which has an intrinsic "sulfur-donating" effect. This was not further pursued in the context of this chapter. The incorporation of NXT silane provides better scorch safety, and use at the same amount of ethoxy-functional groups with reference to TESPT gives vulcanizates with the same level of tensile strength. With respect to tire performance, NXT offers better properties than VP Si-363 but still somewhat inferior when compared to the reference compound with TESPT.

Later on, NXT and VP Si-363 silane coupling agents are employed to graft onto $\mathrm{NR}$ in order to introduce reactive side-groups for interacting and/or reacting with the 
silanol groups on the silica surface. Chapter 7 shows that the silane-grafted-natural rubbers are successfully prepared under melt mixing conditions in an internal mixer at $140^{\circ} \mathrm{C}$ using $0.1 \mathrm{phr}$ of $1,1^{\prime}$-di(tert-butylperoxy)-3,3,5-trimethylcyclohexane or Luperox® $231 X L 40$ as initiator. By elemental analysis via the SEM-EDX technique, the modified NRs contain silicon (Si) atoms in their structures, and the amounts of oxygen and silicon in the grafted NRs increase with increasing amounts of silane used for the reaction. Both VP Si363-grafted-NR and NXT-grafted-NR show infrared absorption bands at 3270, 1075 and $1035 \mathrm{~cm}^{-1}$ assigned to the deformations of $-\mathrm{OH}$, Si-O-C and Si-O-Si, respectively, and the ${ }^{1} \mathrm{H}$ NMR absorption band at $3.8 \mathrm{ppm}$ assigned to the methylene proton of the ethoxy groups.

The use of silane-grafted-NRs as compatibilizers in silica-filled NR compounds is discussed in Chapter 8 in comparison with the straight use of silane in the compound formulation and the use of TESPT at its optimal amount. The increasing amount of NXTand VP Si-363-grafted-NRs as compatibilizers in silica-filled NR compounds decreases the Mooney viscosity, filler-filler interaction or Payne effect, flocculation rate constant and increases the chemically bound rubber contents, $300 \%$ modulus, reinforcement index and tensile strength. The reinforcement index and tensile strength of the silica-filled NR vulcanizates almost level off when the silane content in the silane-grafted-NRs, which includes free and grafted silane, exceeds $3.0 \mathrm{wt} \%$ relative to the silica. Tensile fractured surfaces of the silica-filled NR vulcanizates indicate ductile failure with surface roughness and many tear lines when the silane-grafted-NRs are used, like the case for the compound with TESPT. At the same silane loading, the use of silane-grafted-NR as compatibilizer gives a better improvement in Payne effect, chemically bound rubber content, $300 \%$ modulus and tensile strength compared to the straight use of that silane. The presence of NXT provides better improvement of overall properties than VP Si-363, but both are still lower than the use of TESPT at its optimum content. In the presence of the silane-grafted-NR as compatibilizer, the $T_{g}$ of the silica-filled NR vulcanizates is shifted to a higher temperature. The tan $\delta$ at $5^{\circ} \mathrm{C}$ that relates to wet grip is slightly decreased but the tan $\delta$ at $60^{\circ} \mathrm{C}$ is clearly reduced indicating a lower tire rolling resistance, compared to the reference compound with TESPT. Sulfur compensation for the compounds having NXT-grafted-NR as compatibilizer is also investigated to enhance the properties of the silica-filled NR as discussed in Chapter 9. The sulfur-compensated-compounds show slightly lower Mooney viscosity but significantly higher cure torque difference compared to the compound without extra sulfur. With sulfur compensation relative to the sulfur contained in the reference TESPT compound, the use of NR-g-NXT with 6 wt\% NXT relative to the silica reduces Mooney viscosity and Payne effect as well as enhances the 
$300 \%$ modulus and tensile strength of the silica-filled NR to almost the same level as obtained from the straight use of TESPT at its optimum content, but without significant effects on the tan $\delta$ values at 5 and $60^{\circ} \mathrm{C}$. As the amounts of $8.6 \mathrm{wt} \%$ TESPT and $6 \mathrm{wt} \%$ NXT relative to the silica are more or less equal in molar amounts, the use of NXT-graftedNR therefore emits only a half the ethanol commonly produced by TESPT, leading to a better environmentally friendly and safe system.

A growing importance of silica-reinforced tire compounds for reduction of automotive fuel consumption motivates research and development of the relevant rubber technology. In addition to the commonly used silica/silane technology, alternative compatibilizers to totally or partially replace the silane for more "green" products are of interest. This present thesis provides an in-depth study on the application of ENRs as alternative compatibilizer. The use of ENRs alone as compatibilizers clearly shows an enhancement already of the silica-NR compatibility, silica dispersion and some mechanical properties. The properties can be further enhanced by using ENR in combination with only small amounts of silane coupling agents and sulfur compensation relative to a reference compound with TESPT. In addition to the more general use of ENRs, silane-grafted-NR offers another alternative. The preparation of silane-grafted-NR in the melt mixing state is practical but complicated. The non-purified product contains both free and grafted silane. Nevertheless, the use of non-purified silane-grafted NRs as compatibilizers shows an improvement in filler-rubber interactions and the properties of silica-reinforced NR. At the same total silane content, the use of silane-grafted-NR gives overall better properties than the straight use of silane and the properties can be further enhanced by sulfur compensation, taking the compound with optimal content of TESPT as reference. Overall, even though the application of chemically modified NRs in the forms of ENRs and silane-grafted-NR as compatibilizers without further silanes results in inferior overall properties compared to the use of the state-of-the-art TESPT, the right combination of modified NR with only small amounts of TESPT and sulfur compensation offers an alternative system with the advantages of NR-based product usage and less ethanol emission from the silanization reaction. Moreover, the use of ENR brings in a good wet grip in the perspective of the "Magic Triangle of Tire Technology". 


\section{Samenvatting}

Silica wordt van oudsher gebruikt om licht-gekleurde rubber producten en mechanische artikelen te maken, maar wordt in toenemende mate gebruikt in autobanden loopvlak-mengsels om de rolweerstand te verlagen en daardoor het brandstofverbruik. Evenwel, het gebruik daarvan wordt bemoeilijkt door het grote aantal silanol-groepen op het silica-oppervlak, hetgeen leidt tot sterke vulstof-vulstof bindingen en slechte interactie met niet-polaire rubbers. Silaancouplingagents worden in de praktijk ingezet in silicagevulde mengsels om de silica-dispersie en de interacties tussen silica en rubber te verbeteren. De mengcondities moeten worden geoptimaliseerd om een goed silanisatieniveau te bereiken en daarmee goede eigenschappen. Een algehele of gedeeltelijke vervanging van silaancouplingagents met chemisch gefunctionaliseerde Natuur Rubber (NR) als compatibilizer zou de complicaties van de silaniserings-reactie moeten verminderen. Dit zal dan leiden tot een hoger gebruik van silica mengsels voor banden en dientengevolge een efficiënter gebruik van de energie benodigd voor wegtransport. $\mathrm{Na}$ een inleiding in Hoofdstuk 1, wordt in Hoofdstuk 2 een literatuur overzicht gegeven met nadruk op de efficientie van de versterkende werking van silica-gevulde rubber met verschillende compatibiliserende systemen. Vulstof karakteristieken, vulstof-versterking in het algemeen, de ontwikkeling van de silica-silaan technologie en alternatieven ter vervanging van silaan worden belicht. Bijzondere aandacht in het huidige proefschrift ligt op het gebruik van chemisch gemodificeerde NR als compatibilizer voor silica-versterkte NR mengsels. Het experimentele gedeelte begint met een onderzoek naar het effect van geëpoxideerde natuurrubber (ENR) met verschillende epoxy-gehaltes, op de versterking van silica-gevulde NR, zoals gegeven in Hoofdstuk 3. Het gebruik van ENR als compatibilizer verbetert de vulstof-rubber interactie, de silica dispersie en de treksterkte. Verhogen van het ENR-gehalte en mol\% epoxide-groepen verlaagt de vulstof-vulstof interactie en verbetert de silica-dispersie, zoals blijkt uit de Mooney viscositeit, het Payne effect, de snelheidsconstante van uitvlokken van de silica en de vulstof netwerk-factor. Toevoeging van ENR-38 en ENR-51 ( met 38, resp. 51 mol\% epoxide) leidt tot hogere vulstof-rubber interactie parameters, chemische bound rubber, treksterkte en silicadispersie niveaus. Met betrekking tot banden-gedrag verbetert de aanwezigheid van ENR38 en ENR-51 als compatibilizers de natte grip, maar verslechtert de rolweerstand vanwege een verhoogde glasovergangs-temperatuur van de geëpoxideerde NR. De toevoeging van 7,5 phr ENR-51 geeft over het geheel genomen de beste eigenschappen, 
maar nog wel enigszins lager in vergelijking met een mengsel met het gewoonlijk gebruikte bis-(triethoxysilylpropyl)tetrasulfide (TESPT) silaan als coupling agent.

Het gebruik van ENR-51 werd geoptimaliseerd op 7,5 phr, maar zonder extra silaancoupling agent blijven de eigenschappen van silica-gevulde NR toch achter bij het gebruik van TESPT alleen. Voor verder eigenschappen-verbetering wordt het gebruik van ENR in combinatie met TESPT en de resultaten daarvan in Hoofdstuk 4 besproken. Gebruik van 7,5 phr ENR-51 in combinatie met 2 gew.\% TESPTbetrokken op de silica, verlaagt de Mooney viscositeit verder, evenals het Payne effect en de uitvlokkings snelheidsconstante, en verhoogt de bound rubber gehaltes van de silica-gevulde NR mengsels, in vergelijking met een silica-gevuld mengsel met alleen ENR-51. Een verdere verhoging van het TESPT-gehalte heeft slechts een klein additioneel effect op de mengsel-eigenschappen. De treksterkte en modulus van de vulcanisaten nemen toe met hogere TESPT-gehaltes en nog verder door zwavel-compensatie t.o.v. het referentiemengsel met TESPT, om de crosslink-dichtheid gelijk te trekken. Het gebruik van 7,5 phr ENR-51 samen met 4 gew\% TESPT betrokken op de silica, en zwavel compensatie geeft vulcanisaten met dezelfde niveaus treksterkte vergeleken met vulcanisaten met 8,6 gew\% TESPT betrokken op de silica. De aanwezigheid van ENR in de silica-gevulde NR beïnvloedt de dynamisch mechanische eigenschappen. Boven de glas-rubber overgang vertonen de ENR-bevattende vulcanisaten een hogere tan סvergeleken met het silica/TESPT systeem. Toevoeging van TESPT aan de ENR-gecompatibiliseerde mengsels veroorzaakt slechts een geringe verandering in $\mathrm{T}_{\mathrm{g}}$ en tan $\delta$ bij zowel $5{ }^{\circ} \mathrm{C}$ en 60 ${ }^{\circ}$ Ctengevolge van een verhoogde crosslink-dichtheid. SEM microscopische opnamen tonen duidelijk een goede silica dispersie in alle gecompatibiliseerdevulcanisaten, en de breukvlakken bij trekken van de materialen tonen verschillende patronen van scheur-lijnen afhankelijk van hun treksterktes.

Drie mengsels met ENR-51 in combinatie met drie types silaan: TESPT, 3octanoylthio-1-propyltriethoxysilaan (NXT) en 3-mercaptopropyl-di(tridecaan-1-oxy-13penta(ethyleenoxide))ethoxysilaan (VP Si-363) in de range van $2-5$ gew\% betrokken op de silica, worden vergeleken in Hoofdstuk 5. Toevoegen van silaan reduceert de mengsel viscositeit enigszins, maar verbetert duidelijk het chemische bound rubber gehalte en de crosslinking/vernettings eigenschappen. Van de 3 verschillende ENR51/silaan combinaties geeft ENR/TESPT het hoogste chemischebound rubber gehalte en trek-rek eigenschappen, terwijl de ENR/NXT combinatie weliswaar een hoger chemisch bound rubber gehalte geeft maar vergelijkbare trek-rek eigenschappen als het ENR/VP Si363 systeem. De betere eigenschappen door gebruik van TESPT vergeleken met de andere twee types silaan valt terug te voeren op de bis-(triethoxysilyl)- en tetrasulfide- 
functionele groepen, die relatief meer ethoxy-groepen aanbieden voor reactie met de silanol-groepen op het silica-oppervlak, en de reactieve vrije zwavel vrijgemaakt uit de TESPT die deelneemt in de netwerk-vorming. Bij een vaste hoeveelheid silaancoupling agent van 4,0 gew\% betrokken op de silica, in combinatie met 7,5 phr ENR-51 als compatibilizers in het silica-gevulde NR mengsel, leidt toevoegen van extra zwavel om te corrigeren voor de totale zwavel-hoeveelheid ten opzichte van het referentie-mengsel met TESPT tot verhoging van de molulus en trek-sterkte, terwijl de rek-bij-breuk afneemt. Het gebruik van een ENR/silaan verhoogt de tan $\delta$ bij $5{ }^{\circ} \mathrm{C}$ en verlaagt de tan $\delta$ bij $60{ }^{\circ} \mathrm{C}$ vergeleken met gebruik van alleen ENR-51, hetgeen een verbetering in natte tractie en rolweerstand indiceert van banden gebaseerd op deze combinatie. Echter over het geheel genomen geeft gebruik van een optimale dosering van alleen TESPT nog de beste dynamisch mechanische eigenschappen vergeleken met de aanwezigheid van ENR als compatibilizer.

Van de drie types bestudeerde silanen in combinatie met ENR-51 en zwavel compensatie, geeft gebruik van 2,2 phr TESPT samen met 7,5 phr ENR-51 de beste overall eigenschappen, maar evenaart nog niet volledig de eigenschappen van een mengsel met de optimale hoeveelheid van alleen TESPT: 4,7 phr, zoals eerder gezien in hoofdstuk 4. Evenwel, in het licht van de "Magische Driehoek van de Banden Technologie", waarin natte slipweerstand, rolweerstand en slijtage-weerstand alle moeten worden geoptimaliseerd, kan de combinatie van ENR-51, TESPT en zwavel compensatie een beter optie zijn voor "Winterbanden", die een goede natte grip vereisen, ofschoon echte banden-testen dat nog wel moeten bevestigen.

Met uitsluiting van ENR als compatibilizer worden de eigenschappen van silicagevulde NR met vier types silaancouplingagents, t.w. TESPT, NXT, VP Si-363 en vinyltriethoxysilaan (VTES) onderzocht teneinde een mogelijke silaancoupling agent uit te kiezen om later te worden gebruikt voor enting op NR moleculen: Hoofdstuk 6. Van de vier silaan types geeft VTES over het geheel genomen de slechtste eigenschappen vanwege z'n geringe afschermings-effect, en de vinyl-groep die moeilijk te koppelen is met NR rubber ketens in aanwezigheid van zwavel. Dientengevolge is VTES geen effectieve silaancoupling agent voor dit silica-gevulde NR systeem. Gebruik van NXT en VP Si-363 vertoont een goede verbetering in mengsel-eigenschappen, t.w. meng-energie, Mooney viscositeit, vulcanisatie-gedrag, bound rubber gehaltes, en een toename van mechanische en dynamisch mechanische eigenschappen vergeleken met helemaal geen silaan, maar de eigenschappen zijn inferieur t.o.v. gebruik van TESPT, voornamelijk vanwege lagere crosslink-dichtheden vergeleken met TESPT, dat een intrinsiek "zwaveldonerend" effect vertoont. Dit is verder niet nader onderzocht in de context van dit 
hoofdstuk. De incorporatie van NXT-silaan geeft betere scorch-safety, en toepassing bij een gelijke hoeveelheid ethoxy-functionele groepen t.o.v. TESPT geeft vulcanizaten met hetzelfde niveau treksterkte. Met betrekking tot banden-gedrag biedt NXT betere eigenschappen dan VP Si-363, maar nog wel iets inferieur t.o.v. het referentie-mengsel met TESPT.

Verderop worden NXT een VP Si-363 silaancouplingagents gebruikt om te enten op NR, teneinde reactieve zij-groepen in te brengen voor interactie en/of reactie met de silanol-groepen op het silica oppervlak. Hoofdstuk 7 laat zien dat de silaan-enting van de natuur rubbers succesvol verloopt onder condities van smelt-mengenin een interne menger bij $140{ }^{\circ} \mathrm{C}$, onder gebruikmaking van $0,1 \mathrm{phr} 1,1^{\prime}$-di(tert-butylperoxy)-3,3,5trimethylcyclohexaan of Luperox $\circledast 231$ XL40als initiator. Op basis van element-analyse via de SEM-EDX techniek bevatten de gemodificeerde NRs silicium ( $\mathrm{Si}$ ) atomen in hun structuren, en de hoeveelheden zuurstof en silicium in de geënte NRs nemen toe met hogere hoeveelheden silaan gebruikt voor de ent-reactie. Zowel VP Si-363-geënte NR als NXT-geënte NR laten infrarood absorptie-banden zien bij 3270, 1075 en $1035 \mathrm{~cm}^{-1}$, toegeschreven aan respectievelijkde deformaties van de $-\mathrm{OH}$, Si-O-C en Si-O-Si groepen, en de ${ }^{1} \mathrm{H}$ NMR absorptie band bij $3,8 \mathrm{ppm}$, behorende bij het methyleen-proton van de ethoxy-groepen.

Het gebruik van silaan-geënte NRs als compatibilizers in silica-gevulde mengsels wordt besproken in Hoofdstuk $\mathbf{8}$ in vergelijking met het regelrechte gebruik van silaan in de mengsel-formulering en gebruik van de optimale hoeveelheid TESPT. Toenemende hoeveelheden van NXT- en VP Si-363-geënte NRs als compatibilizers in silica-gevulde NR mengsels verlaagt de Mooney viscositeit, de vulstof-vulstof interactie of Payne effect, de snelheidsconstante voor flocculatie en verhoogt de chemische bound rubber gehaltes, $300 \%$ modulus, versterkings index en treksterkte. De versterkingsindex en treksterkte van de silica-gevulde NR vulcanizaten bereiken nagenoeg een plateau wanneer het silaangehalte in de silaan-geënte NRs, met inbegrip van vrije en geënte silaan, meer bedraagt dan 3,0 gew\% betrokken op de silica. Breukvlakken onder rek van de silica-gevulde NR vulcanizaten laten ductiele breuken zien met oppervlakte ruwheid en vele scheurlijnenwanneer de silaan-geënte NRs worden gebruikt, zoals in het geval van het mengsel met TESPT.Bij dezelfde silaan hoeveelheid geeft gebruik van silaan-geënte NR als compatibilizer een hogere verbetering in Payne effect, chemischbound rubber gehalte, $300 \%$ modulus en treksterkte in vergelijking met betere regelrecht gebruik van dezesilaan. De aanwezigheid van NXT geeft een hogere verbetering van de overall eigenschappen dan VP Si-363, maar beide liggen toch nog wel lager dan bij gebruik van de optimale hoeveelheid TESPT. In aanwezigheid van silaan-geënte NR als compatibilizerverschuift 
de $T_{g}$ van de silica-gevulde NR vulcanizaten naar een hogere temperatuur. De tan $\delta$ bij 5 ${ }^{\circ} \mathrm{C}$, welke relateert aan de natte grip, neemt iets af, maar de tan $\delta$ bij $60{ }^{\circ} \mathrm{C}$ wordt duidelijk verlaagd, hetgeen duidt op een lagere banden rolweerstand vergeleken met het referentie-mengsel met TESPT. Zwavel compensatie voor demengsels met NXT-geënte $\mathrm{NR}$ als compatibilizer wordt ook onderzocht om de eigenschappen van silica-gevulde NR te verbeteren, zoals besproken in Hoofdstuk 9. De zwavel-gecompenseerde mengsels vertonen een iets lagereMooney viscositeit, maar significant hoger vulcanisatiekoppel vergeleken met het mengsel zonder extra zwavel. Met zwavel-compensatie t.o.v. het zwavel-gehalte in het referentie TESPT mengsel, geeft het gebruik van NR-g-NXT met 6 gew\% NXT t.o.v. de silica een verlaging van de Mooney viscositeit en het Payne effect, evenals een verhoging van de $300 \%$ modulus en treksterkte van silica-gevulde NR tot nagenoeg hetzelfde niveau als bereikt met regelrecht gebruik van de optimale hoeveelheid TESPT, maar zonder significante effecten op de tan $\delta$ waardes bij 5 en 60 ${ }^{\circ} \mathrm{C}$. Aangezien de hoeveelheden van 8,6 gew\% TESPT en 6 gew\% NXT betrokken op de silica min of meer gelijke molaire hoeveelheden vertegenwoordigen, geeft het gebruik van NXT-geënte NR derhalve slechts de halve hoeveelheid ethanol-emissie t.o.v. van TESPT: milieu-technisch een vriendelijker en veiliger systeem.

Het toenemende belang van silica-versterkte banden mengsels ten behoeve van verlaging van het brandstof-verbruik van automobielen motiveert research en ontwikkeling van de relevante rubber technologie. Naast de gewoonlijk gebruikte silica/silaan technologie zijn alternatieve compatibilizers van belang om gedeeltelijk of volledig de silaan te vervangen door "groenere" producten. Het huidige proefschrift presenteert een diepgaande studie inde toepassing van ENRs als alternatieve compatibilizer. Gebruik van ENRs alleen als compatibilizers laat reeds een verbetering zien van de silica-NR compatibiliteit, de silica-dispersie en enige mechanische eigenschappen. Deze eigenschappen kunnen verder worden verbeterd door het gebruik van ENR te combineren met slechts kleine hoeveelheden silaancouplingagents en zwavel compensatie t.o.v. een referentie mengsel met TESPT. Naast het meer algemene gebruik van ENRs biedt silaangeënte NR een ander alternatief. De bereiding van silaan-geënte NR in de gesmolten meng-toestand is uitvoerbaar maar gecompliceerd. Het niet-gezuiverde product bevat zowel vrije als geënte silaan. Desalniettemin geeft het gebruik van niet-gezuiverde silaangeënte NR als compatibilizers een verbetering in vulstof-rubber interacties en de eigenschappen van silica-versterkte NR. Bij dezelfde totale hoeveelheid silaan geeft gebruik van silaan-geënte NR over het geheel genomen betere eigenschappen dan het regelrechte gebruik van silaan, en de eigenschappen kunnen nog verder verbeterd worden door zwavel compensatie, gebruikmakend van het mengsel metoptimale 
hoeveelheid TESPT als referentie. Over het geheel genomen, zelfs ofschoon de toepassing van chemisch gemodificeerde NRs in de vormen van ENRs en silaan-geënte NR als compatibilizers zonder verdere silaan resulteert in mindere eigenschappen vergeleken met het gebruik van state-of-the-art TESPT, biedt de juiste combinatie van gemodificeerde NR samen met slechts geringe hoeveelheden TESPT en zwavel compensatie een alternatief systeem met als voordelen gebruik van een op NR gebaseerd product en minder ethanol-emissie afkomstig van de silanizerings-reactie. Bovendien levert het gebruik van ENR een goede natte wet grip in het perspectief van de "Magische Driehoek van de Banden Technologie". 


\section{Symbols and Abbreviations}

\section{Symbol}

$\gamma$

$\gamma_{0}$

$\gamma_{s}$

$\gamma_{S}^{d}$

$\gamma_{s}^{s p}$

$\sigma$

$\sigma_{0}$

$\delta$

$\phi$

A

$\mathrm{E}_{0}$

E

$E_{A}$

$E^{\prime}$

$E^{\prime \prime}$

$\mathrm{G}^{\prime}$

G"

$\mathrm{H}$

I

$\mathrm{k}_{\mathrm{a}}$

M100

M300

M300/M100

$\mathrm{ML} 1+4\left(100^{\circ} \mathrm{C}\right)$

$\mathrm{R}$

T

$t_{c 90}$

$\mathrm{T}_{\mathrm{g}}$

$\mathrm{t}$

\section{Description}

Strain

Maximum amplitudes of strain

Surface free energy of fillers

Surface free energy of dispersive component

Surface free energy of specific component

Stress

Maximum amplitudes of stress

Phase angle between stress and strain

Volume fraction of filler

Arrhenius factor

Young's modulus of unfilled rubber

Young's modulus of filled rubber

Activation energy

Tension storage modulus

Tension loss modulus

Shear storage modulus

Shear loss modulus

Filler-filler networking factor

Polymer-filler interaction parameter

Flocculation rate constant

Tensile modulus at $100 \%$ strain

Tensile modulus at $300 \%$ strain

Reinforcement index

Mooney viscosity at 4 min Of test time, after preheating for 1

min measured at $100^{\circ} \mathrm{C}$ using a large rotor

Gas constant

Temperature

Optimum cure time

Glass transition temperatures

Time 
$t_{s 1}$

$\mathrm{x}$

Scorch time

Degree of flocculation

\section{Description}

AIBN

Azobisisobutylonitrile

ASTM

ATR-FTIR

American Society of Testing Materials

Attenuated Total Reflection-Fourier Transform Infrared

spectrometer

BET

Brunauer-Emmett-Teller

BR

Polybutadiene rubber

BTOS

i-Butyltriethoxysilane

CBS

$\mathrm{N}$-cyclohexyl benzothiazole sulfenamide

$\mathrm{CDCl}_{3}$

$\mathrm{CR}$

CSDPF

CTAB

CV

DBP

DCP

DMA

DMOD

DPG

DSC

ENR

ENR-xx

EpBR

EPDM

EPDM- $g$-VOS

EPM

EpSBR

ESCA

ETU

FT-IR

${ }^{1} \mathrm{H}-\mathrm{NMR}$

HA-NR

Deuterated chloroform

Polychloroprene rubber

Carbon-silica dual phase filler

Cetyltrimethyl ammonium bromide

Conventional vulcanization

Dibutylphthalate

Dicumyl peroxide

Dynamic mechanical analysis

$\mathrm{N}, \mathrm{N}$-dimethyl-1-octadecylamine

Diphenylguanidine

Differential scanning calorimetry

Epoxidized natural rubber

Epoxidized natural rubber with $x x$ mol\% epoxide content

Epoxidized polybutadiene

Ethylene-propylene-diene rubber

Vinyloxyaminosilane grafted ethylene-propylene-diene rubber

Ethylene propylene rubber

Epoxidized styrene-butadiene rubber

Electron spectroscopy for chemical analysis

Ethylene thiourea

Fourier-transform infrared spectroscopy

Proton nuclear magnetic resonance spectroscopy

High ammonia natural rubber latex 


$\begin{array}{ll}\text { HV-BR } & \text { High-vinyl polybutadiene rubber } \\ \text { IGC } & \text { Inverse gas chromatography } \\ \text { IIR } & \text { Isobutylene isoprene rubber } \\ \text { KPS } & \text { Potassium persulfate } \\ \text { LLDPE } & \text { Linear low density polyethylene } \\ \text { MFA } & \text { Multifunctional additive } \\ \text { MNR } & \text { Maleated natural rubber } \\ \text { MPS } & \text { Mercaptopropyl-trimethoxysilane } \\ \text { NBR } & \text { Acrylonitrile butadiene rubber } \\ \text { NR } & \text { Natural rubber } \\ \text { NR-g-NXT } & \text { Natural rubber grafted with NXT } \\ \text { NR-g-VP Si-363 } & \text { Natural rubber grafted with VP Si-363 } \\ \text { NRL } & \text { Natural rubber latex } \\ \text { NSA } & \text { Nitrogen surface area } \\ \text { NXT } & \text { 3-Octanoyl-thio-propyltriethoxysilane } \\ \text { OTES } & \text { N-octyltriethoxysilane } \\ \text { PDDA } & \text { Poly(diallyldimethylammonium chloride) } \\ \text { PE } & \text { Polyethylene } \\ \text { PP } & \text { Polypropylene } \\ \text { PP-g-MA } & \text { Poly(propylene-g-maleic anhydride) copolymer } \\ \text { RPA } & \text { Rubber process analyzer } \\ \text { RSS } & \text { Ribbed smoked sheet } \\ \text { SBR } & \text { Styrene-butadiene rubber } \\ \text { SEM } & \text { Scanning electron microscopy } \\ \text { SEM-EDX } & \text { Scanning Electron Microscopy - Energy Dispersive X-Ray } \\ \text { Semi-EV } & \text { Spectroscopy } \\ \text { SMO } & \text { Semi-efficient vulcanization } \\ \text { STSA } & \text { Sorbitan monooleate } \\ \text { TBBS } & \text { Statistical thickness surface area } \\ \text { TDAE } & \text { N-tert-butyl-2-benzothiazole sulfenamide } \\ \text { TEOS } & \text { Treated distillate aromatic extract oil } \\ \text { TESPT } & \text { Tetraethoxysilane } \\ \text { TESPD } & \\ \text { TMQ } & \text { BOS }\end{array}$


VP Si-363

VTES

VTMS

VTOS

$\mathrm{ZnO}$
3-Mercaptopropyl-di(tridecan-1-oxy-13-

penta(ethyleneoxide))ethoxysilane

Vinyltriethoxysilane

Vinyltrimethoxysilane

Vinylethoxysilane

Zinc oxide 


\section{Bibliography}

\section{Journal Articles}

1. K. Sengloyluan, K. Sahakaro, W.K. Dierkes and J.W.M. Noordermeer, Silicareinforced tire tread compounds compatibilized by using epoxidized natural rubber, European Polymer Journal, 51, 69-79 (2014).

2. K. Sengloyluan, K. Sahakaro and J.W.M. Noordermeer, Silica-reinforced natural rubber compounds compatibilized through the use of epoxide functional groups and TESPT combination, Advanced Materials Research, 844, 272-275 (2014).

3. K. Sengloyluan, K. Sahakaro, W.K. Dierkes and J.W.M. Noordermeer, Enhanced properties by a combination of epoxidized natural rubber and silane coupling agent of silica-reinforced natural rubber-based tire treads, European Polymer Journal, submitted.

4. K. Sengloyluan, K. Sahakaro, W.K. Dierkes and J.W.M. Noordermeer, Silicareinforced natural rubber compounds compatibilized by ENR in combination with different silane coupling agent types, Kautschuk Gummi Kunststoffe, in prepration.

5. K. Sengloyluan, K. Sahakaro, W.K. Dierkes and J.W.M. Noordermeer, Comparative reinforcement efficiency of silica in combination with different silane coupling agents in natural rubber-based tire compounds, Rubber Chemistry and Technology, in preparation.

6. K. Sengloyluan, K. Sahakaro, W.K. Dierkes and J.W.M. Noordermeer, Functionalized natural rubber by grafting reaction with silanes, Express Polymer Letters, in preparation.

7. K. Sengloyluan, K. Sahakaro, W.K. Dierkes and J.W.M. Noordermeer, Compatibilization of silica-filled natural rubber by using silane-grafted-NR, Rubber Chemistry and Technology, in preparation.

\section{Presentations}

1. K. Sengloyluan, K. Sahakaro, W. K. Dierkes and J. W.M. Noordermeer, Effect of epoxidized natural rubber used as a compatibilizer in silica-filled natural rubber, Dutch Polymer Days - 2012, Lunteren, the Netherlands, March 12-13, 2012. 
2. K. Sengloyluan, K. Sahakaro and J.W.M. Noordermeer, Use of epoxidized natural rubber as a compatibilizer in silica-filled natural rubber tire tread compounds, Deutsche Kautschuk-Tagung (DKT) 2012, Nuremberg, Germany, July 2-5, 2012.

3. K. Sengloyluan, K. Sahakaro and J.W.M. Noordermeer, Use of epoxidized natural rubber as a compatibilizer in silica-filled natural rubber compounds, The $28^{\text {th }}$ International Conference of Polymer Processing Society (PPS-28), Pattaya, Thailand, December 11-15, 2012.

4. K. Sengloyluan, K. Sahakaro and J.W.M. Noordermeer, Use of epoxidized natural rubber as a compatibilizer in silica-filled natural rubber compounds, The $3^{\text {rd }}$ Sci-tech Grad Symposium, Prince of Songkla University, Pattani, Thailand, March 20, 2013.

5. K. Sengloyluan, K. Sahakaro and J.W.M. Noordermeer, Silica-reinforced natural rubber compounds compatibilized through the use of epoxide functional groups and TESPT combination, The $1^{\text {st }}$ Asia Pacific Rubber Conference (APRC) 2013, Suratthani, Thailand, September 5-6, 2013.

6. K. Sengloyluan, K. Sahakaro, W.K. Dierkes and J.W.M. Noordermeer, Effect of TESPT and extra sulfur on the properties of silica-reinforced natural rubber with epoxidized natural rubber as compatibilizer, The $4^{\text {th }}$ Polymer Conference of Thailand (PCT-4), Bangkok, Thailand, March 20-21, 2014. 


\section{Acknowledgement}

I started my role as a Ph.D. student in September 2010 under a double degree Ph.D. program between University of Twente in the Netherlands and Prince of Songkla University, Pattani campus in Thailand with the financial supported from the Netherlands Natural Rubber Foundation. During the past 4 years, I studied and worked for 2.5 years at Department of Rubber Technology and Polymer Science (Rubber Tech.), Prince of Songkla University (PSU), Thailand and for 1.5 years at Department of Elastomer Technology and Engineering (ETE), University of Twente (UT), the Netherlands. From the started, I have got lots of help and support from many people. I would like to thank and express my sincere gratitude to all of them and institutions involved in this co-operation program.

I may have not been succeeded as a Ph.D. without Prof. Dr. J.W.M. Noordermeer and Dr. Kannika Sahakaro, who gave me a good opportunity to join in this co-operation program. I would like to appreciate and express my deep sense of gratitude to both of them for teaching, coaching and helping me throughout this period. I have always appreciated in your high standard and quality of work. I have gained lots of experiences, not only about academic knowledge but also work and lifestyle especially when I was abroad. It is the most precious time for me to work under your supervision. Thank you very much for being the promotors for my thesis. Dr. Kannika, I know you have a hard time to train me to prepare and write articles and chapters in the proper way. Even I discouraged to reach my point sometime, you always encouraged me with your active suggestions and constant support, thank you very much.

I would like to appreciate and express my respect to Prof. Dr. A.Blume and Dr. W.K. Dierkes for your help and support when I was a part of ETE group. Thank you very much for investing your valuable time for suggesting and correcting my articles and chapters. Even though we had only a few times for discussion, but every time I learned a lot of things from your knowledge and experiences that made my thesis possible.

It is my pleasure to express my sincerely thank to all of staff from Rubber Tech. and ETE groups for training, teaching and supporting me. Dear P-Somkid, P-Siradanai, PApichart, P-Inyas P-Absorn and Vidchapon, thank you very much for your co-operation and everything that you have done for me. Dear Jacob, you are absolutely incredible person. I'm glad to have opportunity to know you. I always appreciate and remember what 
you have done, including a correction of my English skill. Dear Yvonne and Ceciel, thank you for helping and taking care of me.

I would like to appreciate and express my regards to Prof. Dr. G.P.M.R. Dewulf, Prof. Dr. Ir. D.J. Schipper, Dr. Ir. P.J. Dijkstra, Prof. Dr. U. Giese, Prof. Dr. J. Vourinen, Dr. Ir. L.A.E.M. Reuvekamp and Dr. A.V. Chapman for being my graduation committee.

I would like to convey my thankful to all of the former Ph.D. researchers in this field. I learned a lot of things through all sentences in their theses. What they have done before is really useful for my work.

Dear Dr. Sitisyidah Saiwaree and family, thank you very much for teaching, supporting and taking care of me when I was in the Netherlands for the first time. PPrachid, P-Anee, P-Methakarn, P-Wisut and P-Ekwipoo, thank you very much for supporting and encouraging me to pass a hard time during my education period. You are a good example for me in both studying and working.

To all of my colleagues in ETE group; Salina, Ernest, Morteza, Somayeh, Andre, Nadia, Gerda, Vignesh Kumar, Akansha, Monika, Katazyna and Indria, I had a good time when I spent my time in the group with all of you. This is my first time to study and live oversea, it is a challenge and adventure in the same time. Thank you very much for your sincerely friendship and cheerful that you all giving me all the time. I appreciated to all of activities that we had done together.

Thank you very much to Thai people in Enschede; P-May, P-Tom, P-Waew, PPipe, P-Rex, P-Neung, Si, Gift, N-Meaw, N-Ham, N-May, N-Golf, N-Wa, you made my days in oversea more colorful. Spending sometimes together for cooking and traveling made me feel like we were family and helped me ease my loneliness. My special thanks to André and Vignesh Kumar for being my paranymfen.

My sincere thanks to my colleagues in Thailand; P-Tuk, P-Pui, P-Ball, P-Alif, PDee, Ang, Chalao, Hakim, Suradech, N-Dream, N-Peung and others who have become my good friends. Special thank to my lovely and close friends; Nah and Da, we have shared our happiness and sadness together. We have chosen to walk in the same fate, I wish we will reach the goal together.

My utmost thanks go to my beloved family; my father: Nauy, my mother: Pin, my sisters: Keaw and Koi and also my beloved relatives. Thank you for giving me your unconditional love, being by my side and supporting me.

Karnda Sengloyluan

June, 2015 


\section{Curriculum Vitae}

Karnda Sengloylan was born on November $29^{\text {th }}, 1985$, in Satun, Thailand. She received her B.Sc. degree in Rubber Technology in 2008 and Master degree in Polymer Technology in 2010, from Prince of Songkla University, Pattani Campus, Thailand. She finished her bachelor project under the topic of "Mechanical and dynamic mechanical properties of NR/EPDM blends". After she got her bachelor degree, she continued to study her master in Polymer Technology program. The research title of her master project was "Effect of silica and clay on rheological, mechanical and thermal properties of thermoplastic vulcanizates based on epoxidized natural rubber and polypropylene blends". In September 2010, she started her Ph.D. under the supervision of Prof. Dr. J.W.M. Noordermeer and Dr. Kannika Sahakaro in the co-operation program of University of Twente in the Netherlands and Prince of Songkla University in Thailand. Her project was focused on "Silica-reinforced natural rubber: use of natural rubber grafted with chemical functionalities as compatibilizer". This research was sponsored by the Netherlands Natural Rubber Foundation. 
"You can fool all the people some of the time, and some of the people all the time, but you cannot fool all the people all the time." 